\title{
Aggregation and pattern formation in charged granular gases
}

\author{
Dissertation \\ for the award of the degree \\ "Doctor rerum naturalium" \\ of the Georg-August-Universität Göttingen
}

within the doctoral program

Physics of Biological and Complex Systems

of the Georg-August University School of Science (GAUSS)

\author{
submitted by \\ Chamkor Singh \\ from Hanuman Garh, India
}

Göttingen 2019 


\section{Thesis committee}

Prof. Dr. Marco G. Mazza

Research Group: Non-Equilibrium Soft Matter

Max Planck Institute for Dynamics and Self Organization, Göttingen

Prof. Dr. Eberhard Bodenschatz

Department of Fluid Physics, Pattern Formation and Biocomplexity Max Planck Institute for Dynamics and Self Organization, Göttingen

Prof. Dr. Reiner Kree

Institute for Theoretical Physics, University of Göttingen

\section{Thesis referees}

Prof. Dr. Marco G. Mazza

Research Group: Non-Equilibrium Soft Matter

Max Planck Institute for Dynamics and Self Organization, Göttingen

Prof. Dr. Eberhard Bodenschatz

Department of Fluid Physics, Pattern Formation and Biocomplexity

Max Planck Institute for Dynamics and Self Organization, Göttingen 


\section{Members of the examination board}

Prof. Dr. Marco G. Mazza

Research Group: Non-Equilibrium Soft Matter

Max Planck Institute for Dynamics and Self Organization, Göttingen

Prof. Dr. Eberhard Bodenschatz

Department of Fluid Physics, Pattern Formation and Biocomplexity

Max Planck Institute for Dynamics and Self Organization, Göttingen

Prof. Dr. Reiner Kree

Institute for Theoretical Physics, University of Göttingen

Prof. Dr. Matthias Krüger

Institute for Theoretical Physics, University of Göttingen

Dr. Claus Heussinger

Institute for Theoretical Physics, University of Göttingen

Prof. Dr. Stefan Klumpp

Institute for Dynamics of Complex Systems, University of Göttingen

\section{Date of oral examination}

02.09.2019 

to Basant and Gurmeet. 



\section{Publications from the thesis}

C. Singh and M.G. Mazza, Electrification in granular gases leads to constrained fractal growth, Scientific Reports 9: 9049 (2019).

C. Singh and M.G. Mazza, Early-stage aggregation in three-dimensional charged granular gas, Physical Review E 97: 022904 (2018).

\section{Invited talk from the thesis}

Particle aggregation in 3-D charged granular gas: simulations and mean-field study, Gordon Research Seminar - Granular Matter (Frontiers of Granular Physics Session), Easton, Massachusetts, USA, July 2018. 



\section{Contents}

$\begin{array}{ll}\text { Abstract } & \text { xiii }\end{array}$

$\begin{array}{ll}\text { Acknowledgements } & \text { xv }\end{array}$

$\begin{array}{ll}\text { List of Symbols } & \text { xxi }\end{array}$

$\begin{array}{ll}\text { List of Abbreviations } & \text { xxiii }\end{array}$

$\begin{array}{ll}\text { List of Figures } & \text { xxxii }\end{array}$

$\begin{array}{ll}\text { List of Tables } & \text { xxxiii }\end{array}$

1 Introduction 1

1.1 Motivation . . . . . . . . . . . . . . . . . . . 1

1.2 Literature review . . . . . . . . . . . . . . . 4

1.2.1 Granular frictional or tribo, and contact electrification . . . . . 5

1.2.2 Charged suspensions, powders, colloids, and dusty plasma . . . 6

1.2.3 Aggregation of charged particles and astrophysical applications . 7

1.2.4 Collective effects in flows of charged particles . . . . . . . . . 8

1.2.5 Charged granular gases and their scope . . . . . . . . . . 9

1.3 Extent, scope and organization of the thesis . . . . . . . . . . 10

2 Theoretical framework $\quad 13$

2.1 Statistical distribution function . . . . . . . . . . . . . . 13

2.2 Boltzmann equation for a charged granular gas . . . . . . . . . . . . . . . 14

2.3 Macroscopic quantities . . . . . . . . . . . . . . . . . 15

2.4 Collision integral . . . . . . . . . . . . . . . . 16

2.4.1 Collision integral for a neutral granular gas . . . . . . . . 20

2.4.2 Modified collision integral for a charged granular gas . . . . . . 22

2.5 Mean-field description of aggregation: Smoluchowski coagulation equation 24

3 Granular molecular dynamics $\quad 27$

3.1 Equation of motion . . . . . . . . . . . . . . . . . 27

3.2 Contact forces . . . . . . . . . . . . . . . 28

3.3 Long-range forces: Ewald summation . . . . . . . . . . . . . 31

3.4 Long-range forces: Induced polarization . . . . . . . . . . . . . . . 34

3.5 Charge currents . . . . . . . . . . . . . . . 36

3.6 Rescaling of the equations: Non-dimensional numbers . . . . . . . . . 36 
3.7 Numerical integration of the equations of motion $\ldots \ldots \ldots \ldots$

3.7 .1 Gear's integration scheme . . . . . . . . . . . . . . . 40

3.7.2 Velocity Verlet integration scheme . . . . . . . . . . . 41

3.8 Integration of the stochastic differential equation for charge $\ldots \ldots . .42$

3.9 Non physicality of random walk charge-exchange . . . . . . . . . . 44

3.10 Charge-exchange with multiplicative noise . . . . . . . . . . 50

3.11 Viscous friction: Preliminary . . . . . . . . . . . . . 50

3.12 Software and hardware used $\ldots \ldots \ldots \ldots$

4 Early-stage aggregation in three-dimensional charged granular gas $\mathbf{5 3}$

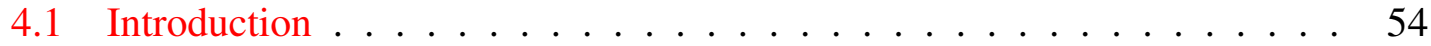

4.2 Model . . . . . . . . . . . . . . . . . . . . 56

4.3 Initialization . . . . . . . . . . . . . . . 62

4.4 Results and discussion $\ldots \ldots \ldots \ldots \ldots \ldots \ldots \ldots$

4.4 .1 Clustering . . . . . . . . . . . . . . . 66

4.4.2 Mean-field: Smoluchowski aggregation equation with modified reaction kernel . . . . . . . . . . . . . . . . . . . 68

4.4 .3 Velocity distribution . . . . . . . . . . . . . . . 73

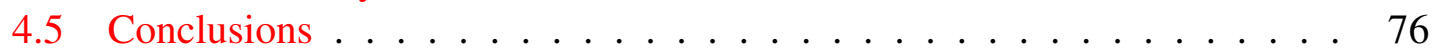

5 Electrification in charged granular gases leads to constrained fractal growth $\mathbf{7 9}$

5.1 Introduction . . . . . . . . . . . . . . . . . 81

5.2 Kinetics . . . . . . . . . . . . . . . . . . 82

5.3 Results . . . . . . . . . . . . . . . . . . . . . . 89

5.3.1 Inhomogeneous aggregation and fractal growth . . . . . . . 92

5.3.2 Interplay between fractals and mesoscopic flow . . . . . . . . 94

5.4 Methods . . . . . . . . . . . . . . . . . . 95

5.4.1 Kinetics and modified collision integral . . . . . . . . . . 95

5.4.2 Splitting restitution and aggregation . . . . . . . . . 97

5.4.3 Derivation of the hydrodynamic equations (5.6)-(5.8) . . . . . . 101

5.4.4 Solution for the restitutive part $\left(\frac{3}{2} \frac{\partial T}{\partial t}\right)_{\text {res }} \ldots \ldots \ldots \ldots \ldots$

5.4.5 Solution for the aggregative part $\left(\frac{3}{2} \frac{\partial T}{\partial t}\right)_{\text {agg }} \ldots \ldots \ldots \ldots \ldots$

5.4.6 Granular MD simulations . . . . . . . . . . . . . . 106

5.5 Comparison of individual $T,\left\langle\delta q^{2}\right\rangle$, and $n$ profiles, and emergence of convective flow using Mach number . . . . . . . . . . . . . . . 107

5.6 Reference scales and laboratory relevance of present results . . . . . . 110

5.7 Conclusions and discussion . . . . . . . . . . . . . . . . . 111

6 There is more happening before aggregation 115

6.1 Dimensional analysis . . . . . . . . . . . . . . . . . . 116

6.2 Hydrodynamics in the limit $\frac{k_{e} q_{\text {ref }}^{2}}{\bar{r}} \ll T\left|\epsilon^{2}-1\right| \ldots \ldots \ldots . . \ldots 117$

6.2 .1 Mass transport . . . . . . . . . . . . . . . . . . 118

6.2 .2 Momentum transport . . . . . . . . . . . . . . . . . . . . . 119

6.2 .3 Kinetic energy transport . . . . . . . . . . . . . . . 120

6.2 .4 Charge transport . . . . . . . . . . . . . . . . . 120

6.2.5 Pressure tensor, heat flux, and transport coefficients . . . . . . . 122

6.3 Linear stability of the hydrodynamic equations . . . . . . . . 122 
6.3.1 Transverse modes . . . . . . . . . . . . . . . . 126

6.3.2 Longitudinal/parallel and other hydrodynamic modes . . . . . . . 126

7 Effect of induced polarization and viscous friction: Preliminary 131

7.1 Polarized, heterogeneously charged granular gas . . . . . . . . . . . . . 132

7.1.1 Cluster mass/size versus cluster dipole moment . . . . . . . . . . 132

7.1.2 Effect on the fractal dimension . . . . . . . . . . . . . . . 134

7.1.3 Effect on the average cluster size . . . . . . . . . . . 136

7.2 Effect of drag due to random interstitial velocities . . . . . . . . . . . 137

7.3 Conclusions . . . . . . . . . . . . . . . . 138

8 Conclusions, Outlook, and Extensions 143

8.1 Summary of conclusions . . . . . . . . . . . . . . . . . 144

8.2 Outlook and possible extensions . . . . . . . . . . . . . . . 147

Appendix 1: Accompanying media and source codes 149

Appendix 2: Overall granular molecular dynamics algorithm 151

Appendix 3: MATLAB Scripts - Eigenvalues of the linear stability matrix $\quad 153$

Appendix 4: MATHEMATICA Scripts - Dipole interactions and kinetic integrals 155

Appendix 5: Highly nonlinear regions on macroscopic scale 159

$\begin{array}{ll}\text { References } & 161\end{array}$

$\begin{array}{ll}\text { Curriculum Vitae } & 169\end{array}$ 



\section{Abstract}

Particulate flows of dielectric grains are almost always plagued by contact or triboelectrification and the resulting long-range electrostatic interactions. Extensive experimental, but rare theoretical, work is ongoing at present to understand the underlying mechanisms of contact electrification, and its consequences on collective scales. Although the hard matter principles remain elusive, here an effort is made to theoretically understand the collective/many-body effects in granular gases arising due to this phenomena.

This thesis is focused on understanding the aggregation and pattern formation in heterogeneously charged, globally charge conserving, and initially dilute granular gases; the gas is charged with simplified but physically valid charge-exchange recipes and the subsequent effects of the long-range forces on the dynamics and morphology are studied. Canonical observables, such as growth rates of the average cluster size, average fractal dimension, granular temperature, and charge variance are computed using granular molecular dynamics simulations. In addition, the size, velocity and charge distributions are also studied. The observations from the detailed numerical simulations are utilized to (i) modify the mean field Smoluchowski's coagulation equation, and (ii) to provide a kinetic description taking care of restitution as well as aggregation. The onset of clustering instability and the competition between dissipation and electrostatics, on a macroscopic scale, is then studied via the linear stability analysis of the granular hydrodynamic equations. Finally, the alterations in the aggregate growth rates and the fractal dimension due to additional degrees of freedom, introduced by the grain polarizability, are briefed.

Most important results, in general terms, from the thesis are as follows. The presence of electrostatics fundamentally changes the nature of clustering in a granular gas - from dynamic clustering to actual aggregation/coagulation. The growth rates of the clusters are enhanced. The morphology of the emerging structures is self-similar and exhibits fractal nature at simulated length and time scales. However, it is found that the heterogeneous charges and aggregation have a negligible influence on the established statistical result of granular temperature decay - the Haff's law. The presence of charges fetches the granular gas further far-from equilibrium relative to its neutral counterpart, suggested by a nonrelaxing velocity distribution and some evidence of the charge-velocity correlations. In case of large scale charge separation in the gas, the spatial regions can be differentiated to $(i)$ where the electrostatic origin of the onset of clustering instability is expected to be linear, and (ii) where it is in principle nonlinear. The grain polarizability affects the small sized aggregate population; the fractal dimension of this subpopulation is lowered. The overall cluster growth is further enhanced due to the polarizability. Finally, it is demonstrated that only small-sized aggregates are expected to survive if they are "hit" by random interstitial drag - a scenario applicable to astrophysical dust growth processes. 



\section{Acknowledgements}

First of all, I would like to thank my advisor Prof. Marco G. Mazza for his supervision and support. I would also like to sincerely thank my thesis committee members: Prof. Reiner Kree and Prof. Eberhard Bodenschatz for useful comments and suggestions, and also for critical feedback which encouraged me to think from different perspectives. A sincere thanks also goes to Prof. Jürgen Blum for insightful conversations on intricacies of dust-agglomeration processes. I thank the Max-Planck Society for funding. A special thanks goes to Thomas Eggers for IT support, and my colleague Jana Lasser for her help during tough times. I also thank my office mate Thomas Breithaupt for cheerful breaks. I would like to thank the GGNB coordinating staff. Last but not least, a heartfelt thanks goes to all my family, friends and respected teachers back from my country without whom I could not be here. 



\section{List of Symbols}

\section{Dimensionless numbers}

$\mathcal{A} \quad$ Non-dimensional polarizability

$\mathcal{B} \quad$ Bjerrum number

$\mathcal{C} \quad$ Non-dimensional charge holding capacity of a grain

$\mathcal{D} \quad$ Ratio of characteristic dissipative to characteristic inertial force

$\mathcal{E} \quad$ Ratio of characteristic elastic to characteristic inertial force

$\mathcal{K}$ Ratio of characteristic Coulomb to characteristic inertial force

$\mathcal{M}$ Mach number

$\mathcal{Q}$ Non-dimensional charge-exchange parameter/strength of charge-exchange currents

$\mathcal{S} \quad$ Stokes number

Kn Knudsen number

\section{Mathematical symbols}

$\alpha_{\mu} \quad$ Electric polarizability of grain material

$\overline{\mathbf{I}} \quad$ Identity matrix

$\overline{\mathbf{P}} \quad$ Kinetic part of the pressure tensor

$\bar{q} \quad$ Absolute mean charge

$\bar{r} \quad$ Mean free path of granular gas particles

$\bar{r}_{\text {gas }} \quad$ Mean free path of interstitial gas molecules

$\boldsymbol{\mu} \quad$ Dipole-moment of a grain

$\omega \quad$ Angular velocity of a grain

$\chi \quad$ Determinant of Jacobian of a transformation

$\Delta t \quad$ Numerical time step 
$\ell \quad$ Length

$\ell_{\mathrm{B}} \quad$ Bjerrum length

$\epsilon \quad$ Coefficient of restitution

$\eta \quad$ Shear viscosity in the granular hydrodynamic equations

$\gamma \quad$ Parameter related to particle's material viscosity

$\kappa \quad$ Thermal conductivity in the granular hydrodynamic equations

b Vector of integers $\left(b_{x}, b_{y}, b_{z}\right) \in \mathbb{Z}^{3}$

E Macroscopic or mean electric field

e Electric field at the location of a grain

$\mathbf{F}^{\text {long }}$ Net long-range force on a grain

$\mathbf{F}^{\text {short }}$ Net short-range force on a grain

$\mathbf{F}_{i j} \quad$ Net force on a grain $i$ exerted by grain $j$

$\mathbf{n}_{i j} \quad$ Unit vector pointing from the center of mass of grain $i$ towards the center of mass of grain $j$

Q Heat flux vector

$\mathbf{r}_{i} \quad$ Position vector of a grain $i$

$\mathbf{r}_{i j}=\mathbf{r}_{j}-\mathbf{r}_{i}$ Position of center of mass of grain $j$ relative to the center of mass of grain $i$

$\mathbf{T} \quad$ Net torque on a grain

V Macroscopic or mean velocity

$\mathbf{v} \quad$ Center of mass velocity of a grain

$\mathbf{v}_{\text {fluid }}$ Fluid velocity

$\mathbf{v}_{i j}=\mathbf{v}_{j}-\mathbf{v}_{i}$ Velocity of center of mass of grain $j$ relative to the center of mass of grain

$\mathcal{V} \quad$ Set of phase space variables other than $\mathbf{r}, t$

$\nu \quad$ Collision frequency

$\nu_{\mathrm{P}} \quad$ Poisson's ratio

$\Omega \quad$ Solid angle

$\phi \quad$ Filling fraction

$\phi_{\mathrm{hcp}} \quad$ Filling fraction corresponding to hexagonal close packing 
$\Psi \quad$ Stochastic noise

$\rho=n m_{0}$ Mass density

$\rho_{\mathrm{I}} \quad$ Electrical resistivity of particle material

$\rho_{\mathrm{m}} \quad$ Material mass density

$\rho_{\mathrm{q}} \quad$ Charge density, surface or volumetric

$\sigma \quad$ Total collision cross section

$\tau_{\text {fric }} \quad$ Friction time

$\Theta \quad$ Heaviside step function

$\varepsilon \quad$ Permittivity of a medium

$\varepsilon_{0} \quad$ Permittivity of vacuum

$\varepsilon_{\mathrm{r}} \quad$ Relative permittivity

$\xi_{i j} \quad$ Mutual compression between grain $i$ and $j$ during collision

$\zeta \quad$ Granular cooling coefficient

A Elastic constant for linear spring-dashpot model of collision between grains

$B \quad$ Dissipative constant for linear spring-dashpot model of collision between grains

b Impact parameter

$D \quad$ Dissipative constant for viscoelastic model of collision between grains

$d \quad$ Characteristic size of aggregate/cluster

$d_{0} \quad$ Grain/monomer diameter

$D_{f} \quad$ Fractal dimension

E Elastic constant for viscoelastic model of collision between grains

$e \quad$ Magnitude of electron charge

$E_{\text {kin }} \quad$ Kinetic energy

$f \quad$ Probability distribution function

G Gravitational constant

I Electric current

$I^{\text {coll }}$ Collision integral

$K \quad$ Used interchangeably for aggregation/reaction kernel, or inverse of the energy required to transfer one elementary charge between grains, as described in the text 
$k_{e} \quad$ Coulomb's constant

$L \quad$ Linear size of simulation box

$M \quad$ Moment of inertia of a grain about an axis through its center of mass

$m \quad$ Mass of an aggregate or cluster

$m^{\text {eff }} \quad$ Reduced mass of a colliding pair

$m_{0} \quad$ Mass of a grain

$n \quad$ Number density

$N, N_{\text {mon }}$ Number of monomers

$N_{\text {boxes }}$ Number of boxes

$N_{\text {stick }}$ Coordination number, or number of particles sticking to a given particle

$Q \quad$ Macroscopic or mean charge

$q \quad$ Electric charge on a grain

$R \quad$ Radius of a grains

$R^{\text {eff }} \quad$ Effective radius of a colliding pair of grains

$r_{\mathrm{c}} \quad$ Cutoff radius/distance

$R_{g} \quad$ Aggregate/cluster radius of gyration

$S \quad$ Average cluster size

$s \quad$ Sticking probability

$t \quad$ Time

$T, T_{\mathrm{g}}$ Granular temperature

$U, \varphi$ Electrostatic potential

$Y \quad$ Youngs modulus

$Z \quad$ Integer denoting number of elementary charges

\section{Subscripts and superscripts}
agg Aggregates
coll Collision
eff Effective or reduced
fric Friction 


$\begin{array}{ll}\text { g } & \text { Granular } \\ \text { long } & \text { Long-range } \\ \text { mon } & \text { Monomers } \\ \text { pol } & \text { Polarization } \\ \text { ref } & \text { Reference value } \\ \text { short } & \text { Short-range }\end{array}$

\section{Other notations used in the thesis}

$\overline{\mathbf{x}} \quad$ Representation of a tensor

$\overline{\mathbf{x}}: \overline{\mathbf{y}}$ Contraction of two tensors $\overline{\mathbf{x}}$ and $\overline{\mathbf{y}}$

$\delta x \quad$ Variation of a variable $x$

$\dot{x} \quad$ Time derivative of a variable $x$

$\equiv \quad$ By definition, or equivalent to

$\lesssim \quad$ Less than or approximately equal to

$\mathrm{x} \quad$ Representation of a vector

$\mathcal{O}(x)$ Of the order of $x$

$\sim \quad$ Used loosely to emphasize scales as, or of the order of

$\tilde{x}, x^{*} \quad$ Rescaled non-dimensional value of a variable $x$

$\mathbf{x y}, \mathbf{x} \otimes \mathbf{y}$ Tensor product of two vectors $\mathbf{x}$ and $\mathbf{y}$ 



\title{
List of Abbreviations
}

\author{
BA Ballistic aggregation. \\ BCCA Ballistic cluster-cluster aggregation. \\ CC Charge-charge interaction. \\ CCA Cluster-cluster aggregation. \\ CD Charge-dipole interaction. \\ COM Center of mass. \\ DC Dipole-charge interaction. \\ DD Dipole-dipole interaction. \\ DLA Diffusion limited aggregation. \\ DLPCA Diffusion limited particle-cluster aggregation. \\ ELA Eden like aggregation. \\ EOM Equation of motion. \\ HCS Homogeneous cooling state. \\ ICS Inhomogeneous cooling state. \\ LHS Left-hand side. \\ MD Molecular dynamics. \\ MSD Mean square displacement. \\ PCA Particle-cluster aggregation. \\ PDF Probability distribution function. \\ RHS Right-hand side. \\ RLA Reaction limited aggregation.
}





\section{List of Figures}

1.1 (a) The Pillars of Creation of Eagle's nebula observed by the Hubble space telescope, and (b) exaggerated view of a cosmic dust cluster in the nebula. Original image source: ESA/Hubble. weblink: https://www.spacetelescope.org/images/heic1501a. . . . . . . . . .

1.2 Typical examples from medicine, mining, agriculture and mineral industries. (a) Medicinal tablets, (b) ferric-oxide, (c) raw food grains, and (d) salt and pepper used in everyday life. . . . . . . . . . . . . . . . .

1.3 (a) Lightening due to static electrification of volcanic ash particles, and (b) an artist's impression of electrically charged dust strom on Mars. . . .

2.1 Geometrical description of a binary collision between neutral particles. A particle $j$ collides with particle $i$, within a differential time $d t$, only if the relative velocity $\mathbf{v}_{i j}$ points towards a region covered by the impact parameter $b$, and the particle $j$ is within a distance $\left|\mathbf{v}_{i j}\right| d t$ from the point of contact. The direction $\mathbf{n}_{i j}$ is the unit vector from the center of particle $i$ towards the center of particle $j$ at the instant of contact, and $\frac{d \sigma}{d \Omega}$ is the differential cross section per unit solid angle around $\mathbf{n}_{i j} \ldots \ldots \ldots$

3.1 The relative velocity just before the collision between two grains $i$ and $j$ is $\mathbf{v}_{i j}=\mathbf{v}_{j}-\mathbf{v}_{i}$, and just after the collision is $\mathbf{v}_{i j}^{\prime}=\mathbf{v}_{j}^{\prime}-\mathbf{v}_{i}^{\prime}$. The normal collision velocity is $\mathbf{v}_{n}=\left(\mathbf{v}_{i j} \cdot \mathbf{n}_{i j}\right) \mathbf{n}_{i j}$ where $\mathbf{n}_{i j}=\frac{\mathbf{r}_{j}-\mathbf{r}_{i}}{\left|\mathbf{r}_{j}-\mathbf{r}_{i}\right|}=$ $\frac{\mathbf{r}_{i j}}{\left|\mathbf{r}_{i j}\right|}$ is a unit vector pointing from the center of particle $i$ towards the center of particle $j$. The normal collision velocity just after the collision is related to the normal collision velocity just before the collision as $\mathbf{v}_{n}^{\prime}=$ $-\epsilon \mathbf{v}_{n}$ where $0 \leq \epsilon \leq 1$ is the coefficient of normal restitution. Similarly the tangential collision velocity just after the collision is related to the tangential collision velocity just before the collision as $\mathbf{v}_{t}^{\prime}=\epsilon_{t} \mathbf{v}_{t}$ where $\epsilon_{t}$ is the coefficient of tangential restitution. In the present study $\epsilon_{t}=1$. During the course of the collision, the mutual deformation of the particles is denoted as $\xi_{i j}(t)=d_{0}-\left|\mathbf{r}_{i j}(t)\right| \ldots \ldots \ldots \ldots$

3.2 A schematic representation of the treatment of long-range charge-charge interactions (CC), charge-dipole interactions (CD), dipole-charge interactions (DC), and dipole-dipole interactions (DD). While CC interactions are dealt with using the Ewald summation, the minimum image convention is used for the other three cases. . . . . . . . . . . . . . . . 
3.3 The charge exchange current $I_{j i}(t)$ during the course of collision between two grains $i$ and $j$. The time dependent contact area $A_{j i}(t)$ during the course of collision is shown on the right, indicated by section XX. The instantaneous area during collision can be related to the mutual particle compression $\xi_{i j}$ as: $A_{j i}(t)=\pi \xi_{i j}(t)\left[d_{0}-\xi_{i j}(t)\right]$. See also Eq. (3.5) and Fig. 3.1 for $\xi_{i j} \ldots \ldots \ldots \ldots$. . . . . . . . . . . . 36

3.4 Charging and sticking of glass particles in a drop tower experiments (Image from private communication with Prof. Gerhard Wurm, Universität Duisburg Essen). URL: https://www.unidue.de/imperia/md/images/physik/agw/sticking.gif. . . . . . . . . . .

3.5 (a) The average kinetic energy and the granular temperature of a granular gas with random walk charge-exchange currents. This mechanism of charge-exchange leads to energy injection into the system, which balances the dissipation at long times. (b) The average kinetic energy and the granular temperature of a granular gas with modified charge-exchange currents. This mechanism of charge-exchange reproduces Haff's law at long times. Here $v_{i}$ is the grain speed and $V$ is the mean advective velocity in the vicinity of grain $i \ldots \ldots \ldots \ldots \ldots$

3.6 Time evolution of the granular gas while taking $z=0.0$ in the initial charge distribution Eq. (3.49), with random-walk charge exchange model.

3.7 Time evolution of the granular gas while taking $z=2.0$ in the initial charge distribution Eq. (3.49), with random-walk charge exchange model.. Time evolution of the granular gas while taking $z=8.0$ in the initial charge distribution Eq. (3.49), with random-walk charge exchange model. For the above three values of $z$, the charge distribution converges to the same stationary shape. The granular temperature reaches a steady state, which implies that the simplistic random-walk/Langevin like charge exchange acts as an energy injection balancing the dissipation. This observation indicates that the charge exchange cannot simply be a random-walk process. Also spatially the gas remains homogeneous which is contradictory to experimental observations of aggregate formation. . . . . . . . . 
4.1 (a) The evolution of the granular temperature $T$ (or $T_{\mathrm{g}}$ ) for a purely repulsive dilute granular gas with monopolarly charged particles and constant coefficient of restitution $\epsilon$. We study the dependence on the ratio of Coulomb to thermal energy $\mathcal{K}$. The $\mathcal{K}=0$ curve corresponds to a neutral granular gas. At very short times, the granular gas follows Haff's (Haff, 1983) law ( $T(t) \sim t^{-2}$ ) in the homogeneous cooling state. The repulsive electrostatic interactions among the particles reduce the collision frequency and thus result in a slower decay of $T$ as time progresses $\left(T(t) \sim 1 / \ln \left(t / t_{c}\right)\right.$, also shown analytically by Scheffler \& Wolf (2002)). As $\mathcal{K}$ increases, the deviation from Haff's law is more pronounced and occurs earlier in time. The solid line represents the theoretical prediction of Haff's law for a neutral granular gas with $\epsilon=$ const, and the dashed line is a theoretical prediction for monopolarly charged granular gases. (b) Same as (a) but for the early stage of evolution of the viscoelastic $(\epsilon \neq$ const.) granular gas with charge exchange. The dashed line represents the theoretical prediction of Haff's law for a neutral viscoelastic granular gas. Here the notations $T$ and $T_{\mathrm{g}}$ are used interchangeably, and $t_{0}$ is some time scale of relaxation of the initial transience in the gas. The time scale $t_{c}$ marks a transition from Haff's law to inverse logarithmic decay of $T$. . . .

4.2 Snapshots of the granular gas showing the time evolution of the neutral system of viscoelastic particles (left column) and charged viscoelastic particles (center and right columns). Here time $t^{*}=t v_{\text {ref }} / d$, total number of monomers $N_{\text {mon }}=50016$ and the particle filling fraction in the system $\phi=0.076$. As the ratio of characteristic Coulomb to thermal energy $\mathcal{K}$ increases, the characteristic time for the emergence of clustering decreases, however, their growth rate is unchanged (see also Figs. 4.3 and 4.5). The clusters exhibit a relatively compact morphology in the charged system. .

4.3 Sticking of particles during clustering in the charged system. We observe a mechanism similar to collide-and-capture events observed in experiments on a falling granular stream by Lee et al. (2015). Particles stick together in clusters and exhibit pronounced persistence in each cluster over a considerable duration of time. This mechanism is not observed in the neutral system, where instead particles collide and separate. (a) Specific particles and their first neighbors are shown at different times. (b)-(d) The evolution of the number of contacts, $N_{\text {stick}}$, with time for the same particles shown in panel (a). The occasional fragmentation results in the fluctuation of $N_{\text {stick }}$ with time. . . . . . . . . . . . . . 
4.4 (a) The evolution of mean absolute charge, $\bar{q}=\frac{1}{N} \sum_{i=1}^{N}\left|q_{i}\right|$, for the viscoelastic model. The mean charge saturates as a consequence of continuous reduction in the granular temperature. The solid line is the theoretical prediction in Eq. (4.6). (b-d) Comparison of the cluster size distribution $N_{s}(s)$ for charged $(\mathcal{K}=5.0)$ and neutral granular gases at different times: (b) $t v_{\text {ref }} / d_{0}=50$; (c) $t v_{\text {ref }} / d_{0}=100$; (d) $t v_{\text {ref }} / d_{0}=150$, from an average over twenty independently initialized simulation runs. The dashed lines are the corresponding best fits. During the evolution of the granular gases, the slope of the distributions, or equivalently, the Fisher exponent decreases. However the charged gas exhibits a more rapid decrease of the slope indicating an enhanced cluster growth (see also Fig. 4.5). . . . . . .

4.5 During early stages of the growth, the temporal dependence of the average cluster size $S(t)$, from an average over twenty independently initialized simulation runs and corresponding best fit. Granular gas with collisional charging exhibit faster growth of clusters than for neutral system. (Inset) saturation of the growth exponent $z$ as $1 / t \rightarrow 0$ using the method of local slope [Eq. (4.11)]. A change in the ratio of characteristic Coulomb to thermal energy $\mathcal{K}$ do not alter the growth exponent $z$ and only influences the crossover time of initiation of clustering. . . . . . . . . . .

4.6 Comparison of the average cluster size $S(t)$ predicted by the Smoluchowski equation with the MD results. At $\beta=2$ (dashed line) the meanfield solution agrees reasonably well with the neutral gas, while at $\beta=3$ (solid line) the growth rate for the charged granular gas is recovered. (Inset) Comparison of the MSD of particles between the charged and the neutral gas. The sub-diffusion due to dissipation is further suppressed by the electrostatics, which in the mean-field approximation is modeled by increasing $\beta$. Here $S_{c}$ and $\tau_{c}$ are factors used to rescale the curves and plot them close to each other for the sake of comparison of the slopes. Here the non-dimensional time $t^{*}=t v_{\text {ref }} / d \ldots \ldots \ldots \ldots \ldots$

4.7 (a) The relaxation of the scaled velocity $\left(\tilde{v}=v /\left\langle v^{2}\right\rangle^{1 / 2}\right)$ distribution function towards the Maxwellian for neutral viscoelastic particles. This result from our simulations for neutral viscoelastic particles is consistent with the Sonine expansion for the time dependent distribution function (Brilliantov \& Pöschel, 2010) which depicts that the distribution relaxes back towards the Maxwellian for long time. (b) The time evolution of the distribution $f(\tilde{v})$ for a charged system, however, shows a behavior opposite to the neutral case: the distribution does not relax back to the Maxwellian. (Insets) Same results as in (a) and (b) but on a linear scale to highlight the shift of the most probable velocity for the charged granular gas. Here the non-dimensional time $t^{*}=t v_{\text {ref }} / d \ldots \ldots \ldots \ldots \ldots$

4.8 Scatter plot of the particles' charges and velocities at different times. As time progresses, a subpopulation of high velocity, nearly neutral particles develops (highlighted in the dashed ellipse). This subpopulation is expected to cause the occasional fragmentation of the local aggregates (see Fig. 4.3). Here $\tilde{q}=q /\left\langle q^{2}\right\rangle^{1 / 2}$ and $\tilde{v}=v /\left\langle v^{2}\right\rangle^{1 / 2}$. Here the nondimensional time $t^{*}=t v_{\text {ref }} / d \ldots \ldots \ldots \ldots \ldots$ 
5.1 Snapshots of the aggregating charged granular gas at different nondimensional times: (a) $t^{*}=19$, (b) $t^{*}=59$, and (c) $t^{*}=99$. Here clusters containing 10 particles or more $\left(N_{c l} \geq 10\right)$ are shown, and each color represents a different cluster. Clusters are identified on the basis of the monomer distances: if the centers of two particles are separated by a particle diameter, or less, they belong to the same cluster. See Methods for non-dimensional time $t^{*}=t v_{\text {ref }} / d_{0}$, Coulomb force strength $\mathcal{K}$, and other reference scales. . . . . . . . . . . . . . . . .

5.2 (a) When charged particles collide (particle group I), two possible pathways are considered in the kinetic theory depending on their velocities and charges: $(i)$ a typical inelastic collision (dissipation with velocity dependent coefficient of restitution) with charge exchange (particle group II) if the relative collision speed is above a threshold speed $\sqrt{b}$, and (ii) a collision that leads to the aggregation (with coefficient of restitution equal to zero) and merging of charges (particle group III), if the relative collision speed is below $\sqrt{b}$ and the particles are oppositely charged. The latter event introduces a size distribution of aggregates, which we further simplify in our kinetic theory by adjusting the size of all the particles (particle group IV). Thus, the particle size is assumed to remain monodispersed during the course of aggregation. Primed variables represent postcollision or post-aggregation values. (b) Coefficient of restitution $\epsilon\left(v_{i j}\right)$ in the present kinetic theory. Notice that the threshold $\sqrt{b}=\sqrt{\frac{2 k_{e}\left|q_{i} q_{j}\right|}{m d}}$ also evolves with time due to charge-exchange and aggregation events, and the coefficient of restitution is zero only if $\Theta\left(-q_{i} q_{j}\right) \Theta\left(b^{1 / 2}-v_{i j}\right)=1$. The above kinetic approach is compared with the MD simulations where the size distribution of aggregates is polydisperse. . . . . . . . . .

5.3 Evolution of temperature (a) $T$ of cluster population, (b) charge variance $\left\langle\delta q^{2}\right\rangle$ of cluster population, and (c) number density $n$ of cluster population, for different monomer filling fractions $\phi$ and charge strength $\mathcal{K}$. (d) The granular temperature, charge variance and average size of the cluster population during aggregation evolve in such a manner that their non-dimensional combination $\mathcal{B}(t)=k_{e}\left\langle\delta q^{2}\right\rangle /(T d) \leq 1$ (see also Fig. 5.4). Both temperature and charge variance of cluster population decay as power laws. The number density evolution, however, is highly dynamic and exhibits a non-monotonic behavior due to emergence of mesoscopic flow (see Methods for $\phi, \mathcal{K}, v_{\text {ref }}, d_{0}$ and mesoscopic flow). . . . . .

5.4 The granular temperature, charge variance and average size of the cluster population during aggregation evolve in such a manner that their nondimensional combination $\mathcal{B}(t)=k_{e}\left\langle\delta q^{2}\right\rangle /(T d) \leq 1$. This is not captured in the kinetic theory if only restitutive (no aggregation) collisions are considered. The granular MD simulations confirm the analytical results. (inset for different monomer filling fraction $\phi) . \ldots \ldots . . . . .$. 
5.5 (a-c) The scaling between cluster mass $m$ and their radius of gyration $R_{g}$, $m \sim R_{g}^{D_{f}}$ at different times, and (d) the time evolution of $\left\langle D_{f}\right\rangle$, thus obtained, for different filling fractions. The average fractal dimension in the aggregating charged gas varies across reported average values for ballistic cluster-cluster aggregation (BCCA, $\left\langle D_{f}\right\rangle \sim 1.94$ ) and diffusionlimited particle-cluster aggregation (DLPCA, $\left\langle D_{f}\right\rangle \sim 2.46$ ) (Blum, 2006; Smirnov, 1990). $t^{*} \equiv t v_{\text {ref }} / d_{0}$. Here the initial transience of the fractal dimension during the charge buildup phase is omitted. . . . . . . . . . .

5.6 The scaled charge distribution $f(\tilde{q})$ of individual particles obtained from typical MD simulation runs (dots) in the aggregated granular gas. The solid line is a Gaussian fit. Here $\tilde{q}=q /\left\langle\delta q^{2}\right\rangle^{1 / 2} \ldots \ldots$. . . . . . . . . . . 10

5.7 Individual comparisons of (a) temperature of cluster population $T$, (b) charge variance of cluster population $\left\langle\delta q^{2}\right\rangle$, and (c) number density of cluster population $n$, with the theoretical predictions. The granular temperature, charge variance and average size of the cluster population during aggregation evolve in such a manner that their non-dimensional combination $\mathcal{B}(t)=k_{e}\left\langle\delta q^{2}\right\rangle /(T d) \leq 1$ (main text). The number density evolution, however, is highly dynamic and exhibits a non-monotonic behavior due to emergence of mesoscopic flow, marked by a transition in the rate of increase of Mach number $\mathcal{M}$, shown in (c) inset. The numbers in (c) inset are the power law exponents of the time dependence of $\mathcal{M}$. The short-hands res and agg denote restitution and aggregation respectively. .

5.8 Growth of the average cluster size $S$, and the size of the largest cluster with time. Here $S$ is computed from the mass-size relationship $S \sim\langle m\rangle^{1 /\left\langle D_{f}\right\rangle}$. The average mass is defined as $\sum_{k} m_{k}^{2} N_{m}(m) / \sum_{k} m_{k} N_{m}(m)$. Here $\left\langle D_{f}\right\rangle$ is the average fractal dimension, $N_{m}(m)$ is the mass distribution of the aggregates, and the index $k$ runs over all the aggregates. See also Figs. 4.5 and $4.6 \ldots \ldots$. . . . .

6.1 The change in the eigenvalues $\lambda_{i}(k)$ as the value of the non-dimensional parameter $\mathcal{R}$ is increased from 0 (a) to 0.9 (f). The case $\mathcal{R}=0$ corresponds to a neutral granular gas (solid lines), where one eigenvalue is positive and signifies heat mode of clustering instability. As $\mathcal{R}$ is increased, the eigenvalue corresponding to the heat mode is suppressed, and the other two eigenvalues may become equal and positive (dashed lines). At certain $\mathcal{R}$, these two eigenvalues take over [e.g. in subplot (e) and (f)].

6.2 The change in the eigenvalues $\lambda_{i}(k)$ as the value of the coefficient of restitution $\epsilon$ is decreased from 0.8 (a) to 0.3 (f) at a fixed $\mathcal{R}=0.9$. With increasing dissipation, the critical value of $k$ for a charged granular gas (dashed lines) is decreased from its neutral counterpart (solid lines). . . .

6.3 The value of the quantity $\frac{\mathcal{R}}{\left|\epsilon^{2}-1\right|}$ as a function of the critical value of $k$. The line with symbols corresponds to an unstable mode which will be excited first in the charged gas. Up to some value of $\frac{\mathcal{R}}{\left|\epsilon^{2}-1\right|}$, the heat mode will initiate the instability, while after that the sound mode will be the dominant mechanism. As $\frac{\mathcal{R}}{\left|\epsilon^{2}-1\right|}$ increases, the change from the heat mode to the sound mode is abrupt. 
6.4 If charge is separated over some length scale, it is expected that the electrostatic origin of instability in the regions close to $\rho_{\mathrm{q}}=0$ will be nonlinear in principle. In the regions which can be described with some charge density bias $\rho_{\mathrm{q} 0}$, the electrostatic origin of instability can be linear. . . . . . . . . . . . . . . . . . .

7.1 The masses of aggregates, $m$, versus the magnitude of net dipole moment of aggregates, $\mu \equiv\left|\sum_{i} \boldsymbol{\mu}_{i}\right|$ [defined in Eq. (7.1)]. Here the index $i$ runs over all the monomers in a given aggregate. Three panels (a), (b) and (c) are for different non-dimensional times $t^{*}=140.0, t^{*}=380.0$ and $t^{*}=600.0$ respectively. Two colors are for different non-dimensional polarizability $\mathcal{A}$. The results indicate that larger size aggregates have relatively small magnitudes of net dipole moment. From this, it is expected that the induced polarization should have relatively more influence on the morphology of the smaller aggregates compared to the larger ones. . . . .

7.2 The average fractal dimension $\left\langle D_{f}\right\rangle$ with time, for different nondimensional polarizability $\mathcal{A}$. The average fractal dimension decreases as the polarizability increases, and this effect is more pronounced at early times during the aggregation process. The initial transients during the charge-buildup $\left(t^{*} \lesssim 200\right)$ are excluded and the dynamics after charge saturation on the monomers is compared in the discussion [subsection 7.1.2].134

7.3 The average cluster size $S \sim\langle m\rangle^{1 /\left\langle D_{f}\right\rangle}$, with time, for different nondimensional polarizability $\mathcal{A}$. Here $\langle m\rangle=\sum_{k} m_{k}^{2} N(m) / \sum_{k} m_{k} N(m)$,

$7.4 \quad$ The average kinetic energy and the granular temperature of a granular gas (a) without, and (b) with the effect of viscous friction due to the random interstitial fluid velocity (see Eq. (3.53) in chapter 3 ). Here polarizability $\mathcal{A}=0.075$ in both (a) and (b), and the Stokes number $\mathcal{S}=0.0$ in (a) while $\mathcal{S}=0.05$ in (b). In the second case, a steady state granular temperature is attained after the granular temperature reaches the order of energy input due to random interstitial fluid velocity. This case is closer to practical scenarios of dust aggregation where the influence of interstitial medium is important. In the steady state time window, the mass versus dipole moment of the fractal aggregates is studied and presented in Fig. 7.5. Here $v_{i}$ is the grain speed and $V$ is the mean velocity of grains in the vicinity

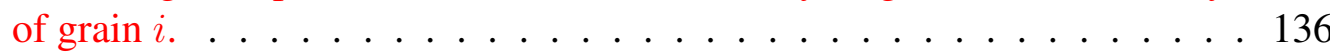

7.5 Same as in Fig. 7.1 but for fixed $\mathcal{A}=0.075$ and for different Stokes number $\mathcal{S}$. Three panels are for three different times. In case of $\mathcal{S}=0.05$, the aggregates with only $N_{\text {mon }} \leq 5$ are able to survive because of the fragmentation caused by random interstitial velocities. [see subsection 7.2] 
7.6 The surviving aggregates in a polarized granular gas, when the grains experience drag due to random interstitial velocities [section 7.2]. The polarization forces result in the formation of small chains, however, the sizes of these chains are limited by the random drag to only few monomers. Each aggregate is represented by different color, and aggregates having three or more monomers are shown $\left(N_{c l} \geq 3\right)$. The Stokes number $\mathcal{S}=0.05$ and the normalized polarizability $\mathcal{A}=0.075$. See also Fig. 7.5 . . . . . . . 141

8.1 Different grain size regimes and corresponding mechanisms of growth. The results from this thesis support the following school of thought (Spahn \& Seiß, 2015): in the grain size regime $d_{0} \sim \mathrm{mm}-\mathrm{cm}$, electrification and polarization of grains might be a dominant mechanism, which is capable of producing aggregate sizes sufficient for subsequent gravitational collapse $\left(d_{0} \gtrsim \mathrm{m}\right)$. For grain sizes $d_{0} \lesssim \mu \mathrm{m}$, cohesive forces such as Van der Waals are important in the growth process. . . . . . . . . 147

A2.2 Overall granular molecular dynamics algorithm, and incorporation of charge-exchange and induced polarization . . . . . . . . . . . 151

A5.3 Typical time evolution of the hydrodynamic equations (6.26) introduced in chapter 6 . The left and right-hand side panels, respectively, show the macroscopic charge and mass density fields, normalized by some appropriate reference values. (a) The initial charge density (top left) is set to emphasize a possible scenario of charge separation, as schematically shown in Fig. 6.4. (b) It is shown that the regions (marked by XX) for which the terms linear in $\delta \tilde{\rho}_{\mathrm{q}}$ or $\delta \tilde{\varphi}$ in chapter 6 are expected to vanish, indeed develop high mass densities (bottom right, marked by XX). The results from the linear stability analysis presented in chapter 6 are thus valid away from these regions where a finite $\rho_{\mathrm{q} 0}$ approximation is reasonable. Credits: In this particular figure, the hydrodynamic code was developed by Dr. Mathias Hummel (Hummel et al. , 2016), while the electrostatic part was added by Chamkor Singh. . . . . . . . . . . . . . . . . . . . . . 


\section{List of Tables}

3.1 Different models considered for the charge-exchange currents $I_{j i}$. Here $d_{0}$ is the grain diameter, $q_{i}$ is the charge on a grain $i$, and $r_{i j}$ is the distance between grains $i$ and $j \ldots \ldots \ldots \ldots$. . . . . . . . . . 51

5.1 Expressions of the coefficients in Eq. (5)-(7) in the main text. Here $m$ and $d$ are the mass and size of the aggregates. The material constant $C_{\epsilon}$ is from Eq. (5.31) and influence the viscoelastic properties of the particles, while $C_{\Delta q}$ and $\eta$ are from Eq. (5.28) and influence the charge buildup. Other notations are as described in the main text. . . . . . . . . . . . 112

5.2 Simulation parameters used in chapter $5 \ldots$. . . . . . . . . . . 113

5.3 Reference values in SI units for conversion of non-dimensional results to laboratory relevant values. . . . . . . . . . . . . . . . . . . . 114 

"Most of the world is of great roughness and infinite complexity. However, the infinite sea of complexity includes two islands of simplicity: one of Euclidean simplicity and a second of relative simplicity in which roughness is present but is the same at all scales."

Benoît Mandelbrot 



\section{Chapter 1}

\section{Introduction}

\subsection{Motivation}

Granular media are collections of athermal macroscopic grains. Flows of granular materials are ubiquitous in nature, industry and in our everyday life. There is a wide range of phenomena which involves granular matter in one way or the other. From the sand on a beach to the rings of Saturn, from the extraction of minerals to the salt and pepper in our kitchen, and from pharmaceutical to the medicine industry, the appearance of granular matter is unavoidable. As an example on astronomical scales, Fig. 1.1 shows the Pillars of Creation of the Eagle's Nebula, captured by the Hubble space telescope. These clouds of cosmic dust in the pillars, although surrounded and influenced by gas and radiation, are one example of granular structures having the size of the order of light years. On the other hand, Fig. 1.2 shows typical examples from medicine, mining, agriculture, and mineral industries. Given that granular matter is omnipresent, the rigorous understanding of their dynamics is vital, both for the industry as well as for natural curiosity.

The granular flows are usually characterized by inelasticity and friction during collisions of constituent grains. Thus they dissipate energy and require external or interstitial driving for sustained motion. Densely packed granular materials may behave like solids (de Gennes, 1999). Under large enough forcing, they may flow and show a transition to a liquid-like behavior (Campbell, 1990). Even larger driving, such as vibratory motion, may fluidize the grains resembling a gaseous phase. Exposed to different conditions, granular materials display various patterns, for example, dunes and ripples 

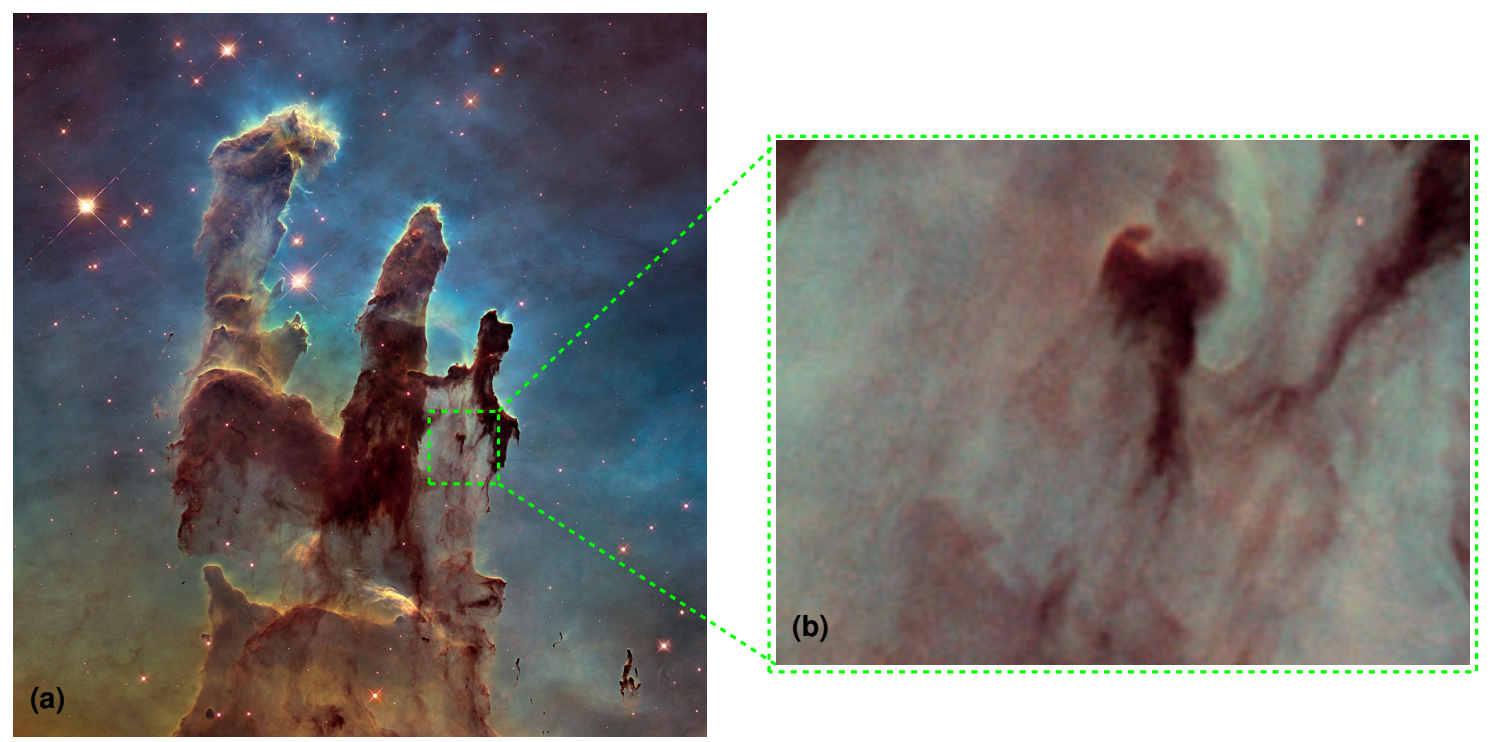

Figure 1.1: (a) The Pillars of Creation of Eagle's nebula observed by the Hubble space telescope, and (b) exaggerated view of a cosmic dust cluster in the nebula. Original image source: ESA/Hubble. weblink: https://www.spacetelescope.org/images/heic1501a.

in deserts, Chladni figures in vibrated granular layers, fingering in planar avalanches, etc (Aranson \& Tsimring, 2006). The flows of granular matter are also a great example of fluid-mechanical phenomena which is distinctive, but in some sense still analogous, to the flows of ordinary liquids and gases (Haff, 1983; Jaeger et al. , 1996).

A particularly interesting class of granular matter is its dilute phase which is initially driven but evolves with no external forces, known as a granular gas. Although mass and momentum are conserved during collisions, energy dissipation ${ }^{1}$ in granular gases leads to complex dynamic facets. These include appearance of clusters, vortex formation, and power-law decay of particle velocity fluctuations (Goldhirsch \& Zanetti, 1993; McNamara, 1993; Brito \& Ernst, 1998; Brey et al. , 1999; Brilliantov et al. , 2004; Nie et al. , 2002). Furthermore, a non-zero dissipation produces other intriguing features such as anomalous diffusion (Bodrova et al. , 2016; Brilliantov \& Pöschel, 2000a), and dissimilarity between ensemble-averages and long-time averages of observables (non-ergodicity) (Bodrova et al. , 2015).

The dynamics of neutral granular gases have been extensively studied in the last few decades. For granular gases of dielectric grains, it is highly expected that the grains attain electric charges due to contact or triboelectrification during mutual collisions. This aspect

\footnotetext{
${ }^{1}$ The kinetic energy dissipated during a collision can be specified in terms of the coefficient of restitution, which is elaborated in Fig. 3.1 in chapter 3.
} 


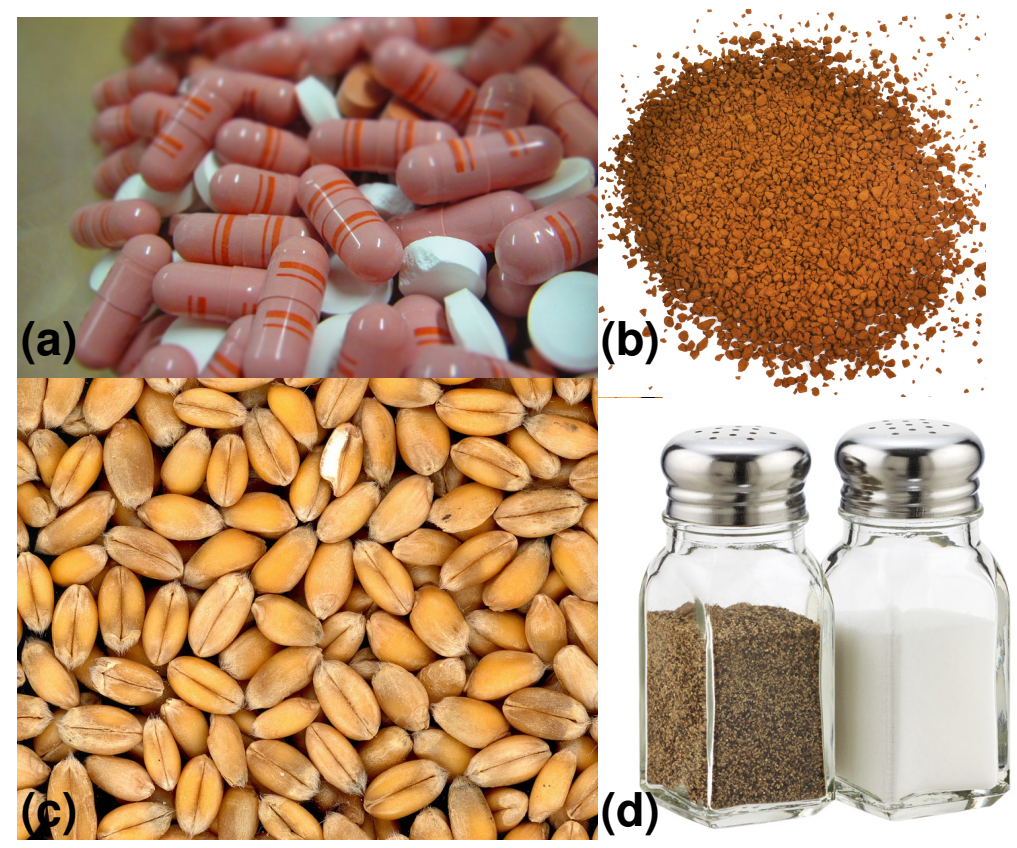

Figure 1.2: Typical examples from medicines, mining, agriculture and mineral industries. (a) medicinal tablets, ${ }^{1}$ (b) ferric-oxide, ${ }^{2}$ (c) raw food grains, ${ }^{3}$ and (d) salt and pepper used in our everyday life. ${ }^{4}$

is rarely addressed in the context of granular gases. In addition to triboelectrification mechanisms, the additional degrees of freedom originating due to grain polarizability further complicate the problem. Furthermore, studies of collisionally or triboelectrically charged granular gases may help to reveal some dynamic aspects of the natural systems, such as electrified volcanoes and sand storms [Fig. 1.3].

The dynamics and pattern formation in granular gases under such scenarios, or at least close to such scenarios, is the topic of this thesis. In the next section and subsections therein, literature relevant to the scope of the thesis is first reviewed. A summary of the literature is provided thereafter. Finally, the extent and scope of the present thesis are discussed.

\footnotetext{
${ }^{1}$ Image source: www.pixabay.com/photos/tablets-pills-medicine-disease-700670

${ }^{2}$ Image source: www.modernaquatix.com/bulk-gfo-granular-ferric-oxide

${ }^{3}$ Image source: www.pixabay.com/photos/food-cereals-wheat-grains-plant-4509

${ }^{4}$ Image source: www.amazon.com/Salt-Pepper-Shakers-Glass-Clear/dp/B06XH11C7K.
} 


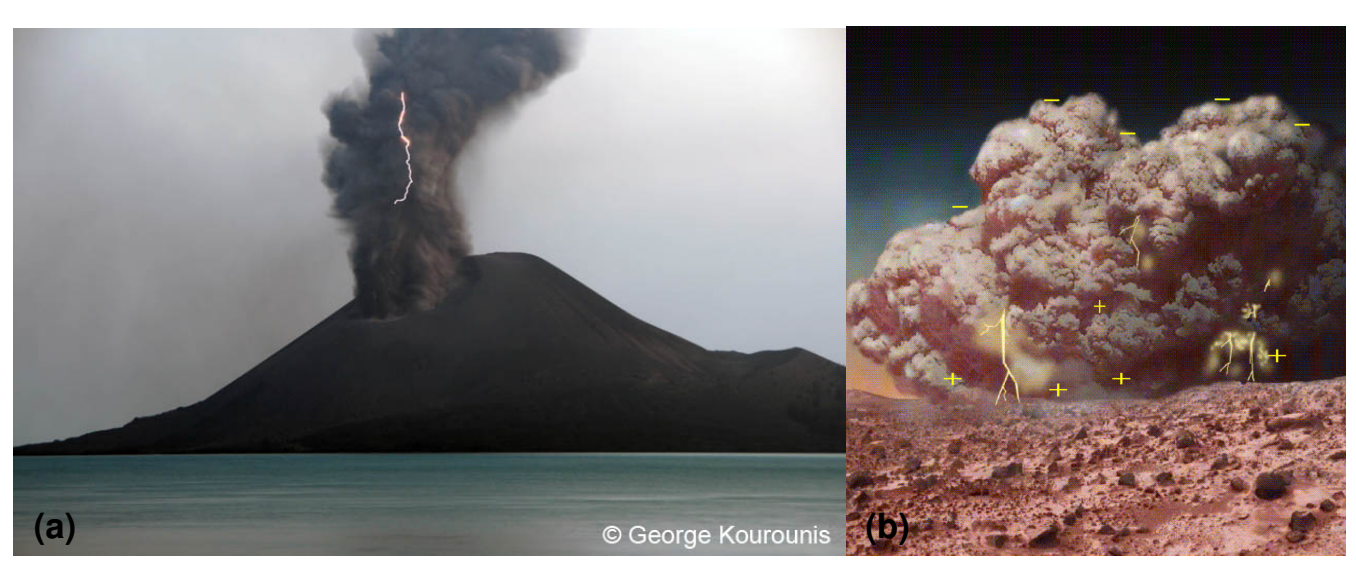

Figure 1.3: (a) Lightening due to static electrification of volcanic ash particles, ${ }^{1}$ and (b) an artist's impression of electrically charged dust strom on Mars. ${ }^{2}$

\subsection{Literature review}

Electrostatics plays a vital role in a wide range of natural as well industrial processes. Few most common examples involving electrification are:

(i) Charge separation in clouds (Saunders, 2008); charging of ocean droplets, wind blown dust, earthquake lightening, volcanic plumes and even sand storms.

(ii) Spray paints, precipitation of fly-ash using corona discharge, xerography, dielectrophoresis, electrostatic separation of minerals (Moore, 1972).

(iii) The transportation of powders, sugar, flour or pharmaceuticals through ducts.

(iv) Use of electrostatic probes to measure particulate mass flow rates in conduits (Cheng \& Soo, 1970).

In the following subsections, the present understanding and relevant literature of electrification in particle systems are classified into five categories: (i) granular frictional or tribo and contact electrification, (ii) charged suspensions, powders, colloids and dusty plasma, (iii) aggregation of charged particles and astrophysical applications, (iv) collective effects in flows of charged particles, and finally $(v)$ charged granular gases and their scope.

\footnotetext{
${ }^{1}(\mathrm{c})$ George Kourounis.

${ }^{2}$ Image source: www.nasa.gov/images/content/151990main_elec_dust_storm_lgweb.jpg
} 


\subsubsection{Granular frictional or tribo, and contact electrification}

The existence of electric charges is known from as early as $600 \mathrm{BC}$. It was observed that a mineral called amber was able to attract light pieces of material, such as feather, when rubbed with fur. The common term to describe this frictional electricity is triboelectrification. However, later it was understood that charge transfer can be achieved by mere contact between two surfaces without relative motion. The extent of charge transfer, however, might depend on the motion. To understand the contact or triboelectrification and its effects on flows of insulator particles, extensive research has been performed in the past. Below some recent studies are listed.

For contact between metal and glass surfaces, Lowell (1991) found that the interfacial electric field is modified by conduction of charges, which in turn has an effect on the charge transfer. Zhang et al. (2015) investigated the effect of external electric field and humidity on contact electrification of insulators. The researchers suggested that the surface can adsorb small amounts of water from the surrounding air, and the electric field subsequently separates positive ions from negative ions. During the contact of two insulators, they claim ions of one sign on one surface neutralizes the ions of opposite sign on the second surface, leaving both the insulator surfaces with net charges after the contact. Before this, theoretical efforts have been made to describe the effects of humidity/water content on contact triboelectrification of sand particles (Zheng et al. , 2014; Gu et al. , 2013). Yoshimatsu et al. (2016) studied the dynamics in fluidized granular bed under an external electric field applied parallel to gravity. The grains in this study were charged upon collisions using a mechanism proposed by Pähtz et al. (2010). According to this mechanism, the grains become polarized in the external field and the charge transfer takes place due to a neutralization event during the contact. The mechanism requires the presence of an external electric field. In a later study, Yoshimatsu et al. (2017) proposed a multipole grain model with self-charging of grains to study granular beds. The mechanism of grain charging in this study depended on polarizability and a neutralization efficiency. The researchers found that initially, a perturbative charge could grow exponentially in time. Among theoretical studies, $\mathrm{Hu} \&$ Xie (2016) proposed a model for the probability distribution function (PDF) of net charge transfer specific to a sand-bed collision process. Matias et al. (2018) theoretically studied mechanical equilibrium of a number of charged 
grains inside individual aggregates and found that grains charged with the same sign may also stay together due to induced polarization. In recent experiments, Schella et al. (2017) showed that tribocharging changes the particle coordination number in a shaken dense binary sphere packing. Haeberle et al. (2018) found charge distributions with exponential tails of particles undergone a single or multiple collisions. Shinbrot et al. (2017) proposed that the charging of grains undergoing repeated contacts occur via the development of local charge domains on the surface of grains, and the growth of these charge domains is exponential, although a slower transient growth may also be a possibility. Shinbrot et al. (2018) also found that the grain charging decreases as contact frequency increases if the discharging kinetics were rapid than the charging kinetics. The proposition was backed by granular bed experiments. The study visualized the charge patterns on the surface of a celluloid ball and found that the point of contact retains a minimum amount of charge. The authors suggested that the understanding of the kinetics of charge transport on the surface itself needs further investigations. Lee et al. (2018) developed a novel experimental technique to track the charge transfer between a single sub-millimeter sized particle with a flat surface based on acoustic-levitation. The charge transfer between surfaces found to be dependent on the hydrophobicity of the contacting surfaces.

\subsubsection{Charged suspensions, powders, colloids, and dusty plasma}

There are other charged particulate systems from which some analogies can be drawn, e.g., charged powders, colloids, and dusty plasma. Charge separation and particle size segregation were studied by Konopka \& Kosek (2017) for elastic particles with properties of polyethylene powder. Dammer \& Wolf (2004) found universal scaling for the size distribution for an equally charged particle suspension in a thermal bath, using the Smoluchowski aggregation equation. Another physical system close to granular gases is the dusty plasma, where the particles attain charge from the surrounding ionized environment (Matthews \& Hyde, 2004; Ma et al. , 2013; Horanyi \& Goertz, 1990). In such a setup, the dust grains may charge up due to primary plasma currents, secondary electron emission and/or photoemission (Ma et al. , 2013), and collisional or triboelectric charging is typically not considered. The charges attained by aggregates in plasma may correlate to the structural properties of the aggregates (Ma et al. , 2013). There is even the possibility 
of gelation or runaway-growth of aggregates (Matthews \& Hyde, 2004). Application of Smoluchowski's mean field approach has shown enhanced coagulation if the small grains in plasma are charged oppositely to the big ones (Horanyi \& Goertz, 1990). Matthews et al. (2018) theoretically studied dust aggregates in a plasma environment. The aggregates were prone to charge fluctuations due to the random attachment of small amounts of elementary charges from the plasma. Authors used a master equation to describe the time evolution of the PDF of the set of elementary charges attached to patches on the surface of the aggregate grains. In colloids, the fractal dimension of colloidal aggregates is known to be a decreasing function of dipolar forces (Lebovka, 2012).

\subsubsection{Aggregation of charged particles and astrophysical applica- tions}

Particle aggregation processes are a wide branch of study, ranging from nano-sized colloidal coagulation to formation of protoplanetary bodies. Mechanistically, the aggregation processes are classified based on the type of motion or trajectories of particles/aggregates and the probabilities that a given collision between two particles/aggregates will lead to sticking. Relevant to these processes, the following theoretical models need to be mentioned:

(i) Diffusion limited aggregation (DLA), also known as rapid aggregation, where the trajectories of particles/aggregates are typically Brownian or random, and the sticking/attachment probabilities are high.

(ii) Ballistic aggregation (BA), where the trajectories are straight.

(iii) Reaction limited aggregation (RLA), also know as slow aggregation, where the sticking/attachment probabilities are low.

(iv) Cluster-cluster aggregation (CCA), where two aggregates/clusters join to make a new aggregate.

(v) Particle-cluster aggregation (PCA), where a single particle/monomer joins with an aggregate/cluster to make a new aggregate. 
(vi) Eden like aggregation (ELA), where single particles attach on the periphery of the growing aggregate.

In addition to the above mechanisms, restructuring of clusters might also happen during the growth process. This restructuring kinetics might lead to a reversible (where fragmentation is permissible) or irreversible growth (where eventually one single large cluster appears).

In the context of aggregation of electrified particles, some recent studies have stimulated significant interest in the problem. The most important in our opinion are listed below. Ivlev et al. (2002) studied the aggregation of charged micro-particles in an ambient gas using the Smoluchowski aggregation equation for a mass-charge joint probability distribution function. The analytical results indicated that a gelation or run-away growth may occur if charge-dipole interaction is also taken into account. These gelation events were also observed in a later experimental study, where charged micro-particles were injected in a low-pressure neutral gas environment (Konopka et al. , 2005) with sufficient initial number densities. Space shuttle microgravity experiments with sub-millimeter particles conducted by Marshall et al. (2005) revealed that electrostatic forces lead to the formation of filamentary, and under certain conditions, larger size loosely packed aggregates. The experiments indicate that dipolar interactions play a significant role. Lee et al. (2015) experimentally showed charging and aggregation in freely falling granular streams. The experiments showed millimeter-sized charged particles to follow Kepler-like orbits in their mutual electrostatic field, bouncing on each other before forming aggregates. The aggregation due to electrostatics is also found to be prevalent for mineral grains with a chemical composition similar to that which are found in the sand of Titan (Harper et al. , 2017). The resulting effect would be that the threshold velocity of the wind flowing over the surface of the Titan's sand has to be larger to cause any significant transport.

\subsubsection{Collective effects in flows of charged particles}

The collective effects, and pattern formation, caused by grain contact or triboelectrification in a many-body granular system (dense or dilute) remains largely unexplored. Below are some of the recent studies which have touched this aspect. The effect of particle charging and polarization on particle-laden flow in a vertical pipe under gravity, in the 
absence of dissipation during particle-particle collisions, is studied by Kolehmainen et al. (2018a). In this study, which is specific to wall-bounded flow, researchers found qualitative changes in the particle flow characteristics, such as meandering or sheathing flow regimes. Di Renzo \& Urzay (2018) studied inertial charged particles in turbulence. The particles experienced drag and electrostatic forces, and the particle-particle collisions were neglected. Half of the particle population was considered to carry constant value positive charge, and the rest was carrying constant value negative charge. The study revealed the generation of mesoscopic electric fields. Nordsiek \& Lathrop (2015) studied the collective behavior of grains of different materials shaken inside a cell between two conductive plates. The electrical potential across the two plates was measured. Partial segregation events were observed in the presence of electrification of grains. The observations were found to be unaltered by the particle size, shape or material. The study suggested that structures forming at large scale and long-range interactions are important to understand the electrification phenomena in general.

\subsubsection{Charged granular gases and their scope}

Very few studies exist where the granular gases have been augmented with long-range potentials. Some of the known outcomes are listed as follows. Müller \& Luding (2011) studied the granular temperature using pseudo-Liouville operator formalism and MD simulations for attractive (self-gravity) and repulsive $r^{-1}$ potentials. For short-ranged attractive potentials, Murphy \& Subramaniam (2015) studied the deviation of granular temperature from the Haff's law up to short times and noticed velocity correlations arising due to monomer sticking events. Other than self-gravity, attractive non-contact potentials between grains are expected to enhance clustering, while the opposite is true for repulsive potentials (Gonzalez et al. , 2014). Granular temperature decays inverse logarithmically in a monopolar and equally charged granular gas (Scheffler \& Wolf, 2002; Takada et al. , 2017).

It is important to note that in most of the above studies in this subsection, the homogeneous cooling state (HCS) of the granular gas was the primary focus. The structure formation, the morphology of the clusters, and the collective effects emerging out of the aggregation processes in a charged (and polarized) granular gas are still largely unexplored. 
The collective behavior and pattern formation in heterogeneously charged particulate systems is in general an inadequately understood phenomenon. Although analogies can be drawn from other charged systems (such as charged colloids, dusty plasma, and experimental works on protoplanetary dust aggregation, as discussed in previous subsections) there is ample scope to theoretically describe the pattern formation in these many-body systems, beginning from the microscopic collision level where both the dissipation and tribo/contact electrification play key roles.

\subsection{Extent, scope and organization of the thesis}

This thesis is focused on understanding the aggregation and pattern formation in heterogeneously charged, globally charge conserving, and initially dilute granular gases; the gas is charged with simplified but physically valid charge-exchange recipes and the subsequent effects of the long-range forces on the dynamics and morphology are studied. This theoretical setup closely replicates, or at least targets, the charged dust agglomeration scenarios. Canonical observables, such as growth rates of the average cluster size, average fractal dimension, granular temperature, and charge variance are computed using granular molecular dynamics simulations. In addition, the size, velocity and charge distributions are also studied. To systematically understand the aggregation and patterns, different approaches are used in the thesis, namely

(i) Smoluchowski's mean field equation with modified aggregation kernel,

(ii) kinetic theory with modiefied collision integral,

(iii) molecular dynamics simulations, and

(iv) linear stability analysis of the hydrodynamic equations for the onset of pattern forming instability.

The forces acting on macroscopic grains in granular media are due to mutual mechanical contacts or cohesion/surface forces, augmented by interactions with interstitial medium; additionally, there might be non-contact forces such as gravitational or electrostatic. Let us consider these forces one by one under a dilute limit, that is $\bar{r} \gg d_{0}$, where 
$d_{0}$ is typical grain size and $\bar{r}$ is the mean separation between grains. The mechanical contact forces in granular media are inevitable. The accelerations of grains due to interstitial fluid will typically scale as $\sim v / \tau_{\text {fric }}$, where $v$ is the velocity of grains relative to fluid and $\tau_{\text {fric }}$ is some friction time scale between grains and the fluid. As long as this time scale is large enough, the interstitial medium can be neglected. The gravitational forces will typically scale as $G m^{2} / \bar{r}^{2}$, where $m$ and $G$ are the grain mass and the gravitation constant respectively. No matter how small relative to other forces, gravitational forces are never zero. The electrostatic forces are active if the grains are charged and will scale as $k_{e} q^{2} / \bar{r}^{2}$, where $q$ and $k_{e}$ are the typical charge on grains and the Coulomb's constant respectively. The electrostatics is expected to dominate gravitation only if $k_{e} q^{2} \gg G m^{2}$, or $q / m \gg \sqrt{G / k_{e}}$. Thus in vacuum, the charge by mass ratio should be much greater than $\sqrt{G / k_{e}} \approx 8.6 \times 10^{-11} \mathrm{C} \mathrm{kg}^{-1}$ for electrostatics to be more significant than the gravitation. Under this, and using the definitions of grain material's mass density $\rho_{\mathrm{m}}$ and surface charge density $\rho_{\mathrm{q}}$, it can be estimated that for electrostatics to be dominant over the gravitation in vacuum

$$
d_{0} \ll \frac{\rho_{\mathrm{q}}}{\rho_{\mathrm{m}}} \times \sqrt{\frac{k_{e}}{G}}
$$

Putting typically measured charge densities $\left(\approx 10^{-6} \mathrm{C} \mathrm{m}^{-2}\right){ }^{1}$ mass density of silica $\left(\approx 3800 \mathrm{~kg} \mathrm{~m}^{-3}\right),{ }^{1}$ and values of the Gravitational and Coulomb's constant, it can be estimated that electrostatic will be dominant over gravity if

$$
d_{0} \ll 1 \mathrm{~m} \text {. }
$$

The results in this thesis should qualitatively apply to charged granular gases with the above condition on the monomer sizes, together with the condition of initially dilute gas

$$
d_{0} \ll \bar{r}
$$

\footnotetext{
${ }^{1}$ Lee et al. $(2015)$ measured $\approx 10^{6}$ elementary charges on $\approx 300 \mu \mathrm{m}$ diameter particles, which indicates the surface charge density $\approx 10^{-6} \mathrm{C} \mathrm{m}^{-2}$.
} 
The grains are considered athermal, which under normal conditions roughly implies that

$$
d_{0} \gg 1 \mu \mathrm{m}
$$

To replicate contact electrification, the charge-buildup models used in this thesis primarily rely on experimental observations and do not target to resolve hard condensed matter details of the charge transfer processes.

The structure of the thesis is the following. The kinetic framework and the derivation of the mean field Smoluchowski equation are described in chapter 2. The granular molecular dynamics, treatment of long-range forces and polarization, treatment of chargeexchange currents is presented in chapter 3. Thereafter chapter 4 deals with the results for early-stages of aggregation, the mean field prediction of the aggregate growth rates, and the effects of electrostatics on velocity distribution. Chapter 5 contains the study of fractal structures and development of rate equation for macroscopic quantities during the aggregation. Chapter 6 deals with the linear stability analysis of the hydrodynamic description under certain limits and discusses the hydrodynamic modes involved at the onset of the inhomogeneous state. Thereafter preliminary results for induced polarization and viscous friction are presented in chapter 7 . Finally, the conclusions, outlook, and possible extensions are summarized in chapter 8 . 


\section{Chapter 2}

\section{Theoretical framework}

\subsection{Statistical distribution function}

Let us define a probability distribution function $f(\mathbf{r}, t ; \mathbf{v}, q)$, which describes the positions $\mathbf{r}$, velocities $\mathbf{v}$, and charges $q$ of equal sized spherical particles of the gas in their phase space, at a time $t$. Let us consider that the distribution function is normalized such that the product $f d \mathbf{r} d \mathbf{v} d q=d N$ is the mean number of particles in the phase space volume element $d \mathbf{r} d \mathbf{v} d q$. The particles in $d \mathbf{r} d \mathbf{v} d q$ have values of $\mathbf{r}, \mathbf{v}$ and $q$ in the ranges $d \mathbf{r}, d \mathbf{v}$ and $d q$ respectively. Integration over the entire phase space provides the normalization condition

$$
\int_{q} \int_{\mathbf{v}} \int_{\mathbf{r}} f(\mathbf{r}, t ; \mathbf{v}, q) d \mathbf{r} d \mathbf{v} d q=N
$$

where $N$ is the total number of particles in the gas. Instead of mean number of particles in the element $d \mathbf{r} d \mathbf{v} d q$, it is useful to consider the spatial number density $f d \mathbf{v} d q=\frac{d N}{d \mathbf{r}}$. In this way the spatial description is separated from the other phase variables, and the integration now provides

$$
\int_{q} \int_{\mathbf{v}} f(\mathbf{r}, t ; \mathbf{v}, q) d \mathbf{v} d q=n(\mathbf{r}, t) .
$$

Here $n$ is the mean spatial number density of the particles at $(\mathbf{r}, t)$.

In the above definition of the mean or macroscopic number density $n$, the linear size of the spatial volume element $d \mathbf{r}$ is considered much smaller than the characteristic linear 
size $L$ of the problem, while still large in comparison to the particle mean free path $\bar{r} \sim$ $\frac{L}{N^{1 / 3}}$. The volumes $d \mathbf{r}$ contain many particles and the particle number fluctuations in $d \mathbf{r}$ are negligible relative to the mean number of particles in $d \mathbf{r}$. In this study, the particle charge $q$ is explicitly considered as additional phase space variable. Explicit consideration of the charge dynamics will result in a modified Boltzmann equation, which is described in the next section.

\subsection{Boltzmann equation for a charged granular gas}

The time evolution of the distribution function $f(\mathbf{r}, t ; \mathbf{v}, q)$ is given by the Liouville's theorem, which states that

$$
\frac{d f}{d t}=\frac{\partial f}{\partial t}+\sum_{k} \dot{X}_{k} \frac{\partial f}{\partial X_{k}}=0
$$

Here $X$ are the phase space variables. Using the set $X=\{\mathbf{r}, \mathbf{v}, q\}$, the Liouville's theorem can be written as

$$
\frac{\partial f}{\partial t}+\mathbf{v} \cdot \frac{\partial f}{\partial \mathbf{r}}+\dot{\mathbf{v}} \cdot \frac{\partial f}{\partial \mathbf{v}}+\dot{q} \frac{\partial f}{\partial q}=0
$$

The accelerations $\dot{\mathbf{v}}$ of the particles might be due to internal (electrostatics in this case) and external body force fields, or due to the collisions. The last term on the left-hand side (LHS) signifies the changes in the distribution function produced by the charge exchange events during collisions.

The charged particles experience body forces due to the mutual electrostatic field. Let us consider that the electric field at the location of a particle is e. This microscopic electric field $\mathbf{e}$ at the location of a particle may be expressed as the sum of a mean electric field $\mathbf{E}$, and a fluctuating part $\mathbf{e}^{\prime}$ due to the nearby encounters with the other charged particles, i.e., $\mathbf{e}=\mathbf{E}+\mathbf{e}^{\prime}$. Thus the acceleration due to body force is $\dot{\mathbf{v}}^{\text {body }}=\frac{q}{m_{0}}\left(\mathbf{E}+\mathbf{e}^{\prime}\right) ; m_{0}$ in the monomer mass. The contribution to the acceleration caused by the collisions is denoted by $\dot{\mathbf{v}}^{\text {coll }}$. The acceleration due to external body force fields (for example gravity 
or external magnetic field) is considered zero. Using these, Eq. (2.4) is rewritten as

$$
\frac{\partial f}{\partial t}+\mathbf{v} \cdot \frac{\partial f}{\partial \mathbf{r}}+\frac{q \mathbf{E}}{m_{0}} \cdot \frac{\partial f}{\partial \mathbf{v}}=-\dot{\mathbf{v}}^{\text {coll }} \cdot \frac{\partial f}{\partial \mathbf{v}}-\frac{q \mathbf{e}^{\prime}}{m_{0}} \cdot \frac{\partial f}{\partial \mathbf{v}}-\dot{q} \frac{\partial f}{\partial q}
$$

As discussed, the right-hand side (RHS) in the above equation arises due to grain collisions, electric field fluctuations (or Coulomb collisions), and charge exchange events during collisions. Here the RHS is termed as the modified collision integral

$$
I^{\text {coll }}=-\dot{\mathbf{v}}^{\text {coll }} \cdot \frac{\partial f}{\partial \mathbf{v}}-\frac{q \mathbf{e}^{\prime}}{m_{0}} \cdot \frac{\partial f}{\partial \mathbf{v}}-\dot{q} \frac{\partial f}{\partial q}
$$

The mean electric field E must satisfy the averaged Maxwell's electrostatic equations

$$
\begin{aligned}
& \frac{\partial}{\partial \mathbf{r}} \times \mathbf{E}=0 \\
& \frac{\partial}{\partial \mathbf{r}} \cdot \mathbf{E}=4 \pi \rho_{\mathrm{q}}
\end{aligned}
$$

where $\rho_{\mathrm{q}}$ is the mean volumetric charge density which can be expressed in terms of the distribution function itself as

$$
\rho_{\mathrm{q}}(\mathbf{r}, t)=n Q(\mathbf{r}, t)=\int_{q} \int_{\mathbf{v}} q f(\mathbf{r}, t ; \mathbf{v}, q) d \mathbf{v} d q
$$

Here $Q$ is the mean or macroscopic charge. The equation set (2.5)-(2.9) is similar to the Vlasov equations for collisionless plasmas (Pitaevskii \& Lifshitz, 2012), except the fact that here a more involved collision integral which accounts for granular inelastic collisions and collisional charge exchange needs to be augmented.

\subsection{Macroscopic quantities}

In principle, once the distribution function is suitably defined, any macroscopic observable $\langle\psi\rangle(\mathbf{r}, t)$ can be expressed by taking average of its microscopic counterpart $\psi(\mathbf{v}, q)$, i.e.

$$
\langle\psi\rangle(\mathbf{r}, t)=\frac{1}{n} \int_{q} \int_{\mathbf{v}} \psi(\mathbf{v}, q) f(\mathbf{r}, t ; \mathbf{v}, q) d \mathbf{v} d q
$$


In addition to the particle number density $n$ and and the mean charge density $\rho_{\mathrm{q}}$ given in Eqs. (2.2) and (2.9), respectively, the following macroscopic quantities are useful, and are used in the thesis. The mean, or macroscopic velocity $\mathbf{V}$ is given by the relation

$$
n \mathbf{V}(\mathbf{r}, t)=\int_{q} \int_{\mathbf{v}} \mathbf{v} f(\mathbf{r}, t ; \mathbf{v}, q) d \mathbf{v} d q
$$

The granular temperature $T$, or the mean velocity fluctuations, is given by the relation

$$
\frac{3}{2} n T(\mathbf{r}, t)=\int_{q} \int_{\mathbf{v}} \frac{1}{2} m(\mathbf{v}-\mathbf{V})^{2} f(\mathbf{r}, t ; \mathbf{v}, q) d \mathbf{v} d q
$$

In addition to the mean charge $Q$, another quantity of interest will be the mean charge fluctuations $\left\langle\delta q^{2}\right\rangle$, given by

$$
n\left\langle\delta q^{2}\right\rangle(\mathbf{r}, t)=\int_{q} \int_{\mathbf{v}}(q-Q)^{2} f(\mathbf{r}, t ; \mathbf{v}, q) d \mathbf{v} d q
$$

\subsection{Collision integral}

To make the collision term Eq. 2.6 in the Boltzmann equation analytically tractable, the following simplifications are made:

(i) It is considered that the collisions between particles are instantaneous. Thus the description of the collisions in a spatial volume element $d \mathbf{r}$ is probabilistic, and the description of the events taking place within the collision time is simplified.

(ii) The particle collisions are considered to be binary. This assumption requires that the gas is sufficiently rarefied so that the particle interactions involving more than two particles can be neglected.

(iii) Before each collision, the velocities, and the charges of two colliding particles are uncorrelated.

To derive the collision integral within the above framework, the phase space volume element is first written as $d \mathbf{r} d \mathcal{V}$, where $\mathcal{V}$ are the phase space variables other than position $\mathbf{r}$ and time $t, e . g ., d \mathcal{V}=d \mathbf{v}$ for neutral granular gas and $d \mathcal{V}=d \mathbf{v} d q$ for charged granular 
gas. Thus $f(\mathbf{r}, t ; \mathcal{V}) d \mathcal{V}$ are the number of particles per unit spatial volume, irrespective of the number of variables in $\mathcal{V}$.

Let us consider two colliding particles, $i$ and $j$, having values $\mathcal{V}_{i}$ and $\mathcal{V}_{j}$ in $d \mathcal{V}_{i}$ and $d \mathcal{V}_{j}$, respectively. Due to the collision, these values will alter and both the particles will find new values $\mathcal{V}_{i}^{\prime}$ and $\mathcal{V}_{j}^{\prime}$ in $d \mathcal{V}_{i}^{\prime}$ and $d \mathcal{V}_{j}^{\prime}$ respectively. The aim is to find total number of particles $j$ colliding with particles $i$ per unit spatial volume and per unit time, say $\nu$, which lead to $\left(\mathcal{V}_{i}, \mathcal{V}_{j}\right) \rightarrow\left(\mathcal{V}_{i}^{\prime}, \mathcal{V}_{j}^{\prime}\right)$.

The number of collisions per unit time and per unit spatial volume $\nu$ will be proportional to the number of particles per unit spatial volume $f\left(\mathbf{r}, t ; \mathcal{V}_{i}\right) d \mathcal{V}_{i}$. However, there will be a probability that each of them undergoes a collision with particles $j$. This probability will be higher if the number of colliding particles $j$ per unit spatial volume, $f\left(\mathbf{r}, t ; \mathcal{V}_{j}\right) d \mathcal{V}_{j}$, is higher. Also, the larger the post-collision ranges $d \mathcal{V}_{i}^{\prime}$ and $d \mathcal{V}_{j}^{\prime}$, the larger is this probability. Collecting these arguments

$$
\nu \propto f\left(\mathbf{r}, t ; \mathcal{V}_{i}\right) d \mathcal{V}_{i} f\left(\mathbf{r}, t ; \mathcal{V}_{j}\right) d \mathcal{V}_{j} d \mathcal{V}_{i}^{\prime} d \mathcal{V}_{j}^{\prime}
$$

or

$$
\nu=p f\left(\mathbf{r}, t ; \mathcal{V}_{i}\right) d \mathcal{V}_{i} f\left(\mathbf{r}, t ; \mathcal{V}_{j}\right) d \mathcal{V}_{j} d \mathcal{V}_{i}^{\prime} d \mathcal{V}_{j}^{\prime}
$$

where the proportionality constant $p$ is determined in the following way. Each of the particles from $f\left(\mathbf{r}, t ; \mathcal{V}_{i}\right) d \mathcal{V}_{i}$ will collide with only incoming particles from $f\left(\mathbf{r}, t ; \mathcal{V}_{j}\right) d \mathcal{V}_{j}$. Or in other words the collisions will take place only if the velocities of particles $j$ relative to particles $i$, i.e. $\mathbf{v}_{j}-\mathbf{v}_{i} \equiv \mathbf{v}_{i j}$, are directed towards particles $i$ [Fig. 2.1]. If $\mathbf{n}_{i j}=\frac{\mathbf{r}_{j}-\mathbf{r}_{i}}{\left|\mathbf{r}_{j}-\mathbf{r}_{i}\right|}$ is the unit vector pointing from particle $i$ towards particle $j$ at the time of contact, then the above condition says that $-\mathbf{v}_{i j} \cdot \mathbf{n}_{i j}>0$ for collisions to take place, or in other words $\Theta\left(-\mathbf{v}_{i j} \cdot \mathbf{n}_{i j}\right)=1$. Also, if the particle $j$ has to satisfy the condition $\Theta\left(-\mathbf{v}_{i j} \cdot \mathbf{n}_{i j}\right)=1$ at the instant of the collision, then it should be at a distance $\left|\mathbf{v}_{i j}\right| d t$ from its location at the moment of contact [Fig. 2.1]. Or in the direction along $\mathbf{n}_{i j}$, it should be at a distance $\left|\mathbf{v}_{i j} \cdot \mathbf{n}_{i j}\right| d t$ from its location at the moment of contact [Fig. 2.1]. If $d \sigma$ is the differential area around $\mathbf{n}_{i j}$ on which the collisions of particles $j$ are to be counted, then the particles $j$ only within a cylinder of volume $d \sigma\left|\mathbf{v}_{i j} \cdot \mathbf{n}_{i j}\right| d t$ will collide with $i$. The number of such 


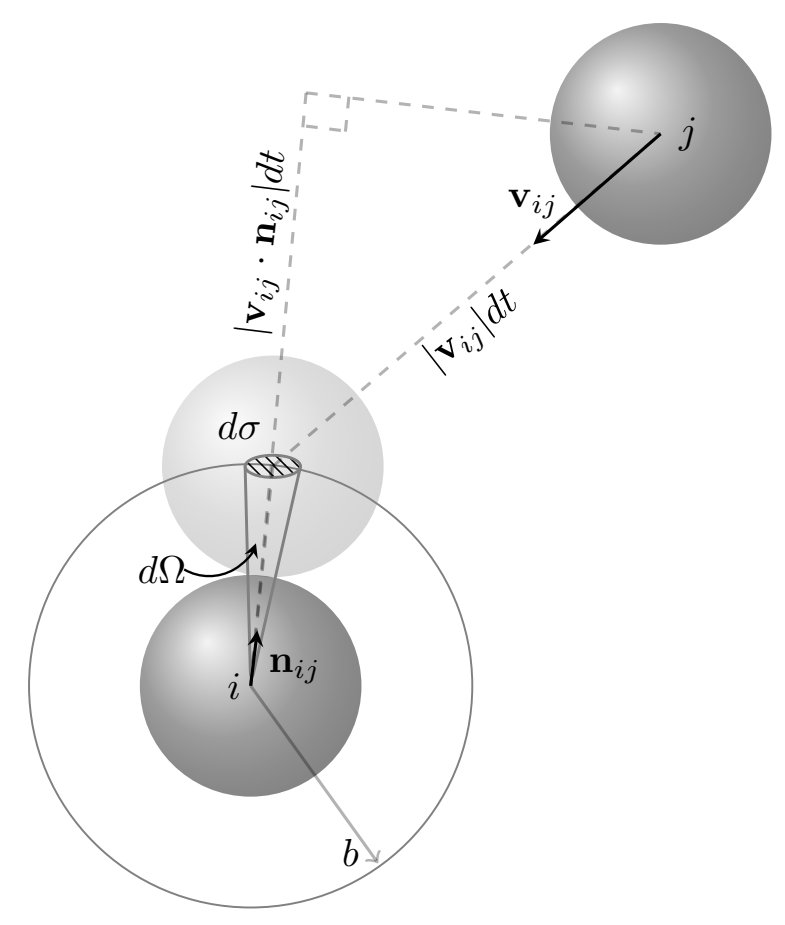

Figure 2.1: Geometrical description of a binary collision between neutral particles. A particle $j$ collides with particle $i$, within a differential time $d t$, only if the relative velocity $\mathbf{v}_{i j}$ points towards a region covered by the impact parameter $b$, and the particle $j$ is within a distance $\left|\mathbf{v}_{i j}\right| d t$ from the point of contact. The direction $\mathbf{n}_{i j}$ is the unit vector from the center of particle $i$ towards the center of particle $j$ at the instant of contact, and $\frac{d \sigma}{d \Omega}$ is the differential cross section per unit solid angle around $\mathbf{n}_{i j}$.

particles $j$ colliding with the particle $i$ will be $f\left(\mathbf{r}, t ; \mathcal{V}_{j}\right) d \mathcal{V}_{j} \Theta\left(-\mathbf{v}_{i j} \cdot \mathbf{n}_{i j}\right) d \sigma\left|\mathbf{v}_{i j} \cdot \mathbf{n}_{i j}\right| d t$. Now as there are $f\left(\mathbf{r}, t ; \mathcal{V}_{i}\right) d \mathcal{V}_{i}$ particles of type $i$ per unit spatial volume, the total number of collisions per unit spatial volume in time $d t$ will be

$$
f\left(\mathbf{r}, t ; \mathcal{V}_{i}\right) d \mathcal{V}_{i} f\left(\mathbf{r}, t ; \mathcal{V}_{j}\right) d \mathcal{V}_{j} \Theta\left(-\mathbf{v}_{i j} \cdot \mathbf{n}_{i j}\right) d \sigma\left|\mathbf{v}_{i j} \cdot \mathbf{n}_{i j}\right| d t
$$

or the number of collisions per unit time and per unit spatial volume will be

$$
\nu=f\left(\mathbf{r}, t ; \mathcal{V}_{i}\right) d \mathcal{V}_{i} f\left(\mathbf{r}, t ; \mathcal{V}_{j}\right) d \mathcal{V}_{j} \Theta\left(-\mathbf{v}_{i j} \cdot \mathbf{n}_{i j}\right) d \sigma\left|\mathbf{v}_{i j} \cdot \mathbf{n}_{i j}\right|
$$

Comparing Eqs. (2.15) and (2.17), the proportionality coefficient $p$ turns out to be

$$
p\left(\mathcal{V}_{i}^{\prime}, \mathcal{V}_{i}^{\prime} ; \mathcal{V}_{i}, \mathcal{V}_{j}\right)=\frac{\Theta\left(-\mathbf{v}_{i j} \cdot \mathbf{n}_{i j}\right) d \sigma\left|\mathbf{v}_{i j} \cdot \mathbf{n}_{i j}\right|}{d \mathcal{V}_{i}^{\prime} d \mathcal{V}_{j}^{\prime}}
$$


The number of collisions per unit time and per unit spatial volume [Eq. (2.17)] determines the number of collisions, or the number of particles that are leaving the volumes $d \mathcal{V}_{i}, d \mathcal{V}_{j}$, per unit time and per unit spatial volume. At the same time, there might be collisions which lead to increase the number of particles in the volumes $d \mathcal{V}_{i}, d \mathcal{V}_{j}$. Let us consider that these collisions take place in the ranges $d \mathcal{V}_{i}^{\prime \prime}, d \mathcal{V}_{j}^{\prime \prime}$, where the superscript " now indicates pre-collision values. With similar arguments as above, the number of such collisions per unit time and per unit spatial volume, $\nu^{+}$, will be

$$
\nu^{+}=f\left(\mathbf{r}, t ; \mathcal{V}_{i}^{\prime \prime}\right) d \mathcal{V}_{i}^{\prime \prime} f\left(\mathbf{r}, t ; \mathcal{V}_{j}^{\prime \prime}\right) d \mathcal{V}_{j}^{\prime \prime} \Theta\left(-\mathbf{v}_{i j}^{\prime \prime} \cdot \mathbf{n}_{i j}\right) d \sigma\left|\mathbf{v}_{i j}^{\prime \prime} \cdot \mathbf{n}_{i j}\right|
$$

Notice that for these so called "inverse" collisions

$$
p\left(\mathcal{V}_{i}, \mathcal{V}_{i} ; \mathcal{V}_{i}^{\prime \prime}, \mathcal{V}_{j}^{\prime \prime}\right)=\frac{\Theta\left(-\mathbf{v}_{i j}^{\prime \prime} \cdot \mathbf{n}_{i j}\right) d \sigma^{\prime \prime}\left|\mathbf{v}_{i j}^{\prime \prime} \cdot \mathbf{n}_{i j}\right|}{d \mathcal{V}_{i} d \mathcal{V}_{j}}
$$

The difference $\nu^{+}-\nu$ is the net change in the number of particles per unit spatial volume and per unit time, written as

$$
\begin{aligned}
\nu^{+}-\nu= & f\left(\mathbf{r}, t ; \mathcal{V}_{i}^{\prime \prime}\right) d \mathcal{V}_{i}^{\prime \prime} f\left(\mathbf{r}, t ; \mathcal{V}_{j}^{\prime \prime}\right) d \mathcal{V}_{j}^{\prime \prime} \Theta\left(-\mathbf{v}_{i j}^{\prime \prime} \cdot \mathbf{n}_{i j}\right) d \sigma\left|\mathbf{v}_{i j}^{\prime \prime} \cdot \mathbf{n}_{i j}\right| \\
& -f\left(\mathbf{r}, t ; \mathcal{V}_{i}\right) d \mathcal{V}_{i} f\left(\mathbf{r}, t ; \mathcal{V}_{j}\right) d \mathcal{V}_{j} \Theta\left(-\mathbf{v}_{i j} \cdot \mathbf{n}_{i j}\right) d \sigma\left|\mathbf{v}_{i j} \cdot \mathbf{n}_{i j}\right|
\end{aligned}
$$

The normal component of the pre-collision velocities $\mathbf{v}_{i j}^{\prime \prime}$ are related to their post collision values $\mathbf{v}_{i j}$ via the coefficient of restitution as

$$
\mathbf{v}_{i j}^{\prime \prime} \cdot \mathbf{n}_{i j}=-\frac{1}{\epsilon} \mathbf{v}_{i j} \cdot \mathbf{n}_{i j}
$$

where $0 \leq \epsilon \leq 1$ is the coefficient of restitution. For further simplification, the volume elements $d \mathcal{V}_{i}^{\prime \prime}, d \mathcal{V}_{j}^{\prime \prime}$ are to be transformed to $d \mathcal{V}_{i}, d \mathcal{V}_{j}$ using

$$
d \mathcal{V}_{i}^{\prime \prime} d \mathcal{V}_{j}^{\prime \prime}=\frac{\partial\left(\mathcal{V}_{i}^{\prime \prime}, \mathcal{V}_{j}^{\prime \prime}\right)}{\partial\left(\mathcal{V}_{i}, \mathcal{V}_{j}\right)} d \mathcal{V}_{i} d \mathcal{V}_{j}
$$

where $\frac{\partial\left(\mathcal{V}_{i}^{\prime \prime}, \mathcal{V}_{j}^{\prime \prime}\right)}{\partial\left(\mathcal{V}_{i}, \mathcal{V}_{j}\right)}$ is the determinant of the Jacobian of the transformation.

Using Eqs. (2.22) and (2.23) in Eq. (2.21), dividing by $d \mathcal{V}_{i}$, and integrating over variables $\mathcal{V}_{j}$ and all possible directions $\mathbf{n}_{i j}$, the following form of the collision integral is 
obtained

$$
\begin{aligned}
I^{\text {coll }}=\frac{\partial}{\partial t} f\left(\mathbf{r}, t ; \mathcal{V}_{i}\right)= & \int_{\mathcal{V}_{j}} \int_{\mathbf{n}_{i j}}\left[\chi f\left(\mathbf{r}, t ; \mathcal{V}_{i}^{\prime \prime}\right) f\left(\mathbf{r}, t ; \mathcal{V}_{j}^{\prime \prime}\right)-f\left(\mathbf{r}, t ; \mathcal{V}_{i}\right) f\left(\mathbf{r}, t ; \mathcal{V}_{j}\right)\right] \\
& \times \Theta\left(-\mathbf{v}_{i j} \cdot \mathbf{n}_{i j}\right)\left|\mathbf{v}_{i j} \cdot \mathbf{n}_{i j}\right| d \mathcal{V}_{j} d \sigma
\end{aligned}
$$

where

$$
\chi=\frac{\left|\mathbf{v}_{i j}^{\prime \prime} \cdot \mathbf{n}_{i j}\right|}{\left|\mathbf{v}_{i j} \cdot \mathbf{n}_{i j}\right|} \frac{\partial\left(\mathcal{V}_{i}^{\prime \prime}, \mathcal{V}_{j}^{\prime \prime}\right)}{\partial\left(\mathcal{V}_{i}, \mathcal{V}_{j}\right)}
$$

Note that according to the definition, the phase space variables $\mathcal{V}$ includes velocity, as well other variables of interest. For example for a neutral granular gas $\mathcal{V}=\mathbf{v}$ only, while for granular gas of charged particles, $\mathcal{V}=\{\mathbf{v}, q\}$. Also $d \sigma \equiv \frac{d \sigma}{d \Omega} d \Omega \equiv \frac{d \sigma}{d \Omega} d \mathbf{n}_{i j}$, with $\frac{d \sigma}{d \Omega}$ being the differential cross section per unit solid angle around $\mathbf{n}_{i j}$ [Fig. 2.1]. Thus for specifying the above collision integral for a given set of $\mathcal{V}$, the collision rule for each of the component variables in $\mathcal{V}$ should be specified. Below, the case of neutral granular gas is first discussed, where $\mathcal{V}=\mathbf{v}$.

\subsubsection{Collision integral for a neutral granular gas}

For a neutral granular gas $\mathcal{V}=\mathbf{v}$, where the Jacobian simplifies to $\frac{\partial\left(\mathcal{V}_{i}^{\prime \prime}, \mathcal{V}_{j}^{\prime \prime}\right)}{\partial\left(\mathcal{V}_{i}, \mathcal{V}_{j}\right)}=\frac{\partial\left(\mathbf{v}_{i}^{\prime \prime}, \mathbf{v}_{j}^{\prime \prime}\right)}{\partial\left(\mathbf{v}_{i}, \mathbf{v}_{j}\right)}$, and can be found by specifying the relation between pre and post collision velocities. The relation between the normal component of relative velocities is given in Eq. (2.22). In this thesis, the frictional forces are ignored and thus the tangential component of relative velocities remain unchanged, i.e.

$$
\mathbf{v}_{i j}-\left(\mathbf{v}_{i j} \cdot \mathbf{n}_{i j}\right) \mathbf{n}_{i j}=\mathbf{v}_{i j}^{\prime \prime}-\left(\mathbf{v}_{i j}^{\prime \prime} \cdot \mathbf{n}_{i j}\right) \mathbf{n}_{i j}
$$

Combining Eqs. (2.22) and (2.26) provides

$$
\mathbf{v}_{i j}=\mathbf{v}_{i j}^{\prime \prime}-(1+\epsilon)\left(\mathbf{v}_{i j}^{\prime \prime} \cdot \mathbf{n}_{i j}\right) \mathbf{n}_{i j}
$$


Using the momentum balance

$$
m_{0, i} \mathbf{v}_{i}^{\prime \prime}+m_{0, j} \mathbf{v}_{j}^{\prime \prime}=m_{0, i} \mathbf{v}_{i}+m_{0, j} \mathbf{v}_{j}
$$

in Eq. (2.27), the post-collision velocities in terms of pre-collision velocities are written as

$$
\begin{aligned}
& \mathbf{v}_{i}=\mathbf{v}_{i}^{\prime \prime}+\frac{1+\epsilon}{2}\left(\mathbf{v}_{i j}^{\prime \prime} \cdot \mathbf{n}_{i j}\right) \mathbf{n}_{i j} \\
& \mathbf{v}_{j}=\mathbf{v}_{j}^{\prime \prime}-\frac{1+\epsilon}{2}\left(\mathbf{v}_{i j}^{\prime \prime} \cdot \mathbf{n}_{i j}\right) \mathbf{n}_{i j}
\end{aligned}
$$

where it is assumed that $m_{0, i}=m_{0, j}$. Alternatively, the pre-collision velocities in terms of post-collision velocities, using $\mathbf{v}_{i j}^{\prime \prime} \cdot \mathbf{n}_{i j}=-\frac{1}{\epsilon} \mathbf{v}_{i j} \cdot \mathbf{n}_{i j}$, are written as

$$
\begin{aligned}
& \mathbf{v}_{i}^{\prime \prime}=\mathbf{v}_{i}+\frac{1+\epsilon}{2 \epsilon}\left(\mathbf{v}_{i j} \cdot \mathbf{n}_{i j}\right) \mathbf{n}_{i j} \\
& \mathbf{v}_{j}^{\prime \prime}=\mathbf{v}_{j}-\frac{1+\epsilon}{2 \epsilon}\left(\mathbf{v}_{i j} \cdot \mathbf{n}_{i j}\right) \mathbf{n}_{i j} .
\end{aligned}
$$

These relations are then used to obtain the transformation $d \mathbf{v}_{i}^{\prime \prime} d \mathbf{v}_{j}^{\prime \prime}=\frac{\partial\left(\mathbf{v}_{i}^{\prime \prime}, \mathbf{v}_{j}^{\prime \prime}\right)}{\partial\left(\mathbf{v}_{i}, \mathbf{v}_{j}\right)} d \mathbf{v}_{i} d \mathbf{v}_{j}$. For example, considering a collision where $\mathbf{n}_{i j}=(0,0,1)$, the Eqs. (2.31) read as

$$
\begin{gathered}
v_{i, x}^{\prime \prime}=v_{i, x}, v_{i, y}^{\prime \prime}=v_{i, y}, v_{i, z}^{\prime \prime}=v_{i, z}+\frac{1+\epsilon}{2 \epsilon}\left(v_{j, z}-v_{i, z}\right), \\
v_{j, x}^{\prime \prime}=v_{j, x}, v_{j, y}^{\prime \prime}=v_{j, y}, v_{j, z}^{\prime \prime}=v_{j, z}-\frac{1+\epsilon}{2 \epsilon}\left(v_{j, z}-v_{i, z}\right) .
\end{gathered}
$$

Now considering the case of a constant $\epsilon$, the Jacobian of transformation reads

$$
\frac{\partial\left(\mathbf{v}_{i}^{\prime \prime}, \mathbf{v}_{j}^{\prime \prime}\right)}{\partial\left(\mathbf{v}_{i}, \mathbf{v}_{j}\right)}=\frac{1}{\epsilon}
$$

Incorporating this into the definition [Eq. (2.24)], the collision integral for a neutral granular gas can be written as

$$
\begin{aligned}
I^{\text {coll }}= & \int_{\mathbf{v}_{j}} \int_{\mathbf{n}_{i j}}\left[\chi f\left(\mathbf{r}, \mathbf{v}_{i}^{\prime \prime} ; t\right) f\left(\mathbf{r}, \mathbf{v}_{j}^{\prime \prime} ; t\right)-f\left(\mathbf{r}, \mathbf{v}_{i} ; t\right) f\left(\mathbf{r}, \mathbf{v}_{j} ; t\right)\right] \\
& \times \Theta\left(-\mathbf{v}_{i j} \cdot \mathbf{n}_{i j}\right)\left|\mathbf{v}_{i j} \cdot \mathbf{n}_{i j}\right| d \mathbf{v}_{j} d \sigma
\end{aligned}
$$


Using Eqs. (2.22) and (2.33) in equation (2.25), the value of $\chi$ for a granular gas with constant $\epsilon$ turns out to be $\frac{1}{\epsilon^{2}}$. For a granular gas with $\epsilon=\epsilon\left(\left|\mathbf{v}_{i j} \cdot \mathbf{n}_{i j}\right|\right)$, the transformation $d \mathbf{v}_{i}^{\prime \prime} d \mathbf{v}_{j}^{\prime \prime} \rightarrow d \mathbf{v}_{i} d \mathbf{v}_{j}$ is more involved. For example in this case the expression for $\chi$, under certain assumptions, is derived by Brilliantov \& Pöschel (2010), which reads as

$$
\chi=\frac{1}{\epsilon\left(\left|\mathbf{v}_{i j} \cdot \mathbf{n}_{i j}\right|\right)}\left[1+c_{0} \frac{6}{5}\left|\mathbf{v}_{i j} \cdot \mathbf{n}_{i j}\right|^{1 / 5}+\ldots\right],
$$

where $c_{0}$ is a material constant.

\subsubsection{Modified collision integral for a charged granular gas}

For a granular gas of charged particles, $\mathcal{V}=\{\mathbf{v}, q\}$. Thus, the Jacobian of transformation $d q_{i}^{\prime \prime} d q_{j}^{\prime \prime} \rightarrow d q_{i} d q_{j}$ is also required in addition to the transformation $d \mathbf{v}_{i}^{\prime \prime} d \mathbf{v}_{j}^{\prime \prime} \rightarrow d \mathbf{v}_{i} d \mathbf{v}_{j}$. The expression for $\chi$ in this case reads

$$
\chi=\frac{\left|\mathbf{v}_{i j}^{\prime \prime} \cdot \mathbf{n}_{i j}\right|}{\left|\mathbf{v}_{i j} \cdot \mathbf{n}_{i j}\right|} \frac{\partial\left(\mathbf{v}_{i}^{\prime \prime}, \mathbf{v}_{j}^{\prime \prime}\right)}{\partial\left(\mathbf{v}_{i}, \mathbf{v}_{j}\right)} \frac{\partial\left(q_{i}^{\prime \prime}, q_{j}^{\prime \prime}\right)}{\partial\left(q_{i}, q_{j}\right)}
$$

In relation to this point, several intricacies need to be addressed. First, the grains in a granular gas may be of conducting or dielectric materials. However, in the context of protoplanetary dust evolution, it is assumed that the grains are of dielectric nature. Second, the grains have finite size and thus it is possible that the charge is distributed on the grain surface in a complex manner. Third, the charge transfer between two colliding dielectric grains, in itself, is not a well understood phenomenon. Fourth, the relative collision velocity might correlate with the grain surface charges altering the collision rule significantly in an intricate manner. Therefore in practice, the transformation $d q_{i}^{\prime \prime} d q_{j}^{\prime \prime} \rightarrow$ $d q_{i} d q_{j}$ is quite involved.

For analytical tractability, the following simplifying assumptions are made. The charges on the grains influence the impact parameter, or the differential cross section (say $d \sigma_{\mathrm{q}}$ ), however, the restitution rule for viscoelastic particles [Eq. (2.31)] is assumed to remain unaltered during the course of a collision. In other words, the Jacobian $\frac{\partial\left(\mathbf{v}_{i}^{\prime \prime}, \mathbf{v}_{j}^{\prime \prime}\right)}{\partial\left(\mathbf{v}_{i}, \mathbf{v}_{j}\right)}$ is considered to keep its form similar to the neutral viscoelastic granular gas. The grains are considered to carry charge monopoles at their COM, and the values of the charges alter only during the course of a collision. As a simple specific example, consider that in 
addition to the charge conservation during collisions

$$
q_{j}^{\prime \prime}+q_{i}^{\prime \prime}=q_{j}+q_{i}
$$

the ratio of pre to post collision relative charges is defined as

$$
\kappa=\frac{q_{j}-q_{i}}{q_{j}^{\prime \prime}-q_{i}^{\prime \prime}}
$$

The above two equations lead to the collision rule

$$
\begin{aligned}
& q_{i}^{\prime \prime}=\frac{q_{j}+q_{i}}{2}-\frac{1}{2 \kappa}\left(q_{j}-q_{i}\right), \\
& q_{j}^{\prime \prime}=\frac{q_{j}+q_{i}}{2}+\frac{1}{2 \kappa}\left(q_{j}-q_{i}\right) .
\end{aligned}
$$

Using the above equation, and considering a granular gas with constant $\kappa$, the determinant of the Jacobian of transformation reads

$$
\frac{\partial\left(q_{i}^{\prime \prime}, q_{j}^{\prime \prime}\right)}{\partial\left(q_{i}, q_{j}\right)}=\frac{1}{2}\left|\begin{array}{ll}
\left(1+\frac{1}{\kappa}\right) & \left(1-\frac{1}{\kappa}\right) \\
\left(1-\frac{1}{\kappa}\right) & \left(1+\frac{1}{\kappa}\right)
\end{array}\right|=\frac{2}{\kappa} .
$$

Thus for a granular gas with constant $\epsilon$ and constant $\kappa$,

$$
\chi=\frac{2}{\epsilon^{2} \kappa}
$$

while for a granular gas with $\epsilon=\epsilon\left(\left|\mathbf{v}_{i j} \cdot \mathbf{n}_{i j}\right|\right)$ and constant $\kappa$,

$$
\chi=\frac{2}{\kappa} \frac{1}{\epsilon\left(\left|\mathbf{v}_{i j} \cdot \mathbf{n}_{i j}\right|\right)}\left[1+c_{0} \frac{6}{5}\left|\mathbf{v}_{i j} \cdot \mathbf{n}_{i j}\right|^{1 / 5}+\ldots\right] .
$$

In practice, $\kappa$ is expected not to be constant. Therefore, the notation $\chi$ is used as a general variable in the collision integral. While taking moments of the collision integral to determine the time rate of change of macroscopic quantities, it can be shown that the actual expression for $\chi$ is not required, and the charge exchange rule can be used directly. This helps in incorporating the present knowledge (empirical or analytical) of the charge exchange mechanism into the kinetic description. This point is further discussed in chapter 5, where the time evolutions of the macroscopic quantities are derived. 


\subsection{Mean-field description of aggregation: Smolu- chowski coagulation equation}

A sophisticated collision dynamics is considered in the kinetic theory above. In the following, an alternative mean-field approach, also used in the thesis, is described to determine the growth rate of aggregates in a charged granular gas.

Consider an aggregation process where the motion of particles, and their mutual interactions, are spatially isotropic. The particles are considered to aggregate, or stick together, with some probability $s$ upon collisions. The collision time, i.e. the time taken by the particles during the course of a collision, is considered much smaller than the time taken by particles to travel the mean free path $\bar{r}$. The smallest particles during the aggregation process, or monomers, have diameter $d_{0}$ and mass $m_{0}$, and are initially monodispersed. The variable of interest is the mass $m$ of an aggregate at a given time $t$.

Let us consider a mass distribution function $f(m, t)$, normalized such that $f(m, t) d m$ provides the number of particles/aggregates per unit spatial volume (or the particle/aggregate number density), which have mass in the range $d m$ around $m$, at time $t$. The number of collisions which lead to aggregation of particles, having masses in the range $d m_{i}$ and $d m_{j}$, per unit time and per unit spatial volume, will be

$$
\nu^{\text {agg, },-}=s_{i j} v_{i j} \sigma_{i j} f\left(m_{i}, t\right) d m_{i} f\left(m_{j}, t\right) d m_{j},
$$

where, $v_{i j}$ is the relative collision speed and $\sigma_{i j}$ is the total collision cross section between the particles/aggregates $i$ and $j . s_{i j}$ is the probability (called sticking probability) that a collision between particles $i$ and $j$ will lead to aggregation. The superscript "-" is used to emphasize that the particles, after aggregation, will leave the ranges $d m_{i}$ and $d m_{j}$.

Similarly, there will be collisions, where the particle masses will enter into the range $d m_{i}$ around $m_{i}$. If $m_{j}^{\prime \prime}$ are the pre-collision masses, which have to enter the mass range $d m_{i}$, they must collide with particles having masses $m_{k}^{\prime \prime}=\left(m_{i}-m_{j}^{\prime \prime}\right)$. The number of collisions which satisfy this condition, per unit time and per unit spatial volume, will be

$$
\nu^{\mathrm{agg},+}=s_{i j}^{\prime \prime} v_{i j}^{\prime \prime} \sigma_{i j}^{\prime \prime} f\left(m_{i}-m_{j}^{\prime \prime}, t\right) d m_{k}^{\prime \prime} f\left(m_{j}^{\prime \prime}, t\right) d m_{j}^{\prime \prime}
$$


Subtracting Eq. (2.43) from Eq. (2.44), dividing by $d m_{i}$, and integrating over appropriate ranges of masses, one obtains the time rate of change of $f\left(m_{i}, t\right)$

$$
\begin{aligned}
\frac{\partial}{\partial t} f\left(m_{i}, t\right)= & \int_{0}^{m_{i} / 2} s_{i j}^{\prime \prime} v_{i j}^{\prime \prime} \sigma_{i j}^{\prime \prime} f\left(m_{i}-m_{j}^{\prime \prime}, t\right) \frac{d m_{k}^{\prime \prime}}{d m_{i}} f\left(m_{j}^{\prime \prime}, t\right) d m_{j}^{\prime \prime} \\
& -\int_{0}^{\infty} s_{i j} v_{i j} \sigma_{i j} f\left(m_{i}, t\right) f\left(m_{j}, t\right) d m_{j}
\end{aligned}
$$

The above equation can be considered as a general form of the Smoluchowski coagulation equation, excluding the fragmentation events. The integration limits in the gain term (first term on the RHS) are such that $(i)$ they satisfy $m_{k}^{\prime \prime}+m_{j}^{\prime \prime}=\left(m_{i}-m_{j}^{\prime \prime}\right)+m_{j}^{\prime \prime}=m_{i}$, and (ii) the particles are not counted twice. Assuming that the differential mass elements $d m_{i}=d m_{j}^{\prime \prime}=d m_{k}^{\prime \prime}$, Eq. (2.45) can be simplified to

$$
\begin{aligned}
\frac{\partial}{\partial t} f\left(m_{i}, t\right)= & \int_{0}^{m_{i} / 2} K_{i-j, j} f\left(m_{i}-m_{j}, t\right) f\left(m_{j}, t\right) d m_{j} \\
& -\int_{0}^{\infty} K_{i, j} f\left(m_{i}, t\right) f\left(m_{j}, t\right) d m_{j}
\end{aligned}
$$

Here the quantity

$$
K_{i, j} \equiv s_{i j} v_{i j} \sigma_{i j}
$$

in the above integro-differential equation is termed as the collision kernel, or the reaction kernel. The macroscopic number density $n(t)$ of aggregates at any given time $t$ is given by

$$
\int_{0}^{\infty} f\left(m_{i}, t\right) d m_{i}=n(t)
$$

Also, the following normalization states that at any time $t$, the total mass is conserved by the Smoluchowski equation

$$
\int_{0}^{\infty} m_{i} f\left(m_{i}, t\right) d m_{i}=\text { constant }
$$

The following points about the Smoluchowski equation need to be emphasized. The spatial information of aggregates is not accounted for, and the equation, in principle, applies 
to homogeneously mixed systems. Also, only pair collisions are considered which makes it useful for dilute particle systems. It is also assumed that the particle interactions, and motions, are spatially isotropic. There are no mass sources, or sinks, of particles. The information about aggregate morphology, relative motion, and sticking probability can be incorporated in the Smoluchowski's equation via the reaction kernel [Eq. (2.47)].

The mean field approach described above is used in chapter 4 to determine the aggregation rates, for neutral, as well as charged granular gases.

If initially, all the grains/monomers have identical mass $m_{0}$, then the mass of an aggregate $i$ will be simply $m_{i}=m_{0} N_{i}$, where $N_{i}$ is now the number of monomers in aggregate $i$. In this case, the integrals in Eq. (2.46) can be replaced by discrete sums, as

$$
\frac{\partial}{\partial t} f\left(N_{i}, t\right)=\frac{1}{2} \sum_{j=1}^{i-1} K_{i-j, j} f\left(N_{i}-N_{j}, t\right) f\left(N_{j}, t\right)-\sum_{j=1}^{\infty} K_{i, j} f\left(N_{i}, t\right) f\left(N_{j}, t\right) .
$$

In the present thesis, the smallest entities, i.e, monomers are considered monodispersed, and thus the discrete form of the Smoluchowski equation is used. The modification in the aggregation kernel $K_{i, j}$, however, is needed. An appropriate treatment of $K_{i, j}$ is developed in chapter 4 with the help of MD simulation results. 


\section{Chapter 3}

\section{Granular molecular dynamics}

\subsection{Equation of motion}

One of the important aspects in the process of planetary dust aggregation is that the nature of interactions between grains, and their motion, change as one moves from grain sizes as small as few nm to as large as meter-sized bodies. The Brownian motion and surface forces are important for grains in the size range of $\mathrm{nm}-\mu \mathrm{m}$. The aggregation processes in this regime of grain sizes have been the subject of several studies (Ivlev et al. , 2002; Blum, 2006; Castellanos, 2005). For bodies whose size is comparable to or larger than a few meters, the gravitational interaction takes over. As argued in section 1.3, the present work is focused on the grain size regime $d_{0} \sim \mathrm{mm}-\mathrm{cm}$, where neither the surface interactions, such as Van der Waals forces, nor the gravitational forces are of primary importance. Mainly the effect of electrostatic interactions originating due to the collisional electrification of the grains is investigated. The grains are athermal (i.e. they are not in Brownian motion). Their shape is assumed to be spherical. The short-range forces between grains are considered purely due to mechanical contact. The contact forces can be model specific, and their treatment is separately described in the next section.

For the translation of and the rotation about the COM of a grain $i$, Newton's equation of motion is written as

$$
\begin{aligned}
m_{i} \frac{d \mathbf{v}_{i}}{d t} & =\mathbf{F}_{i}^{\text {short }}+\mathbf{F}_{i}^{\text {long }}, \\
M_{i} \frac{d \boldsymbol{\omega}_{i}}{d t} & =\mathbf{T}_{i},
\end{aligned}
$$


where $m_{i}$ is the grain mass, $\mathbf{v}_{i}$ is the grain COM velocity, $M_{i}$ is the moment of inertia about the COM, and $\boldsymbol{\omega}_{i}$ is the angular velocity of the grain. The terms $\mathbf{F}_{i}^{\text {short }}, \mathbf{F}_{i}^{\text {long }}$ and $\mathbf{T}_{i}$ are the net short-range force, long-range-force and the torque on the grain $i$, respectively, while $t$ denotes time. Due to contact or collisional electrification, the equation for charge $q_{i}$ on a grain $i$ is written as

$$
\frac{d q_{i}}{d t}=I_{i}
$$

where $I_{i}$ are the currents acting on the grain $i$. These charge currents are introduced in sections 3.5, 3.8, 3.9, and 3.10. In this work, the grain surface is assumed to be smooth and the tangential contact forces are neglected. In practice, finite size grains might have complex, time-dependent, charge distributions on their surface and might have contributions to the rotational motion as well. Oblique collisions might also result in angular momentum exchange between grains. The above scenarios are neglected in the present work (i.e. $\mathbf{T}_{i}=0$ ), and only the translational motion of the grains is considered.

Treatment of the short and long-range forces $\mathbf{F}_{i}^{\text {short }}$ and $\mathbf{F}_{i}^{\text {long }}$ is presented in section 3.2, 3.3 and 3.4. The charge currents $I_{i}$ are introduced in section 3.5, and then the rescaling of the entire system of equations is presented in section 3.6. Numerical integration schemes, viscous friction and stochastic treatment of $I_{i}$ are discussed thereafter.

\subsection{Contact forces}

The net force $\mathbf{F}_{i}$ on a grain is the resultant of the short-range (normal) and long-range (central) forces, i.e.

$$
\mathbf{F}_{i}=\mathbf{F}_{i}^{\text {short }}+\mathbf{F}_{i}^{\text {long }}
$$

The net short-range normal force $\mathbf{F}_{i}^{\text {short }}$ on a grain $i$ is considered equal to the pairwise sum of the forces due to grains $j$, which come into mechanical contact with grain $i$, and written as

$$
\mathbf{F}_{i}^{\text {short }}=\sum_{j} \mathbf{F}_{i j}^{\text {short }} \Theta\left(d_{0}-r_{i j}\right)
$$


Here

$$
r_{i j} \equiv\left|\mathbf{r}_{i j}\right| \equiv\left|\mathbf{r}_{j}-\mathbf{r}_{i}\right|
$$

is the magnitude of the distance vector from the center of grain $i$ to the center of grain $j$. The grain diameter is denoted by $d_{0}$. Thus, a collision or mechanical contact between two particles is detected when

$$
d_{0}-r_{i j}=\xi_{i j}>0
$$

Here $\xi_{i j}$ might be understood as mutual compression between colliding pair, which changes with time during the course of a collision [Fig. 3.1]. The following two models for the computation of $\mathbf{F}_{i j}^{\text {short }}$ are considered in this work:

(i) Linear spring-dashpot model, which corresponds to a constant coefficient of restitution, and

(ii) Viscoelastic force model, which corresponds to a coefficient of restitution which depends on the relative collision velocity.

The coefficient of restitution, $\epsilon$, is defined in Fig. 3.1. In the linear spring-dashpot model, the normal contact force $\mathbf{F}_{i j}^{\text {short }}$ is written as

$$
\mathbf{F}_{i j}^{\mathrm{short}}=-\left[A \xi_{i j}+B \frac{d \xi_{i j}}{d t}\right] \mathbf{n}_{i j}
$$

where $A$ and $B$ are the elastic and dissipative constants, and

$$
\frac{d \xi_{i j}}{d t} \equiv \dot{\xi}_{i j}=-\frac{d}{d t}\left|\mathbf{r}_{i j}(t)\right|=-\mathbf{v}_{i j} \cdot \mathbf{n}_{i j}
$$

The normal vector pointing from the center of grain $i$ towards the center of grain $j$ is defined as [Fig. 3.1]

$$
\mathbf{n}_{i j} \equiv \frac{\mathbf{r}_{j}-\mathbf{r}_{i}}{\left|\mathbf{r}_{j}-\mathbf{r}_{i}\right|} \equiv \frac{\mathbf{r}_{i j}}{\left|\mathbf{r}_{i j}\right|}
$$

The first term in Eq. (3.6) is an elastic contact force which increases linearly with the mutual particle compression $\xi_{i j}$, and acts on the particle $i$ in the direction opposite to $\mathbf{n}_{i j}$ [Fig. 3.1]. The second term mimics the viscous damping force during the collision. In the decompression phase during a collision, $\frac{d \xi_{i j}}{d t}<0$, and it might happen that $A \xi_{i j}+$ 

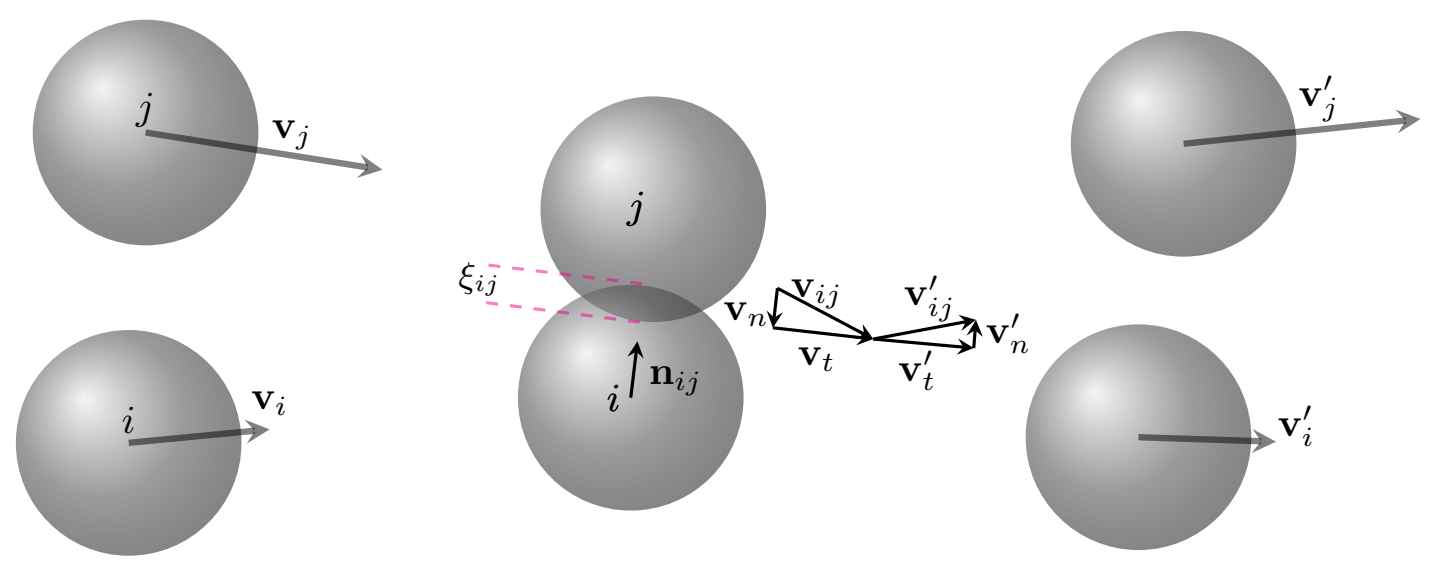

Figure 3.1: The relative velocity just before the collision between two grains $i$ and $j$ is $\mathbf{v}_{i j}=\mathbf{v}_{j}-\mathbf{v}_{i}$, and just after the collision is $\mathbf{v}_{i j}^{\prime}=\mathbf{v}_{j}^{\prime}-\mathbf{v}_{i}^{\prime}$. The normal collision velocity is $\mathbf{v}_{n}=\left(\mathbf{v}_{i j} \cdot \mathbf{n}_{i j}\right) \mathbf{n}_{i j}$ where $\mathbf{n}_{i j}=\frac{\mathbf{r}_{j}-\mathbf{r}_{i}}{\left|\mathbf{r}_{j}-\mathbf{r}_{i}\right|}=\frac{\mathbf{r}_{i j}}{\left|\mathbf{r}_{i j}\right|}$ is a unit vector pointing from the center of particle $i$ towards the center of particle $j$. The normal collision velocity just after the collision is related to the normal collision velocity just before the collision as $\mathbf{v}_{n}^{\prime}=-\epsilon \mathbf{v}_{n}$ where $0 \leq \epsilon \leq 1$ is the coefficient of normal restitution. Similarly the tangential collision velocity just after the collision is related to the tangential collision velocity just before the collision as $\mathbf{v}_{t}^{\prime}=\epsilon_{t} \mathbf{v}_{t}$ where $\epsilon_{t}$ is the coefficient of tangential restitution. In the present study $\epsilon_{t}=1$. During the course of the collision, the mutual deformation of the particles is denoted as $\xi_{i j}(t)=d_{0}-\left|\mathbf{r}_{i j}(t)\right|$.

$B \dot{\xi_{i j}}<0$. In the simulations, this might lead to a net nonphysical attraction between particles during the decompression phase of a collision. To avoid this scenario, Eq. (3.6) is implemented in the following modified form

$$
\mathbf{F}_{i j}^{\text {short }}=-\max \left[A \xi_{i j}+B \frac{d \xi_{i j}}{d t}, 0\right] \mathbf{n}_{i j}
$$

For viscoelastic grains, more than one force models have been proposed. We adopt the viscoelastic force model

$$
\mathbf{F}_{i j}^{\text {short }}=-\max \left[E \xi_{i j}^{3 / 2}+D \xi_{i j}^{1 / 2} \frac{d \xi_{i j}}{d t}, 0\right] \mathbf{n}_{i j}
$$

While the first term in this model was originally derived by Hertz (Hertz, 1882), the dissipative part was augmented in the model later (Kuwabara \& Kono, 1987; Brilliantov et al. , 1996; Pöschel \& Schwager, 2005). The elastic and dissipative constants, $E$ and $D$, in the model are further related to material properties (Young's modulus $Y$ and Poissons 
ratio $\nu_{\mathrm{P}}$ ) and the effective radius $R^{\mathrm{eff}} \equiv\left(\frac{1}{R_{i}}+\frac{1}{R_{j}}\right)^{-1}$ of the colliding pair, as

$$
\begin{aligned}
& E=\frac{2 Y \sqrt{R^{\mathrm{eff}}}}{3\left(1-\nu_{\mathrm{P}}^{2}\right)}, \\
& D=\gamma E .
\end{aligned}
$$

The constant $\gamma$ is a function of material viscosity (Brilliantov et al. , 1996). The dissipative force in Eq. (3.10) takes into account a coefficient of restitution $\epsilon$, which depends upon the relative collision velocity (Pöschel \& Schwager, 2005).

\subsection{Long-range forces: Ewald summation}

The net long-range force $\mathbf{F}_{i}^{\text {long }}$ on grain $i$ due to the monopole electrostatic interactions with all the other grains $k$ is the pairwise sum

$$
\mathbf{F}_{i}^{\text {long }}=-\sum_{k, k \neq i} k_{e} q_{i} \frac{q_{k}}{r_{i k}^{3}} \mathbf{r}_{i k}
$$

where $\mathbf{r}_{i k} \equiv \mathbf{r}_{k}-\mathbf{r}_{i}$ is the distance vector pointing from the center of grain $i$ towards the center of the grain $k$, and $q$ are the electric charges. The constant $k_{e} \equiv \frac{1}{4 \pi \varepsilon}$ is the Coulombs's constant, with $\varepsilon$ being the permittivity of the medium surrounding the grains. For grains in vacuum, $k_{e}=8.98 \times 10^{9} \mathrm{Nm}^{2} \mathrm{C}^{-2}$. For a setup of periodic boundary conditions, the above sum in the simulations is to be modified into the following form

$$
\mathbf{F}_{i}^{\text {long }}=-\sum_{\mathbf{b}} \sum_{k}^{k \neq i \text { if } \mathbf{b}=\mathbf{0}} k_{e} q_{i} \frac{q_{k}}{\left|\mathbf{r}_{i k}+\mathbf{b} L\right|^{3}}\left(\mathbf{r}_{i k}+\mathbf{b} L\right)
$$

where now the additional sum is over a set of periodic replicas of the original system of linear size $L$. This is denoted by a vector of integers, $\mathbf{b}=\left(b_{x}, b_{y}, b_{z}\right) \in \mathbb{Z}^{3}$. When the above sum is performed for each of the $N_{\text {mon }}$ grains in the original image of the system, the computational time increases as $\mathcal{O}\left(N_{\text {mon }}^{2}\right)$, which is computationally intractable when $N_{\text {mon }}$ becomes large. One of the standard remedy to this problem is the Ewald summation, which is implemented and parallelized in the simulations in this thesis. Next, the details of the Ewald summation are briefly described. 
The electrostatic force on a grain $i$ carrying charge $q_{i}$ in Eq. (3.14) can be rewritten as

$$
\mathbf{F}_{i}^{\text {long }}=-q_{i} \frac{\partial}{\partial \mathbf{r}_{i}} U\left(\mathbf{r}_{i}\right)
$$

where $U\left(\mathbf{r}_{i}\right)$ is the electrostatic (central) potential at the location of particle $i$ due to the surrounding charges $k$, written as

$$
U\left(\mathbf{r}_{i}\right)=k_{e} \sum_{\mathbf{b}} \sum_{k}^{k \neq i \text { if } \mathbf{b}=\mathbf{0}} \frac{q_{k}}{\left|\mathbf{r}_{i k}+\mathbf{b} L\right|}
$$

Consider the original image of the system $\mathbf{b}=\mathbf{0}$. For each particle $i$, there are $N_{\text {mon }}-1$ terms in the above sum, where $N_{\text {mon }}$ is the total number of monomers/particles in the original image. When the potential is computed at the location of each of the $N_{\text {mon }}$ particles, the total number of terms to be dealt with is $N_{\text {mon }}\left(N_{\text {mon }}-1\right)$. The total number of terms can be reduced to $\frac{1}{2} N_{\text {mon }}\left(N_{\text {mon }}-1\right)$ if Newton's third law is exploited. However, as $N_{\text {mon }} \rightarrow \infty$, the computational operations still increase with $\mathcal{O}\left(N_{\text {mon }}^{2}\right)$. This is one of the challenging aspect in $N$-body problems involving long range interactions (another example is self-gravitation).

In general, the potentials decaying slower than $\sim r_{i k}^{-a}$, where $a$ is the number of spatial dimensions in the problem, have the characteristic that their range exceeds the half of the linear size of the system, and the sum over the potentials due to only nearest neighbors is not enough (Allen \& Tildesley, 2017). The potential in Eq. (3.16) drops as $\sim r_{i k}^{-1}$ with the interparticle distance $r_{i k}$. When one has to adopt a periodic system to eliminate or minimize the finite-size/boundary surface-effects, the computation becomes even more involved, in addition to the $\mathcal{O}\left(N_{\text {mon }}^{2}\right)$ problem. This is due to the reason that the direct sum, in this case, is conditionally convergent with an increasing number of periodic replicas $\mathbf{b}$.

An established remedy to the above problem is the Ewald-summation method, in which the summation series (3.16) is split into a sum of two series, which converge rapidly with increasing number of periodic replicas. The basic idea is to split the sum of $r_{i k}^{-1}$ po- 
tentials simply as

$$
\begin{aligned}
U\left(\mathbf{r}_{i}\right)= & k_{e} \sum_{\mathbf{b}} \sum_{k}^{k \neq i \text { if } \mathbf{b}=\mathbf{0}} \frac{q_{k}}{\left|\mathbf{r}_{i k}+\mathbf{b} L\right|} f\left(\left|\mathbf{r}_{i k}+\mathbf{b} L\right|\right) \\
& +k_{e} \sum_{\mathbf{b}} \sum_{k}^{k \neq i \text { if } \mathbf{b}=\mathbf{0}} \frac{q_{k}}{\left|\mathbf{r}_{i k}+\mathbf{b} L\right|}\left(1-f\left(\left|\mathbf{r}_{i k}+\mathbf{b} L\right|\right)\right) .
\end{aligned}
$$

The function $f\left(\left|\mathbf{r}_{i k}+\mathbf{b} L\right|\right)$ is chosen such that the first series converges rapidly in the real space, while sum of the Fourier transforms of the terms in the second series converge rapidly in reciprocal space. That is, the first sum is simply performed in real space, while the terms in the second sum are evaluated in reciprocal space. After evaluating the Fourier transforms of the terms in the second sum, their total provides the inverse Fourier transform, which is then added to the first sum. Usually, $f\left(\left|\mathbf{r}_{i k}+\mathbf{b} L\right|\right)=\operatorname{erfc}\left(\beta\left|\mathbf{r}_{i k}+\mathbf{b} L\right|\right)$, where

$$
\operatorname{erfc}(x)=1-\frac{2}{\sqrt{\pi}} \int_{0}^{x} e^{-u^{2}} d u
$$

denotes the complementary error function, and $\beta$ is the Ewald splitting parameter. ${ }^{1}$ The final result reads

$$
\begin{aligned}
U\left(\mathbf{r}_{i}\right)= & k_{e} \sum_{\mathbf{b}} \sum_{k}^{k \neq i \text { if } \mathbf{b}=\mathbf{0}} q_{k} \frac{\operatorname{erfc}\left(\beta\left|\mathbf{r}_{i k}+\mathbf{b} L\right|\right)}{\left|\mathbf{r}_{i k}+\mathbf{b} L\right|} \\
& +k_{e} \frac{4 \pi}{L^{3}} \sum_{\mathbf{m} \neq \mathbf{0}} \sum_{k} \frac{q_{k}}{|\mathbf{m}|^{2}} \exp \left(-|\mathbf{m}|^{2} / 4 \beta^{2}\right) \exp \left(i \mathbf{m} \cdot \mathbf{r}_{i k}\right) \\
& -k_{e} \frac{2 \beta}{\sqrt{\pi}} q_{i},
\end{aligned}
$$

where $\mathbf{m}=2 \pi \mathbf{b} / L$, and the last term corrects for the self-interaction. The force on the

\footnotetext{
${ }^{1}$ In all the simulations in the present thesis, the size of the system is $70 d_{0} \times 70 d_{0} \times 70 d_{0}$. The value of the Ewald splitting parameter is chosen to be 0.125 , which is sufficient for the real part of the electrostatic potential to drop to a negligible value within the standard minimum image convention.
} 
particle $i$ is then obtained using Eq. (3.15), which provides

$$
\begin{aligned}
\mathbf{F}_{i}^{\text {long }}= & -k_{e} q_{i} \sum_{\mathbf{b}} \sum_{k}^{k \neq i \text { if } \mathbf{b}=\mathbf{0}} q_{k} \frac{\mathbf{r}_{i k, \mathbf{b}}}{\left|\mathbf{r}_{i k, \mathbf{b}}\right|^{3}}\left[\operatorname{erfc}\left(\beta\left|\mathbf{r}_{i k, \mathbf{b}}\right|\right)+\frac{2 \beta}{\sqrt{\pi}\left|\mathbf{r}_{i k, \mathbf{b}}\right|} \exp \left(-\beta^{2}\left|\mathbf{r}_{i k, \mathbf{b}}\right|^{2}\right)\right] \\
& -k_{e} q_{i} \frac{4 \pi}{L^{3}} \sum_{\mathbf{m}}^{\mathbf{m} \neq \mathbf{0}} \sum_{k}^{k \neq i} q_{k} \frac{\mathbf{m}}{|\mathbf{m}|^{2}} \exp \left[-\frac{|\mathbf{m}|^{2}}{4 \beta^{2}}\right] \sin \left(\mathbf{m} \cdot \mathbf{r}_{i k}\right)
\end{aligned}
$$

where $\mathbf{r}_{i k, \mathbf{b}} \equiv \mathbf{r}_{i k}+\mathbf{b} L=\left(\mathbf{r}_{k}-\mathbf{r}_{i}\right)+\mathbf{b} L$. Notice that the gradient of the self-correction term is zero, and the so called surface dipole corrections are neglected. ${ }^{1}$

\subsection{Long-range forces: Induced polarization}

In chapter 7 , the case of electrically polarized granular gas is addressed, up to the dipole level. In addition to the charge-charge interactions (CC), the charge-dipole interactions (CD), the dipole-charge interactions (DC), and the dipole-dipole interactions (DD) are added. The grain material is assumed to have isotropic polarizibility $\alpha_{\mu}$. If the electric field at the location of grain $i$ due to surrounding charges, and dipoles, is $\mathbf{e}_{i}\left(\mathbf{r}_{i}\right)$, then the induced dipole moment $\boldsymbol{\mu}_{i}\left(\mathbf{r}_{i}\right)$ is written as

$$
\boldsymbol{\mu}_{i}\left(\mathbf{r}_{i}\right)=\alpha_{\mu} \mathbf{e}_{i}\left(\mathbf{r}_{i}\right)
$$

The electric field $\mathbf{e}_{i}\left(\mathbf{r}_{i}\right)$, for a given charge and dipole configuration, is computed as

$$
\mathbf{e}_{i}=-\sum_{k} \sum_{\mathbf{b}}^{k \neq i \text { if } \mathbf{b}=\mathbf{0}} k_{e} \frac{q_{k}}{\left|\mathbf{r}_{i k}+\mathbf{b} L\right|^{3}}\left(\mathbf{r}_{i k}+\mathbf{b} L\right)+\sum_{k, k \neq i}^{r_{i k} \leq r_{c}} k_{e}\left[\frac{\boldsymbol{\mu}_{k}}{r_{i k}^{3}}-\frac{3 \mathbf{r}_{i k}\left(\mathbf{r}_{i k} \cdot \boldsymbol{\mu}_{k}\right)}{r_{i k}^{5}}\right] .
$$

Eqations (3.21) and (3.22) are coupled and must be solved simultaneously. In the simulations, the Jacobi iteration method is used to solve Eqs. (3.21) and (3.22). In Eq. (3.22), the Ewald summation is utilized for the computation of the $\mathrm{CC}$ interations (first term on RHS), while the CD interactions (second term on RHS) are computed up to a cutoff radius $r_{c}$. In the simulation $r_{c}=L / 2$, i.e., the standard minimum image convention. Once Eqs. (3.21) and (3.22) are solved in a coupled manner, the polarization force on a grain $i$

\footnotetext{
${ }^{1}$ See Appendix 3 for a simple script to move from Eq. (3.19) to Eq. (3.20).
} 
(a) Charge-charge

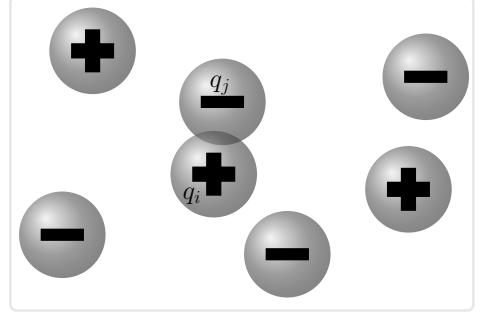

(c) Dipole-charge (b) Charge-dipole

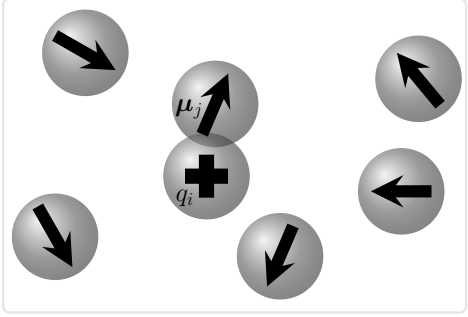

(d) Dipole-dipole

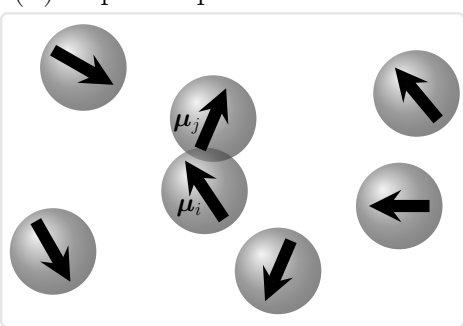

Figure 3.2: A schematic representation of the treatment of long-range charge-charge interactions (CC), charge-dipole interactions (CD), dipole-charge interactions (DC), and dipole-dipole interactions (DD). While CC interactions are dealt with using the Ewald summation, the minimum image convention is used for the other three cases.

due to $\mathrm{CD}, \mathrm{DC}$, and $\mathrm{DD}$ interactions is computed as

$$
\begin{aligned}
\mathbf{F}_{i}^{\mathrm{pol}}= & \sum_{k, k \neq i}^{r_{i k} \leq r_{c}} k_{e} q_{i}\left[\frac{\boldsymbol{\mu}_{k}}{r_{i k}^{3}}-\frac{3 \mathbf{r}_{i k}\left(\mathbf{r}_{i k} \cdot \boldsymbol{\mu}_{k}\right)}{r_{i k}^{5}}\right] \\
& -\sum_{k, k \neq i}^{r_{i k} \leq r_{c}} k_{e} q_{k}\left[\frac{\boldsymbol{\mu}_{i}}{r_{i k}^{3}}-\frac{3 \mathbf{r}_{i k}\left(\mathbf{r}_{i k} \cdot \boldsymbol{\mu}_{i}\right)}{r_{i k}^{5}}\right] \\
& -\sum_{k, k \neq i}^{r_{i k} \leq r_{c}} k_{e} \boldsymbol{\mu}_{i} \cdot\left[3 \frac{\mathbf{r}_{i k} \otimes \boldsymbol{\mu}_{k}+\boldsymbol{\mu}_{k} \otimes \mathbf{r}_{i k}}{r_{i k}^{5}}+3 \frac{\mathbf{r}_{i k} \otimes \boldsymbol{\mu}_{k}}{r_{i k}^{5}} \overline{\mathbf{I}}-15 \frac{\mathbf{r}_{i k} \otimes \boldsymbol{\mu}_{k}}{r_{i k}^{7}} \mathbf{r}_{i k} \otimes \mathbf{r}_{i k}\right],
\end{aligned}
$$

where the operator $\otimes$ is the tensor product, and $\overline{\mathbf{I}}$ is the identity matrix. The first term in Eq. (3.23) is the force on the charge $i$ due to the surrounding dipoles $k$, the second term is the force on the dipole $i$ due to the surrounding charges $k$, while the last term is the force on the dipole $i$ due to the surrounding dipoles $k$. Finally, the total long-range force on a grain is the sum of the Eqs. (3.20) and (3.23), i.e.

$$
\mathbf{F}_{i}^{\text {long }}+\mathbf{F}_{i}^{\mathrm{pol}}
$$




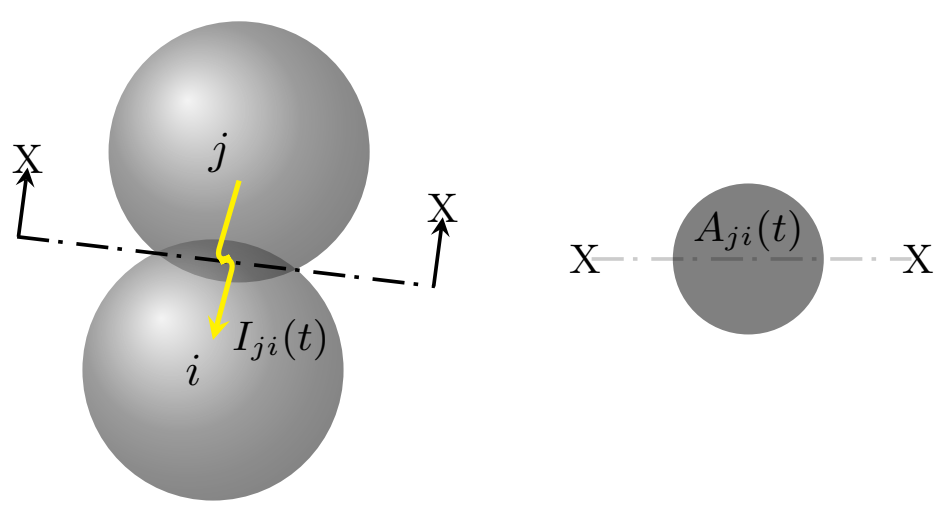

Figure 3.3: The charge exchange current $I_{j i}(t)$ during the course of collision between two grains $i$ and $j$. The time dependent contact area $A_{j i}(t)$ during the course of collision is shown on the right, indicated by section XX. The instantaneous area during collision can be related to the mutual particle compression $\xi_{i j}$ as: $A_{j i}(t)=\pi \xi_{i j}(t)\left[d_{0}-\xi_{i j}(t)\right]$. See also Eq. (3.5) and Fig. 3.1 for $\xi_{i j}$.

\subsection{Charge currents}

In this work, it is also considered that the electric charge on a grain evolves with time due to collisional charge exchange between the particles. If $I_{j i}(t)$ are the instantaneous currents from neighbor particles $j$ colliding with particle $i$, then we write the equation of motion for the charge $q_{i}$ on a grain $i$ as

$$
\frac{d q_{i}}{d t}=I_{i}=\sum_{j} I_{j i}(t) \Theta\left(d_{0}-r_{i j}\right) .
$$

In this thesis, certain treatments of the charge exchange currents have been implemented, which are summarized in Table 3.1. The corresponding descriptions of the models are presented in the thesis, whenever they are used for the first time.

\subsection{Rescaling of the equations: Non-dimensional num- bers}

In the following, the system of equations of motion are non-dimensionalized and important non-dimensional parameters are deduced. Introducing the following non-dimensional 
variables

$$
\begin{aligned}
& \tilde{m}_{i}=\frac{m_{i}}{m_{\mathrm{ref}}}, \tilde{\mathbf{v}}_{i}=\frac{\mathbf{v}_{i}}{v_{\mathrm{ref}}}, \tilde{t}=\frac{t}{t_{\mathrm{ref}}}, \tilde{\mathbf{r}}_{i j}=\frac{\mathbf{r}_{i j}}{\ell_{\mathrm{ref}}}, \\
& \tilde{\xi}_{i j}=\frac{\xi_{i j}}{\ell_{\mathrm{ref}}}, \tilde{d}_{0}=\frac{d_{0}}{\ell_{\mathrm{ref}}}, \tilde{\mathbf{n}}_{i j}=\frac{\tilde{\mathbf{r}}_{i j}}{\left|\tilde{\mathbf{r}}_{i j}\right|}, \tilde{L}=\frac{L}{\ell_{\mathrm{ref}}}, \\
& \tilde{q}_{i}=\frac{q_{i}}{q_{\mathrm{ref}}}, \tilde{\boldsymbol{\mu}}_{i}=\frac{\boldsymbol{\mu}_{i}}{\mu_{\mathrm{ref}}}, \tilde{\mathbf{e}}_{i}=\frac{\mathbf{e}_{i}}{e_{\mathrm{ref}}}, \tilde{I}_{j i}=\frac{I_{j i}}{I_{\mathrm{ref}}}, \\
& \tilde{r}_{c}=\frac{r_{c}}{\ell_{\mathrm{ref}}}
\end{aligned}
$$

the rescaled form of the equation of motion reads as

$$
\begin{aligned}
& \tilde{m}_{i} \frac{d \tilde{\mathbf{v}}_{i}}{d \tilde{t}}= \\
& -\left[\frac{t_{\text {ref }} E \ell_{\text {ref }}^{3 / 2}}{m_{\text {ref }} v_{\text {ref }}}\right] \sum_{j} \tilde{\xi}_{i j}^{\frac{3}{2}} \tilde{\mathbf{n}}_{i j} \Theta\left(\tilde{d}_{0}-\tilde{r}_{i j}\right) \\
& -\left[\frac{t_{\text {ref }} D \ell_{\text {ref }}^{1 / 2} v_{\text {ref }}}{m_{\text {ref }} v_{\text {ref }}}\right] \sum_{j} \tilde{\xi}_{i j}^{\frac{1}{2}} \dot{\tilde{\xi}}_{i j} \tilde{\mathbf{n}}_{i j} \Theta\left(\tilde{d}_{0}-\tilde{r}_{i j}\right) \\
& -\left[\frac{t_{\mathrm{ref}} k_{e} q_{\mathrm{ref}}^{2}}{\ell_{\mathrm{ref}}^{2} m_{\mathrm{ref}} v_{\mathrm{ref}}}\right] \sum_{k} \sum_{\mathbf{b}}^{k \neq i \text { if } \mathbf{b}=\mathbf{0}} \tilde{q}_{i} \frac{\tilde{q}_{k}}{\left|\tilde{\mathbf{r}}_{i k}+\mathbf{b} \tilde{L}\right|^{3}}\left(\tilde{\mathbf{r}}_{i k}+\mathbf{b} \tilde{L}\right) \\
& +\left[\frac{t_{\mathrm{ref}} k_{e} q_{\mathrm{ref}} \mu_{\mathrm{ref}}}{\ell_{\mathrm{ref}}^{3} m_{\mathrm{ref}} v_{\mathrm{ref}}}\right] \sum_{k, k \neq i}^{\tilde{r}_{i k} \leq \tilde{r}_{c}} \tilde{q}_{i}\left[\frac{\tilde{\boldsymbol{\mu}}_{k}}{\tilde{r}_{i k}^{3}}-\frac{3 \tilde{\mathbf{r}}_{i k}\left(\tilde{\mathbf{r}}_{i k} \cdot \tilde{\boldsymbol{\mu}}_{k}\right)}{\tilde{r}_{i k}^{5}}\right] \\
& -\left[\frac{t_{\mathrm{ref}} k_{e} q_{\mathrm{ref}} \mu_{\mathrm{ref}}}{\ell_{\mathrm{ref}}^{3} m_{\mathrm{ref}} v_{\mathrm{ref}}}\right] \sum_{k, k \neq i}^{\tilde{r}_{i k} \leq \tilde{r}_{c}} \tilde{q}_{k}\left[\frac{\tilde{\boldsymbol{\mu}}_{i}}{\tilde{r}_{i k}^{3}}-\frac{3 \tilde{\mathbf{r}}_{i k}\left(\tilde{\mathbf{r}}_{i k} \cdot \tilde{\boldsymbol{\mu}}_{i}\right)}{\tilde{r}_{i k}^{5}}\right] \\
& -\left[\frac{t_{\mathrm{ref}} k_{e} \mu_{\mathrm{ref}}^{2}}{\ell_{\mathrm{ref}}^{4} m_{\mathrm{ref}} v_{\mathrm{ref}}}\right] \sum_{k, k \neq i}^{\tilde{r}_{i k} \leq \tilde{r}_{c}} \tilde{\boldsymbol{\mu}}_{i} \cdot\left[3 \frac{\tilde{\mathbf{r}}_{i k} \otimes \tilde{\boldsymbol{\mu}}_{k}+\tilde{\boldsymbol{\mu}}_{k} \otimes \tilde{\mathbf{r}}_{i k}}{\tilde{r}_{i k}^{5}}+3 \frac{\tilde{\mathbf{r}}_{i k} \otimes \tilde{\boldsymbol{\mu}}_{k}}{\tilde{r}_{i k}^{5}} \overline{\mathbf{I}}-15 \frac{\tilde{\mathbf{r}}_{i k} \otimes \tilde{\boldsymbol{\mu}}_{k}}{\tilde{r}_{i k}^{7}} \tilde{\mathbf{r}}_{i k} \otimes \tilde{\mathbf{r}}_{i k}\right],
\end{aligned}
$$

the polarization equation reads as

$$
\tilde{\boldsymbol{\mu}}_{i}=\left[\frac{\alpha_{\mu} e_{\mathrm{ref}}}{\mu_{\mathrm{ref}}}\right] \tilde{\mathbf{e}}_{i}
$$


the electric field equation reads as

$$
\begin{aligned}
\tilde{\mathbf{e}}_{i}= & -\left[\frac{k_{e} q_{\mathrm{ref}}}{\ell_{\mathrm{ref}}^{2} e_{\mathrm{ref}}}\right] \sum_{k}^{k \neq i \text { if } \mathbf{b}=\mathbf{0}} \sum_{\mathbf{b}} \frac{\tilde{q}_{k}}{\left|\tilde{\mathbf{r}}_{i k}+\mathbf{b} \tilde{L}\right|^{3}}\left(\tilde{\mathbf{r}}_{i k}+\mathbf{b} \tilde{L}\right) \\
& +\left[\frac{k_{e} \mu_{\mathrm{ref}}}{\ell_{\mathrm{ref}}^{3} e_{\mathrm{ref}}}\right] \sum_{k, k \neq i}^{\tilde{r}_{i k} \leq \tilde{r}_{c}}\left[\frac{\tilde{\boldsymbol{\mu}}_{k}}{\tilde{r}_{i k}^{3}}-\frac{3 \tilde{\mathbf{r}}_{i k}\left(\tilde{\mathbf{r}}_{i k} \cdot \tilde{\boldsymbol{\mu}}_{k}\right)}{\tilde{r}_{i k}^{5}}\right],
\end{aligned}
$$

and, the equation of motion for the charge reads as

$$
\frac{d \tilde{q}_{i}}{d \tilde{t}}=\left[\frac{t_{\mathrm{ref}} I_{\mathrm{ref}}}{q_{\mathrm{ref}}}\right] \sum_{j} \tilde{I}_{j i}(t) \Theta\left(\tilde{d}_{0}-\tilde{r}_{i j}\right) .
$$

Setting $\mu_{\text {ref }}=q_{\text {ref }} \ell_{\text {ref }}, e_{\text {ref }}=k_{e} q_{\text {ref }} / \ell_{\text {ref }}^{2}$, the equation of motion is further simplified as

$$
\begin{aligned}
\tilde{m}_{i} \frac{d \tilde{\mathbf{v}}_{i}}{d \tilde{t}}= & -\mathcal{E} \sum_{j} \tilde{\xi}_{i j}^{\frac{3}{2}} \tilde{\mathbf{n}}_{i j} \Theta\left(\tilde{d}_{0}-\tilde{r}_{i j}\right)-\mathcal{D} \sum_{j} \tilde{\xi}_{i j}^{\frac{1}{2}} \dot{\tilde{\xi}}_{i j} \tilde{\mathbf{n}}_{i j} \Theta\left(\tilde{d}_{0}-\tilde{r}_{i j}\right) \\
& -\mathcal{K}\left[\tilde{\mathbf{F}}_{i}^{\text {long }}+\tilde{\mathbf{F}}_{i}^{\text {pol }}\right],
\end{aligned}
$$

the polarization equation reads as

$$
\tilde{\boldsymbol{\mu}}_{i}=\mathcal{A} \tilde{\mathbf{e}}_{i},
$$

the electric field equation reads as

$$
\tilde{\mathbf{e}}_{i}=-\sum_{k} \sum_{\mathbf{b}}^{k \neq i \text { if } \mathbf{b}=\mathbf{0}} \frac{\tilde{q}_{k}}{\left|\tilde{\mathbf{r}}_{i k}+\mathbf{b} \tilde{L}\right|^{3}}\left(\tilde{\mathbf{r}}_{i k}+\mathbf{b} \tilde{L}\right)+\sum_{k, k \neq i}^{\tilde{r}_{i k} \leq \tilde{r}_{c}}\left[\frac{\tilde{\boldsymbol{\mu}}_{k}}{\tilde{r}_{i k}^{3}}-\frac{3 \tilde{\mathbf{r}}_{i k}\left(\tilde{\mathbf{r}}_{i k} \cdot \tilde{\boldsymbol{\mu}}_{k}\right)}{\tilde{r}_{i k}^{5}}\right],
$$

and, the equation of motion for the charge reads

$$
\frac{d \tilde{q}_{i}}{d \tilde{t}}=\mathcal{Q} \sum_{j} \tilde{I}_{j i}(t) \Theta\left(\tilde{d}_{0}-\tilde{r}_{i j}\right)
$$


The non-dimensional groups in the above equations are the important problem parameters, which are denoted in the thesis as

$$
\begin{gathered}
\mathcal{E}=\left[\frac{t_{\mathrm{ref}} E \ell_{\mathrm{ref}}^{3 / 2}}{m_{\mathrm{ref}} v_{\mathrm{ref}}}\right], \mathcal{D}=\left[\frac{t_{\mathrm{ref}} D \ell_{\mathrm{ref}}^{1 / 2} v_{\mathrm{ref}}}{m_{\mathrm{ref}} v_{\mathrm{ref}}}\right], \mathcal{K}=\left[\frac{t_{\mathrm{ref}} k_{e} q_{\mathrm{ref}}^{2}}{\ell_{\mathrm{ref}}^{2} m_{\mathrm{ref}} v_{\mathrm{ref}}}\right], \\
\mathcal{A}=\left[\frac{\alpha_{\mu} k_{e}}{\ell_{\mathrm{ref}}^{3}}\right], \mathcal{Q}=\left[\frac{t_{\mathrm{ref}} I_{\mathrm{ref}}}{q_{\mathrm{ref}}}\right] .
\end{gathered}
$$

In the present work, the above non-dimensional forms of Eqs. (3.32)-(3.35) are numerically solved, instead of their dimensional counterparts. In the past, the effect of variations of $\mathcal{E}$ and $\mathcal{D}$ in granular gases is extensively dealt with. In this work, the focus is primarily on the problem parameters $\mathcal{K}, \mathcal{Q}$ and $\mathcal{A}$. Some useful outcomes from the above rescaling of the equations can be deduced straightaway. Let us consider the following limiting cases:

(i) $\mathcal{K} \rightarrow 0, \mathcal{A} \rightarrow 0, \mathcal{Q} \rightarrow 0$ with finite $\mathcal{E}$ and $\mathcal{D}:$

This is the case of neutral granular gas. The Coulomb interactions, polarization as well as charge exchange are negligible. In the most simplistic case in this regime, the dynamics of the gas would approach that of an ideal elastic gas, if $\mathcal{D}=0$ with finite $\mathcal{E}$. The case of non-zero $\mathcal{D}$ is extensively studied in the past and exhibit numerous intriguing features such as pattern formation (Goldhirsch \& Zanetti, 1993; McNamara, 1993; Brito \& Ernst, 1998; Brey et al. , 1999; Brilliantov et al. , 2004), universal Gaussian velocity distributions in the long time limit (Nie et al. , 2002), multiscaling and self-similarity in collisions (Ben-Naim \& Krapivsky, 2000; Ben-Avraham et al. , 2003), non-equilibrium steady states and asymmetric velocity distributions under energy inputs (Grossman et al. , 1997), anomalous diffusion (Bodrova et al. , 2016; Brilliantov \& Pöschel, 2000a), ballistic aggregation of clusters as a whole (Paul \& Das, 2017), and dissimilarity between ensemble-averages and long-time averages of observables (non-ergodicity) (Bodrova et al. , 2015).

(ii) $\mathcal{A} \rightarrow 0, \mathcal{Q} \rightarrow 0$ with finite $\mathcal{E}, \mathcal{D}$, and $\mathcal{K}:$

This case corresponds to a charged granular gas of monopoles, with fixed charge on each of the grain, without any polarization. This case of constant charge on grains has been studied by Müller (2008). It is used for validation in chapter 4. 
(iii) $\mathcal{A} \rightarrow 0$ with finite $\mathcal{E}, \mathcal{D}, \mathcal{K}$ and $\mathcal{Q}$ :

This case corresponds to a granular gas where charge exchange between grains is permitted, without polarization, and is the focus of chapters 4 and 5 in the present thesis.

(iv) Finite $\mathcal{E}, \mathcal{D}, \mathcal{K}, \mathcal{Q}$ and $\mathcal{A}$ :

This case corresponds to a granular gas where charge exchange between grains, as well as induced polarization is incorporated, and is the focus of chapter 7.

\subsection{Numerical integration of the equations of motion}

Two numerical integration schemes for the coupled differential equations of motion are used in the thesis:

(i) Gear's integration scheme, and

(ii) Verlet integration scheme.

The latter is used only in case of validation simulations. Unless otherwise specified, the default integration scheme used in the thesis is the Gear's integration scheme. In the following, the two numerical time integration schemes are briefly discussed.

\subsubsection{Gear's integration scheme}

The Gear's integration scheme consists of two steps. In the first step, the particle positions $\mathbf{r}_{i}$, velocities $\dot{\mathbf{r}}_{i}$, accelerations $\ddot{\mathbf{r}}_{i}$, and the higher order time derivatives are predicted at time $t+\Delta t$ from their known values at time $t$, using Taylor expansions:

$$
\begin{array}{r}
\mathbf{r}_{i}^{\text {predicted }}(t+\Delta t)=\mathbf{r}_{i}(t)+\Delta t \dot{\mathbf{r}}_{i}(t)+\frac{1}{2} \Delta t^{2} \ddot{\mathbf{r}}_{i}(t)+\frac{1}{6} \Delta t^{3} \dddot{\mathbf{r}}_{i}(t)+\mathcal{O}\left(\Delta t^{4}\right), \\
\dot{\mathbf{r}}_{i}^{\text {predicted }}(t+\Delta t)=\dot{\mathbf{r}}_{i}(t)+\frac{1}{2} \Delta t^{2} \ddot{\mathbf{r}}_{i}(t)+\frac{1}{6} \Delta t^{3} \dddot{\mathbf{r}}_{i}(t)+\mathcal{O}\left(\Delta t^{4}\right), \\
\ddot{\mathbf{r}}_{i}^{\text {predicted }}(t+\Delta t)=\ddot{\mathbf{r}}_{i}(t)+\frac{1}{6} \Delta t^{3} \dddot{\mathbf{r}}_{i}(t)+\mathcal{O}\left(\Delta t^{4}\right), \\
\dddot{\mathbf{r}}_{i}^{\text {predicted }}(t+\Delta t)=\dddot{\mathbf{r}}_{i}(t)+\mathcal{O}\left(\Delta t^{4}\right) .
\end{array}
$$


From the predicted particle positions and velocities, the forces $\mathbf{F}_{i}$ on the particles are computed. From these forces, the newly predicted accelerations are

$$
\ddot{\mathbf{r}}_{i}^{\text {force }}=\frac{\mathbf{F}_{i}}{m_{i}}
$$

which in general are not accurate, and are corrected next in a corrector step. The corrected values of the particle positions and its time derivatives are finally obtained by addition of numbers which are proportional to the difference between the accelerations from the forces, $\ddot{\mathbf{r}}_{i}^{\text {force }}$, and the predicted accelerations $\ddot{\mathbf{r}}_{i}^{\text {predicted }}$ at $t+\Delta t$, i.e.

$$
\Delta \ddot{\mathbf{r}}_{i}=\ddot{\mathbf{r}}_{i}^{\text {force }}-\ddot{\mathbf{r}}_{i}^{\text {predicted }}
$$

The complete correction step reads as

$$
\begin{aligned}
\mathbf{r}_{i}^{\text {corrected }}(t+\Delta t) & =\mathbf{r}_{i}^{\text {predicted }}(t+\Delta t)+a_{0} \frac{1}{2} \Delta t^{2} \Delta \ddot{\mathbf{r}}_{i}, \\
\dot{\mathbf{r}}_{i}^{\text {corrected }}(t+\Delta t) & =\dot{\mathbf{r}}_{i}^{\text {predicted }}(t+\Delta t)+a_{1} \frac{1}{2} \Delta t^{2} \Delta \ddot{\mathbf{r}}_{i}, \\
\ddot{\mathbf{r}}_{i}^{\text {corrected }}(t+\Delta t) & =\ddot{\mathbf{r}}_{i}^{\text {predicted }}(t+\Delta t)+a_{2} \frac{1}{2} \Delta t^{2} \Delta \ddot{\mathbf{r}}_{i}, \\
\dddot{\mathbf{r}}_{i}^{\text {corrected }}(t+\Delta t) & =\dddot{\mathbf{r}}_{i}^{\text {predicted }}(t+\Delta t)+a_{3} \frac{1}{2} \Delta t^{2} \Delta \ddot{\mathbf{r}}_{i} .
\end{aligned}
$$

The above described integration scheme is of fourth order, i.e., the numerical error grows as $\mathcal{O}\left(\Delta t^{4}\right)$, and requires calculation of time derivative upto third order. In principle however, Gear's scheme of any order can be implemented. In the present thesis, a sixth order Gear's scheme is utilized. The coefficients $a_{k}$ depend on the order of the scheme and the nature of the differential equations. For a sixth order gear integration scheme, the coefficients are (Pöschel \& Schwager, 2005)

$$
a_{0}=\frac{3}{16}, a_{1}=\frac{251}{360 \Delta t}, a_{2}=\frac{1}{\Delta t^{2}}, a_{3}=\frac{11}{18 \Delta t^{3}}, a_{4}=\frac{1}{6 \Delta t^{4}}, a_{5}=\frac{1}{60 \Delta t^{5}} .
$$

\subsubsection{Velocity Verlet integration scheme}

In specific cases, the velocity Verlet time integration scheme is used because it is computationally time-saving. However, in the present study, the most time-consuming calculations 
are the computation of long-range forces. Therefore, the use of the lower order velocity Verlet is restricted to only validation case studies, while the higher order Gear's integration scheme is utilized in the final simulations. For completeness, the velocity Verlet scheme is described below.

In this scheme, the particle velocities at half time step $t+\Delta t / 2$ are first computed using the Taylor expansion, as

$$
\dot{\mathbf{r}}_{i}\left(t+\frac{\Delta t}{2}\right)=\dot{\mathbf{r}}_{i}(t)+\frac{\Delta t}{2} \ddot{\mathbf{r}}_{i}(t)
$$

The particle positions are then updated as

$$
\mathbf{r}_{i}(t+\Delta t)=\mathbf{r}_{i}(t)+\Delta t \dot{\mathbf{r}}_{i}\left(t+\frac{\Delta t}{2}\right)
$$

The forces, or accelerations $\ddot{\mathbf{r}}_{i}(t+\Delta t)$, are then estimated using the particle positions $\mathbf{r}_{i}(t+\Delta t)$ and velocities $\dot{\mathbf{r}}_{i}\left(t+\frac{\Delta t}{2}\right)$. And finally the velocities are corrected using the Taylor expansion

$$
\dot{\mathbf{r}}_{i}(t+\Delta t)=\dot{\mathbf{r}}_{i}\left(t+\frac{\Delta t}{2}\right)+\frac{\Delta t}{2} \ddot{\mathbf{r}}_{i}(t+\Delta t) .
$$

\subsection{Integration of the stochastic differential equation for charge}

The charge-currents $I_{j i}(t)$ to build a charge distribution in the gas are considered stochastic in nature. Therefore, the numerical integration of the stochastic differential Eq. (3.35) requires special treatment. For brevity, the " $\sim$ " symbols are dropped from Eq. (3.35) in the following discussion.

As a first approximation, the charge-exchange currents $I_{j i}(t)$ are assumed to be white Gaussian random variables. It shall be shown shortly that such stochastic treatment of $I_{j i}(t)$ is not physical, and needs to be modified. However, the integration scheme for Eq. (3.35) described below is in the context of white Gaussian $I_{j i}(t)$. The integration scheme then can be modified for different treatments of $I_{j i}(t)$. If a different treatment of $I_{j i}$ is used, it is mentioned explicitly in the thesis. 
The white Gaussian currents $I_{j i}(t)$ are characterized by the following:

(i) Their expectation value $\left\langle I_{j i}(t)\right\rangle=0$ at any time $t$,

(ii) the variance $\left\langle I_{j i}(t)^{2}\right\rangle=\lambda$ at any time $t$, and

(iii) two values of the current $I_{j i}\left(t_{1}\right)$ and $I_{j i}\left(t_{2}\right)$ are independent of each other for $t_{1} \neq t_{2}$.

The noise $I_{j i}$ is thus discontinuous everywhere by definition, and its instantaneous values are not well defined.

Numerically, a discrete sequence of random numbers, say $W_{i}$, is used to replicate the properties of $I_{j i}(t)$, with intermediate time elapse $\Delta t$ between two consecutive random numbers $W_{1}$ and $W_{2}$. The first condition of $I_{j i}(t)$ having zero mean is simply fulfilled by choosing $W_{i}$ with zero mean. The variance of the discrete sequence $W_{i}$, however, is to be adjusted with $\Delta t$ (Volpe \& Volpe, 2013) for an accurate numerical solution of the stochastic differential equation of type (3.35). To mimic the second property, a condition $\left\langle\left(W_{i} \Delta t\right)^{2}\right\rangle / \Delta t=\lambda$ is to be imposed, which adjusts the variance of $W_{i}$ to $\lambda / \Delta t$ (Volpe $\&$ Volpe, 2013). In practice, a sequence of random numbers, uniformly distributed between 0 and 1 is generated by the machine. A suitable algorithm (here the Box-Muller transform) is then applied to transform these numbers to Gaussian white random numbers with zero mean and unit variance. Finally, these machine-generated numbers, say $w_{i}$, are rescaled to obtain $W_{i}=w_{i} \sqrt{\frac{\lambda}{\Delta t}}$, which satisfies $\left\langle\left(W_{i} \Delta t\right)^{2}\right\rangle / \Delta t=\lambda$. A finite-difference discretization of Eq. (3.35) thus can be written as

$$
q_{i}(t+\Delta t)=q_{i}(t)+\Delta t \mathcal{Q} W_{i}=q_{i}(t)+\Gamma w_{i}
$$

where $w_{i}$ are now the machine generated Gaussian numbers with zero mean and unit variance, and

$$
\Gamma=\sqrt{\lambda \Delta t} \mathcal{Q}
$$

In addition to above, if the grains have a finite capacity to hold a maximum charge of magnitude $\mathcal{C}$, the Constraint $\left|q_{i}(t)+\Gamma w_{i}\right| \leq \mathcal{C}$ should also be imposed. Thus Eq. (3.45) 
is written as

$$
\begin{aligned}
& q_{i}(t+\Delta t)=q_{i}(t)+\Gamma w_{i}, \text { if }\left|q_{i}(t+\Delta t)\right| \leq \mathcal{C}, \\
& q_{i}(t+\Delta t)=0, \text { otherwise. }
\end{aligned}
$$

Notice again that the above equation is already non-denationalized, and the nondimensional charge holding capacity of a grain is defined as

$$
\mathcal{C}=\frac{C}{q_{\text {ref }}}
$$

where $C$ is the charge holding capacity in Coulombs.

\subsection{Non physicality of random walk charge-exchange}

At present, there is extensive ongoing experimentation to explore the mechanism of collisional charging of dielectric grains [Fig. 3.4]. As the mechanism itself remains elusive, the simulations of the collective dynamics of a gas made up of such grains are challenging. Additional complexities are added by induced polarization of grains and the non-equilibrium interstitial flow. The problem, even considering point-like charges, is not well addressed.

The stochastic nature of charging of grains is indicated in a recent study by Haeberle et al. (2018). Thus one might first test a Langevin-like, or random walk-like, model for the equation of motion for the charge on a particle. Results from a typical simulation of a granular gas, using Eq. (3.47) coupled with Eq. (3.32) are shown in Fig. 3.5 and Fig. 3.6-3.8.

In the long time limit, the neutral granular gases are characterized by a power-law decay of the granular temperature $T$. This result was first predicted by Haff (Haff, 1983), and is marked with black filled circles in Fig. 3.5. When random walk charge exchange currents are considered between the colliding particles, it is found that the granular temperature $T$ reaches a steady state at long times. This mechanism of charge-exchange essentially leads to energy injection into the system, which balances the dissipation. This is shown with blue circles and asterisks in Fig. 3.5 (a). The currents $I_{j i}$ are always active 


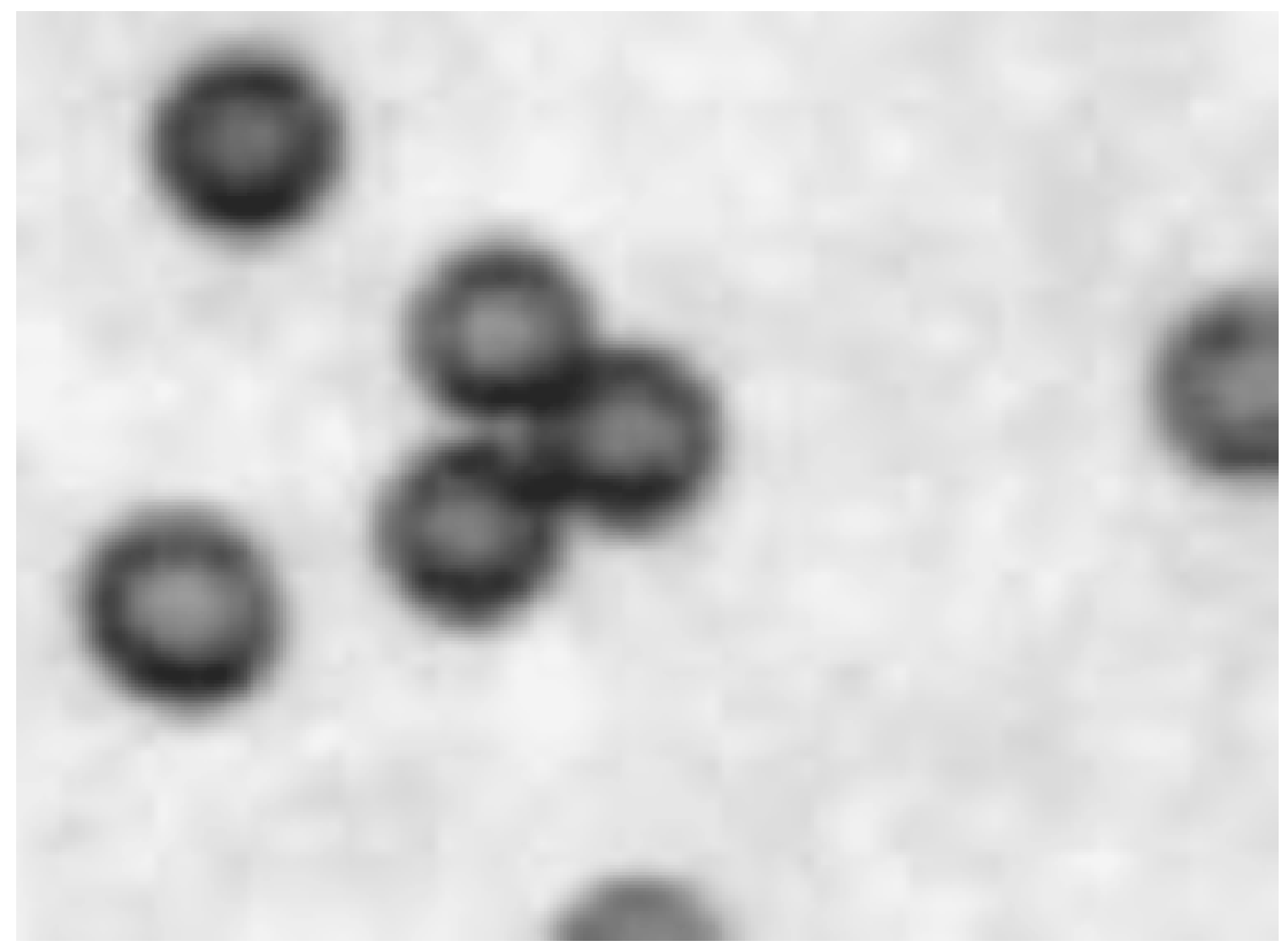

Figure 3.4: Charging and sticking of glass particles in a drop tower experiments (Image from private communication with Prof. Gerhard Wurm, Universität Duisburg Essen). URL: https://www.uni-due.de/imperia/md/images/physik/agw/sticking.gif. 


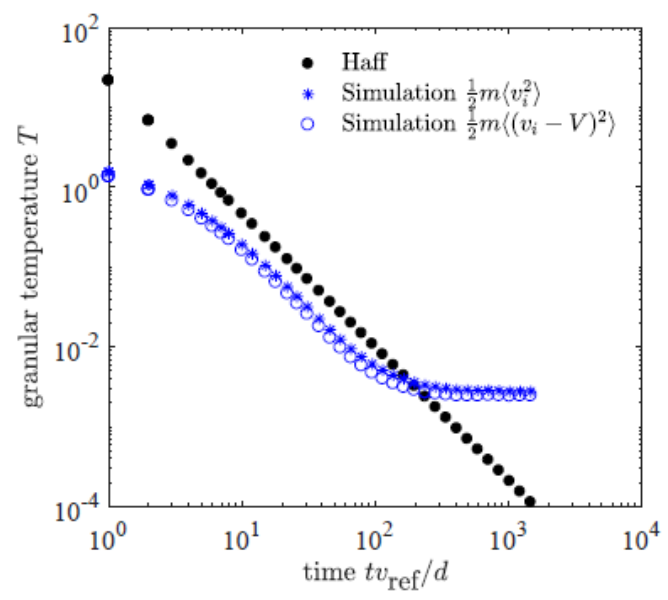

(a)

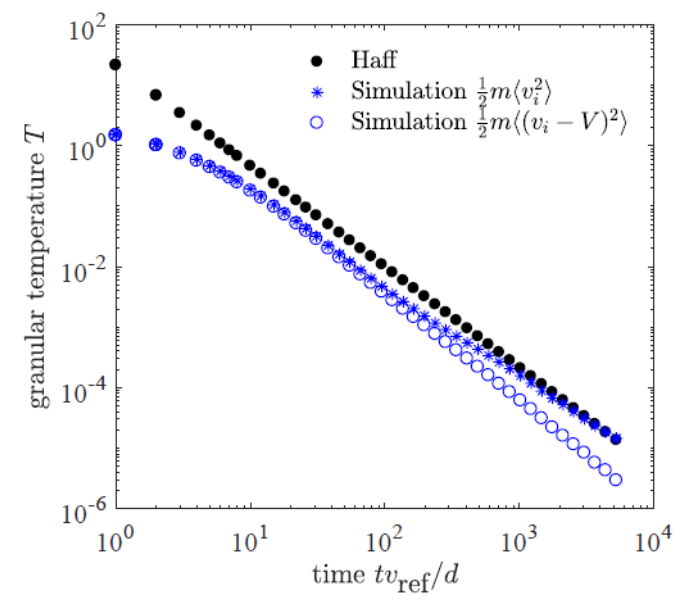

(b)

Figure 3.5: (a) The average kinetic energy and the granular temperature of a granular gas with random walk charge-exchange currents. This mechanism of charge-exchange leads to energy injection into the system, which balances the dissipation at long times. (b) The average kinetic energy and the granular temperature of a granular gas with modified charge-exchange currents. This mechanism of charge-exchange reproduces Haff's law at long times. Here $v_{i}$ is the grain speed and $V$ is the mean advective velocity in the vicinity of grain $i$.

during the collision events, and because the charges are coupled to EOM, they homogenize the system. The charge distribution attains a stationary state, however, the charge fluctuations persist. Coupled to the Coulomb forces, these fluctuations keep adding energy into the system. This continuous injection of energy is nonphysical for a real dissipative system.

In the above random-walk charge-exchange model, the role of initial conditions, $f(t=$ $0, q$ ), where $f$ is the charge distribution function, is also studied. The initial charge states can be set using a generalized distribution

$$
f(t=0, q)=\frac{z}{2 \Gamma(1 / z)} e^{-(|q|)^{z}}
$$

Different initial charge distribution $f(t=0 ; q)$ are chosen by varying $z$. Here the value of $z$ is varied from 8 (nearly uniform) to $z \rightarrow 0$ (delta distribution). It is found that in this case the long time dynamics is independent of the initial charge distribution. The distribution attains a steady state in each case. For each case of $z$, the gas remains spatially homogeneous, except for some probable local heterogeneities [Fig. 3.6-3.8]. 


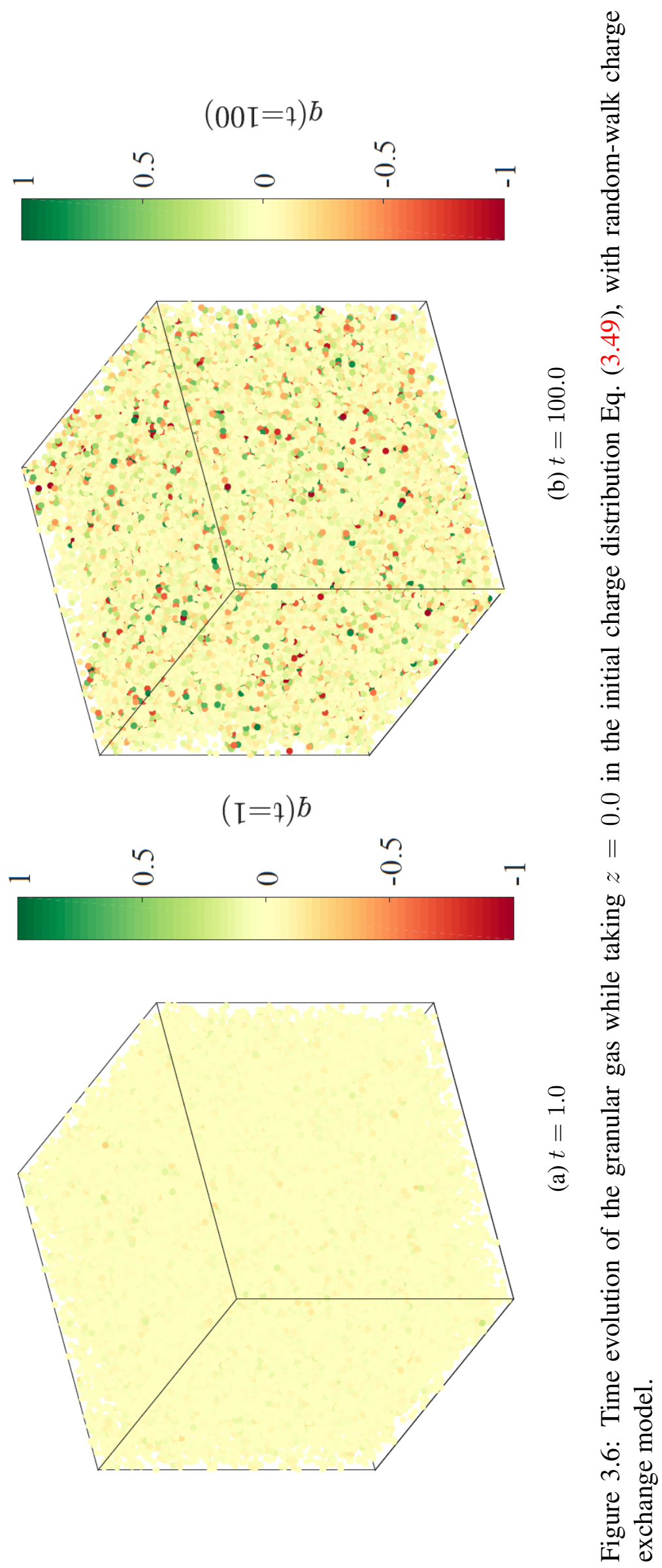




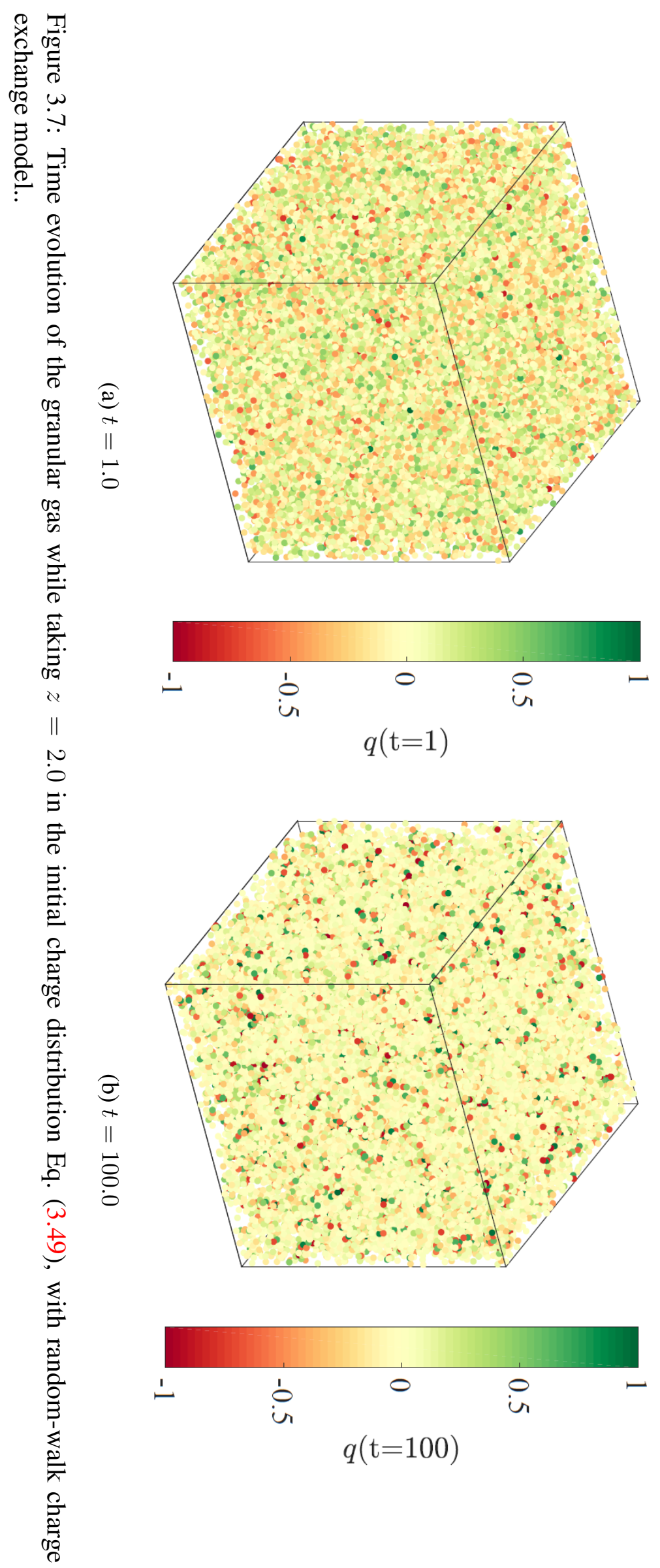



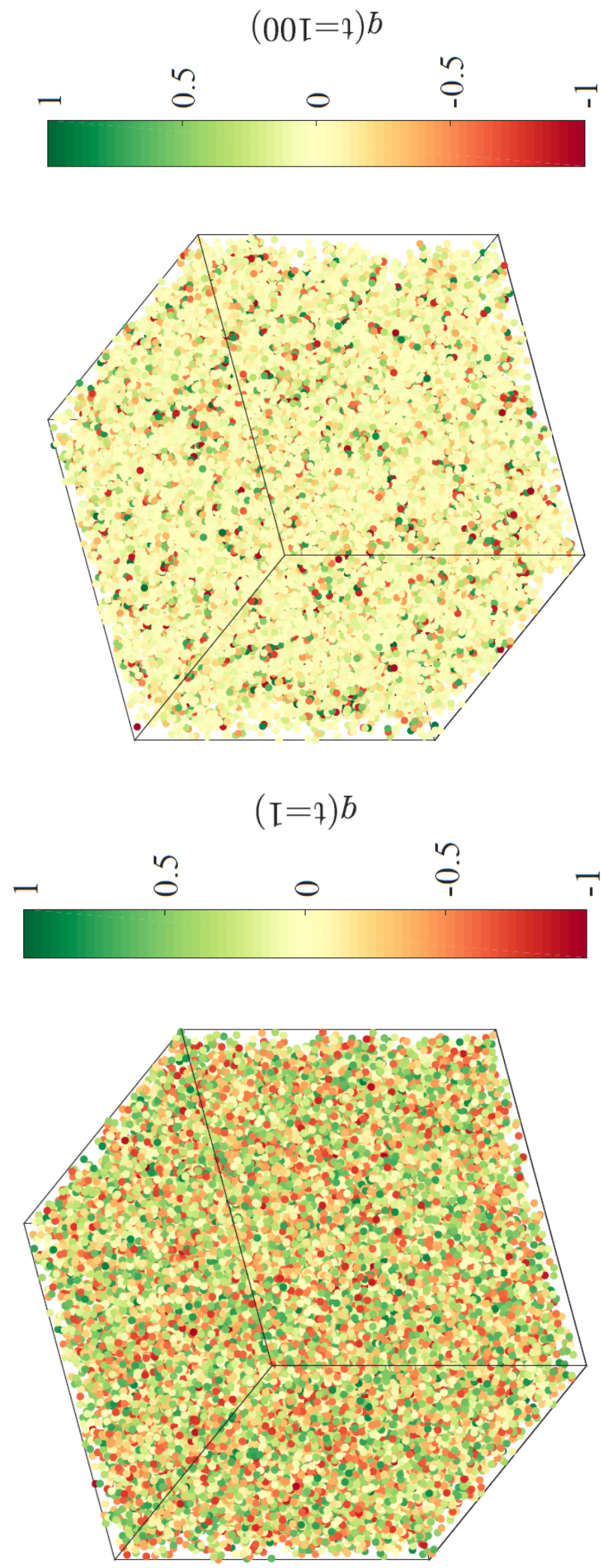

$\infty \stackrel{000}{\Xi}$. 突

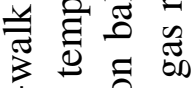

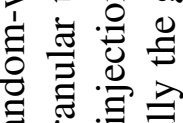

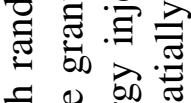

言造

字这

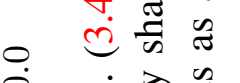

官 穴矛芯造

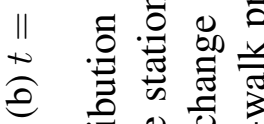

苟泀

.

ปี่

옹

离

‡

.

$\infty$ 휴

$\|$ 苞

on

స్ㅠ.

욜 疍

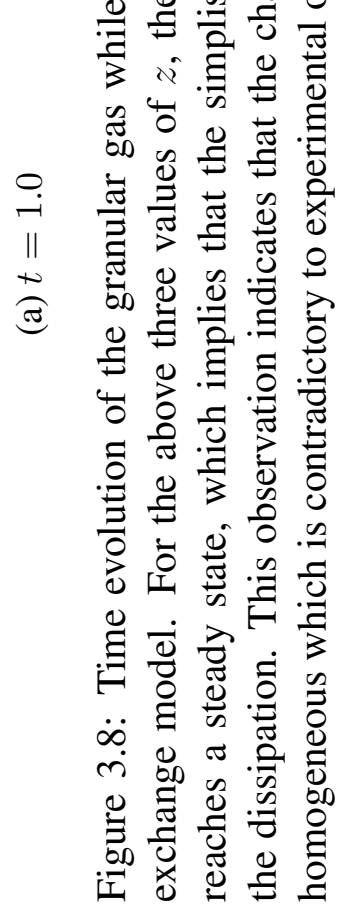




\subsection{Charge-exchange with multiplicative noise}

First, the charge exchange current $I_{j i}(t)$ from particle $j$ towards $i$ during the course of a collision can be rewritten (or dimensionally factorized) as

$$
I_{j i}(t)=A_{j i}(t) \frac{V_{j i}(t)}{\ell_{j i}(t) \rho_{\mathrm{I}}}
$$

where $A_{j i}(t), V_{j i}(t)$ and $\ell_{j i}(t)$ are the instantaneous collision contact area [Fig. 3.3], potential difference across the surfaces in contact, and a characteristic length along which the charge is transferred, while $\rho_{\mathrm{I}}$ is the particle's material resistivity. At the beginning of a collision, $A_{j i}=0$, and thus the charge transfer is zero. As the mutual particle compression $\xi_{i j}$ increases, $A_{j i}$ increases. As a modification to the random walk charge exchange model, the following approach is used. The contact area during a collision between grains is considered deterministic and is related to the mutual particle compression as

$$
A_{j i}(t)=\pi \xi_{i j}(t)\left[d_{0}-\xi_{i j}(t)\right]
$$

The experimental observation of the puzzling dynamics of charge sites on particle surface (Shinbrot et al. , 2018), and considering that the surface structure of dielectric particles in practice can be irregular, point to the fact that the potential difference and exchange length are hardly deterministic. This part, i.e. $\frac{V_{j i}(t)}{\ell_{j i}(t) \rho_{\mathrm{I}}}$, is now considered stochastic. A typical simulation result for granular temperature from a simulation with the above charge exchange approach is shown in Fig. 3.5 (b). The model reproduces the Haff's law and avoids any nonphysical energy injection into the system. The charge exchange models considered in this thesis are summarized in Table 3.1, and are elaborated wherever used in the thesis.

\subsection{Viscous friction: Preliminary}

In actual planetary dust aggregation processes, it is expected that the grain motion is influenced by its interactions with the surrounding gaseous medium. To mimic the simplest possible case, a viscous friction or drag force on the particles is also considered briefly in 


\section{Model}

$\frac{d q_{i}}{d t}=\sum_{j} I_{j i}(t) \Theta\left(d_{0}-r_{i j}\right)$,

$\left\langle I_{j i}(t)\right\rangle=0,\left\langle I_{j i}(t)^{2}\right\rangle=\lambda$

$$
\begin{gathered}
\frac{d q_{i}}{d t}=\sum_{j} I_{j i}(t) \Theta\left(d_{0}-r_{i j}\right) \propto \\
\sum_{j}(\text { rand sign })_{j} \times\left(v_{i j}^{2}\right)^{\eta}
\end{gathered}
$$

$$
\frac{d q_{i}}{d t}=\sum_{j} A_{j i}(t) \underbrace{\frac{V_{j i}(t)}{\ell_{j i}(t) \rho}}_{\Psi(t)} \Theta\left(d_{0}-r_{i j}\right)
$$

$$
\frac{d q_{i}}{d t}=0
$$

\section{Description}

Langevin like stochastic charge exchange with white Gaussian charge exchange currents $I_{j i}$. As shown in Fig. 3.5 (a), the model introduces a nonphysical energy injection into the system. This case is addressed in the present chapter.

Empirical charge exchange model based on experiments by Poppe et al. (2000b); Poppe \& Schräpler (2005)

where the researchers observed a dependence of charge exchange on the relative collision speeds. This case has been used in chapter 4 and 5 .

Charge-exchange currents proportional to the contact area during collision, with multiplicative noise term $\Psi(t)$. As shown in Fig. 3.5 (b), the model avoids nonphysical energy injection. This case is used in present chaper and chapter 7 .

No exchange with a given stationary $f(q)$. In this case, three choices of $f(q)$ are considered: (i) constant monopolar charge, (ii) stationary Gaussian, (iii) general charge distribution with a tuning parameter which alters the shape of the distribution from a uniform distribution towards a delta distribution.

This case is used for validation in chapter 4.

Table 3.1: Different models considered for the charge-exchange currents $I_{j i}$. Here $d_{0}$ is the grain diameter, $q_{i}$ is the charge on a grain $i$, and $r_{i j}$ is the distance between grains $i$ and $j$. 
chapter 7 , given by

$$
\mathbf{F}_{i}^{\text {fric }}=\frac{m_{i}}{\tau_{\text {fric }}}\left(\mathbf{v}_{\text {fluid }}-\mathbf{v}_{i}\right)
$$

where $\tau_{\text {fric }}$ is the friction time and can be related to different drag models. If $\tau_{\text {fric }} \rightarrow \infty$, it implies that the particle motion is negligibly influenced by the interstitial fluid velocity $\mathbf{v}_{\text {fluid }}$. On the other hand a small friction time would imply that the particle velocity $\mathbf{v}_{i}$ relaxes quickly to the fluid velocity $\mathbf{v}_{\text {fluid }}$. The Eq. (3.52) is rescaled as

$$
\tilde{\mathbf{F}}_{i}^{\text {fric }}=\left[\frac{t_{\text {ref }}}{\tau_{\text {fric }}}\right] \tilde{m}_{i}\left(\tilde{\mathbf{v}}_{\text {fluid }}-\tilde{\mathbf{v}}_{i}\right)
$$

The non-dimensional ratio of the two time scales is the Stokes number, denoted as

$$
\mathcal{S}=\left[\frac{t_{\text {ref }}}{\tau_{\text {fric }}}\right] .
$$

The effect of Stokes number is discussed briefly in chapter 7.

\subsection{Software and hardware used}

All the numerical simulations reported in this thesis are parallelized using CUDA (Compute Unified Device Architecture) parallel computing platform and are deployed on either Tesla-P100 or Tesla-K20Xm GPUs (Graphics Processing Units). The particle aggregation/cluster analysis is performed using an in-house MATLAB program. Apart from this the kinetic-theory [chapter 5], and the linear stability analysis of the hydrodynamic description [chapter 7], utilizes additional MATLAB and MATHEMATICA programs, which are provided in the appendices and online. 


\section{Chapter 4}

\section{Early-stage aggregation in}

\section{three-dimensional charged granular} $\operatorname{gas}^{1}$

Neutral grains made of the same dielectric material can attain considerable charges due to collisions and generate long-range interactions. We perform molecular dynamics simulations in three dimensions for a dilute, freely-cooling granular gas of viscoelastic particles that exchange charges during collisions. As compared to the case of clustering of viscoelastic particles solely due to dissipation, we find that the electrostatic interactions due to collisional charging alter the characteristic size, morphology and growth rate of the clusters. During the early stages of growth, the average cluster size grows with time as a power law, whose exponent is relatively larger in the charged gas than the neutral case. The growth of the average cluster size is found to be independent of the ratio of characteristic Coulomb to thermal energy, or equivalently, of the typical Bjerrum length. However, this ratio alters the crossover time of growth. Both simulations and mean-field calculations based on the Smoluchowski's equation suggest that suppression of particle diffusion, due to the electrostatic interactions, helps in the aggregation process.

\footnotetext{
${ }^{1}$ The content of this chapter has been published in: Singh, Chamkor, and Marco G. Mazza. "Early-stage aggregation in three-dimensional charged granular gas." Physical Review E 97.2 (2018): 022904.
} 


\subsection{Introduction}

Since classical antiquity lightning has been associated with the ashes produced during volcanic activity (Anderson et al. , 1965; Most, 2006). It has been long speculated that collisional charging may play a significant role in particle's aggregation (Gill, 1948; Wesson, 1973) in natural processes such as the formation of planetesimals during the early stages of the birth of a planet (Wesson, 1973; Poppe et al. , 2000b), charging in dust devils (Crozier, 1964), lightenings in thunderclouds (Simpson \& Scrase, 1937), and electric sparks in dunes (Kamra, 1972). At such length scales $\left(10^{2}-10^{4} \mathrm{~m}\right)$, a number of processes are observed such as charge separation, the buildup of significant potential differences and electric discharge (Franz et al. , 1990). A specific example is the electrostatic re-accretion in the protoplanetary disks where the charged fragmented ejecta from a larger body are reattracted towards the parent body due to its electrostatic field (Blum \& Wurm, 2008). On a more mundane scale, this phenomenon also affects the processes at length scales which are technologically relevant $e . g$. in vibrated granular beds (Kolehmainen et al. , 2016), in the transportation of coal (Nifuku et al. , 1989) and in electrostatic powder spraying (Bailey, 1998). The origin of the above intriguing processes inside a granular gas is due to the charging of particles during collisions. This phenomenon, however, is rather stochastic as indicated by experimental measurements (Lee et al. , 2015; Poppe et al. , 2000b; Hu et al. , 2012). On the other hand, the consequences of this local exchange on a collection of particles have been experimentally observed to be quite complex as it shows highly fluctuating characteristics (Nordsiek \& Lathrop, 2015). Very recently, these fluctuations also are shown numerically in dense granular systems (Yoshimatsu et al. , 2017). The collective consequences on particle aggregation and their growth due to this very local charge exchange in the dilute granular system, however, are not yet well understood. Relevant exceptions are the theoretical findings of Scheffler \& Wolf (2002); Müller (2008) for a monopolarly charged granular gas neglecting collisional charging, and the Smoluchowski's aggregation analysis by Dammer \& Wolf (2004) for the monopolarly charged suspensions undergoing Brownian motion.

A granular gas is an adequate theoretical setup to study such particle aggregation processes in the dilute limit. The clusters in a neutral granular gas typically exhibit a power law growth during its time evolution (Paul \& Das, 2017; Das et al. , 2016). It is, however, 
unclear if bipolar collisional charging of grains, which is ubiquitous in technological settings (Pingali et al. , 2009; Bailey, 1998; Nifuku et al. , 1989; Kolehmainen et al. , 2016; Mehrotra et al. , 2007), and in natural flows (Blum et al. , 2000; Blum \& Wurm, 2008; Poppe et al. , 2000a; Wolf et al. , 2009), enhances or suppresses the cluster growth.

The dissipation in neutral granular gases leads to the formation of clusters. The growth of these clusters in two and three dimensions has been investigated in the past (Miller \& Luding, 2004; Luding \& Herrmann, 1999; Brilliantov et al. , 2015; Brilliantov \& Spahn, 2006). In three-dimensions, it is found that the cluster growth can be described by a power law behavior with similar exponents as in percolation theory (Miller \& Luding, 2004). The same is shown for the growing clusters in two dimensions (Luding \& Herrmann, 1999). Furthermore, the coagulation equations for inelastic dust particles in a surrounding molecular gas, which is more relevant to natural settings, has also been studied from a mean-field point of view (Brilliantov \& Spahn, 2006). The growth of the clusters in granular gases has also been investigated for different nature of the interactions between the particles for example under short-range attractive potentials (Murphy \& Subramaniam, 2015) and square well potential (Takada et al. , 2016).

In this study, we show that the time-dependent average cluster size $S(t)$ in a charged granular gas, during the early stages of the growth, obeys the power law

$$
S(t) \sim t^{z}
$$

We elucidate that

(i) the early stage aggregation after the homogeneous cooling state (HCS) of the granular gas is relatively enhanced due to the collisional charging with $z$ changing from $\approx 6 / 5$ for the uncharged gas to $\approx 3 / 2$ for the charged gas,

(ii) the growth exponent $z$ is found to be independent of the ratio of the characteristic Coulomb to thermal energy $\mathcal{K}$ or equivalently the ratio $\mathcal{K}=\ell_{\mathrm{B}} / d_{0}$ of the Bjerrum length $\ell_{\mathrm{B}}=k_{\mathrm{e}} q_{\mathrm{ref}}^{2} / T_{\text {ref }}$ to the particle diameter $d_{0}$, where $q_{\mathrm{ref}}$ is the typical charge on the particles, $T_{\text {ref }}$ the thermal energy scale or the granular temperature, and $k_{e}=1 /\left(4 \pi \varepsilon_{0}\right)$ is the Coulomb constant with vacuum permittivity $\varepsilon_{0}=8.85418782 \times 10^{-12} \mathrm{~F} \mathrm{~m}^{-1}$. A change in $\ell_{B}$, however, influences the charac- 
teristic time of emergence of clustering.

(iii) We find that contrary to the case of neutral viscoelastic particles, the velocity distribution of the charged viscoelastic particles does not show a relaxation back towards the Maxwellian within the characteristic time of emergence of the inhomogeneous cooling state (ICS).

\subsection{Model}

We simulate $N_{\text {mon }} \sim 10^{4}-10^{5}$ identical, viscoelastic particles in a three-dimensional domain of volume $L^{3}=70 \times 70 \times 70 .^{1}$ The filling fraction of the system $\phi \equiv$ $N_{\text {mon }} \pi d_{0}^{3} /\left(6 L^{3}\right)=0.076$, and the ratio $\mathcal{E} / \mathcal{D} \approx 10$ are kept constant, ${ }^{2}$ while the effect of $\mathcal{K}$ on the particle aggregation is studied; the case $\mathcal{K}=0$ corresponds to the neutral viscoelastic granular gas. In addition, the particles attain charge during the pairwise collisions according to a charge exchange rule (of empirical basis augmented with stochasticity, Table 3.1), discussed below.

Collisional charging has far-reaching consequences. Large amounts of charges are generated in volcanic plumes (Anderson et al. , 1965); estimated figures are of the order of $10^{5}$ or $10^{6}$ elementary charges per cubic centimeter (Anderson et al. , 1965), and this effect might have played a role for the origin of life by synthesizing amino acids (Johnson et al. , 2008). Dust and sand storms also exhibit contact electrification and lightnings (Freier, 1960; Stow, 1969; Lacks \& Sankaran, 2011), and such phenomena might even exist on Mars (Melnik \& Parrot, 1998). Contact electrification can result in explosions if a flammable material is present (Glor, 1985; Nifuku et al. , 1989; Lacks \& Sankaran, 2011); pharmaceutical processes are often plagued by electrostatic charge buildup (Pu et al. , 2009; Pingali et al. , 2009) leading to high maintenance costs. Understandably, a vast amount of attention has been put to explore what mechanisms stimulate the charge buildup, separation, transport and its effect on the dynamics of granular flows

\footnotetext{
${ }^{1}$ Here $L$ is the non-dimensional linear size of the system. See rescaling in chpater 3.

${ }^{2}$ Taking silica particles as representative for the granular gas fixes Young's modulus $Y=73.1 \mathrm{GPa}$, Poisson's ratio $\nu_{\mathrm{P}}=0.2$, particle mass density $\rho_{\mathrm{m}}=2650 \mathrm{~kg} / \mathrm{m}^{3}$. We select a small value of the dissipation constant $\gamma=7.0 \times 10^{-6} \mathrm{~s}$. The dissipation constant $\gamma$ is introduced in Eq. (3.12) in chapter 3. The thermal energy scale or the initial granular temperature is $T(0)=10$. If we consider particle/monomer size $d_{0}=\mathcal{O}(\mathrm{mm})$, we find $\mathcal{E} \approx 278.6, \mathcal{D} \approx 27.7$. In all our calculations we fix $\mathcal{E}=278.6, \mathcal{D}=27.7$, and vary $\mathcal{K}$. See chapter 3 for definitions of $\mathcal{E}, \mathcal{D}$, and $\mathcal{K}$
} 

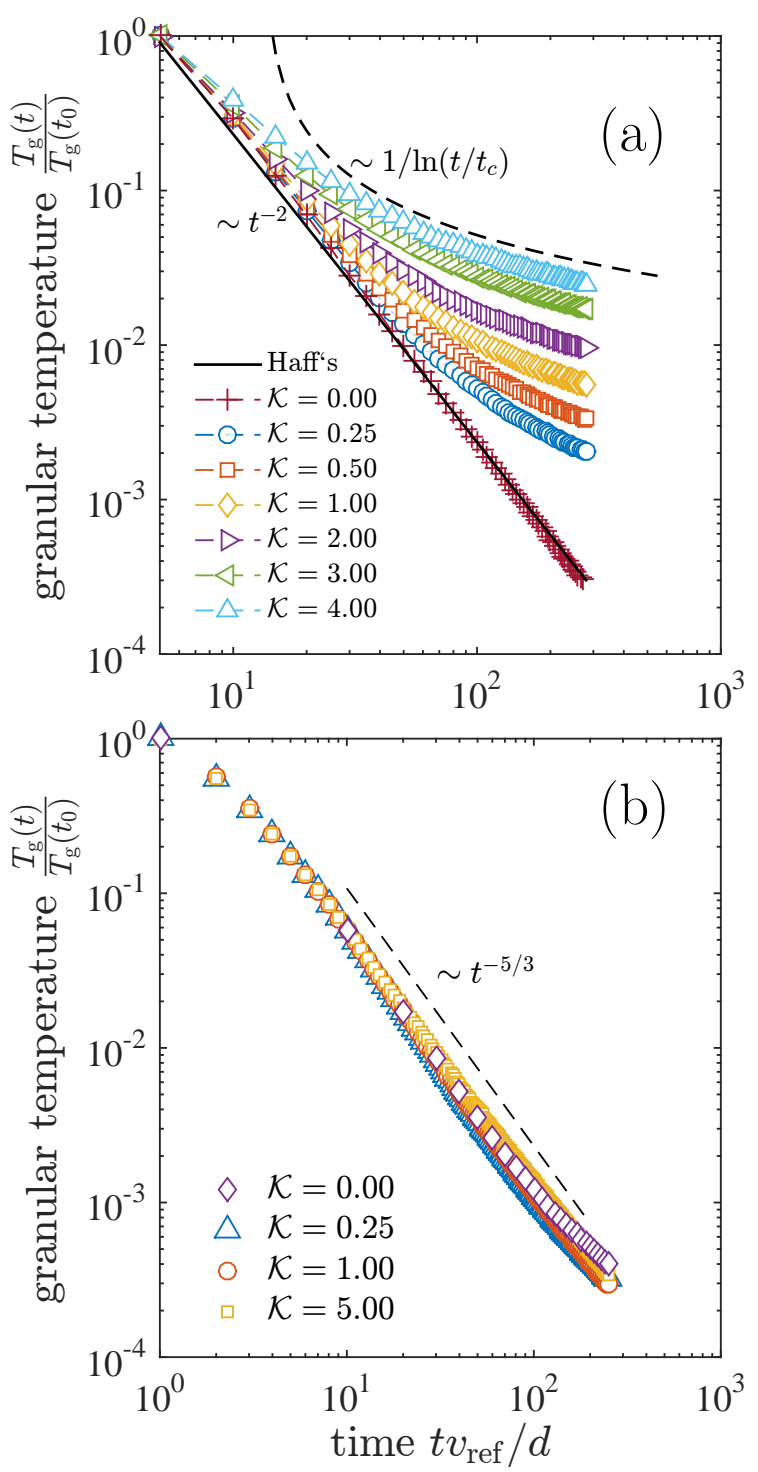

Figure 4.1: (a) The evolution of the granular temperature $T$ (or $T_{\mathrm{g}}$ ) for a purely repulsive dilute granular gas with monopolarly charged particles and constant coefficient of restitution $\epsilon$. We study the dependence on the ratio of Coulomb to thermal energy $\mathcal{K}$. The $\mathcal{K}=0$ curve corresponds to a neutral granular gas. At very short times, the granular gas follows Haff's (Haff, 1983) law $\left(T(t) \sim t^{-2}\right.$ ) in the homogeneous cooling state. The repulsive electrostatic interactions among the particles reduce the collision frequency and thus result in a slower decay of $T$ as time progresses $\left(T(t) \sim 1 / \ln \left(t / t_{c}\right)\right.$, also shown analytically by Scheffler $\&$ Wolf (2002)). As $\mathcal{K}$ increases, the deviation from Haff's law is more pronounced and occurs earlier in time. The solid line represents the theoretical prediction of Haff's law for a neutral granular gas with $\epsilon=$ const, and the dashed line is a theoretical prediction for monopolarly charged granular gases. (b) Same as (a) but for the early stage of evolution of the viscoelastic $(\epsilon \neq$ const.) granular gas with charge exchange. The dashed line represents the theoretical prediction of Haff's law for a neutral viscoelastic granular gas. Here the notations $T$ and $T_{\mathrm{g}}$ are used interchangeably, and $t_{0}$ is some time scale of relaxation of the initial transience in the gas. The time scale $t_{c}$ marks a transition from Haff's law to inverse logarithmic decay of $T$. 
(Gill, 1948; Anderson et al. , 1965; Kamra, 1972; Mehrotra et al. , 2007; Yoshimatsu et al. , 2017; Pähtz et al. , 2010). Additionally, the collective behavior is are unclear in spite of great experimental (Poppe et al. , 2000b; Nordsiek \& Lathrop, 2015; Lee et al. , 2015; Nordsiek \& Lathrop, 2015) and theoretical (Kolehmainen et al. , 2016; Müller, 2008; Scheffler \& Wolf, 2002; Yoshimatsu et al. , 2017; Takada et al. , 2017) efforts. Moreover, the theory of contact electrification, i.e., charging of similar or dissimilar surfaces due to mutual contact is not yet rigorously established. Two basic, experimental facts still defy a consistent explanation: $(i)$ insulators can transfer large amounts of charge, though they have no free charge carriers; (ii) upon contact/impact even the grains with identical material charge up (Pähtz et al. , 2010). However, there are certain observations which have been made repeatedly in the context of collisional charging. For instance, an extensive and systematic experimental study conducted by Poppe et al. (2000b); Poppe \& Schräpler (2005) has revealed that the number of elementary charges $\Delta Z_{i j}$ transferred during a collision of silica particles on polished quartz and silicon wafer surfaces, on average, are proportional to a power of the relative kinetic energy during the collision, i.e. $\Delta Z_{i j}=\left(K E_{\mathrm{kin}}\right)^{\eta}$, where $E_{\mathrm{kin}}$ is the relative kinetic energy during the collision, $K\left[\mathrm{~J}^{-1}\right]\left(K^{-1} \sim 10^{-12}-10^{-15} \mathrm{~J}\right)$ and $\eta=0.83$ are constants (Poppe et al. , 2000b). Similar observations have also been made by $\mathrm{Hu}$ et al. (2012) for single collisions of glass particles exhibiting dependence of charge transfer on impact energy. This, in one sense, is analogous with the impact velocity dependent coefficient of restitution. However, the widespread nature of data in the collisional charging experiments also suggests that the collisional charge exchange is influenced by myriad factors. Indeed, among possible influential parameters are the surface material and its roughness, contact pressure, surface cleaning, humidity, the orientation of the crystalline lattice, the temperature of the surfaces, and the size of the colliding objects (Poppe et al. , 2000b). Taking this into account, we introduce a collisional impact energy dependent model for the charge exchange augmented by a stochasticity in its parameters

$$
\Delta q_{i j}= \pm e \Delta Z_{i j}= \pm e\left[\Psi_{1} K \frac{1}{2} m^{\mathrm{eff}}\left(\mathbf{v}_{i j} \cdot \mathbf{n}_{i j}\right)^{2}\right]^{\eta+\Psi_{2}}
$$


or in non-dimensional terms ${ }^{1}$

$$
\Delta q_{i j}^{*}= \pm \mathcal{Q}\left[\Psi_{1} m^{\mathrm{eff} *}\left(\mathbf{v}_{i j}^{*} \cdot \mathbf{n}_{i j}^{*}\right)^{2}\right]^{\eta+\Psi_{2}},
$$

where $\mathcal{Q}=\frac{e\left(K m_{\mathrm{ref}} v_{\mathrm{ref}}^{2}\right)^{\eta}}{q_{\mathrm{ref}}}, m^{\text {eff }}=m_{i} m_{j} /\left(m_{i}+m_{j}\right)$ is the reduced mass of the colliding particles, $e=1.6021765 \times 10^{-19} \mathrm{C}$ is the absolute value of the electron charge, $\Delta Z_{i j}$ is the number of elementary charges exchanged, $m_{\text {ref }} v_{\text {ref }}^{2}=T_{\text {ref }}$ is the thermal energy scale. In our calculations, we fix $q_{\text {ref }}$ such that $\mathcal{Q}=\mathcal{O}(e K)$. The numbers $\Psi_{1}$ and $\Psi_{2}$ are equally distributed noise with $\left\langle\Psi_{1}\right\rangle=1.63,\left\langle\Psi_{2}\right\rangle=0$, in the intervals $\Psi_{1} \in[0.1,3.1623]$ and $\Psi_{2} \in[\eta-0.05 \eta, \eta+0.05 \eta]$. The mean and the interval of the noise $\Psi_{1}$ are chosen such that the charge exchange $\Delta q_{i j}$ fits the experimental power law $e \Delta Z_{i j}=e\left(K E_{\mathrm{kin}}\right)^{\eta}$ found by Poppe et al. (2000b).

The multiplicative noise $\Psi_{1}$ indicates that the charge exchange in the system has a dependence on the current state of the kinetic energies of collisions while the stochasticity in the exponent does not depend on the kinetic conditions, which is reflected through an additive noise $\Psi_{2}$. In other words, in a freely cooling granular gas, where the impact kinetic energies keep decreasing over time, the stochastic coupling coefficient $\Psi_{1}$ assures an upper limit to the charge on an individual particle and avoids any unphysical divergence of the amount of charge on it. It should be noted that here we make the simplifying assumption, in lack of any established theory of tribocharging, that the charge exchange is independent of the preexisting charge on the particles before the collisions. This simplification is partially based on the experiments of Poppe \& Schräpler (2005), where it was observed that even precharged particles acquire additional charge upon collision, indicating a negligible influence of electrical conductance during the short collision time. Therefore, neglecting the possibility of discharging of the particles due to the combined effect of potential created by the preexisting charge and the electrical conductivity of the particle's material is a reasonable simplification. The addition of the stochastic variable $\Psi_{2}$ in the exponent is based on the fact that its value is reported to be fluctuating ( 0.83 in Poppe et al. (2000b) while later 0.79 in Poppe \& Schräpler (2005)).

\footnotetext{
${ }^{1}$ See chapter 3 for rescaling.
} 

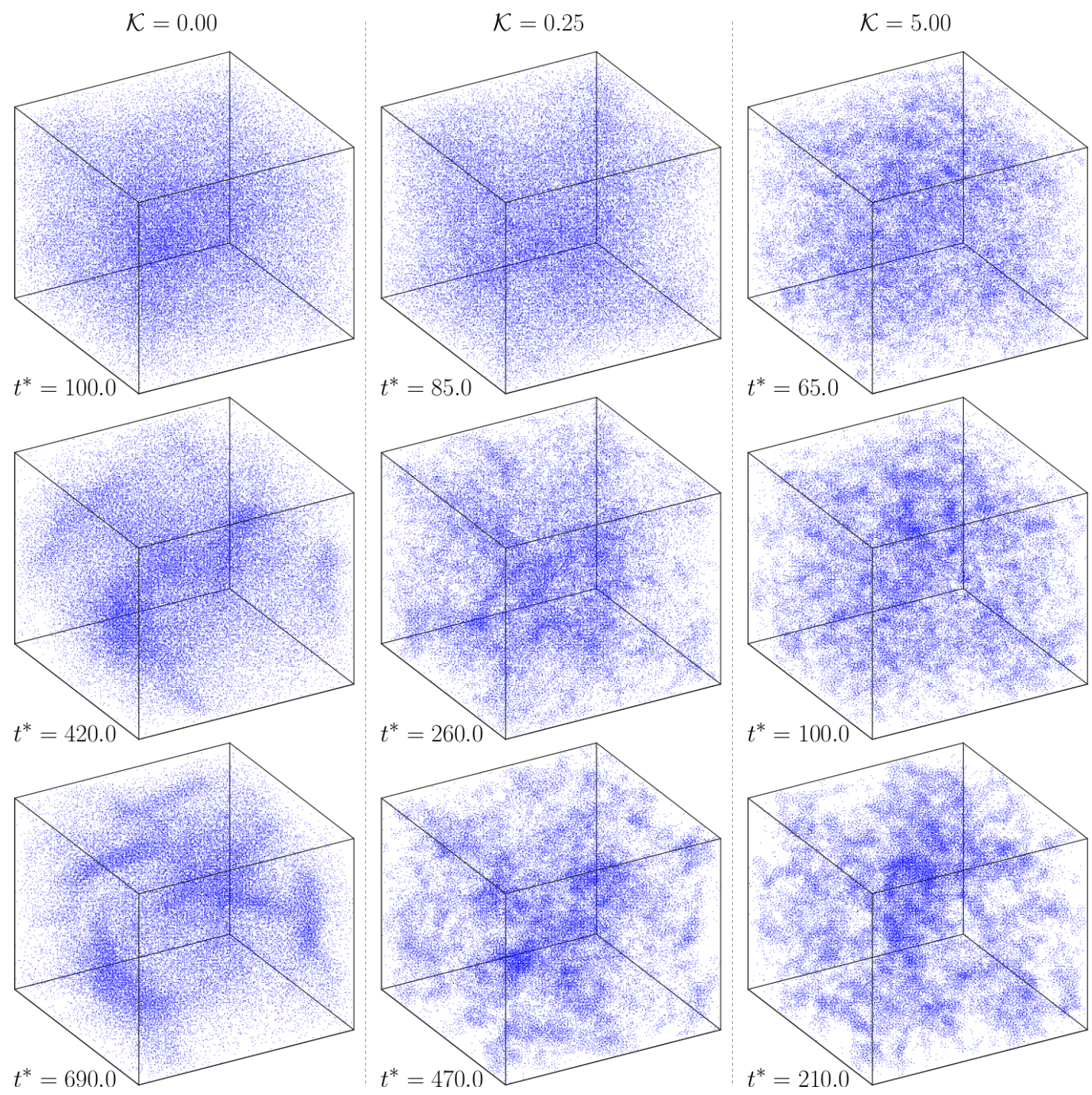

Figure 4.2: Snapshots of the granular gas showing the time evolution of the neutral system of viscoelastic particles (left column) and charged viscoelastic particles (center and right columns). Here time $t^{*}=t v_{\text {ref }} / d$, total number of monomers $N_{\text {mon }}=50016$ and the particle filling fraction in the system $\phi=0.076$. As the ratio of characteristic Coulomb to thermal energy $\mathcal{K}$ increases, the characteristic time for the emergence of clustering decreases, however, their growth rate is unchanged (see also Figs. 4.3 and 4.5). The clusters exhibit a relatively compact morphology in the charged system. 


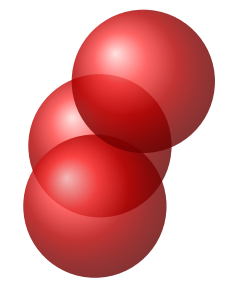

particle $a, t^{*}=70.0$

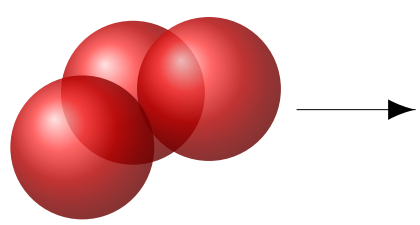

particle $b, t^{*}=120.0$

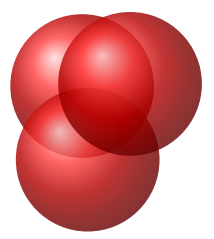

particle $c, t^{*}=50.0$

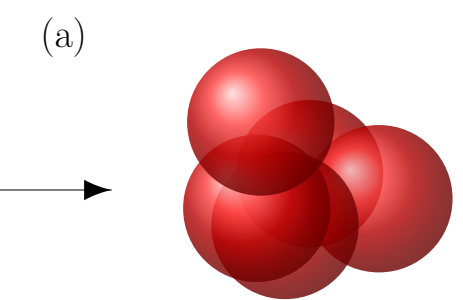

particle $a, t^{*}=410.0$

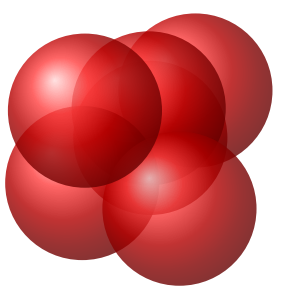

particle $b, t^{*}=410.0$

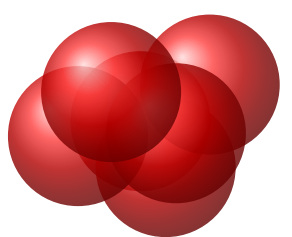

particle $c, t^{*}=410.0$
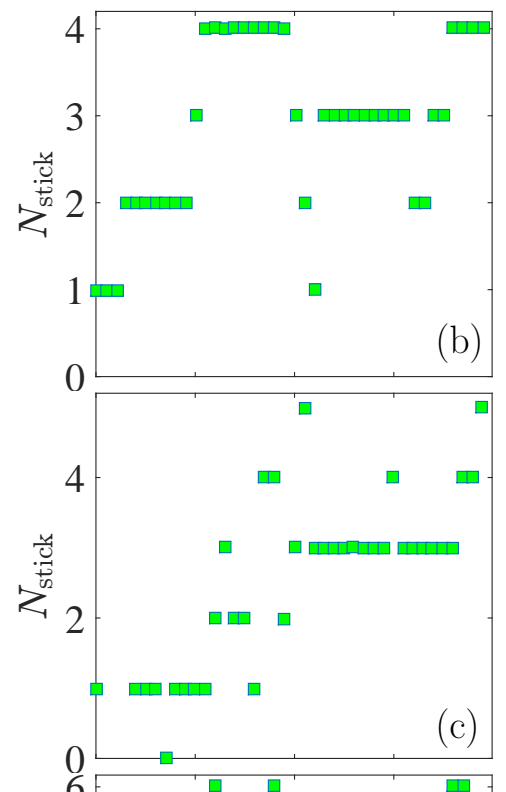

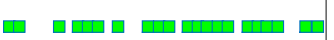

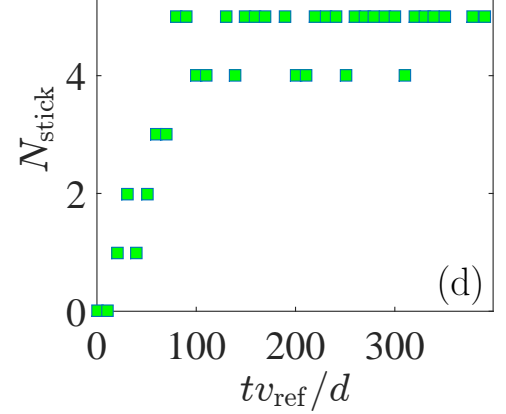

Figure 4.3: Sticking of particles during clustering in the charged system. We observe a mechanism similar to collide-and-capture events observed in experiments on a falling granular stream by Lee et al. (2015). Particles stick together in clusters and exhibit pronounced persistence in each cluster over a considerable duration of time. This mechanism is not observed in the neutral system, where instead particles collide and separate. (a) Specific particles and their first neighbors are shown at different times. (b)-(d) The evolution of the number of contacts, $N_{\text {stick }}$, with time for the same particles shown in panel (a). The occasional fragmentation results in the fluctuation of $N_{\text {stick }}$ with time. 


\subsection{Initialization}

The simulations are initialized by randomly placing the particles in a 3D domain with initial velocities distributed according to a Gaussian with zero mean and $\sqrt{T(0)}$ standard deviation. The (non-dimensional) initial granular temperature $T(0)=10$. Any remaining, initial net velocity of the system $\sum_{i=1}^{N} \mathbf{v}_{i}$ is removed from each particle's velocity $\mathbf{v}_{i}$ to ensure that no net macroscopic flux is present in the system. Initially, all particles have zero charges. We also ensure that the system is at all times globally neutral, i.e. $\sum_{i=1}^{N} q_{i}=0$, by enforcing conservation of charge during each collision event. We let the system equilibrate for some time only under the influence of elastic forces. The dissipation and collisional charging are then switched on.

\subsection{Results and discussion}

The dynamics of granular gases in the absence of electrostatics are reasonably well understood (Nie et al. , 2002; Brilliantov \& Pöschel, 2010; Grossman et al. , 1997; van der Weele et al. , 2001; Mikkelsen et al. , 2002), and exhibit numerous intriguing features such as universal Gaussian velocity distributions in the long-time limit (Nie et al. , 2002), multiscaling and self-similarity in collisions (Ben-Naim \& Krapivsky, 2000; Ben-Avraham et al. , 2003), non-equilibrium steady states and asymmetric velocity distributions under energy inputs (Grossman et al. , 1997), anomalous diffusion (Bodrova et al. , 2016; Brilliantov \& Pöschel, 2000a), ballistic aggregation of clusters as a whole (Paul \& Das, 2017), and dissimilarity between ensemble-averages and long-time averages of observables (non-ergodicity) (Bodrova et al. , 2015). In addition, it is now known from the studies of Takada et al. (2017); Scheffler \& Wolf (2002); Müller (2008) that if a granular gas is composed of equally charged particles (that is, the charge on each particle is equal in sign and magnitude), the number of collisions per unit time decreases due to the Coulomb repulsions. This, in comparison to a neutral granular gas, results in a slower decay rate of the kinetic energy per particle or the granular temperature $T$ as the time progresses. This feature is recovered in our simulations as depicted in Fig. 4.1(a), which shows the decay of $T$ with time for constant coefficient of restitution. At short times, the granular temperature follows Haff's law $\left[T(t) \sim t^{-2}\right]$, however, at later times it deviates from it and 
approaches a slower, inverse logarithmic scaling as was shown analytically by Scheffler \& Wolf (2002). Moreover, the HCS becomes unstable due to dissipative cooling of the granular gas, and clustering emerges. Here we show that the additional perturbations due to collisional charging alter the geometrical morphology of clusters and their growth in time.

Figure 4.2 shows the time snapshots of the system for both neutral $(\mathcal{K}=0.0)$ and charged viscoelastic granular gas $(\mathcal{K}=0.25$ and 5.0$)$. The clusters are relatively elongated for neutral viscoelastic systems, while they are relatively compact in the collisionally charged system. The clustering for non-zero $\mathcal{K}$ initiates through mutual sticking of charged particles and results in the formation of very localized agglomerates of particles [Fig. 4.3]. This agglomeration process is identified by following the trajectories of particles and, via nearest-neighbor search, identifying the particles which are in contact with the followed particle. A contact is defined if $\left|\mathbf{r}_{i j}\right| \leq d_{0}$. As the time progresses, we see that there is a definite trend of particles to stick together [see Fig. 4.3(a)], and the persistence of individual particles in these localized aggregates is rather long-lived [Fig. 4.3(b-d)]. The long persistence of particle contacts is not present in the ICS of the neutral granular gas where particles aggregate due to a mechanism described as a hydrodynamic instability (Brilliantov \& Pöschel, 2010). In fact, a collide-and-capture mechanism has been observed experimentally by Lee et al. (2015), for collisional charging in a falling dilute granular stream. In the experiments (Lee et al. , 2015), the particles collide, bounce multiple times and then tend to stick together giving rise to local aggregates. One particular observation made by Lee et al. (2015) is that when a single particle hits a cluster, it can either get trapped in the electrostatic field or can cause other particles to leave the cluster leading to fragmentation. In our simulations, the fragmentation is observed occasionally as suggested by fluctuating neighbor contacts $N_{\text {stick }}$ in [Fig. 4.3(b-d)] over time.

Figure 4.4(a) shows the time evolution of the mean absolute charge, $\bar{q}=\frac{1}{N} \sum_{i=1}^{N}\left|q_{i}\right|$, in the system. According to our ansatz, the rate and extent of collisional charging strongly depend on the number of collisions occurring per unit time, as well as on the relative velocities between the colliding particles. Due to dissipation, on the other hand, the kinetic energies of the particles keep decreasing and thus effectively contributing to the reduction of charge exchange between the particles. Once the kinetic energy per particle becomes 
sufficiently low, the mean charge in the system begins to saturate. The initial evolution of the mean charge can be estimated by the product of the initial collision frequency $\nu$ and the charge exchange during single collision $\Delta q_{i j}$. Since initially all particles are neutral, the collision frequency must coincide with its neutral counterpart $\nu(t=0)=n \pi b^{2}\left\langle\dot{\xi}_{i j}\right\rangle$ (Scheffler \& Wolf, 2002), where $\left\langle\dot{\xi}_{i j}\right\rangle$ is the mean relative velocity between colliding particles, $n$ is the number density and $b=d_{0}$ is the impact parameter. Then the initial rate of collisional charge exchange is

$$
\nu\left\langle\Delta q_{i j}\right\rangle=n \pi b^{2}\left\langle\dot{\xi}_{i j}\right\rangle\left[C m^{\mathrm{eff}}\left\langle\dot{\xi}_{i j}^{2}\right\rangle\right]^{\eta} e
$$

which is proportional to the rate of mean collisional charging $\dot{\bar{q}}$. Assuming initially a Gaussian velocity distribution, so that $\left\langle\dot{\xi}_{i j}\right\rangle \propto T^{1 / 2}$, and considering the fact that for viscoelastic particles $T \propto t^{-5 / 3}$, the evolution of $\bar{q}$ obeys

$$
\frac{d \bar{q}}{d t} \propto T^{1 / 2} T^{\eta} \propto t^{-5 / 6} t^{-5 /(3 \eta)}
$$

As experiments show that $\eta \approx 0.8$ (Poppe et al. ,2000b), the rate of mean charge buildup with time is then

$$
\frac{d \bar{q}}{d t} \propto t^{-13 / 6}
$$

A fit of the simulation results to Eq. (4.6) is also shown in Fig. 4.4(a), which closely follows the initial charge buildup. However, later in time it deviates from the prediction in equation Eq. (4.6) indicating that the collision rate or the relative velocities of impact between particles after charge buildup are suppressed. This is expected after mutual sticking of particles.

The conservation of charge at a single collision level and the initial condition $\sum_{i=1}^{N} q_{i}=0$ results in the fact that there is a statistically equal number of pairwise attractions and repulsions. This symmetry of the sign of charge among mono-dispersed particles has also been shown recently through experiments for a global charge conserved system, and when no other material or wall is present (Lee et al. ,2015). As a consequence of this symmetric charge distribution, the early evolution of the granular temperature does not show any considerable deviation from the Haff's law [Fig.4.1(b)]. On the other hand, if the number of pairwise repulsive interactions exceed the attractive, the rate of the decay 


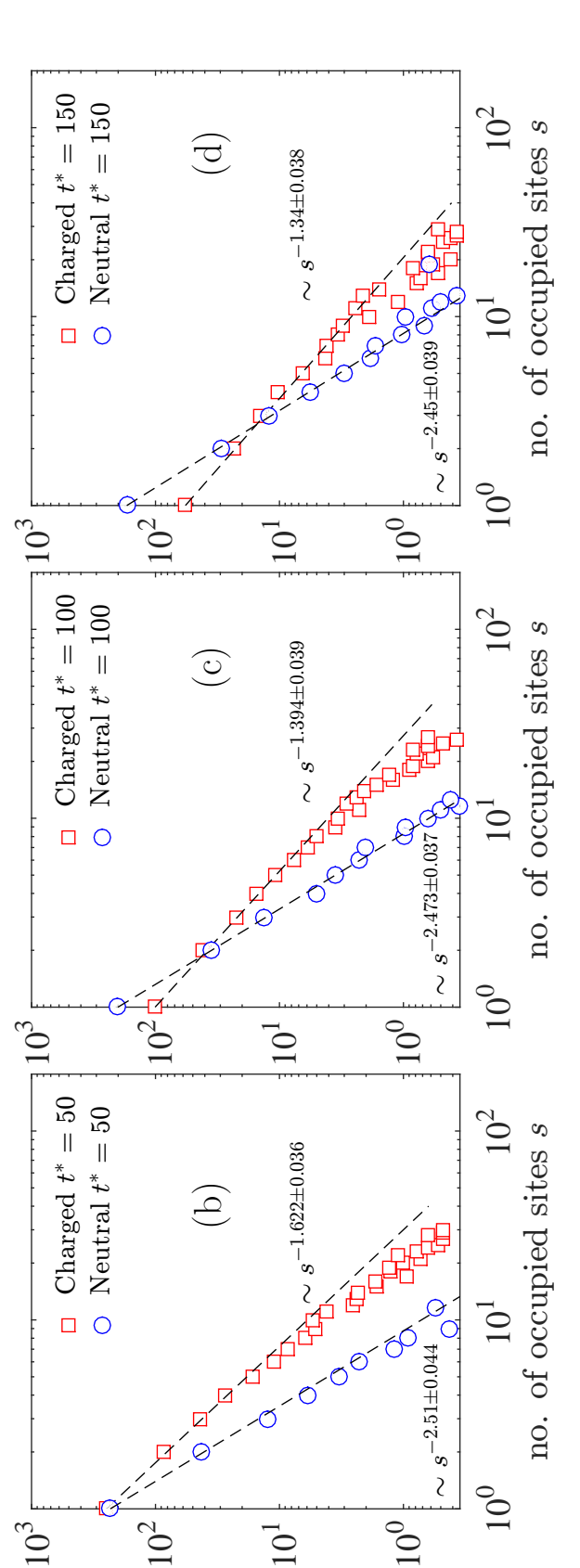

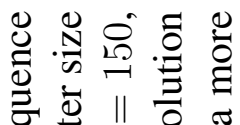

要

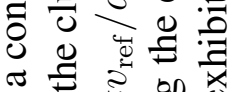

政要

告

密

寻苛

की हैं

공

造至要

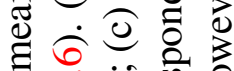

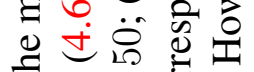

F $\|$ ठ

$\dot{0} \Xi \approx 0$

0

घ.

㱐 $\hat{e}$

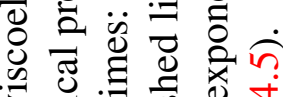

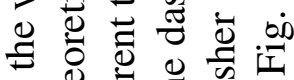

o

. 0 \%

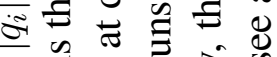

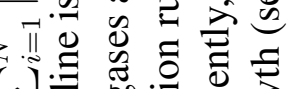

W:

$-\mid z:=$ 흘

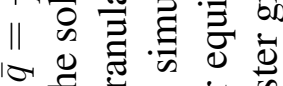

of

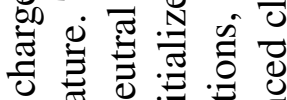

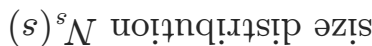

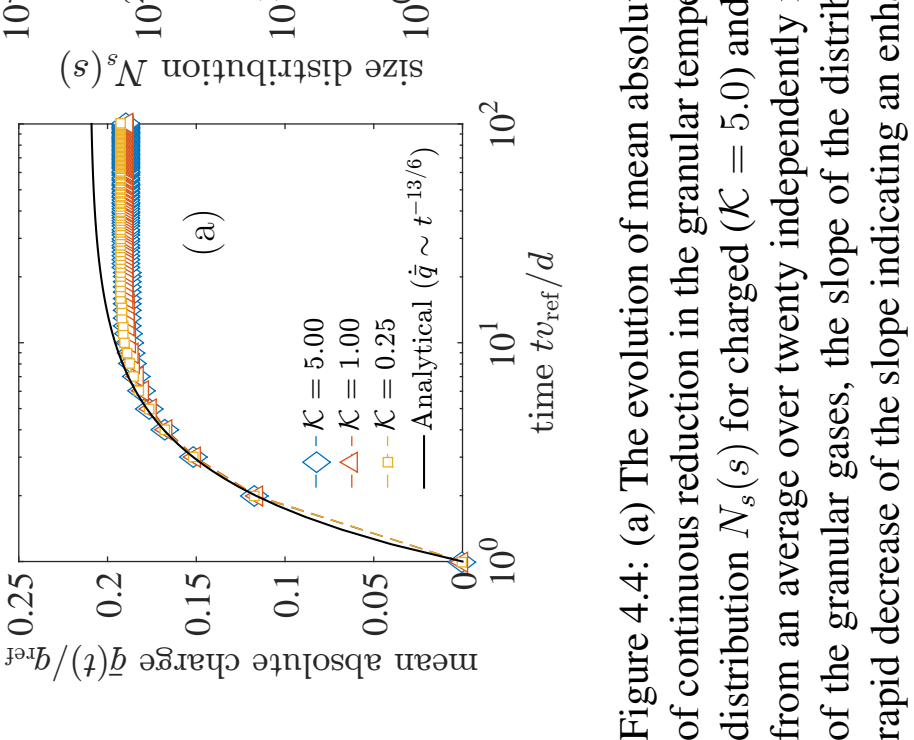


of $T$ slows down, as evident in Fig. 4.1(a).

\subsubsection{Clustering}

To investigate the statistical properties of the clusters, we calculate the cluster size distribution. A time-dependent matrix which contains information about the occupied (or dense) and unoccupied (or dilute) sites in the system is obtained by thresholding the coarse-grained particle density in the system (Paul \& Das, 2017). Specifically, we divide the simulation domain into equal-size boxes and, at any given time, compute the particle density in each of the boxes. The boxes which have particle density higher than a threshold value is then labeled as an 'occupied site' and the rest of the boxes as 'unoccupied' ${ }^{1}$. A cluster is then defined as the region of such connected occupied sites. This definition of clusters provides the advantage that it is independent of whether granular particles are in contact with each other in a strict sense (as they are in the charged case) or they only form density inhomogeneities without contacts (as in neutral granular gases). The size distribution $N_{s}(s)$ of such connected occupied sites is then obtained. The size distribution asymptotically scales as

$$
N_{s}(s) \sim s^{-\tau}
$$

where $\tau$ is so called the Fisher exponent (Stauffer, 1979; Stauffer \& Aharony, 2003). Figure 4.4(b-d) shows $N_{s}(s)$ for both the neutral and the charged scenarios. The Fisher exponent during early aggregation increases relatively quickly in the charged case (from -1.62 to -1.34 ) compared to the neutral system (from -2.51 to -2.45 ). Additionally, the size distribution of the occupied sites is relatively broader for the charged gas. The count for a given cluster size $s$ is larger in the charged system, except for very small $s$. This suggests, in relation to the observations in Fig. 4.2, that the clusters in the charged gas are compact and more numerous.

This difference in the rate of change of Fisher exponent in the charged system results

\footnotetext{
${ }^{1}$ The threshold filling fraction is taken equal to a third of the maximum possible filling fraction, that is, the hexagonal close packing for equal spheres, $\phi_{\mathrm{hcp}}=\frac{\pi}{3 \sqrt{2}}=0.7405$. Changing the threshold in our calculations only results in a different multiplicative factor for the average cluster size, and does not change the growth exponents.
} 
in a different growth exponent of the so called average cluster size

$$
S(t)=\frac{\sum_{s} s^{2} N_{s}}{\sum_{s} s N_{s}} .
$$

For the neutral gas, a best fit to the average over twenty independently initialized simulations reveals [Fig. 4.5]

$$
S(t) \sim\left[t^{1.21 \pm 0.04} \approx t^{6 / 5}\right]
$$

which is close to the mean field result based on the Smoluchowski's aggregation equation (Leyvraz, 2003; Pathak et al. , 2014). The error in the exponent represents a 95\% confidence level. For the charged gas, we obtain

$$
S(t) \sim\left[t^{1.49 \pm 0.012} \approx t^{3 / 2}\right]
$$

which clearly indicates a relatively faster cluster growth [Fig. 4.5]. The growth exponent $z$ can be precisely obtained using the method of local slope

$$
z=\frac{\log [S(t) / S(t / p)]}{\log (p)}
$$

where $p$ characterizes the time resolution (Lübeck, 2004). In the limit $1 / t \rightarrow 0$, the function should attain a saturation value, which is the best estimate for $z$. Fig. 4.5 (inset) shows this saturation of $z$ to $\approx 1.5$ for the charged while to $\approx 1.21$ for the neutral gas as $t$ increases (or $1 / t \rightarrow 0$ ).

For early stages of growth, in the charged system, the cluster growth exponent $z$, remarkably, does not show a dependence on the ratio of the characteristic Coulomb to thermal energy $\mathcal{K}$ or equivalently on the typical dimensionless Bjerrum length $\ell_{\mathrm{B}} / d_{0}$. In Fig. 4.5 we show $S(t)$ for increasing $\mathcal{K}=0.25,1.0$ and $\mathcal{K}=5.0$. Upon decreasing $\mathcal{K}$, the characteristic time $t_{c}$ for the emergence of aggregation increases and approaches the neutral case as $\mathcal{K} \rightarrow 0$. However, once the aggregation starts, it does not influence the growth exponent $z$. An increase of $t_{c}$ upon decreasing $\mathcal{K}$ is reminiscent of the selffocusing Brownian aggregation of monopolarly charged particles found by Dammer \& Wolf (2004). 


\subsubsection{Mean-field: Smoluchowski aggregation equation with modified reaction kernel}

Aggregation phenomena are very common in nature, for example, the coalescence of raindrops, coagulation of snow, aerosol, and powders, polymerization, or agglomeration in colloids. One is often interested in determining, at a given time during the aggregation process, the sizes of the aggregates and the rate at which they grow in time. A simple way to approach is via a mean-field theory, which has been extensively applied (mainly for uncharged particle systems) to precisely predict the time-dependent aggregate size distributions and their growth rate. In this, only simplified features of the underlying transport processes and the nature of the physical interactions between the aggregates are taken into account. Here we make a mean-field approximation of the aggregation of the initially monodisperse system using the discrete form of the Smoluchowski's equation [Eq. (2.50)] derived in chapter 2, i.e.

$$
\begin{array}{r}
\frac{\partial f\left(N_{i}, t\right)}{\partial t}=\frac{1}{2} \sum_{j=1}^{i-1} K_{i-j, j} f\left(N_{i}-N_{j}, t\right) f\left(N_{j}, t\right) \\
-f\left(N_{i}, t\right) \sum_{j=1}^{\infty} K_{i, j} f\left(N_{j}, t\right),
\end{array}
$$

where $f\left(N_{i}, t\right)$ is the number density of aggregates having $N_{i}$ monomers at a time $t$, and $K_{i, j}$ is the aggregation kernel [Eq. (2.46)]. The kernel $K_{i, j}$ is typically related to the collision cross-section $\sigma_{i j}$ of the colliding aggregates and the relative aggregate velocity $v_{i j}$ as

$$
K_{i, j} \propto \sigma_{i j} v_{i j}
$$

The collision-cross section is dependent on the aggregate size while the velocity part of the kernel is related to the diffusion of the aggregates. Numerical solution of Eq.(4.12) with a well-known kernel of the form

$$
K_{i, j} \propto\left(N_{i}^{1 / 3}+N_{j}^{1 / 3}\right)^{2}\left(N_{i}^{-1}+N_{j}^{-1}\right)^{1 / 2}
$$

from the kinetic theory of uncharged particle aggregation (Antony et al. , 2004) yields a growth exponent $z=1.19$ which is close to our result for the neutral case in Fig. 4.5. 
Electrostatic interactions are difficult to treat as they result in an additional dependence of $K_{i, j}$ on the charge distribution among the aggregates besides the dependence on the size distribution. It is, however, possible to include the effects of electrostatic interactions in the aggregation kernel (Dammer \& Wolf, 2004; Ivlev et al. , 2002). If the charge on the individual particles is time-dependent and fluctuating, as in the present study, even the solution of the above mean-field theory becomes quite complex. As a first attempt for a collisionally charged granular gas, here we indirectly introduce the electrostatic effects in the Smoluchowski's kernel $K_{i, j}$ based on the results from the MD simulations. Under this, for a bipolarly charged gas, we argue that the average collision cross-section $\left\langle\sigma_{i j}\right\rangle$ remains statistically unchanged due to the symmetry of the charge distribution. ${ }^{1}$ This should be true as long as the net charge in the system is zero. This conjecture is consistent with the result in Fig. 4.1(b), which shows no significant deviation of $T$ from the neutral system, at least in the early stage of evolution. However, the relative aggregate velocities $v_{i j}$ are expected to be suppressed due to the mutual sticking of particles and their entrapment in the electrostatic field, as discussed previously. This fact is modeled in the kernel by introducing a variable $\beta$ as

$$
K_{i, j} \propto\left(N_{i}^{1 / 3}+N_{j}^{1 / 3}\right)^{2}\left(N_{i}^{-1}+N_{j}^{-1}\right)^{1 / \beta},
$$

where $\beta=2$ corresponds to the neutral aggregation. An increasing $\beta$ simply implies a suppressed diffusion. We numerically solve the Smoluchowski's equation with the kernel (4.15). The growth exponent $z \rightarrow 1.49$ when $\beta \rightarrow 3$. The increase in the value of $z$ in the mean-field theory when the velocity term $v_{i j}$ is suppressed supports the argument of reduction in the relative aggregate velocities.

To support the inclusion of the parameter $\beta$ in the diffusion part of the kernel, we study the mean square displacement (MSD) of the particles. This is depicted in Fig. 4.6. The MSD in a dissipative granular gas is known to exhibit a sub-diffusive behavior (Bodrova et al. , 2016). Figure 4.6 (inset) shows that the sub-diffusive regime due to dissipation is further suppressed due to the electrostatic interactions. The fact that the MSD is strongly sub-diffusive is consistent with a reduced relative aggregate velocity, and thus with in-

\footnotetext{
${ }^{1}$ The impact-parameter for individual collisions will be different depending on the charge magnitude and sign on the particles, however, here it is assumed that the average number of attractive encounters in the gas are equal to the average number of repulsive encounters.
} 


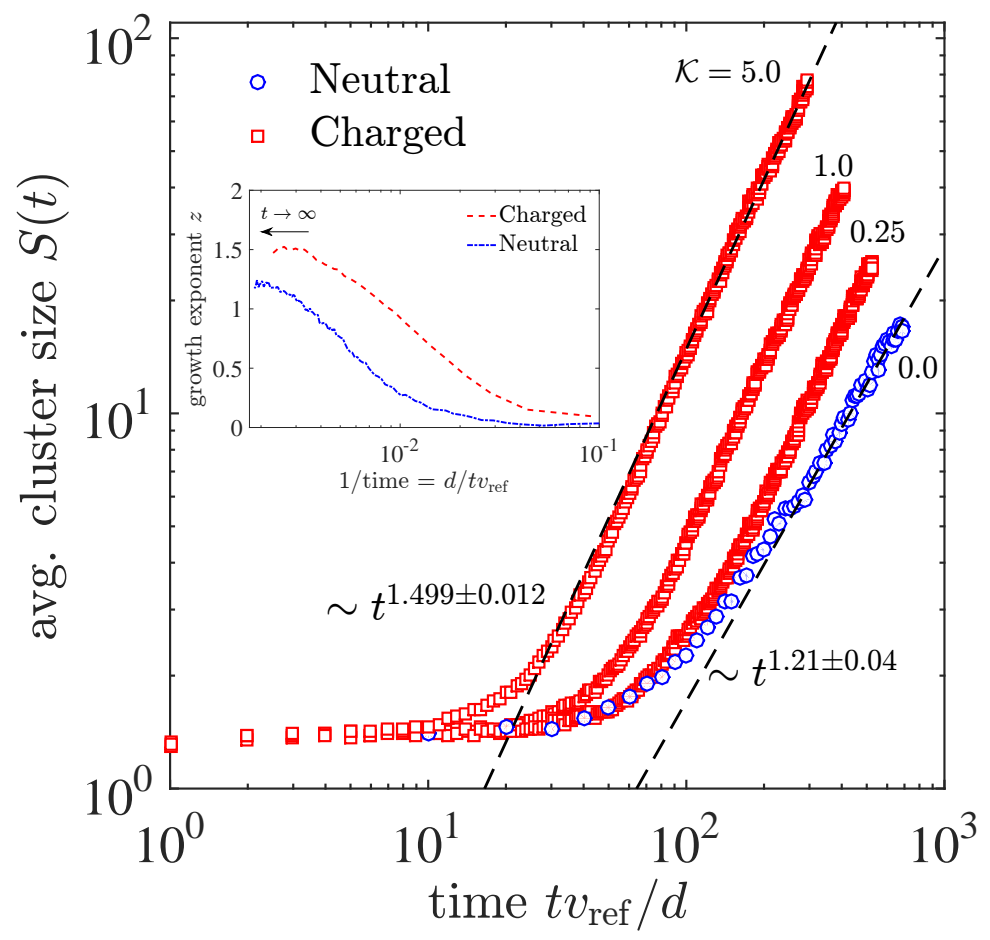

Figure 4.5: During early stages of the growth, the temporal dependence of the average cluster size $S(t)$, from an average over twenty independently initialized simulation runs and corresponding best fit. Granular gas with collisional charging exhibit faster growth of clusters than for neutral system. (Inset) saturation of the growth exponent $z$ as $1 / t \rightarrow$ 0 using the method of local slope [Eq. (4.11)]. A change in the ratio of characteristic Coulomb to thermal energy $\mathcal{K}$ do not alter the growth exponent $z$ and only influences the crossover time of initiation of clustering. 


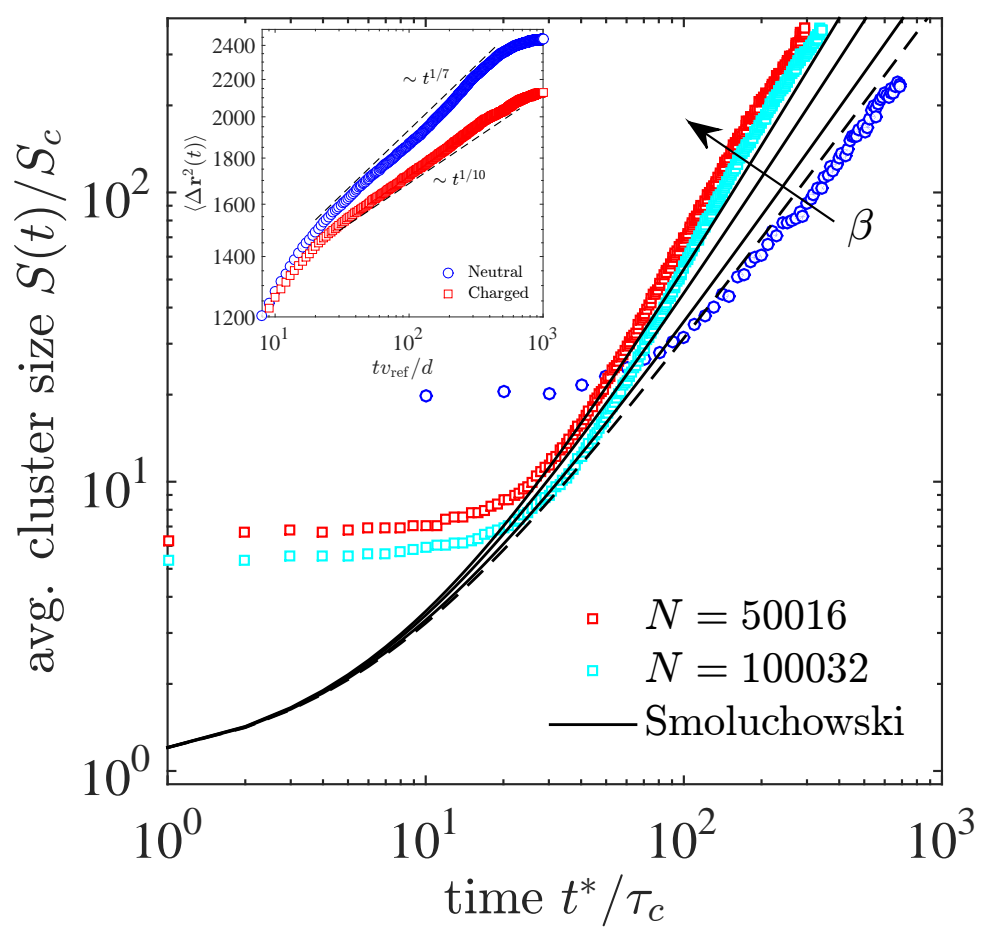

Figure 4.6: Comparison of the average cluster size $S(t)$ predicted by the Smoluchowski equation with the MD results. At $\beta=2$ (dashed line) the mean-field solution agrees reasonably well with the neutral gas, while at $\beta=3$ (solid line) the growth rate for the charged granular gas is recovered. (Inset) Comparison of the MSD of particles between the charged and the neutral gas. The sub-diffusion due to dissipation is further suppressed by the electrostatics, which in the mean-field approximation is modeled by increasing $\beta$. Here $S_{c}$ and $\tau_{c}$ are factors used to rescale the curves and plot them close to each other for the sake of comparison of the slopes. Here the non-dimensional time $t^{*}=t v_{\text {ref }} / d$. 
creasing $\beta$.

The Smoluchowski's equation can be further simplified, if a strict monodisperse mass distribution of the aggregates is assumed at any time $t$, i.e. at a given time $t$, only aggregates with size $N_{i}\left(N_{i}\right.$ are the number of smallest possible entities, for example number of monomers or the number of the occupied sites, in the $i_{t h}$ aggregates) are present and the number density of aggregates of size $N_{j}$ other than $N_{i}$ is zero. This approximation is rather severe, however it reduces the Smoluchowski's equation to an analytically solvable form, written as (Blum, 2006)

$$
\frac{\partial f\left(N_{i}, t\right)}{\partial t}=-K_{i, i} f^{2}\left(N_{i}, t\right)
$$

where the kernel $K_{i, j}$ now takes the following form

$$
K_{i, i} \propto\left(N_{i}^{1 / 3}\right)^{2}\left(N_{i}^{-1}\right)^{1 / \beta}=N_{i}^{1 / 6} N_{i}^{-1 / \beta}
$$

If the total mass in the system is conserved, then $f\left(N_{i}, t\right) N_{i}(t)=$ const., and one can transform Eq. (4.16) to the following

$$
\frac{\partial N_{i}(t)}{\partial t}=\frac{1}{\tau_{0}}\left(N_{i}^{1 / 6} N_{i}^{-1 / \beta}\right)
$$

where $\tau_{0}$ is some characteristic time. The solution of this ordinary, but non-linear differential equation is

$$
N_{i}(t) \sim t^{z}, \text { with } \begin{cases}z=6 / 5, & \text { if } \beta=2, \\ z>6 / 5, & \text { if } \beta>2, \\ z=3 / 2, & \text { if } \beta=3 .\end{cases}
$$

Figure 4.6 also shows the comparison of Eq. (4.19) with the MD results. Upon changing $\beta$ from 2 to 3 the mean-field calculations agree reasonably well with the MD results, which is consistent with the suppression of diffusivity due to electrostatics [Fig. 4.6 (inset]. We find that the system size does not affect our results. Above it is considered that the number of monomers in the $i^{\text {th }}$ aggregate, i.e $N_{i}(t)$, scales linearly with the average size of the occupied sites $S(t)$. The consistency of the results from numerically solving the kernel in Eq. (4.15), MD calculations, and Eq. (4.19) suggest that the suppression of particle 
diffusion due to electrostatics enhances the aggregation process.

\subsubsection{Velocity distribution}

To analyze the global effects of charging on the dynamical state of the system, we also study the evolution of the normalized velocity distribution function $f(\tilde{v})$, where $\tilde{v}=v /\left\langle v^{2}\right\rangle^{1 / 2}$, and its deviation from the equilibrium, Maxwellian distribution $f_{\mathrm{MB}}(\tilde{v})$. For viscoelastic particles $f(\tilde{v})$ quickly deviates from the Maxwell distribution early in time, attains a maximal deviation regime and tends to approach back the Maxwellian (Brilliantov \& Pöschel, 2010). This behavior for neutral viscoelastic particles is shown in Fig. 4.7(a), where the relaxation of $f(\tilde{v})$ after its maximal deviation is highlighted. The physical reasoning behind this relaxation is that as $t \rightarrow \infty$, the impact velocities $\left(\mathbf{v}_{i j} \cdot \mathbf{n}_{i j}\right)$ tend to reduce which implies the coefficient of restitution $\epsilon=-\mathbf{v}_{i j}^{\prime} \cdot \mathbf{n}_{i j} / \mathbf{v}_{i j} \cdot \mathbf{n}_{i j} \rightarrow 1$ (Here $\mathbf{v}_{i j}^{\prime}$ is the post collision relative velocity). This causes most collisions to be effectively elastic and thus $f(\tilde{v}, t) \rightarrow f_{\mathrm{MB}}(\tilde{v})$ (Pöschel et al. , 2003). The intriguing finding in our study is that the deviation of $f(\tilde{v})$ from the Maxwellian is more significant in dynamically charged systems and it does not exhibit a relaxation towards $f_{\mathrm{MB}}(\tilde{v})$ within the early stage of aggregation. Figure $4.7($ b) shows $f(\tilde{v})$ at different times for a charged system. The deviation of $f_{\mathrm{MB}}(\tilde{v})$ from $f(\tilde{v})$ is more pronounced in the charged case than in the neutral gas and indicates that the nature of the clustering is different in case of dynamically charged systems as compared to the neutral system. The tail of the distribution nearly scales as $f(\tilde{v}) \sim \exp (-\tilde{v})$ in both cases. Over time, the most probable value of $\tilde{v}$ is reduced in the charged system [Fig. 4.7 (b) (inset)]. On the other hand, this deviation is minimal for the neutral gas particles [Fig. 4.7 (a) (inset)]. The difference between the two cases again indicates a reduced motility of particles in the charged gas.

The high-speed particles from the tail of $f(\tilde{v})$ compensate for the reduction of the most probable velocity. To understand which particles are-statistically-responsible for the fat exponential tail of the distribution, we consider a scatter plot of charge and speed for our system, as shown in Fig. 4.8. As the time elapses, a subpopulation of weakly charged particles with high velocities can be identified. This subpopulation corresponding to the fat tail of the distribution suggests an interesting and counterintuitive fact. Although the tail of the distribution deviates significantly from $f_{\mathrm{MB}}(\tilde{v})$ in the charged systems, weakly 

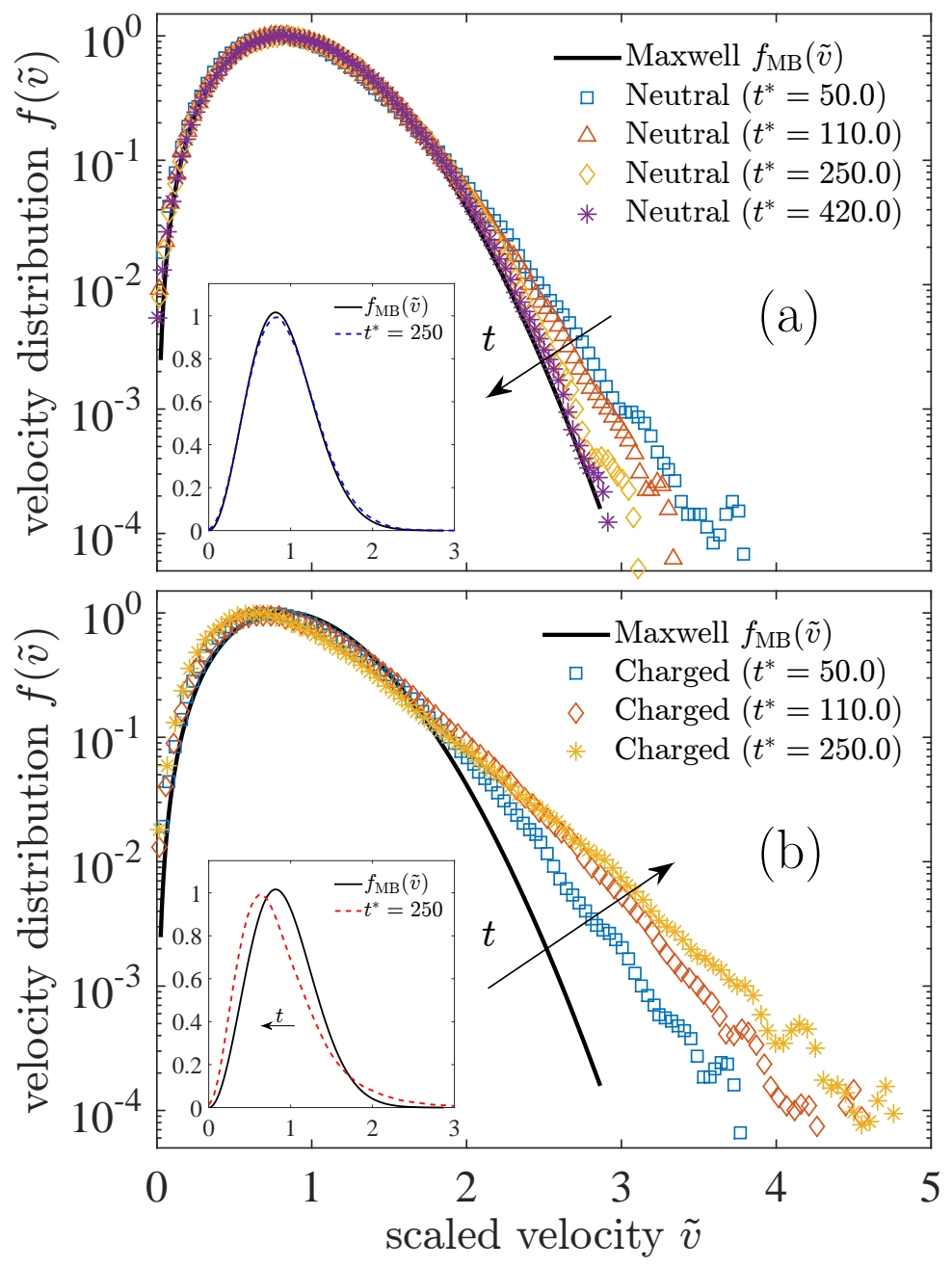

Figure 4.7: (a) The relaxation of the scaled velocity $\left(\tilde{v}=v /\left\langle v^{2}\right\rangle^{1 / 2}\right)$ distribution function towards the Maxwellian for neutral viscoelastic particles. This result from our simulations for neutral viscoelastic particles is consistent with the Sonine expansion for the time dependent distribution function (Brilliantov \& Pöschel, 2010) which depicts that the distribution relaxes back towards the Maxwellian for long time. (b) The time evolution of the distribution $f(\tilde{v})$ for a charged system, however, shows a behavior opposite to the neutral case: the distribution does not relax back to the Maxwellian. (Insets) Same results as in (a) and (b) but on a linear scale to highlight the shift of the most probable velocity for the charged granular gas. Here the non-dimensional time $t^{*}=t v_{\text {ref }} / d$. 

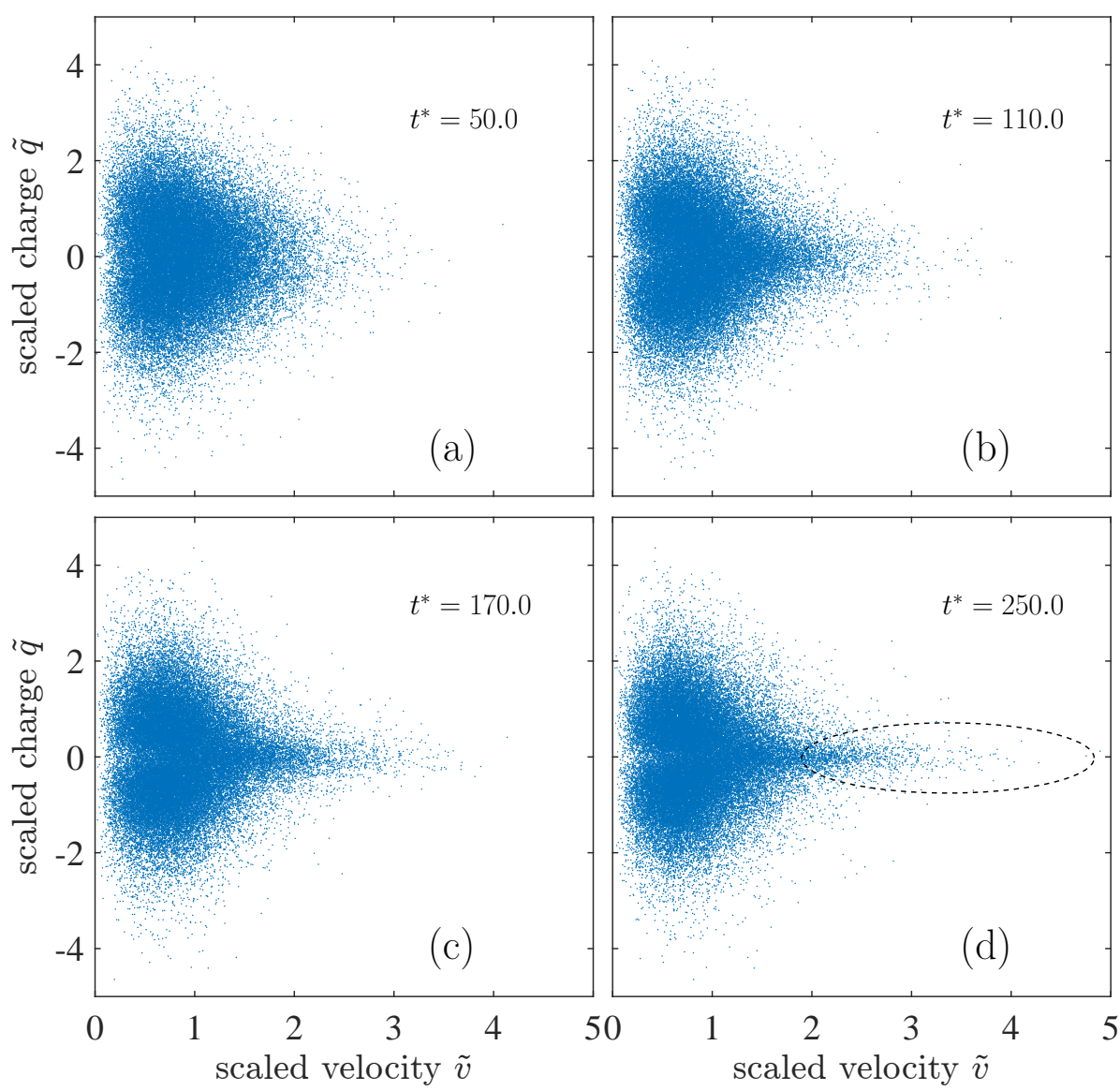

Figure 4.8: Scatter plot of the particles' charges and velocities at different times. As time progresses, a subpopulation of high velocity, nearly neutral particles develops (highlighted in the dashed ellipse). This subpopulation is expected to cause the occasional fragmentation of the local aggregates (see Fig. 4.3). Here $\tilde{q}=q /\left\langle q^{2}\right\rangle^{1 / 2}$ and $\tilde{v}=v /\left\langle v^{2}\right\rangle^{1 / 2}$. Here the non-dimensional time $t^{*}=t v_{\text {ref }} / d$. 
charged particles are actually responsible for it. These weakly charged and high-speed particles fail to stick and form agglomerates. It can be imagined that these particles experience fewer collisions due to the fact that more space is provided by the agglomeration process. Thus the nonequilibrium nature of the charged granular gas is enhanced indirectly through the agglomerating particles, and directly by the weakly charged, high-speed particles. This scenario implies a thermal decoupling between highly charged particles and the subpopulation of weakly charged particles. We expect that due to their relatively high velocities, these weakly charged particles are then the most probable reason behind the occasional fragmentation of the local aggregates, as observed in Fig. 4.3. In neutral freely-cooling granular gases, the exponential tails of the velocity distribution during the intermediate time regime are dominated by the fastest moving particles which manage to avoid any collision with other particles (Nie et al. , 2002). However, the number of such particles decreases over time and eventually the large-velocity tail tends to diminish (Nie et al. , 2002). In the present scenario, the "survival probability" of such uncharged fastestmoving particles is expected to be higher as they gain more accessible volume due to the compact coagulation of the other charged particles. Consequently, the large-velocity tail survives, at least during the early stages of the particle aggregation.

\subsection{Conclusions}

We have studied the effect of collisional charging on the aggregation dynamics of dilute, freely cooling granular gases of viscoelastic particles. We perform molecular dynamics simulations that take into account the collisional charge exchange, and the electrostatic interactions by means of the Ewald summation. Our simulations depict that the electrostatic interactions due to collisional charging alter the morphology ${ }^{1}$ [Fig. 5.1] and the growth rate of the clusters. In a charged system, the local sticking of particles triggers the aggregation, and the subsequent growth of the average cluster size is enhanced [Fig. 4.5 and 4.6]. The growth of the average cluster size is found to be independent of the ratio of characteristic Coulomb to thermal energy, or equivalently, of the typical Bjerrum length. The combined results from the numerical solution of Eq. (4.12) with the modified reaction kernel in Eq. (4.15), the results in Eq. (4.19), the behavior of $f(\tilde{v})$ as shown in Fig. 4.7

\footnotetext{
${ }^{1}$ The morphology of the aggregates is further studied in detail via the fractal dimension in chapter 5.
} 
(b), and the MD results all suggest that electrostatic interactions enhance the aggregation process in a charged granular gas.

In our work, some important physical ingredients such as friction, rotational degrees of freedom of the particles as well as other charging mechanisms such as ionization of the particle interstitial gas due to irradiation, are not included. Especially important is to further improve the charge exchange model [Eq. (4.2), see also Table 3.1]. However, our study, we believe, will be helpful in clarifying very basic feature of natural processes which produce dust aggregation in charged environments $e . g$., the agglomeration of planetary dust (Blum et al. , 2000) and cohesive powder substructures (Wolf et al., 2009). In the perspective of planetary dust aggregation, it will also be interesting to include the effects due to drag caused by interstitial gas surrounding the particles, dipolar effects (Siu et al. , 2015; Wesson, 1973), as well as van der Waals effects (Blum et al. , 2000). 



\section{Chapter 5}

\section{Electrification in charged granular}

\section{gases leads to constrained fractal}

\section{growth $^{1}$}

The empirical observation of aggregation of dielectric particles under the influence of electrostatic forces lies at the origin of the theory of electricity. The growth of clusters formed of small grains underpins a range of phenomena from the early stages of planetesimal formation to aerosols. However, the collective effects of Coulomb forces on the nonequilibrium dynamics and aggregation process in a granular gas - a representative of the above physical processes - have so far evaded theoretical scrutiny. Here, we establish a hydrodynamic description of aggregating granular gases that exchange charges upon collisions and interact via the long-range Coulomb forces. We analytically derive the governing equations for the evolution of granular temperature, charge variance, and number density for homogeneous and quasi-monodisperse aggregation. We find that, once the aggregates are formed, the granular temperature of cluster population, the charge variance of cluster population and the number density of cluster population evolve in such a way that their non-dimensional combination obeys a physical constraint of nearly constant dimensionless ratio of characteristic electrostatic to kinetic energy $\mathcal{B}(t) \leq 1$. This constraint on the collective evolution of charged clusters is confirmed both by the theory and the detailed molecular dynamics simulations. The inhomogeneous aggregation

\footnotetext{
${ }^{1}$ The content of this chapter has been published in: Singh, Chamkor, and Marco G. Mazza. "Electrification in granular gases leads to constrained fractal growth." Scientific reports 9.1 (2019): 9049.
} 
of monomers and clusters in their mutual electrostatic field proceeds in a fractal manner. Our theoretical framework is extendable for more precise charge exchange mechanism, a current focus of extensive experimentation. Furthermore, it illustrates the collective role of long-range interactions in dissipative gases and can lead to novel designing principles in particulate systems. 


\subsection{Introduction}

The electrostatic aggregation of small particles is ubiquitous in nature and ranks among the oldest scientific observations. Caused by collisional or frictional interactions among grains, large amounts of positive and negative charges can be generated. These clusters have far-reaching consequences: from aerosol formation to nanoparticle stabilization (Castellanos, 2005; Schwager et al. , 2008), planetesimal formation, and the dynamics of the interstellar dust (Wesson, 1973; Harper et al. , 2017; Brilliantov et al. , 2015; Blum, 2006). The processes accompanying granular collisions, charge buildup and subsequent charge separation can also lead to catastrophic events such as silo failure, or dust explosions.

Experimental investigations of the effects of tribocharging date back to Faraday, and recent in situ investigations have revealed important results (Jungmann et al. , 2018; Lee et al. , 2015; Yoshimatsu et al. , 2017; Poppe et al. , 2000b; Haeberle et al. , 2018). However, technical difficulties plague even careful experiments and often impede their unambiguous interpretation (Spahn \& Seiß, 2015). A source of these difficulties is the lack of consensus about whether electrostatics facilitate or hinder the aggregation process of a large collection of granular particles (Spahn \& Seiß, 2015). Despite considerable effort (Ivlev et al. , 2002; Dammer \& Wolf, 2004; Müller \& Luding, 2011; Ulrich et al. , 2009; Brilliantov et al. , 2018; Liu \& Hrenya, 2018; Takada et al. , 2017; Kolehmainen et al. , 2018b) a statistico-mechanical description of aggregation in a dissipative granular system with a mechanism of charge transfer is still lacking. The theoretical treatment requires reconsideration of the dissipation of kinetic energy conventionally described by a monotonic dependence of the coefficient of restitution on velocities $\epsilon(v)$, and also the inclusion of long-range electrostatic forces due to the dynamically-changing charge production. Understanding the growth of charged aggregates requires a statistical approach due to the different kinetic properties and aggregate morphology.

In this work, we present a modified Boltzmann description for the inelastic and aggregative collisions of grains that interact via Coulomb forces, and exchange charges upon collision. We derive the hydrodynamic equations for the number density $n$, the granular temperature $T$, and the charge fluctuations $\left\langle\delta q^{2}\right\rangle$ of the aggregates under the assumptions of homogeneous and quasi-monodisperse aggregation. We find that the dimensionless 
ratio of the characteristic electrostatic energy $\frac{k_{e}\left\langle\delta q^{2}\right\rangle}{d}$ to the granular temperature T, i.e. $\frac{k_{e}\left\langle\delta q^{2}\right\rangle}{T d}$, approaches but stays below 1 . Here $k_{e}$ is the Coulomb's constant while $d$ is the characteristic size of the aggregates.

To bolster our results, and explicitly consider fluctuations in dynamics and morphological structures, we also use three-dimensional molecular dynamics (MD) simulations that explicitly include Coulombic interactions and a charge-exchange mechanism. We find that the granular dynamics agree quantitatively with the predictions of the Boltzmann equation. The cooling gas undergoes a transition from a dissipative to an aggregative phase marked by a crossover in the advective transport. We explore the morphological dynamics of the inhomogeneous aggregation via the mean fractal dimension and their interplay with the mesoscopic flow.

\subsection{Kinetics}

In general, agglomeration in a three-dimensional collisionally charging cooling granular gas is a spatially inhomogeneous process which involves the interplay between dissipation, the time-varying size distribution of aggregates, charge fluctuations and exchange mechanism during collisions, long-range forces, and collective effects (Singh \& Mazza, 2018). This complexity is illustrated in Fig. 5.1 which shows snapshots of cooling clusters from a typical MD simulation, beginning from a homogeneous and neutral state (see Methods for MD). In the following, we establish a modified Boltzmann approach for this intricate dynamics of the aggregation process, which predicts novel physical limits.

We consider the single particle probability distribution function $f=f(\mathbf{r}, t ; \mathbf{v}, q, d)$, where the particle velocity $\mathbf{v}$, charge $q$, position $\mathbf{r}$ and particle size $d$, are the phase space variables, and $t$ denotes time. We specialize to a homogeneous and quasi-monodisperse aggregation scenario (i.e. the size is assumed to vary in time but spatially mono-dispersed, see schematic representation in Fig. 5.2). Under these limits, the spatial and particle-size dependence of $f$ drops out, i.e. $f=f(t ; \mathbf{v}, q)$ only, and its time evolution is given by the simplified Boltzmann equation (Pitaevskii \& Lifshitz, 2012; Brilliantov \& Pöschel, 2010)

$$
\frac{\partial f}{\partial t}=I_{\text {coll }}
$$




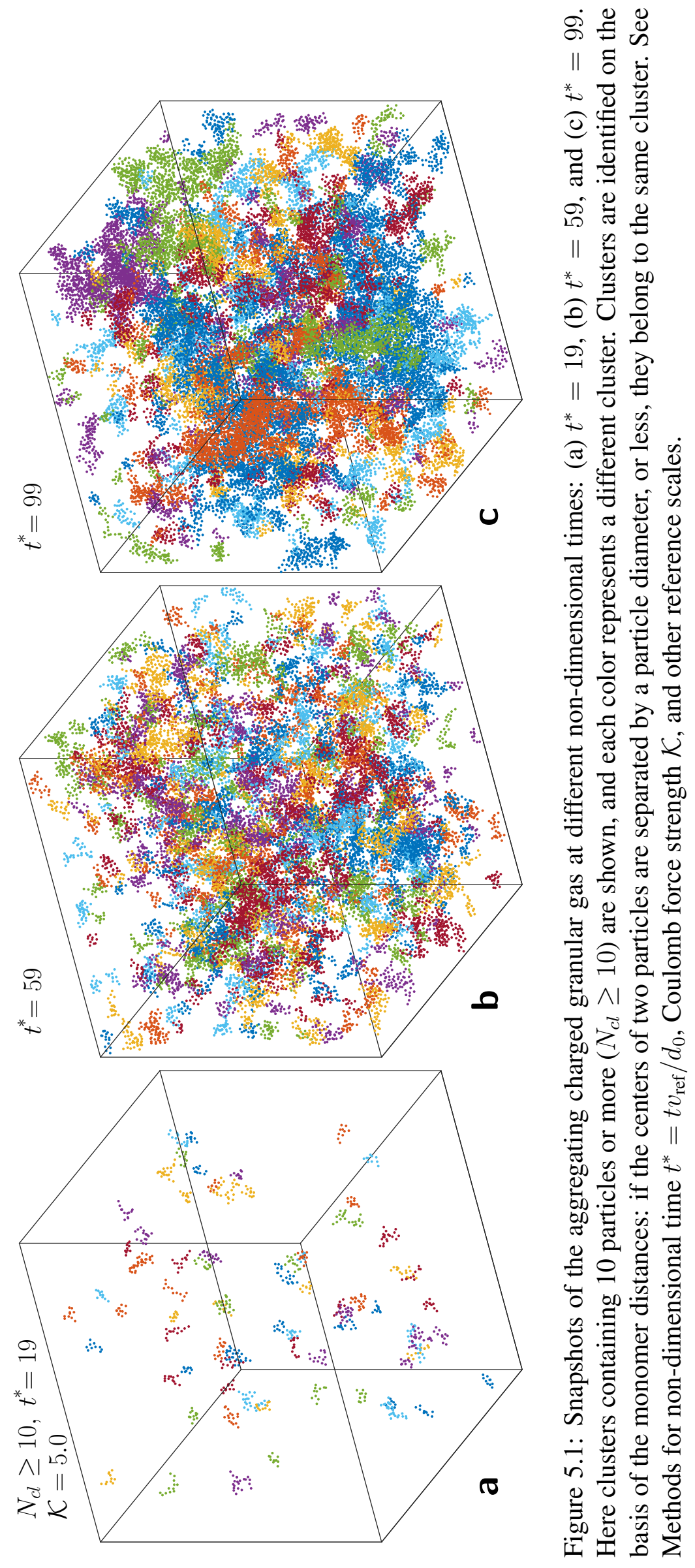


valid at any time instant $t$. Here we define $I_{\text {coll }}$ as the modified collision integral which includes dissipation as well as charge exchange during particle collisions. We will now elucidate how the charge exchange mechanism and particle size growth modify the collisions.

Let us consider contact collisions of particles $i$ and $j$ with pre-collision velocity-charge values $\left(\mathbf{v}_{i}, q_{i}\right)$ and $\left(\mathbf{v}_{j}, q_{j}\right)$, respectively. In the ensemble picture, particle collisions will change $f(t ; \mathbf{v}, q)$ in the infinitesimal phase-space volumes $d \mathbf{v}_{i} d q_{i}$ and $d \mathbf{v}_{j} d q_{j}$, centered around $\left(\mathbf{v}_{i}, q_{i}\right)$ and $\left(\mathbf{v}_{j}, q_{j}\right)$, respectively. The number of direct collisions $N_{c}^{-}$per unit spatial volume which lead to loss of particles from the intervals $d \mathbf{v}_{i} d q_{i}$ and $d \mathbf{v}_{j} d q_{j}$ in time $\Delta t$ are

$$
N_{c}^{-}=f_{i} d \mathbf{v}_{i} d q_{i} f_{j} d \mathbf{v}_{j} d q_{j}\left|\mathbf{v}_{i j} \cdot \mathbf{n}\right| \Theta\left(-\mathbf{v}_{i j} \cdot \mathbf{n}\right) \Theta_{q} d \sigma \Delta t
$$

where $\mathbf{v}_{i j} \equiv \mathbf{v}_{j}-\mathbf{v}_{i}, \mathbf{n}$ is the unit vector at collision pointing from the center of particle $i$ towards particle $j$, and $d \sigma$ is the differential collision cross-section. The Heaviside step function $\Theta\left(-\mathbf{v}_{i j} \cdot \mathbf{n}\right)$ selects particles coming towards $i$, while we use $\Theta_{q} \equiv \Theta\left(\frac{1}{2} m v_{i j}^{2}-\frac{k_{e} q_{i} q_{j}}{d}\right)$ to ensure that a contact with an approaching particle takes place only when the Coulomb energy barrier can be overcome, where $k_{e}=1 /\left(4 \pi \varepsilon_{0}\right)$, $\varepsilon_{0}=8.854 \times 10^{-12} \mathrm{~F} \mathrm{~m}^{-1}$ is the vacuum permittivity, and $d$ is the particle diameter at time $t$. If the interaction is repulsive, $k_{e} q_{i} q_{j} / d$ is positive, and $\Theta_{q}=1$ only if $\frac{1}{2} m v_{i j}^{2}>k_{e} q_{i} q_{j} / d$. In case of attractive interaction, $k_{e} q_{i} q_{j} / d$ is negative and thus $\Theta_{q}=1$ always. Essentially, $\Theta_{q}$ filters repulsive interactions which do not lead to a physical contact between particles.

Consider now particles with initial velocity-charge values $\left(\mathbf{v}_{i}^{\prime \prime}, q_{i}^{\prime \prime}\right)$ and $\left(\mathbf{v}_{j}^{\prime \prime}, q_{j}^{\prime \prime}\right)$ in the intervals $d \mathbf{v}_{i}^{\prime \prime} d q_{i}^{\prime \prime}, d \mathbf{v}_{j}^{\prime \prime} d q_{j}^{\prime \prime}$. The number of particles $N_{c}^{+}$per unit volume which, postcollision, enter the interval $d \mathbf{v}_{i} d q_{i}$ and $d \mathbf{v}_{j} d q_{j}$ in time $\Delta t$ is

$$
N_{c}^{+}=f_{i}^{\prime \prime} d \mathbf{v}_{i}^{\prime \prime} d q_{i}^{\prime \prime} f_{j}^{\prime \prime} d \mathbf{v}_{j}^{\prime \prime} d q_{j}^{\prime \prime}\left|\mathbf{v}_{i j}^{\prime \prime} \cdot \mathbf{n}\right| \Theta\left(-\mathbf{v}_{i j}^{\prime \prime} \cdot \mathbf{n}\right) \Theta_{q}^{\prime \prime} d \sigma^{\prime \prime} \Delta t
$$

The net change $\Delta N_{c} \equiv N_{c}^{+}-N_{c}^{-}$of number of particles in time $\Delta t$ per unit volume, then reads (see Methods)

$$
\Delta N_{c}=\left(\frac{1}{\epsilon\left(v_{i j}\right)} J_{i j}^{v_{i j}} J_{i j}^{q} f_{i}^{\prime \prime} f_{j}^{\prime \prime}-f_{i} f_{j}\right)\left|\mathbf{v}_{i j} \cdot \mathbf{n}\right| \Theta\left(-\mathbf{v}_{i j} \cdot \mathbf{n}\right) d \mathbf{v}_{j} d q_{j} d \sigma \Theta_{q} \Delta t
$$


where $J_{i j}^{v_{i j}}$ and $J_{i j}^{q}$ are the determinants of the Jacobians of the transformations for $d \mathbf{v}_{i}^{\prime \prime} d \mathbf{v}_{j}^{\prime \prime} \rightarrow d \mathbf{v}_{i} d \mathbf{v}_{j}$ and $d q_{i}^{\prime \prime} d q_{j}^{\prime \prime} \rightarrow d q_{i} d q_{j}$, respectively, which lump together the microscopic details of the collision process, namely dissipation and charge exchange in the present study.

Integrating over all incoming particle velocities and charges from all directions, dividing by $\Delta t$ and taking the limit $\Delta t \rightarrow 0$, we obtain the formal expression for the modified collision integral

$$
I_{\text {coll }}=\int\left(\frac{1}{\epsilon\left(v_{i j}\right)} J_{i j}^{v_{i j}} J_{i j}^{q} f_{i}^{\prime \prime} f_{j}^{\prime \prime}-f_{i} f_{j}\right)\left|\mathbf{v}_{i j} \cdot \mathbf{n}\right| \Theta\left(-\mathbf{v}_{i j} \cdot \mathbf{n}\right) d \mathbf{v}_{j} d q_{j} d \sigma \Theta_{q}
$$

Here we assume that the differential collision cross section and the contact condition specified by $\Theta_{q}$ retain their form for direct and inverse collisions. The particle encounters which do not lead to a physical contact have been excluded using $\Theta_{q}$. While taking moments of $I_{\text {coll }}$, a fraction of those contact collisions that lead to aggregation is accounted for by taking the limit $\epsilon=0$ for certain conditions on the relative velocity $v_{i j}$, and by considering the charge transferred to particle $i$ equal to the charge on particle $j$ (see Methods). In $I_{\text {coll }}$, distant encounters, which do not lead to a contact between particles (glancing collisions) are neglected and the charge exchange and dissipation is considered only during the contact. The long-range effect is incorporated via the collision cross-section.

After setting up the collision integral, we derive the macroscopic changes of number density $n$, granular temperature $T$, and the charge variance $\left\langle\delta q^{2}\right\rangle$ by taking the moments of the Boltzmann equation (see Methods). The particles are initially neutral and the charge on them is altered either by collisions or during aggregation. However, due to charge conservation during collisions and aggregation, the system remains globally neutral and the mean charge variation $\langle\delta q\rangle$ is zero. The next choice is thus $\left\langle\delta q^{2}\right\rangle$. In order to obtain closed-form equations, and for analytical tractability, we assume quasi-monodispersity and homogeneity of the aggregating granular gas at any given time, as illustrated in Fig. 5.2. This means that during aggregation the mass of the clusters is assumed to grow homogeneously, while their numbers decrease in a given volume.

We assume that the charge and velocity distributions are uncorrelated, and their properly scaled form remains Gaussian (see Methods). After integration, we find the governing 


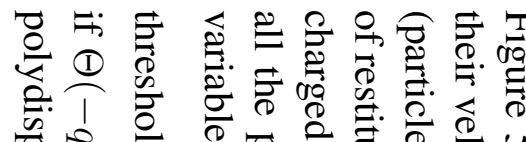

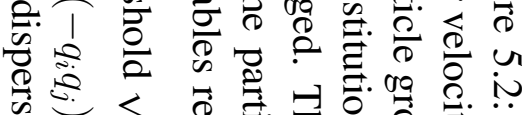

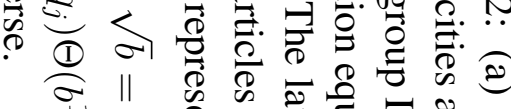

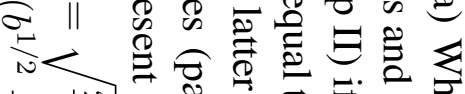

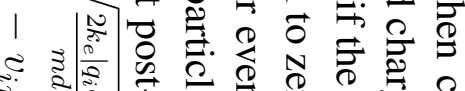

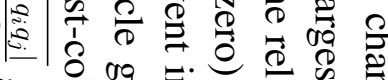

ᄂ

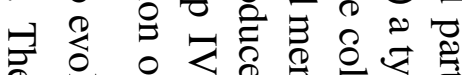

के

ซै ¿

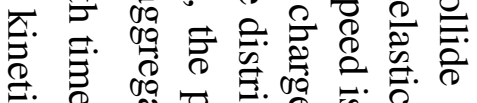
ने के एव

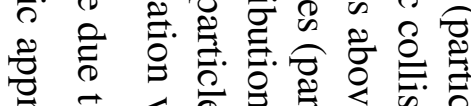

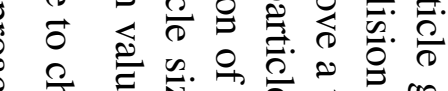

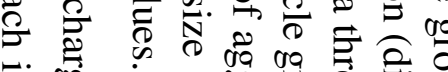
ज.

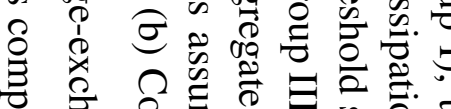

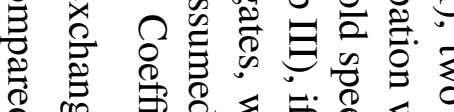

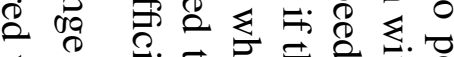

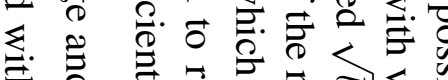

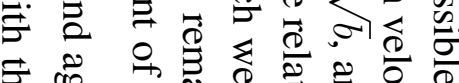

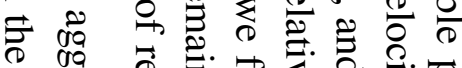

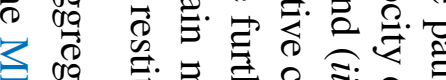

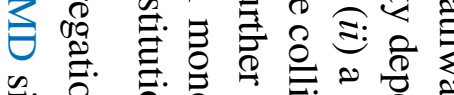

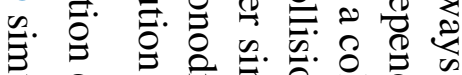

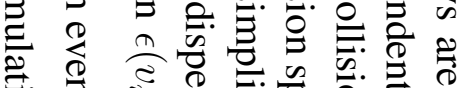

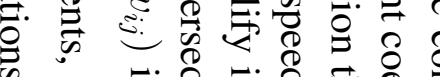
क $\because \stackrel{\circ}{2} \Xi \stackrel{\varnothing}{\circ}$ ङ

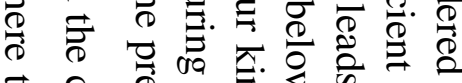
ह $\delta$ क

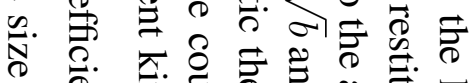

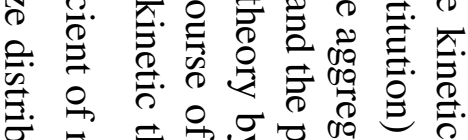

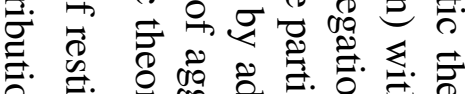

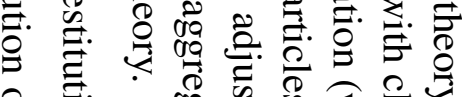

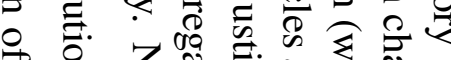

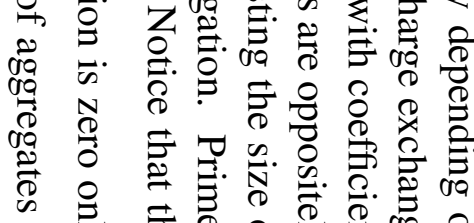

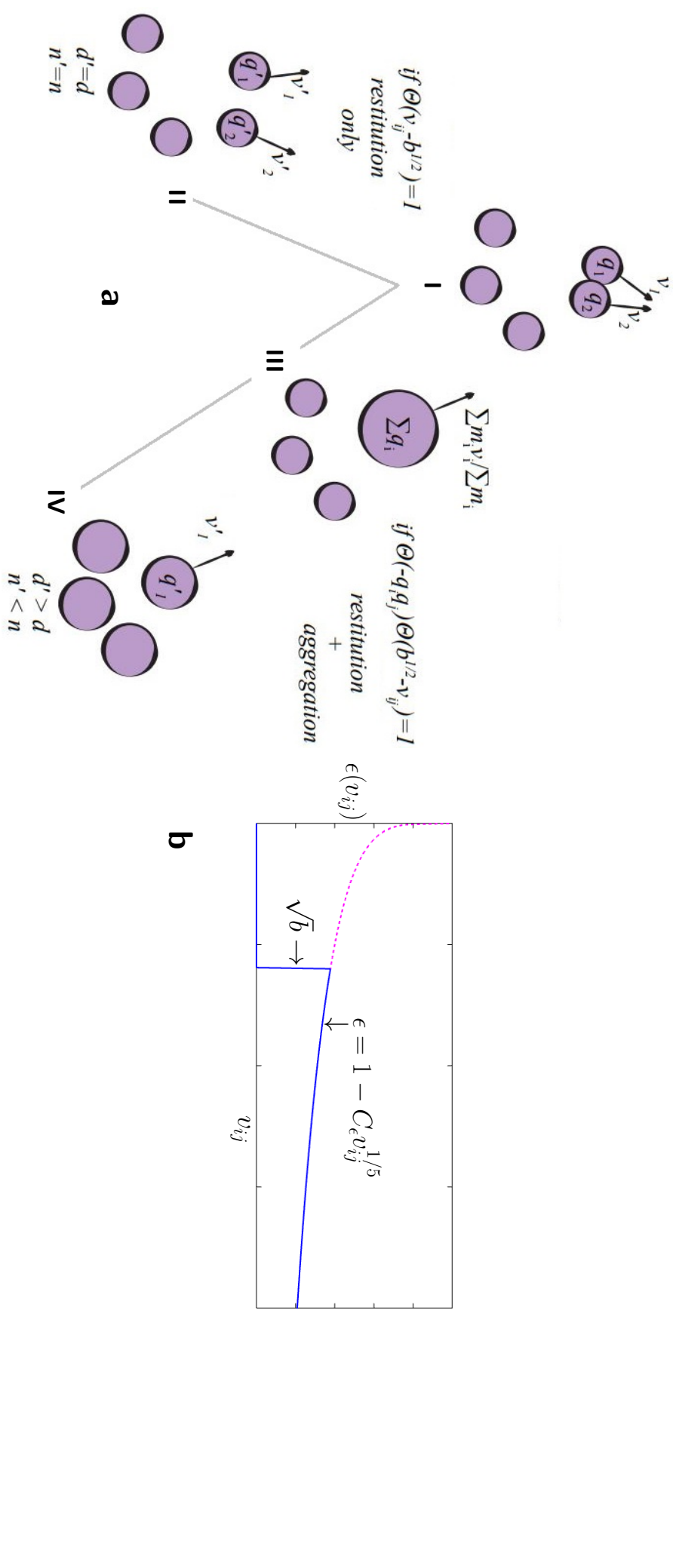


equations

$$
\begin{gathered}
\frac{\partial n}{\partial t}=-n^{2} T^{\frac{1}{2}} g_{1}\left(\mathcal{B}, C_{\text {agg }}^{n}\right), \\
\frac{3}{2} \frac{\partial T}{\partial t}=-n^{2} T^{\frac{8}{5}} g_{2}\left(\mathcal{B}, C_{r e s}^{T}\right)+n^{2} T^{\frac{3}{2}} g_{3}\left(\mathcal{B}, C_{a g g}^{T}\right), \\
\frac{\partial\left\langle\delta q^{2}\right\rangle}{\partial t}=n^{2} T^{\left(\eta+\frac{1}{2}\right)} g_{4}\left(\mathcal{B}, C_{r e s}^{q}\right)-n^{2}\left\langle\delta q^{2}\right\rangle T^{\frac{1}{2}} g_{5}\left(\mathcal{B}, C_{a g g}^{q}\right),
\end{gathered}
$$

which are coupled via a time-dependent dimensionless ratio

$$
\mathcal{B}(t) \equiv k_{e} \frac{\left\langle\delta q^{2}\right\rangle(t)}{T(t) d(t)}
$$

between charge variance, granular temperature, and aggregate size. The terms $g_{k}$ are timedependent functions of $\mathcal{B}$ and material constants $C_{\text {res }}, C_{a g g}$ (Methods Table I). We term the ratio $\mathcal{B}$ as Bjerrum number. In Eq. (5.9), $d$ represents the size of a particle, also evolving with time during aggregation [Fig. 5.2]. Notice that as $f$ is considered independent of $d$ during aggregation, an explicit equation for $d$ is required. For this we consider the total mass $M$, system volume $V$, and particle material density $\rho_{\mathrm{m}}$ to be constant, which fixes the relation between particle size $d$ and particle number density $n$, according to

$$
d(t)=\left[\frac{6 M}{\pi n(t) V \rho_{\mathrm{m}}}\right]^{\frac{1}{D_{f}}},
$$

and closes the equation set (5.6)-(5.8). Here $D_{f}$ is the fractal dimension. In the solution of the above analytical equations, we have assumed $D_{f}=3$ (spherical aggregates). Below, we will compute values of $D_{f}$ using the MD simulations. The above set of equations is consistent with modified Haff's law for a velocity dependent coefficient of restitution (Schwager \& Pöschel, 1998; Brilliantov \& Pöschel, 2000b; Ramírez et al. , 1999) in the absence of collisional charging. In this limit $\left\langle\delta q^{2}\right\rangle=0, \mathcal{B}=0$, and we obtain $\frac{\partial n}{\partial t}=0$, $\frac{\partial\left\langle\delta q^{2}\right\rangle}{\partial t}=0$ and $\frac{3}{2} \frac{\partial T}{\partial t}=-T^{8 / 5}\left[\frac{\pi C_{r e s}^{T}}{2}\right]$, whose solution is the modified Haff's law. 


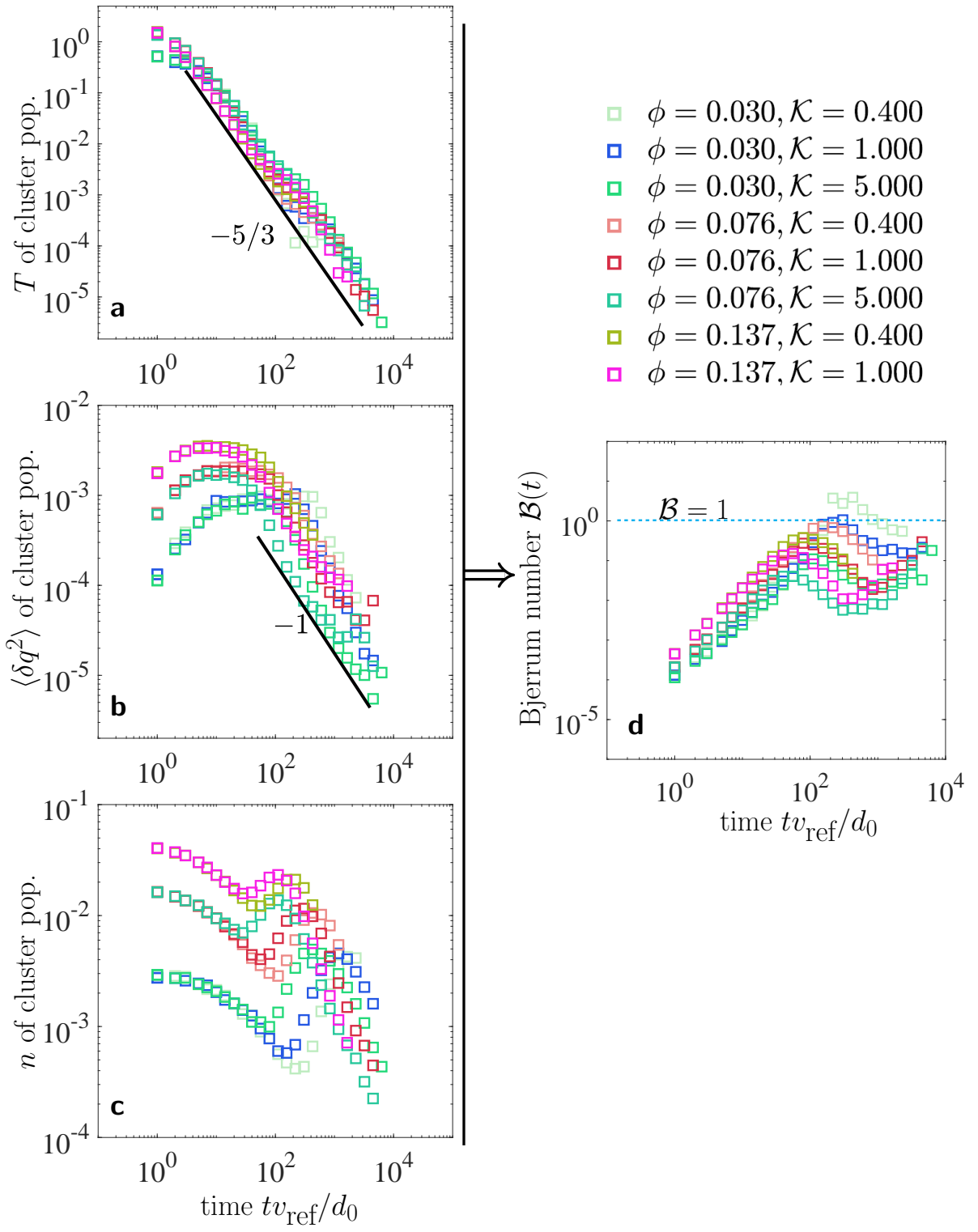

Figure 5.3: Evolution of temperature (a) $T$ of cluster population, (b) charge variance $\left\langle\delta q^{2}\right\rangle$ of cluster population, and (c) number density $n$ of cluster population, for different monomer filling fractions $\phi$ and charge strength $\mathcal{K}$. (d) The granular temperature, charge variance and average size of the cluster population during aggregation evolve in such a manner that their non-dimensional combination $\mathcal{B}(t)=k_{e}\left\langle\delta q^{2}\right\rangle /(T d) \leq 1$ (see also Fig. 5.4). Both temperature and charge variance of cluster population decay as power laws. The number density evolution, however, is highly dynamic and exhibits a nonmonotonic behavior due to emergence of mesoscopic flow (see Methods for $\phi, \mathcal{K}, v_{\text {ref }}, d_{0}$ and mesoscopic flow). 


\subsection{Results}

The time evolution of $T,\left\langle\delta q^{2}\right\rangle$ and $n$ of aggregate population from MD simulations is shown in Fig. 5.3. The aggregate temperature from simulations is extracted as $\frac{3}{2} T=$ $\frac{1}{N_{\text {agg }}} \sum_{i} \frac{1}{2} m_{i}\left[\left(\mathbf{v}_{i}-\mathbf{V}\right)^{2}\right]$. Notice that $\mathbf{v}_{i}$ and $m_{i}$ are center of mass velocity, and mass of the $i^{\text {th }}$ aggregate respectively, and should not be confused with monomer velocities and masses. $\mathbf{V}$ is the local advective velocity in the neighborhood of $i^{\text {th }}$ aggregate. Similarly, $\left\langle\delta q^{2}\right\rangle=\frac{1}{N_{\text {agg }}} \sum_{i}\left(q_{i}-\langle q\rangle\right)^{2}$, and $n=N_{\text {agg }} / V$, where $N_{\text {agg }}$ is the total number of aggregates and $V$ is the system volume. In the MD simulations, oppositely charged monomers hold together into mechanical contact when the elastic repulsion between them balances the Coulomb attraction. This is the scenario at low granular temperature when the dissipative force term, and the inertial term, in the equation of motion [Eq. (5.57)] is negligible compared to the elastic and Coulomb terms. We identify the aggregates with the following condition: if the distance between the centers of two monomers $r_{i j}$ is less than or equal to the monomer diameter $d_{0}$, they belong to the same aggregate. Once all the aggregates in the system are identified using the above definition, the aggregate velocity and mass as a whole is computed. If $v_{i}$ are the velocities of monomers in $k^{\text {th }}$ aggregate, the aggregate velocity $v_{k}$ is computed by $v_{k}=\sum_{i} m_{i} v_{i} / \sum_{i} m_{i}$, which is the center of mass velocity of the aggregate. The mass of the aggregate is simply $m_{k}=\sum_{i} m_{i}$. Similarly, the net charge on $k^{\text {th }}$ aggregate is $q_{k}=\sum_{i} q_{i}$.

Initially $\left(t v_{\text {ref }} / l_{\text {ref }}<10^{2}\right)$, the relative collision velocities $v_{i j}$ remain larger than the time varying threshold $\sqrt{b} \equiv \sqrt{\frac{k_{e}\left|q_{i} q_{j}\right|}{2 T d}}$ (see Methods for the treatment of threshold $b$ ). In this time regime, the collisions are primarily restitutive, leading to either Coulomb scattering without collision, or charge exchange and dissipation without considerable aggregation. The dissipation reduces $T$ [Fig. 5.3(a)] while the charge exchange increases $\left\langle\delta q^{2}\right\rangle$ [Fig. 5.3(b)]. In this time regime the number density $n$, and thus size $d$ of the aggregates, is altered only moderately due to those low-velocity attractive monomer encounters which lead to aggregation [Fig. 5.3(c)].

As a result of our kinetic formulation, the dynamics of $n, T$ and $\left\langle\delta q^{2}\right\rangle$ can be collected into evolution of $\mathcal{B}$, shown in Fig. 5.3(d). The Bjerrum number $\mathcal{B}$ initially increases, which indicates that temperature decreases at a faster rate than the rate of increase of charge variance and the aggregate size. As the relative velocities $v_{i j}$ approach the thresh- 
old $\sqrt{b}, \mathcal{B} \rightarrow 1$. Near this time, the dynamics cross over to aggregative collapse. The individual particles, or monomers, cluster in such a way that the charge variance of the cluster population now begins to reduce. The temperature of the aggregate population keeps decreasing at the same rate with a slight dip near the aggregative collapse. The number density starts to evolve non-monotonically. We explore the non-monotonicity in the next section and in the Methods. These results are robust under variation of the initial monomer filling fraction $\phi$ and the charge strength $\mathcal{K}$ [Fig. 5.3].

After the initial time regime and the aggregative collapse, the crossover in the dynamics is depicted in Fig. 5.4, where the evolution of the combination $\mathcal{B}$, and its comparison with the solution of Eq. (5.6)-(5.8) is highlighted. We solve the full kinetic equations Eq. (5.6)-(5.8) including the aggregation kinetics [Fig. 5.4 (solid line)]. We also solve Eq. (5.6)-(5.8) for a system with only dissipative collisions and without aggregation; hence, the cluster size $d$ remains unchanged. These results are shown in Fig. 5.4 (dashed line). In this limit of only restitutive kinetics, $\mathcal{B}$ increases continuously above the limiting value 1 . The purely restitutive kinetic theory thus fails to predict the MD results. When aggregation is explicitly treated (solid line), the theory predicts an upper limit during the growth. The theory shows that once aggregation sets in, the aggregating granular gas obeys the constraint

$$
\mathcal{B}(t) \leq 1
$$

The upper physical limit predicted in the theory, $\mathcal{B}(t)<1$, is endorsed by the granular MD simulations under moderate variation of $\phi$ and $\mathcal{K}$. It is notable that at later times, the limit $\mathcal{B} \leq 1$ allows the right-hand sides of the equations for number density, temperature and charge variance [Eq. (5.6)-(5.8)] to remain real-valued during the aggregation process. This mathematical indication confirms the effectiveness of the quasi-monodisperse picture [Fig. 5.2] considered in the present study.

The initiation of aggregation brings about a power law decay in the charge variance [Fig. 5.3(b)]. It is notable that a different charge exchange model might provide a different charge buildup rate during the purely dissipative (restitutive) phase. However, the decay of charge variance during the aggregative phase is not expected to be influenced by charge exchange mechanism. The reason is that aggregation sets in at relatively low 
temperature where the thermal motion of monomers, if any, inside the clusters is significantly decreased, and thus collisional charge-exchange is expected to be negligible. If two oppositely charged particles $i$ and $j$ collide with a speed which is below the aggregation threshold speed, it is considered that they form a single aggregate with net charge $q_{i}+q_{j}$ on it [Fig. 5.2]. Thus, the charge variance of the cluster population is reduced by the aggregation process, rather than by the collisional charge exchange.

Our key theoretical finding is that after the aggregative collapse, the decay of the charge variance of aggregate population and the growth of the size of the aggregates is balanced by the decay of temperature during the aggregation, resulting in the stationary value of $\mathcal{B}(t)$. The constraint $\mathcal{B} \leq 1$ is robust in the theory, while the granular MD simulations suggest $\mathcal{B}(t)<1$ and confirm the upper limit of $\mathcal{B}(t)$. It is also intriguing that the temperature of the cluster population still closely follows the modified Haff's law for different $\phi$ and $\mathcal{K}$, despite complex heterogeneous aggregation-fragmentation events and the long-range electrostatic interactions.

The number density's temporal evolution obtained from the MD simulations reveals a more intricate non-monotonic dynamics. It initially begins to decrease during small aggregate formations due to low-velocity attractive monomer encounters. In an intermediate time regime, the emergence of mesoscopic particle fluxes triggers fragmentation events and the aggregate numbers increase. We quantify the emergence of mesoscopic flow using the Mach number (see Methods and Media therein). After this intermediate time regime, the aggregation again takes over and the number density of clusters starts to decrease. The non-monotonic evolution of $n$ causes a dip in $\mathcal{B}(t)$ after the aggregative collapse $\left(t v_{\text {ref }} / l_{\text {ref }}>10^{2}\right)$ [Fig. 5.3 and 5.4]. For temperature values in this regime, the charge transfer events between monomers are statistically ineffective, and the evolution of $\left\langle\delta q^{2}\right\rangle$ is primarily dominated by aggregation events. We find that a maximum of charge fluctuations $\left\langle\delta q^{2}\right\rangle$ occurs near this crossover.

The size difference between aggregates in the MD simulations further adds to the complexity of $\mathcal{B}$ 's evolution after the aggregative collapse, which is neglected in the homogeneous and quasi-monodisperse aggregation kinetic theory. However, the theory still clearly predicts the growth of $\mathcal{B}$ and selects a unique upper limit after the aggregative collapse. To further explore the mechanisms behind the non-monotonic evolution of $n$, we 


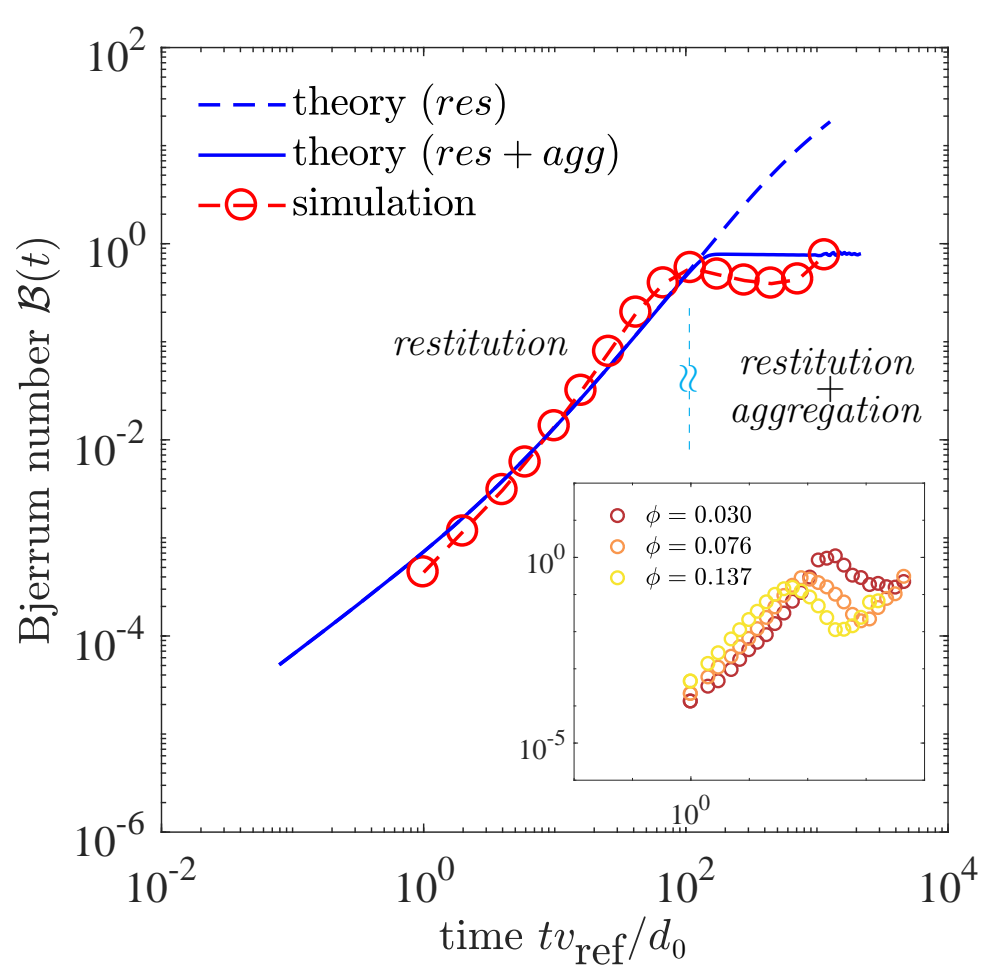

Figure 5.4: The granular temperature, charge variance and average size of the cluster population during aggregation evolve in such a manner that their non-dimensional combination $\mathcal{B}(t)=k_{e}\left\langle\delta q^{2}\right\rangle /(T d) \leq 1$. This is not captured in the kinetic theory if only restitutive (no aggregation) collisions are considered. The granular MD simulations confirm the analytical results. (inset for different monomer filling fraction $\phi$ ).

explore the spatially heterogeneous cluster dynamics and nature of the structures from the MD simulations.

\subsubsection{Inhomogeneous aggregation and fractal growth}

To gain access to the spatial structure formation in the gas, we perform a detailed cluster analysis of the results from granular MD simulation, see Fig. 5.1. The morphology of the aggregates is studied by computing the average fractal dimension $\left\langle D_{f}\right\rangle$ (Mandelbrot, 1977; Jullien, 1987) of cluster population from the scaling relation $m \sim R_{g}^{\left\langle D_{f}\right\rangle}$ between cluster masses $m$, and radii of gyration $R_{g}=\left[\frac{1}{N_{\text {mon }}} \sum_{i}\left(\mathbf{r}_{i}-\mathbf{r}_{\text {mean }}\right)^{2}\right]^{1 / 2}$, where the index $i$ runs over total number of monomers $N_{\text {mon }}$ in a given aggregate. Here $\mathbf{r}_{i}$ is the position vector to the $i^{\text {th }}$ monomer in the given aggregate and $\mathbf{r}_{\text {mean }}$ is the position vector to the center of mass of that aggregate. Once $m$ and $R_{g}$ of all the aggregates in the system are calculated, we compute the $R_{g}$ versus $m$ scatter-plots at different times, for example in Figure 5(a-c). We repeat this for different times and filling fractions, and calculate $\left\langle D_{f}\right\rangle$ as 

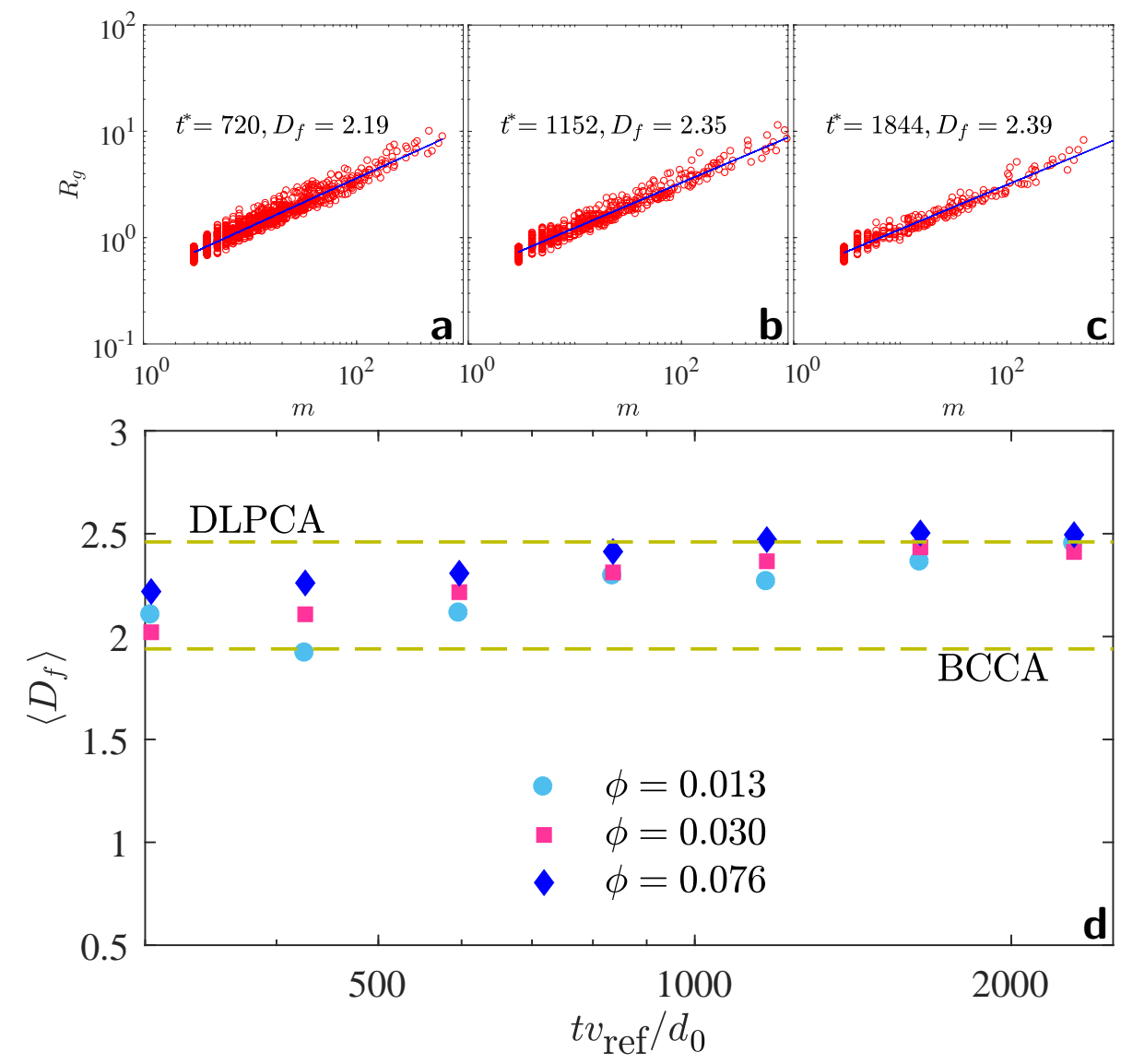

Figure 5.5: (a-c) The scaling between cluster mass $m$ and their radius of gyration $R_{g}$, $m \sim R_{g}^{D_{f}}$ at different times, and (d) the time evolution of $\left\langle D_{f}\right\rangle$, thus obtained, for different filling fractions. The average fractal dimension in the aggregating charged gas varies across reported average values for ballistic cluster-cluster aggregation (BCCA, $\left\langle D_{f}\right\rangle \sim$ 1.94) and diffusion-limited particle-cluster aggregation (DLPCA, $\left\langle D_{f}\right\rangle \sim 2.46$ ) (Blum, 2006; Smirnov, 1990). $t^{*} \equiv t v_{\text {ref }} / d_{0}$. Here the initial transience of the fractal dimension during the charge buildup phase is omitted. 
the slope of the fit to $R_{g}$ versus $m$ scatter-plots. Fig. 5.5(d) shows the time evolution of the exponent $\left\langle D_{f}\right\rangle$ for varying filling fraction. The average fractal dimension lies between the average values reported for the ballistic cluster-cluster aggregation (BCCA, $\left\langle D_{f}\right\rangle \simeq 1.94$ ) and the diffusion-limited particle-cluster aggregation (DLPCA, $\left\langle D_{f}\right\rangle \simeq 2.46$ ) (Blum, 2006; Smirnov, 1990) models. In time, $\left\langle D_{f}\right\rangle$ is dynamic and changes across the two model limits. These results indicate that the aggregate structures retain their fractal nature over time.

The BCCA and DLPCA are popular models for aggregation that have been used for neutral dust agglomeration (e.g hit and stick, ballistic motion, (Blum, 2006)), wet granulate aggregation (sticking due to capillary bridges and ballistic motion, (Ulrich et al. , 2009)), colloidal aggregation (van der Waals and repulsions (Lebovka, 2012)), and hit and stick agglomeration in Brownian particles under frictional drag (Kempf et al. , 1999). The observation that $\left\langle D_{f}\right\rangle$ lies between the reported average values of $\left\langle D_{f}\right\rangle$ for BCCA and DLPCA indicates the presence of mixed characteristics from both of these simplified models. The size distribution in an aggregating charged granular gas (Singh \& Mazza, 2018) tends to resemble a DLPCA-like behavior where the smaller size aggregates are larger in number, in contrast to a BCCA-like model where the size distribution is typically bell-shaped (Blum, 2006). On the other hand, the monomer motion is found to be highly sub-diffusive (Singh \& Mazza, 2018) in agreement with the BCCA model. In addition, the Coulombic interactions will cause considerable deviations from the short-ranged or ballistic propagation typical of the BCCA or DLPCA models. We find that the long-range forces due to a bipolar charge distribution lead to the value of $\left\langle D_{f}\right\rangle$ intermediate between the above two aggregation models, indicated by dashed lines in Fig. 5.5(d).

\subsubsection{Interplay between fractals and mesoscopic flow}

Apart from the long-range effects, the morphology of the aggregates is also altered by additional mechanisms. We discuss two physical processes that are not captured in the analytical theory, but that we investigate via our MD simulations.

First, in our modified Boltzmann kinetic description, the collisions between aggregates at any given time are considered as collisions between two spheres with sizes equal to the average size of the aggregate population. This is a quasi-monodisperse assumption 
typically used in cluster-cluster aggregation models. The quasi-monodispersity, however, neglects the morphology and surface irregularities of the colliding aggregates. In practice, collisions between two aggregates with large size difference are also possible. Additionally, individual monomers might collide with large aggregates and result in fragmentation events. Our kinetic description neglects these specific scenarios. It is important to note that the main reason for the mismatch between kinetic theory and MD results for the density evolution [Fig. 5.7(c)] is our neglect of size differences among aggregates in the kinetic theory.

Secondly, granular gases are characterized by the emergence of a convective flow (Hummel et al. , 2016; Brilliantov \& Pöschel, 2010) which we find (see Methods and Media therein), in the present case, induces the non-monotonicity in the temporal evolution of the number density [Fig. 5.3(c)]. Due to the mesoscopic flow, aggregates which are weakly connected are prone to fragmentation. This results in an intermediate regime where the concentration of aggregates increases instead of decreasing.

Excluding the two above mechanisms explains the slight deviation of our quasimonodisperse Boltzmann theory from the non-monotonic behavior of $\mathcal{B}(t)$ after the crossover to aggregative collapse.

\subsection{Methods}

\subsubsection{Kinetics and modified collision integral}

After obtaining number of direct collisions in Eq. (2) in the main text, let us consider the number of particles $N_{c}^{+}$per unit spatial volume having initial velocity-charge values $\left(\mathbf{v}_{i}^{\prime \prime}, q_{i}^{\prime \prime}\right)$ and $\left(\mathbf{v}_{j}^{\prime \prime}, q_{j}^{\prime \prime}\right)$ in the intervals $d \mathbf{v}_{i}^{\prime \prime} d q_{i}^{\prime \prime}$ and $d \mathbf{v}_{j}^{\prime \prime} d q_{j}^{\prime \prime}$ which, post-collision, enter the in the intervals $d \mathbf{v}_{i} d q_{i}$ and $d \mathbf{v}_{j} d q_{j}$ in time $\Delta t$ are

$$
N_{c}^{+}=f_{i}^{\prime \prime} d \mathbf{v}_{i}^{\prime \prime} d q_{i}^{\prime \prime} f_{j}^{\prime \prime} d \mathbf{v}_{j}^{\prime \prime} d q_{j}^{\prime \prime}\left|\mathbf{v}_{i j}^{\prime \prime} \cdot \mathbf{n}\right| \Theta\left(-\mathbf{v}_{i j}^{\prime \prime} \cdot \mathbf{n}\right) \Theta_{q}^{\prime \prime} d \sigma^{\prime \prime} \Delta t
$$


and thus the net increase of number of particles per unit time and volume is $N_{c}^{+}-N_{c}^{-}$. We can relate the primed velocities to the unprimed via

$$
d \mathbf{v}_{i}^{\prime \prime} d \mathbf{v}_{j}^{\prime \prime}=J_{i j}^{v_{i j}} d \mathbf{v}_{i} d \mathbf{v}_{j}
$$

where $J_{i j}^{v_{i j}}=1+(6 / 5) C_{\epsilon} v_{i j}^{1 / 5}+\ldots$ is the determinant of the Jacobians of the transformation for viscoelastic particles (Brilliantov \& Pöschel, 2010). Here $C_{\epsilon}$ is a material constant. To obtain the transformation $d q_{i}^{\prime \prime} d q_{j}^{\prime \prime} \rightarrow d q_{i} d q_{j}$, we consider the ratio of relative charges after and before the collision

$$
r=\frac{q_{i}-q_{j}}{q_{i}^{\prime \prime}-q_{j}^{\prime \prime}}
$$

and in addition we impose charge conservation during collisions

$$
q_{i}+q_{j}=q_{i}^{\prime \prime}+q_{j}^{\prime \prime}
$$

The above two relations finally provide the transformation

$$
d q_{i}^{\prime \prime} d q_{j}^{\prime \prime}=J_{i j}^{q} d q_{i} d q_{j}
$$

where, for example, $J_{i j}^{q}=\frac{2}{r}$ for a constant $r$. This means that the differential chargespace volume element shrinks or expands by a factor of $r / 2$. In general, for velocity and particle pre-charge dependent charge transfer, the expressions of $r$ and $J_{i j}^{q}$ can be quite complicated as it depends on how the charge exchange takes place during collisions and its dependence on myriad factors (such as size, composition, and crystalline properties). Incorporating the above phase-space volume transformations due to collisions, the net change $\Delta N_{c}$ of number of particles per unit phase-space volume and in time $\Delta t$ reads

$$
\Delta N_{c}=\left(\frac{1}{\epsilon\left(v_{i j}\right)} J_{i j}^{v_{i j}} J_{i j}^{q} f_{i}^{\prime \prime} f_{j}^{\prime \prime}-f_{i} f_{j}\right)\left|\mathbf{v}_{i j} \cdot \mathbf{n}\right| \Theta\left(-\mathbf{v}_{i j} \cdot \mathbf{n}\right) d \mathbf{v}_{j} d q_{j} d \sigma \Theta_{q} \Delta t
$$

where we assume that the differential cross-section and the contact condition specified by $\Theta_{q}$ are the same for direct and inverse collisions. Finally, dividing by $\Delta t$, and integrating over all incoming particle velocities and charges from all directions in the limit $\Delta t \rightarrow 0$, 
we obtain a formal expression for the collision integral

$$
I_{\text {coll }}=\int\left(\frac{1}{\epsilon\left(v_{i j}\right)} J_{i j}^{v_{i j}} J_{i j}^{q} f_{i}^{\prime \prime} f_{j}^{\prime \prime}-f_{i} f_{j}\right)\left|\mathbf{v}_{i j} \cdot \mathbf{n}\right| \Theta\left(-\mathbf{v}_{i j} \cdot \mathbf{n}\right) d \mathbf{v}_{j} d q_{j} d \sigma \Theta_{q} .
$$

At this point the particle encounters which do not lead to a physical contact have been excluded using $\Theta_{q}$, however, collisions that lead to aggregation have not been explicitly accounted. We do so by taking the limit $\epsilon=0$ for certain conditions on the relative velocity $v_{i j}$, and by considering the charge transferred to particle $i$ equal to the charge on particle $j$ [Eq. (5.25)-(5.30) below]. In $I_{\text {coll }}$, distant encounters, which do not lead to a contact between particles (glancing collisions) are neglected and the charge exchange and dissipation is considered only during the contact. The long-range effect is incorporated via collision cross-section.

\subsubsection{Splitting restitution and aggregation}

The time rate of change of the average of a microscopic quantity $\psi\left(\mathbf{v}_{i}, q_{i}\right)$ is obtained by multiplying the Boltzmann equation for $f_{i}$ by $\psi_{i}$ and integrating over $\mathbf{v}_{i}, q_{i}$, i.e.

$$
\frac{\partial\langle\psi\rangle}{\partial t}=\int d \mathbf{v}_{i} d q_{i} \psi_{i} \frac{\partial f_{i}}{\partial t}=\int d \mathbf{v}_{i} d q_{i} \psi_{i} I_{\text {coll }}
$$

It can be shown that

$$
\begin{aligned}
\frac{\partial\langle\psi\rangle}{\partial t} & =\int d \mathbf{v}_{i} d q_{i} \psi_{i} I_{\text {coll }} \\
& =\frac{1}{2} \int d \mathbf{v}_{i} d \mathbf{v}_{j} d q_{i} d q_{j} d \sigma f_{i} f_{j}\left|\mathbf{v}_{i j} \cdot \mathbf{n}\right| \Theta\left(-\mathbf{v}_{i j} \cdot \mathbf{n}\right) \Theta_{q} \Delta\left[\psi_{i}+\psi_{j}\right] \\
& =\int d \mathbf{v}_{i} d \mathbf{v}_{j} d q_{i} d q_{j} d \sigma f_{i} f_{j}\left|\mathbf{v}_{i j} \cdot \mathbf{n}\right| \Theta\left(-\mathbf{v}_{i j} \cdot \mathbf{n}\right) \Theta_{q} \Delta\left[\psi_{i}\right],
\end{aligned}
$$

where $\Delta\left[\psi_{i}+\psi_{j}\right]=\left(\psi_{i}^{\prime}+\psi_{j}^{\prime}-\psi_{i}-\psi_{j}\right)$ and $\Delta\left[\psi_{i}\right]=\left(\psi_{i}^{\prime}-\psi_{i}\right)$ is the change of $\psi$ during the collision between pair $i, j$, and the prime denotes a post collision value. We note that the transformations in Eq. (5.13) and (5.16) are reversed back while integrating $I_{\text {coll }}$ weighted with quantity of interest $\psi$. We consider the number density, the kinetic energy or granular temperature, and the charge variance (the system is globally neutral 
and the mean charge variation $\langle\delta q\rangle$ is zero), respectively

$$
\begin{aligned}
\text { (i) } \psi & =n \\
\text { (ii) } \psi & =\frac{1}{2} m v^{2}, \\
\text { (iii) } \psi & =(\delta q)^{2}=\left(q-q_{0}\right)^{2}=\left(q-\frac{q_{i}+q_{j}}{2}\right)^{2}
\end{aligned}
$$

where $q_{0}=\frac{q_{i}+q_{j}}{2}$ is the mean charge on the colliding pair. At this point we differentiate the restitutive or dissipative collisions from aggregative ones by splitting $\Theta_{q} \Delta\left[\psi_{i}\right]$ as

$$
\begin{aligned}
\Theta_{q} \Delta\left[\psi_{i}\right] & =\Delta^{r e s}\left[\psi_{i}\right] \Theta\left(v_{i j}-\sqrt{b}\right) \\
& +\Delta^{a g g}\left[\psi_{i}\right] \Theta\left(-q_{i} q_{j}\right) \Theta\left(\sqrt{b}-v_{i j}\right)
\end{aligned}
$$

where

$$
\sqrt{b} \equiv \sqrt{\frac{2 k_{e}\left|q_{i} q_{j}\right|}{m d}} .
$$

If $v_{i j}>\sqrt{b}$, the particles collide and separate after the collision irrespective of the sign of $q_{i} q_{j}$ (attractive or repulsive). This leads to dissipation of energy with finite non-zero $\epsilon=\epsilon\left(v_{i j}\right)$, and charge exchange according to a specified rule. The aggregative part is zero in this case. If $v_{i j}<\sqrt{b}$ and $q_{i} q_{j}<0$ (attractive encounters at low velocities), the particles collide and aggregate with $\epsilon=0$, and with charge exchange to particle $i$ equal to $q_{j}$. If $v_{i j}<\sqrt{b}$ and $q_{i} q_{j}>0$ (repulsive encounters at low velocities), no physical contact takes place between the particles which leads to neither dissipation nor aggregation $\left(\Theta_{q} \Delta\left[\psi_{i}\right]=0\right)$. Also represented schematically in Fig. (2) in the main text.

The expressions for $\Delta^{r e s}\left[\psi_{i}\right]$ and $\Delta^{a g g}\left[\psi_{i}\right]$ are obtained as follows. The particle number does not change during a dissipative collision but reduces by one in an aggregative collision, i.e.

$$
\begin{gathered}
\Delta_{n}^{r e s}\left[\psi_{i}+\psi_{j}\right]=0, \\
\Delta_{n}^{a g g}\left[\psi_{i}+\psi_{j}\right]_{n}=-1 .
\end{gathered}
$$


For the granular temperature,

$$
\begin{aligned}
\Delta_{T}^{r e s}\left[\psi_{i}+\psi_{j}\right] & =-\frac{1}{2} m\left(1-\epsilon^{2}\right)\left(\mathbf{v}_{i j} \cdot \mathbf{n}\right)^{2}, \\
\Delta_{T}^{a g g}\left[\psi_{i}+\psi_{j}\right] & =-\frac{1}{2} m\left(\mathbf{v}_{i j} \cdot \mathbf{n}\right)^{2},
\end{aligned}
$$

where we take the limit $\epsilon=0$ for the aggregation. The change in the charge variance is obtained as

$$
\begin{aligned}
\Delta_{q}^{r e s}\left[\psi_{i}\right] & =\left(\delta q_{i}^{2}\right)^{\prime}-\left(\delta q_{i}^{2}\right) \\
& =\left(q_{i}^{\prime}-q_{i}\right)^{2}+2\left(q_{i}^{\prime}-q_{i}\right)\left(q_{i}-q_{0}\right)
\end{aligned}
$$

where $\left(q_{i}^{\prime}-q_{i}\right)$ equals the charge transferred to particle $i$ during its collision with particle $j$, and $q_{0}=\frac{q_{i}+q_{j}}{2}$ is the mean charge on the pair. For the charge transfer, based on seminal experiments (Poppe et al. , 2000b; Poppe \& Schräpler, 2005), we consider

$$
\left(q_{i}^{\prime}-q_{i}\right)=C_{\Delta q}\left|\mathbf{v}_{i j} \cdot \mathbf{n}\right|^{\eta} \frac{q_{i}-q_{0}}{\left|q_{i}-q_{0}\right|}
$$

which is also obtainable if charge transferred is considered proportional to the contact area during the course of collisions. In the present simulations, the value of the exponent $\eta=2 \times 0.8$ after the experiments (Poppe et al. , 2000b; Poppe \& Schräpler, 2005), where a power law dependence was found of the charge exchange on the relative collision energy, when silica particles were impacted on a surface. A similar expression can also be derived if the extent of charge transfer is considered dependent on the maximum area of contact during a collision (Kolehmainen et al. , 2017). In the MD simulations, we have verified that while moderate changes of $\eta$ around the experimental value might produce changes in the charge buildup rate of the gas $\left(t^{*} \lesssim 10^{2}\right.$ in Fig. 5.3(b)), however, the dynamics of charge in the aggregation phase are unaffected $\left(t^{*}>10^{2}\right.$ in Fig. 5.3(b)). Once the magnitude of collision velocities $\left|\mathbf{v}_{i j} \cdot \mathbf{n}\right|$ reduces with reducing granular temperature, the value of $\eta$ has a negligible influence on the decay rate of $\left\langle\delta q^{2}\right\rangle$, because it is primarily controlled by aggregation and merging of charges. The power law in Fig. 5.3(b) during the aggregation time regime remains unaffected. 
Using Eq. (5.28) in Eq. (5.27), we get

$$
\Delta_{q}^{r e s}\left[\psi_{i}\right]=C_{\Delta q}^{2}\left|\mathbf{v}_{i j} \cdot \mathbf{n}\right|^{2 \eta}+2 C_{\Delta q}\left|\mathbf{v}_{i j} \cdot \mathbf{n}\right|^{\eta}\left(q_{i}-q_{0}\right)
$$

For aggregation, the charge tranferred to particle $i$ equals the charge on the merging particle $j$, i.e, $q_{i}^{\prime}-q_{i}=q_{j}$, which gives

$$
\begin{aligned}
\Delta_{q}^{a g g}\left[\psi_{i}\right] & =\left(\delta q_{i}^{2}\right)^{\prime}-\left(\delta q_{i}^{2}\right) \\
& =q_{i} q_{j} .
\end{aligned}
$$

Putting Eqs. (5.25), (5.26), (5.29), and (5.30) in (5.23), and then (5.23) in (5.19), the resulting integrals are solved, assuming the statistical independence of charge-velocity distribution function, i.e., $f(\mathbf{v}, q)=f(\mathbf{v}) f(q)$, and assuming that their scaled form remains Gaussian. In addition to the charge exchange, the coefficient of restitution is taken as velocity dependent, i.e

$$
\epsilon=\epsilon\left(\left|\mathbf{v}_{i j} \cdot \mathbf{n}\right|\right)=1-C_{\epsilon}\left|\mathbf{v}_{i j} \cdot \mathbf{n}\right|^{1 / 5}+\ldots
$$

while the long-range effects due to Coulomb interactions are taken into account by the change in collision cross section. After integration we obtain Eq. (5)-(7) in the main text. The functions $g_{k}$ in Eq. (5)-(7) have the forms

$$
\begin{aligned}
g_{1}(\mathcal{B})= & {\left[\frac{C_{\text {agg }}^{n}}{l_{1}^{3} l^{3}}\right]\left[a_{1}^{n} \tan ^{-1} \frac{l_{1}}{\mathcal{B}}+a_{2}^{n}+a_{3}^{n} \tan ^{-1} \frac{\mathcal{B}}{l}+a_{4}^{n} \tan ^{-1} \frac{l_{1}}{\mathcal{B}}+a_{5}^{n}+a_{6}^{n} \tan ^{-1} \frac{l}{\mathcal{B}}\right] } \\
g_{2}(\mathcal{B})= & {\left[\frac{C_{\text {res }}^{T}}{l^{5}}\right]\left[a_{1}^{T}+a_{2}^{T} \tan ^{-1} \frac{\mathcal{B}}{l}\right] } \\
g_{3}(\mathcal{B})= & {\left[\frac{C_{\text {agg }}^{T}}{l^{5}}\right]\left[a_{3}^{T}+a_{4}^{T} \cot ^{-1} \frac{\mathcal{B}}{l}+a_{5}^{T}+a_{6}^{T} \tan ^{-1} \frac{\mathcal{B}}{l}\right] } \\
g_{4}(\mathcal{B})= & {\left[\frac{C_{\text {res }}^{q}}{l^{5}}\right]\left[a_{1}^{q}+a_{2}^{q} \tan ^{-1} \frac{\mathcal{B}}{l}\right] } \\
g_{5}(\mathcal{B})= & {\left[C_{\text {agg }}^{q}\right]\left[\frac{1}{a_{3}^{q}}\left(a_{4}^{q}+a_{5}^{q} \tan ^{-1} \frac{\mathcal{B}}{l_{1}}+a_{6}^{q} \tan ^{-1} \frac{l}{\mathcal{B}}\right)\right] } \\
& \quad\left[C_{a g g}^{q}\right]\left[\frac{1}{a_{7}^{q}}\left(a_{8}^{q} \tan ^{-1} \frac{l_{1}}{\mathcal{B}}+a_{9}^{q}+a_{10}^{q} \tan ^{-1} \frac{l}{\mathcal{B}}\right)\right]
\end{aligned}
$$




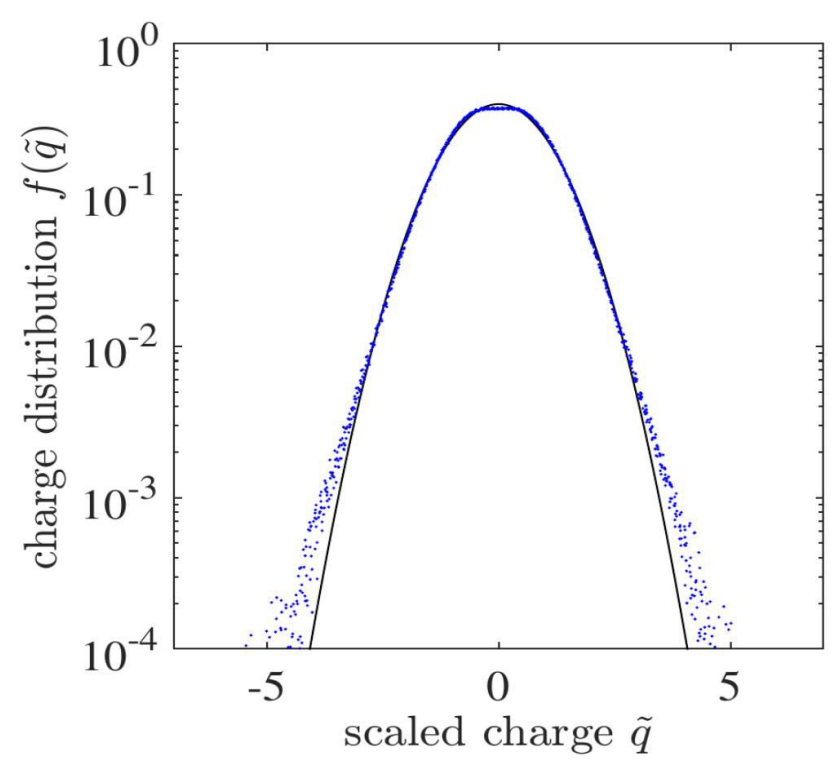

Figure 5.6: The scaled charge distribution $f(\tilde{q})$ of individual particles obtained from typical MD simulation runs (dots) in the aggregated granular gas. The solid line is a Gaussian fit. Here $\tilde{q}=q /\left\langle\delta q^{2}\right\rangle^{1 / 2}$.

where the coefficients $l, l_{1}, a_{k}^{T}, a_{k}^{q}, a_{k}^{n}$ are functions of $\mathcal{B}(t)$ [Table 5.1].

\subsubsection{Derivation of the hydrodynamic equations (5.6)-(5.8)}

To solve the integrals in Eq. (5.19) for different $\Delta\left[\psi_{i}\right]$ from Eq. (5.25)-(5.30), we assume that the normalized velocity as well as charge distribution of the aggregating particle population at any time remain Gaussian, and the two are uncorrelated, i.e

$$
f(\mathbf{v}, q)=f(\mathbf{v}) f(q)=n\left(\frac{m}{2 \pi T}\right)^{\frac{3}{2}} e^{-m v^{2} /(2 T)}\left(\frac{1}{2 \pi\left\langle\delta q^{2}\right\rangle}\right)^{\frac{1}{2}} e^{-q^{2} /\left(2\left\langle\delta q^{2}\right\rangle\right)} .
$$

In Fig. 5.6, we show charge distribution of monomers obtained from typical simulation runs, which is essentially the distribution before initiation of the aggregation. In Eq. (5.38) above, we assume that although granular temperature $T$ and charge variance $\left\langle\delta q^{2}\right\rangle$ do change with time due to restitution and aggregation, the shape of the scaled distribution remains close to Gaussian, and the increase of size/decrease of number of particles due to aggregation process does not alter scaled distribution shape.

The attractive or repulsive long-range effects are emulated through an effective differential cross-section for a binary collision, which changes depending upon the sign and 
magnitude of charges on the particle pair $i, j$ and their relative velocity, according to

$$
d \sigma=\left(\frac{d \sigma}{d \Omega}\right) d \Omega=\frac{d^{2}}{4}\left(1-\frac{2 k_{e} q_{i} q_{j}}{d m\left|\mathbf{v}_{i j} \cdot \mathbf{n}\right|^{2}}\right) d \mathbf{n} \approx \frac{d^{2}}{4}\left(1-\frac{2 k_{e} q_{i} q_{j}}{d m v_{i j}^{2}}\right) d \mathbf{n}
$$

where $\left(\frac{d \sigma}{d \Omega}\right)$ is the differential cross-section per unit solid angle $d \Omega \equiv d \mathbf{n}$. The expression $\frac{d^{2}}{4}\left(1-\frac{2 k_{e} q_{i} q_{j}}{d m v_{i j}^{2}}\right)$ is independent of $\mathbf{n}$, and thus the total cross section is $\sigma=$ $\frac{d^{2}}{4}\left(1-\frac{2 k_{e} q_{i} q_{j}}{d m v_{i j}^{2}}\right) \int_{0}^{\pi} d \phi d \theta \sin \theta=\pi d^{2}\left(1-\frac{2 k_{e} q_{i} q_{j}}{d m v_{i j}^{2}}\right)$. For neutral particles, $q_{i}=q_{j}=0$, and thus $\sigma=\pi d^{2}$. For $q_{i} q_{j}>0$ (repulsive encounters), $\sigma<\pi d^{2}$, while for $q_{i} q_{j}<0$ (attractive encounters), $\sigma>\pi d^{2}$. Thus Eq. (5.39) is a linear adjustment to the neutral cross-section and is reasonable approximation in case of small angle scattering (Landau \& Lifshitz, 2000). The possibility of negative cross-section for repulsive encounters is eliminated by the switch $\Theta\left(v_{i j}-\sqrt{\frac{2 k_{e}\left|q_{i} q_{j}\right|}{m d}}\right)$.

Below we explain the solution procedure for the restitutive, as well as aggregative, part of the equation for $\frac{\partial T}{\partial t}$. Similar procedure can then followed for the equations for $\frac{\partial n}{\partial t}$ and $\frac{\partial\left\langle\delta q^{2}\right\rangle}{\partial t}$.

Plugging Eq. (5.26) into Eq. (5.23) and then the resulting equation to Eq. (5.19), we find

$$
\begin{aligned}
& \frac{3}{2} \frac{\partial T}{\partial t}=\left(\frac{3}{2} \frac{\partial T}{\partial t}\right)_{r e s}+\left(\frac{3}{2} \frac{\partial T}{\partial t}\right)_{a g g} \\
& =\frac{1}{2} \int d \mathbf{v}_{i} d \mathbf{v}_{j} d q_{i} d q_{j} d \sigma f_{i} f_{j}\left|\mathbf{v}_{i j} \cdot \mathbf{n}\right| \Theta\left(-\mathbf{v}_{i j} \cdot \mathbf{n}\right)\left[-\frac{1}{2} m\left(1-\epsilon^{2}\right)\left(\mathbf{v}_{i j} \cdot \mathbf{n}\right)^{2}\right] \Theta\left(v_{i j}-\sqrt{b}\right) \\
& +\frac{1}{2} \int d \mathbf{v}_{i} d \mathbf{v}_{j} d q_{i} d q_{j} d \sigma f_{i} f_{j}\left|\mathbf{v}_{i j} \cdot \mathbf{n}\right| \Theta\left(-\mathbf{v}_{i j} \cdot \mathbf{n}\right)\left[-\frac{1}{2} m\left(\mathbf{v}_{i j} \cdot \mathbf{n}\right)^{2}\right] \Theta\left(-q_{i} q_{j}\right) \Theta\left(\sqrt{b}-v_{i j}\right),
\end{aligned}
$$

which, after using the above Eq. (5.31) and (5.39), and separating the integrals over $\mathbf{n}, \mathbf{v}$ and $q$, reads as

$$
\frac{3}{2} \frac{\partial T}{\partial t}=\frac{1}{2} \int_{q} d q_{i} d q_{j} f\left(q_{i}\right) f\left(q_{j}\right) \times I_{\mathbf{v}}^{r e s}+\frac{1}{2} \int_{q} \Theta\left(-q_{i} q_{j}\right) d q_{i} d q_{j} f\left(q_{i}\right) f\left(q_{j}\right) \times I_{\mathbf{v}}^{a g g}
$$


where

$$
\begin{aligned}
& I_{\mathbf{v}}^{r e s}=\int_{\mathbf{v}} \Theta\left(v_{i j}-\sqrt{b}\right) d \mathbf{v}_{i} d \mathbf{v}_{j} f\left(v_{i}\right) f\left(v_{j}\right) \times \overbrace{\frac{d^{2}}{4}\left(1-\frac{2 k_{e} q_{i} q_{j}}{m d\left|\mathbf{v}_{i j}\right|^{2}}\right)}^{\frac{d \sigma}{d \Omega}} \times I_{\mathbf{n}}^{r e s}, \\
& I_{\mathbf{v}}^{a g g}=\int_{\mathbf{v}} \Theta\left(\sqrt{b}-v_{i j}\right) d \mathbf{v}_{i} d \mathbf{v}_{j} f\left(v_{i}\right) f\left(v_{j}\right) \times \frac{d^{2}}{4}\left(1-\frac{2 k_{e} q_{i} q_{j}}{m d\left|\mathbf{v}_{i j}\right|^{2}}\right) \times I_{\mathbf{n}}^{a g g},
\end{aligned}
$$

and

$$
\begin{aligned}
I_{\mathbf{n}}^{r e s} & =\int_{\mathbf{n}} d \mathbf{n}\left|\mathbf{v}_{i j} \cdot \mathbf{n}\right| \Theta\left(-\mathbf{v}_{i j} \cdot \mathbf{n}\right)\left(-m C_{\epsilon}\left|\mathbf{v}_{i j} \cdot \mathbf{n}\right|^{11 / 5}+\ldots\right), \\
I_{\mathbf{n}}^{a g g} & =\int_{\mathbf{n}} d \mathbf{n}\left|\mathbf{v}_{i j} \cdot \mathbf{n}\right| \Theta\left(-\mathbf{v}_{i j} \cdot \mathbf{n}\right)\left(-\frac{1}{2} m\left|\mathbf{v}_{i j} \cdot \mathbf{n}\right|^{2}\right),
\end{aligned}
$$

where in the aggregative part, we have set $\epsilon=0$, and $\Theta\left(-q_{i} q_{j}\right)$ selects only the attractive encounters against low velocities selected by $\Theta\left(\sqrt{b}-v_{i j}\right)$, the charge-velocity combination which leads to aggregation. Here

$$
\sqrt{b} \equiv \sqrt{\frac{2 k_{e}\left|q_{i} q_{j}\right|}{m d}}
$$

\subsubsection{Solution for the restitutive part $\left(\frac{3}{2} \frac{\partial T}{\partial t}\right)_{\text {res }}$}

The solution for the parts $I_{\mathbf{n}}^{r e s}, I_{\mathbf{v}}^{r e s}$ are as follows.

$$
\begin{aligned}
I_{\mathbf{n}}^{r e s} & =\int_{\mathbf{n}} d \mathbf{n}\left|\mathbf{v}_{i j} \cdot \mathbf{n}\right| \Theta\left(-\mathbf{v}_{i j} \cdot \mathbf{n}\right)\left(-m C_{\epsilon}\left|\mathbf{v}_{i j} \cdot \mathbf{n}\right|^{11 / 5}+\ldots\right) \\
& =\int_{0}^{\pi} \int_{\pi / 2}^{\pi} d \phi d \theta \sin \theta\left(-m C_{\epsilon}\left|\mathbf{v}_{i j}\right|^{16 / 5}|\cos \theta|^{16 / 5}+\ldots\right) \\
& =-2 \pi \frac{5}{21} m C_{\epsilon}\left|\mathbf{v}_{i j}\right|^{16 / 5}+\ldots
\end{aligned}
$$

Using Eq. (5.47) and (5.38) from the above text, $I_{\mathbf{v}}^{\text {res }}$ reads as

$$
\begin{aligned}
I_{\mathbf{v}}^{r e s}=\int_{\mathbf{v}} \Theta\left(v_{i j}-\sqrt{b}\right) d \mathbf{v}_{i} d \mathbf{v}_{j} & {\left[n\left(\frac{m}{2 \pi T}\right)^{3 / 2} e^{-(m / 2 T) v_{i}^{2}}\right]\left[n\left(\frac{m}{2 \pi T}\right)^{3 / 2} e^{-(m / 2 T) v_{j}^{2}}\right] } \\
& \times\left[-2 \pi \frac{5}{21} m C_{\epsilon}\left|\mathbf{v}_{i j}\right|^{16 / 5}\right] \frac{d^{2}}{4}\left(1-\frac{2 k_{e} q_{i} q_{j}}{m d\left|\mathbf{v}_{i j}\right|^{2}}\right)
\end{aligned}
$$


To perform the integration over the relative velocity $\mathbf{v}_{i j}$, the following transformations are made: (i) $\mathbf{w}_{i j}=\frac{2 T}{m}\left(\mathbf{v}_{i}-\mathbf{v}_{j}\right)$, and (ii) $\mathbf{w}_{c}=\frac{2 T}{m}\left(\mathbf{v}_{i}+\mathbf{v}_{j}\right)$, which also results in $d \mathbf{v}_{i} d \mathbf{v}_{j}=-\frac{1}{8}\left(\frac{m}{4 T}\right)^{-3} d \mathbf{w}_{i j} d \mathbf{w}_{c}$. Incorporating these transformations, we obtain

$$
\begin{aligned}
& I_{\mathbf{v}}^{r e s}=\left[-\frac{n^{2} d^{2}}{4} \frac{1}{8}\left(\frac{m}{4 T}\right)^{-3}\left(\frac{m}{2 \pi T}\right)^{3}\left(\frac{4 T}{m}\right)^{16 / 10}\right] \\
& \times \int_{\mathbf{w}_{i j}} d \mathbf{w}_{i j}\left[\int_{\mathbf{w}_{c}} d \mathbf{w}_{c} e^{-w_{c}^{2}}\right] e^{-w_{i j}^{2}}\left[-2 \pi \frac{5}{21} m C_{\epsilon} w_{i j}^{16 / 5}\right]\left(1-\frac{k_{e} q_{i} q_{j}}{2 T d w_{i j}^{2}}\right) \\
& =-2 T^{8 / 5}\left[\frac{2^{1 / 5} n^{2} d^{2}}{\pi^{3} m^{8 / 5}}\right] \underbrace{\left[4 \pi \int_{0}^{\infty} d w_{c} w_{c}^{2} e^{-w_{c}^{2}}\right]}_{I_{w_{c}}} \\
& \times \underbrace{4 \pi \int_{\sqrt{\frac{k_{e}\left|q_{i} q_{j}\right|}{2 T d}}}^{\infty} d w_{i j} w_{i j}^{2} e^{-w_{i j}^{2}}\left[-2 \pi \frac{5}{21} m C_{\epsilon} w_{i j}^{16 / 5}\right]\left(1-\frac{k_{e} q_{i} q_{j}}{2 T d w_{i j}^{2}}\right)}_{I_{w_{i j}}} .
\end{aligned}
$$

Notice the lower limit on relative velocities,

$$
\sqrt{b} \equiv \sqrt{\frac{k_{e}\left|q_{i} q_{j}\right|}{2 T d}}
$$

is also altered due to the transformation $\mathbf{v}_{i j} \rightarrow \mathbf{w}_{i j}$. The integral $I_{w_{c}}$ gives

$$
I_{w_{c}}=\pi^{3 / 2}
$$

while the integral $I_{w_{i j}}$ is solved as

$$
\begin{aligned}
I_{w_{i j}} & =-\frac{40 \pi^{2}}{21} m C_{\epsilon} \int_{\sqrt{\frac{k_{e}\left|q_{i} q_{j}\right|}{2 T d}}}^{\infty} d w_{i j} w_{i j}^{26 / 5} e^{-w_{i j}^{2}}\left(1-\frac{k_{e} q_{i} q_{j}}{2 T d w_{i j}^{2}}\right) \\
& =-\frac{40 \pi^{2}}{21} m C_{\epsilon} \int_{\sqrt{b}}^{\infty} d w_{i j} w_{i j}^{26 / 5} e^{-w_{i j}^{2}}\left(1-\frac{a}{w_{i j}^{2}}\right) \\
& =-\frac{40 \pi^{2}}{21} m C_{\epsilon} \frac{1}{2}\left[\Gamma\left(\frac{31}{10}, b\right)-a \Gamma\left(\frac{21}{10}, b\right)\right] \\
& \approx-\frac{40 \pi^{2}}{21} m C_{\epsilon} \frac{1}{2}[\Gamma(3, b)-a \Gamma(2, b)] \\
& =-\frac{40 \pi^{2}}{21} m C_{\epsilon} \frac{1}{2}\left[2 e^{-b}\left(1+b+b^{2}\right)-a e^{-b}\left(1+b+b^{2}\right)\right],
\end{aligned}
$$


where $a=\frac{k_{e} q_{i} q_{j}}{2 T d}$ and $b=\frac{k_{e}\left|q_{i} q_{j}\right|}{2 T d}$. Putting $I_{w_{c}}, I_{w_{i j}}$ in $I_{\mathbf{v}}^{r e s}$, and finally $I_{\mathbf{v}}^{r e s}$ in the restitutive part of Eq. (5.41), and integrating over $q_{i}, q_{j}$, we obtain

$$
\begin{aligned}
\left(\frac{3}{2} \frac{\partial T}{\partial t}\right)_{r e s} & =\frac{1}{2} \int_{-\infty}^{\infty} \int_{-\infty}^{\infty} d q_{i} d q_{j} \frac{1}{\sqrt{2 \pi\left\langle\delta q^{2}\right\rangle}} e^{-q_{i}^{2} /\left(2\left\langle\delta q^{2}\right\rangle\right)} \frac{1}{\sqrt{2 \pi\left\langle\delta q^{2}\right\rangle}} e^{-q_{j}^{2} /\left(2\left\langle\delta q^{2}\right\rangle\right)} \\
& \times(-2) T^{8 / 5}\left[\frac{2^{1 / 5} n^{2} d^{2}}{\pi^{3} m^{8 / 5}}\right] \pi^{3 / 2} \frac{\left(-40 \pi^{2}\right)}{21} m C_{\epsilon} \\
& \times \frac{1}{2}\left[2 e^{-b}\left(1+b+b^{2}\right)-a e^{-b}\left(1+b+b^{2}\right)\right] \\
& =-T^{8 / 5}\left[\frac{C_{r e s}^{T}}{l^{5}}\right]\left[a_{1}^{T}+a_{2}^{T} \tan ^{-1} \frac{\mathcal{B}}{l}\right]
\end{aligned}
$$

Notice that if $\mathcal{B}=0$, we recover the classical Haff's law for viscoelastic granular gas.

\subsubsection{Solution for the aggregative part $\left(\frac{3}{2} \frac{\partial T}{\partial t}\right)_{\text {agg }}$}

The solution for the parts $I_{\mathbf{n}}^{a g g}, I_{\mathbf{v}}^{a g g}$ are as follows.

$$
\begin{aligned}
I_{\mathbf{n}}^{a g g} & =\int_{\mathbf{n}} d \mathbf{n}\left|\mathbf{v}_{i j} \cdot \mathbf{n}\right| \Theta\left(-\mathbf{v}_{i j} \cdot \mathbf{n}\right)\left(-\frac{1}{2} m\left|\mathbf{v}_{i j} \cdot \mathbf{n}\right|^{2}\right) \\
& =\int_{0}^{\pi} \int_{\pi / 2}^{\pi} d \phi d \theta \sin \theta\left|\mathbf{v}_{i j}\right||\cos \theta|\left(-\frac{1}{2} m\left|\mathbf{v}_{i j}\right|^{2}|\cos \theta|^{2}\right) \\
& =\frac{\pi}{4} m\left|\mathbf{v}_{i j}\right|^{3} .
\end{aligned}
$$

Using Eq. (5.54) and (5.38) from the above text, and again using the variable transformations (i) $\mathbf{w}_{i j}=\frac{2 T}{m}\left(\mathbf{v}_{i}-\mathbf{v}_{j}\right)$, (ii) $\mathbf{w}_{c}=\frac{2 T}{m}\left(\mathbf{v}_{i}+\mathbf{v}_{j}\right)$, (iii) $d \mathbf{v}_{i} d \mathbf{v}_{j}=-\frac{1}{8}\left(\frac{m}{4 T}\right)^{-3} d \mathbf{w}_{i j} d \mathbf{w}_{c}$, the integral $I_{\mathbf{v}}^{a g g}$ in Eq. (5.43) reduces to

$$
I_{\mathbf{v}}^{a g g}=-T^{3}\left[\frac{2 \sqrt{2} \pi^{2} d^{2}}{m^{2}}\right] \underbrace{\int_{0}^{\sqrt{\frac{k_{e}\left|q_{i} q_{j}\right|}{2 T d}}} d w_{i j} w_{i j}^{2} w_{i j}^{3} e^{-w_{i j}^{2}}\left(1-\frac{k_{e} q_{i} q_{j}}{2 T d w_{i j}^{2}}\right)}_{I_{w_{i j}}} .
$$

Here notice that now the relative velocity limits are from $v_{i j}=0$ to $\sqrt{b}$, the condition for aggregation selected by $\Theta\left(\sqrt{b}-v_{i j}\right)$. Finally putting Eq. (5.55) into aggregative part of Eq. (5.41) and integrating over $q_{i}, q_{j}$, we obtain

$$
\left(\frac{3}{2} \frac{\partial T}{\partial t}\right)_{a g g}=n^{2} T^{3 / 2}\left[\frac{C_{a g g}^{T}}{l^{5}}\right]\left[a_{3}^{T}+a_{4}^{T} \cot ^{-1} \frac{\mathcal{B}}{l}+a_{5}^{T}+a_{6}^{T} \tan ^{-1} \frac{\mathcal{B}}{l}\right] .
$$


Notice that after integrating from $v_{i j}=0$ to $\sqrt{b}$, the integration over $q_{i}, q_{j}$ is to be broken into the sum of two parts, one over $q_{i} \in(-\infty, 0], q_{j} \in[0,+\infty)$, plus a second integral over $q_{i} \in[0,+\infty), q_{j} \in(-\infty, 0]$, to satisfy the aggregative condition set by $\Theta\left(-q_{i} q_{j}\right) \Theta\left(\sqrt{b}-v_{i j}\right)$.

A similar procedure is followed for $\left(\frac{\partial\left\langle\delta q^{2}\right\rangle}{\partial t}\right)_{r e s},\left(\frac{\partial\left\langle\delta q^{2}\right\rangle}{\partial t}\right)_{a g g}$ and $\left(\frac{\partial n}{\partial t}\right)_{a g g}$ using corresponding $\Delta\left[\psi_{i}\right]$. Finally, the key constraint to be noted is that the solutions of the integrals in the rate equation for $T$ are real valued for $\mathcal{B} \leq 4$, while in equations for $\left\langle\delta q^{2}\right\rangle$ and $n$, they are real valued for $\mathcal{B} \leq 1$. The MD simulations confirm that these constraints put a physical limit during the aggregation phase.

\subsubsection{Granular MD simulations}

The equation of motion of the form

$$
\begin{aligned}
\frac{d \mathbf{v}_{i}}{d t}= & \sum_{j}\left[\Theta\left(\xi_{i j}\right)\left(\mathcal{E} \xi_{i j}^{\frac{3}{2}}+\mathcal{D} \xi_{i j}^{\frac{1}{2}} \dot{\xi}_{i j}\right) \mathbf{n}_{j i}\right] \\
& +\mathcal{K} \sum_{k} \sum_{\mathbf{b}}{ }^{\prime} \frac{q_{i} q_{k}}{\left|\mathbf{r}_{k i}+\mathbf{b} L\right|^{3}}\left(\mathbf{r}_{k i}+\mathbf{b} L\right),
\end{aligned}
$$

is solved for each particle with a setup of periodic boundary conditions in a cubic box of size $\left(d_{0} L\right)^{3}=70 d_{0} \times 70 d_{0} \times 70 d_{0}$, where $d_{0}$ is the monomer diameter and $L$ is the non-dimensional system length [Table 5.2, 5.3]. Here $\mathbf{b}=\left(b_{x}, b_{y}, b_{z}\right) \in \mathbb{Z}^{3}$ is a vector of integers representing the periodic replicas of the system in each Cartesian direction. The symbol ' indicates that $k \neq i$ if $\mathbf{b}=0$ to avoid Coulomb interaction of particles with themselves. The non-dimensional numbers in the above equation are $\mathcal{E}=\frac{\alpha l_{\mathrm{ref}}^{3 / 2} t_{\mathrm{ref}}}{m_{\mathrm{ref}} \mathrm{ref}_{\mathrm{ref}}}$, $\mathcal{D}=\frac{\beta l_{\text {ref }}^{1 / 2} t_{\text {ref }}}{m_{\text {ref }}}$ and $\mathcal{K}=\frac{k_{e} q_{\text {ref }}^{2} t_{\text {ref }}}{m_{\text {ref }} v_{\text {ref }} l_{\text {ref }}^{2}}$, with $\alpha$ and $\beta$ being viscoelatic material constants. From practical problems, we select the reference length $l_{\text {ref }} \equiv d_{0}$, time reference $t_{\text {ref }}$, velocity reference $v_{\text {ref }}$, and charge reference $q_{\text {ref }}$ such that the elastic force strength $\mathcal{E} \approx 278$, dissipative force strength $\mathcal{E} / 10$, and Coulomb force strength is varied across $\mathcal{K}=0.4-5.0$ [Table 5.2, 5.3]. The effect of dissipation compared to elastic forces is extensively studied for neutral systems (Brilliantov \& Pöschel, 2010). The variation of Coulomb strength compared to dissipation and elastic forces we have repoted in Singh \& Mazza (2018). The above order of magnitudes of $\mathcal{E}, \mathcal{D}$, and $\mathcal{K}$ also helps to attain an early clustering in non-dimensional time units in a finite size $(N \sim 50000)$ neutral granular gas system [see 
Singh \& Mazza (2018) for more details]. Also $\xi_{i j} \equiv d_{0}-r_{i j}, \dot{\xi}_{i j} \equiv \frac{d \xi}{d t}, \mathbf{n}_{j i}$ is the unit vector pointing from center of in-contact neighbor $j$ towards the center of particle $i$, while $\mathbf{r}_{k i}$ is the distance vector pointing from particle $k$ towards the center of particle $i$.

The equation of charge on particle $i$ may be written as

$$
\frac{d q_{i}}{d t}=\sum_{j}\left[\Theta\left(\xi_{i j}\right) I_{j i}\right]
$$

with $I_{j i}$ being the charge-exchange currents from colliding neighbors $j$ during the course of collision. For any contact neighbor $j$, we approximate its integrated value over the time step $\tau$ by Eq. (5.28), i.e.

$$
\left(q_{i}^{\prime}-q_{i}\right)=\int d q_{i}=\int_{0}^{\tau} I_{j i} d t \approx C_{\Delta q}\left|\mathbf{v}_{i j} \cdot \mathbf{n}\right|^{\eta} \frac{q_{i}-q_{0}}{\left|q_{i}-q_{0}\right|}
$$

After charge-exchange, the long-range Coulomb forces for a setup with periodic boundary conditions in Eq. (5.57) is challenging and conditionally convergent as it depends on the order of summation. We employ the Ewald summation that converges rapidly, and has a computational complexity $\mathcal{O}\left(N^{3 / 2}\right)$. The algorithm is highly parallelized and optimized on graphics processing unit (GPU). In our simulations, the total computing time to reach non-dimensional simulation time $\sim 10^{3}$ for a typical simulation with monomers $N \sim 10^{5}$, including the long-range electrostatic forces, is of the order of weeks. See Singh \& Mazza (2018) for more details.

\subsection{Comparison of individual $T,\left\langle\delta q^{2}\right\rangle$, and $n$ profiles, and emergence of convective flow using Mach number}

In Fig. 5.7, we decompose the theoretical comparison of $\mathcal{B}$, presented in the main text, into individual comparisons of $T,\left\langle\delta q^{2}\right\rangle$, and $n$ profiles for a typical simulation run. The difference between the kinetic theory with and without aggregation is also emphasized.

It is noticeable that the granular temperature of the aggregates closely follows Haff's law, and is confirmed by theory, notwithstanding the presence of long-range effects and intricate aggregation and annihilation events. If only the restitutive terms of the hydrodynamic equations are considered (dashed line), the theory predicts that $T$ drops at a slower 


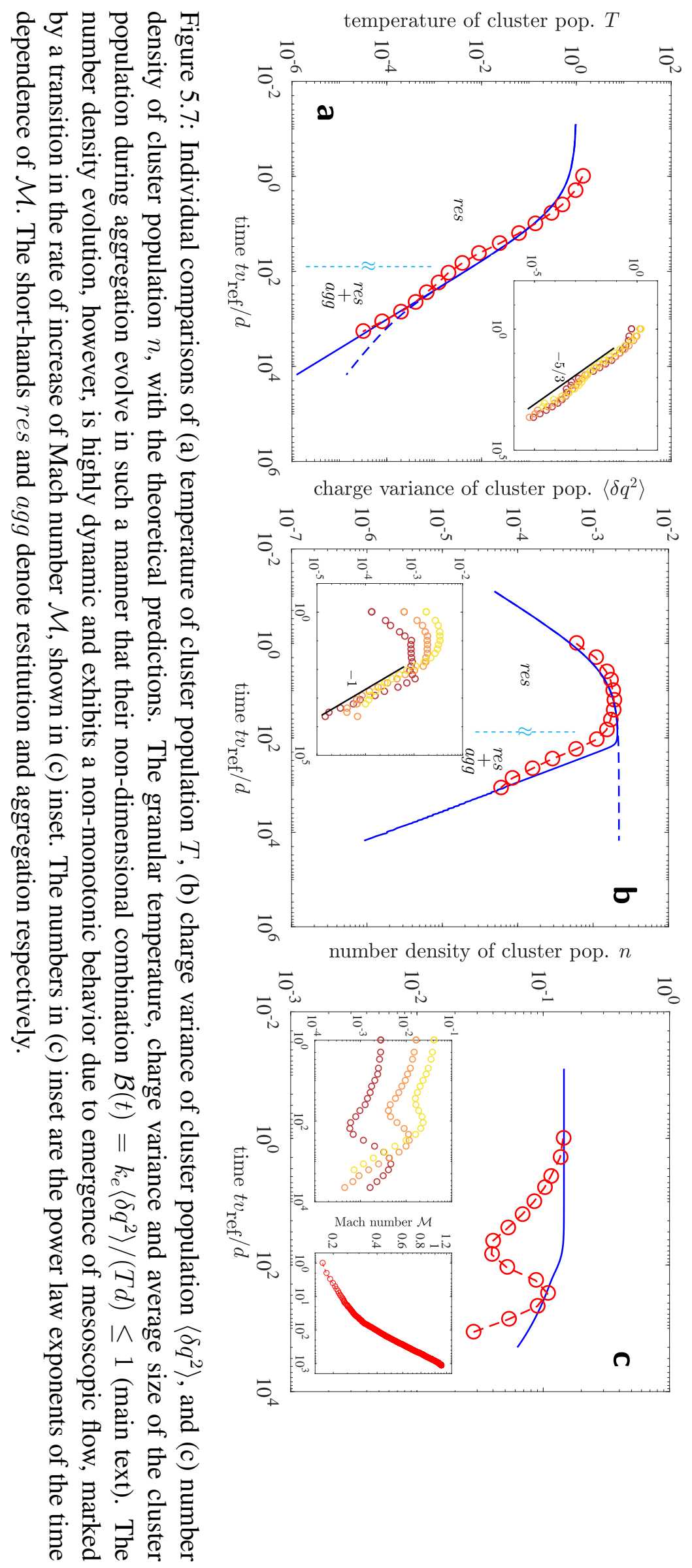


rate at long times. Furthermore, the charge variance in this case saturates. The number density in the absence of aggregation is, of course, invariant. If the aggregation dynamics is augmented, the simulation results are closely predicted by the theory.

In the MD simulations, the decay of $\left\langle\delta q^{2}\right\rangle$ during the aggregation phase closely agrees with the theory, even though we observe that the charge exchange in the simulations leads to asymmetric but non-Gaussian charge distribution among monomers during the initial restitution phase [Fig. 1].

The number density evolution, however, is highly dynamic and exhibits a nonmonotonic behavior due to fragmentation event caused by the emergence of mesoscopic flow. The theory predicts the decay of cluster density only in an average sense [Fig. 5.7(c) inset]. To quantify the emergence of the mesoscopic flow, calculate the Mach number

$$
\mathcal{M}=\frac{\left\langle\mathbf{V}^{2}\right\rangle^{1 / 2}}{v_{\text {th }}}
$$

where $\mathbf{V}$ is the local mesoscopic velocity, and $v_{\text {th }} \sim \sqrt{T}$ is the thermal velocity. To compute this, we divide the system volume into equal sized cubic boxes. The advective velocity in $j^{\text {th }}$ box is computed as $\mathbf{V}_{j}=\sum_{i} m_{i} \mathbf{v}_{i} / \sum_{i} m_{i}$, where index $i$ runs over all the monomers in that box $j$. We take the square of this velocity (we square the velocity of the box before summing it up over all the boxes, otherwise the advective velocities of the boxes might cancel each other even in the presence of a directed motion/advective flow, for example in a lattice of vortices) as $\left\langle\mathbf{V}^{2}\right\rangle=\frac{1}{N_{\text {boxes }}} \sum_{j} \mathbf{V}_{j}^{2}$, take its square root, and normalize it with the thermal velocity $v_{\text {th }} \sim \sqrt{T}$. Thus according to our definition, $\mathcal{M}$ is a global measure of the magnitude of directed/advective motion. The time evolution of $\mathcal{M}$ is shown in Fig. 5.7(c) (inset), which indicates that $\mathcal{M}$ grows at a higher rate when the number density evolution becomes non-monotonic $\left(t v_{\text {ref }} / d_{0} \approx 10^{2}\right)$, indicating an intricate aggregation and fragmentation dynamics, and the generation of mesoscopic flow. 


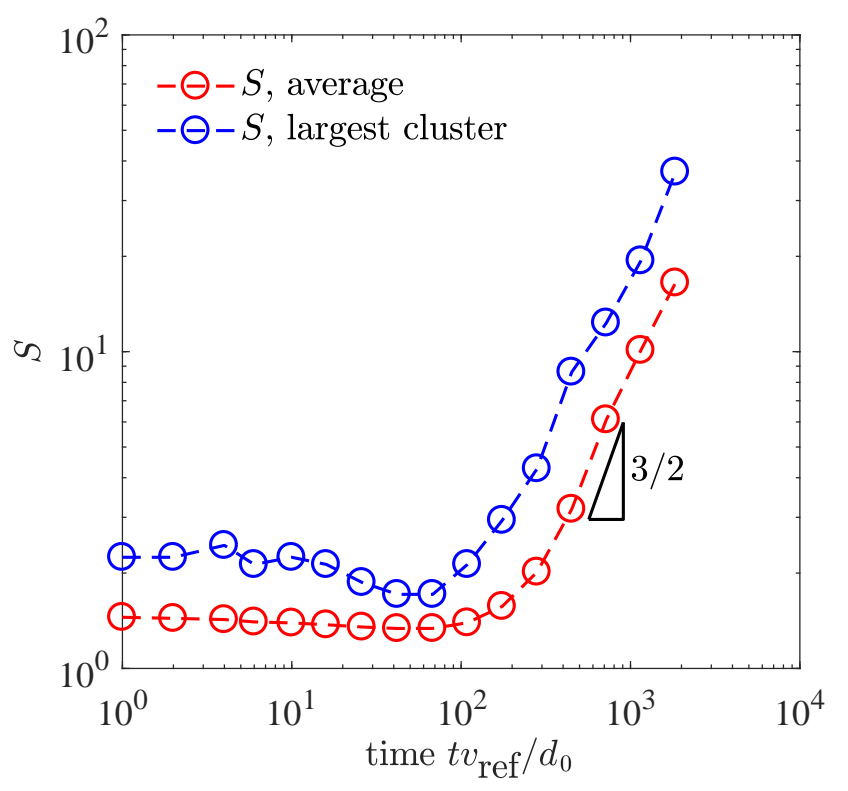

Figure 5.8: Growth of the average cluster size $S$, and the size of the largest cluster with time. Here $S$ is computed from the mass-size relationship $S \sim\langle m\rangle^{1 /\left\langle D_{f}\right\rangle}$. The average mass is defined as $\sum_{k} m_{k}^{2} N_{m}(m) / \sum_{k} m_{k} N_{m}(m)$. Here $\left\langle D_{f}\right\rangle$ is the average fractal dimension, $N_{m}(m)$ is the mass distribution of the aggregates, and the index $k$ runs over all the aggregates. See also Figs. 4.5 and 4.6.

\subsection{Reference scales and laboratory relevance of present}

\section{results}

Typically non-Brownian growth in planetary dust becomes dominant for monomer sizes near or above several $\mu \mathrm{m}$ (Zsom et al. , 2010) and the growth barrier problem (Spahn \& Seiß, 2015) starts to arise near $d \sim 10^{-3} \mathrm{~m}$. The mass of silica particles in this range of sizes is $m \sim 10^{-4}-10^{-6} \mathrm{~kg}$. If the particles are initially agitated with velocities $v \sim 1.0 \mathrm{~m} \mathrm{~s}^{-1}$, the time scale reference to convert our simulation time to laboratory time is $d / v_{\text {ref }} \sim 4.78 \times 10^{-3} \mathrm{~s}$. Thus in our results the growth over $10^{4}$ units of non-dimensional time approximately implies growth over $\sim 10 \mathrm{~s}$. The average size of aggregates in the growth period grows approximately by one order of magnitude (e.g. the growth of the largest cluster is from $\approx 2 \mathrm{~mm}$ to $\approx 7 \mathrm{~cm}$ in $\approx 10 \mathrm{~s} \mathrm{time} \mathrm{for} \mathrm{particles} \mathrm{of} \mathrm{such} \mathrm{size} \mathrm{and}$ mass, and for initial monomer filling fraction of $\phi=0.076$ ) [Fig. 5.8]. 


\subsection{Conclusions and discussion}

We have derived the rate equations for the evolution of the number density $n$, granular temperature $T$, and charge variance $\left\langle\delta q^{2}\right\rangle$ of the cluster population in a collisionally charged, aggregating granular gas [Eqs. (5.6)-(5.8)]. In contrast to well-known Smoluchowski-type equations [Eq. 2.46], we have explicitly coupled $n$ to the decay of $T$ and charge variance. We have compared the results with three-dimensional molecular dynamics simulations [Fig. 5.4 and 5.7] and the outcomes of detailed cluster analysis, and have explored the morphology of the aggregating structures via fractal dimension [Fig 5.5].

Taken together, our results indicate that the aggregation process in a charged granular gas is quite dynamic while respecting some physical constraints. The growth process obeys $\mathcal{B}(t)=k_{e}\left\langle\delta q^{2}\right\rangle /(T d) \leq 1$, while morphologically, the clusters exhibit statistical self-similarity, persistent over time during the growth. The fractal dimension and growth of structures is intermediate between the BCCA and DLPCA models [Fig 5.5]. We also demonstrate that the application of a purely dissipative kinetic treatment is not sufficient to make predictions about global observables such as $T$ and $\left\langle\delta q^{2}\right\rangle$ in an aggregating charged granular gas [Fig 5.4].

Finally, we believe that our kinetic approach can be applied to study aggregation processes in systems such as wet granulates with ion transfer mechanism (Lee et al. , 2018; Zhang et al. , 2015), dissipative cell or active particle collections under long-range hydrodynamic and electrostatic effects (Yan et al. , 2016; Friedl \& Gilmour, 2009), and charged ice-ice collisions (Dash et al. , 2001). 


\begin{tabular}{|c|c|}
\hline Coefficient & Expression \\
\hline $\mathcal{B}$ & $k_{e}\left\langle\delta q^{2}\right\rangle /(T d)$ \\
\hline$l$ & $\sqrt{4-\mathcal{B}^{2}}$ \\
\hline$l_{1}$ & $\sqrt{1-\mathcal{B}^{2}}$ \\
\hline$C_{r e s}^{T}$ & $802^{1 / 5} d^{2} C_{\epsilon} m /\left(21 \sqrt{\pi} m^{8 / 5}\right)$ \\
\hline$C_{a g g}^{T}$ & $d^{2} /(8 \sqrt{\pi m})$ \\
\hline$C_{r e s}^{q}$ & $1.4080 d^{2} C_{\Delta q}^{2}(2+\eta)(4 / m)^{\eta+1 / 2} / \pi$ \\
\hline$C_{a g g}^{q}$ & $4 d^{2} / \sqrt{\pi m}$ \\
\hline$C_{a g g}^{n}$ & $d^{2} /(2 \sqrt{\pi m})$ \\
\hline$a_{1}^{T}$ & $\mathcal{B}\left(8-5 \mathcal{B}^{2}\right) l+\pi\left(16-10 \mathcal{B}^{2}+3 \mathcal{B}^{4}\right)$ \\
\hline$a_{2}^{\frac{1}{T}}$ & $-32+20 \mathcal{B}^{2}-6 \mathcal{B}^{4}$ \\
\hline$a_{3}^{T}$ & $l\left(2 \pi\left(\mathcal{B}^{2}-4\right)^{2}+\mathcal{B}\left(-32+28 \mathcal{B}^{2}+\mathcal{B}^{4}\right)\right)$ \\
\hline$a_{4}^{3}$ & $-4\left(32-20 \mathcal{B}^{2}+9 \mathcal{B}^{4}\right)$ \\
\hline$a_{5}^{T}$ & $l \mathcal{B}\left(-32+28 \mathcal{B}^{2}+\mathcal{B}^{4}\right)$ \\
\hline$a_{6}^{T}$ & $\begin{array}{l}+2 \pi\left(\mathcal{B}^{2}(20-8 l)+\mathcal{B}^{4}(-9+l)+16(-2+l)\right) \\
4\left(32-20 \mathcal{B}^{2}+9 \mathcal{B}^{4}\right)\end{array}$ \\
\hline$a_{1}^{q}$ & $2 \mathcal{B l}\left(\mathcal{B}^{2}-1\right)-\pi\left(4-2 \mathcal{B}^{2}+\mathcal{B}^{4}\right)$ \\
\hline$a_{2}^{q}$ & $2\left(4-2 \mathcal{B}^{2}+\mathcal{B}^{4}\right)$ \\
\hline$a_{3}^{q}$ & $16 l \sqrt{\pi} l_{1}\left(4-5 \mathcal{B}^{2}+\mathcal{B}^{4}\right)^{2}$ \\
\hline$a_{4}^{q}$ & $-\sqrt{\pi} \mathcal{B} l\left(\pi\left(\mathcal{B}^{2}-4\right)\left(1+2 \mathcal{B}^{2}\right)\right.$ \\
\hline & $\left.+2 \mathcal{B} l_{1}\left(-8-54 \mathcal{B}^{2}+33 \mathcal{B}^{4}+2 \mathcal{B}^{6}\right)\right)$ \\
\hline$a_{5}^{q}$ & $2 \sqrt{\pi} \mathcal{B} l^{5}\left(1+2 \mathcal{B}^{2}\right)$ \\
\hline$a_{6}^{q}$ & $16 \sqrt{\pi} \mathcal{B} l_{1}^{5}\left(4+5 \mathcal{B}^{2}\right)$ \\
\hline$a_{7}^{q}$ & $8 l \sqrt{\pi} l_{1}\left(4-5 \mathcal{B}^{2}+\mathcal{B}^{4}\right)^{2}$ \\
\hline$a_{8}^{q}$ & $-\sqrt{\pi} \mathcal{B} l^{5}\left(1+2 \mathcal{B}^{2}\right)$ \\
\hline$a_{9}^{q}$ & $\sqrt{\pi} \mathcal{B} l_{1} \mathcal{B l}\left(8+54 \mathcal{B}^{2}-33 \mathcal{B}^{4}-2 \mathcal{B}^{6}\right)$ \\
\hline$a_{10}^{q}$ & $8 \sqrt{\pi} \mathcal{B} l_{1}\left(\mathcal{B}^{2}-1\right)^{2}\left(4+5 \mathcal{B}^{2}\right)$ \\
\hline$a_{1}^{n}$ & $-\mathcal{B}^{2} l^{3}$ \\
\hline$a_{2}^{n}$ & $l_{1} l \mathcal{B}\left(-4+7 \mathcal{B}^{2}\right)+l_{1} \pi\left(\mathcal{B}^{2}-1\right)\left(8-4 l+\mathcal{B}^{2}(-6+l)\right)$ \\
\hline$a_{3}^{n}$ & $4 l_{1}\left(4-7 \mathcal{B}^{2}+3 \mathcal{B}^{4}\right)$ \\
\hline$a_{4}^{n}$ & $-\mathcal{B}^{2} l^{3}$ \\
\hline$a_{5}^{n}$ & $l_{1} l\left(\mathcal{B}\left(-4+7 \mathcal{B}^{2}\right)+\pi\left(4-5 \mathcal{B}^{2}+\mathcal{B}^{4}\right)\right)$ \\
\hline$a_{6}^{n}$ & $-4 l_{1}\left(4-7 \mathcal{B}^{2}+3 \mathcal{B}^{4}\right)$ \\
\hline
\end{tabular}

Table 5.1: Expressions of the coefficients in Eq. (5)-(7) in the main text. Here $m$ and $d$ are the mass and size of the aggregates. The material constant $C_{\epsilon}$ is from Eq. (5.31) and influence the viscoelastic properties of the particles, while $C_{\Delta q}$ and $\eta$ are from Eq. (5.28) and influence the charge buildup. Other notations are as described in the main text. 


\begin{tabular}{|l|l|l|}
\hline Parameter & Expression & Value \\
\hline$N_{\text {mon }}$ & No. of monomers & $8516,19652,50016,89746$ \\
$L^{3}$ & System size & $70 \times 70 \times 70$ \\
$\phi$ & Monomer filling fraction & $0.013,0.030,0.076,0.137$ \\
$\mathcal{E}$ & $\frac{\alpha l_{\text {ref }}^{3 / 2} t_{\text {ref }}}{m_{\text {ref }} v_{\text {ref }}}$ & 278.0 \\
$\mathcal{D}$ & $\frac{\beta l_{\text {ref }}^{1} / 2 t_{\text {ref }}}{m_{\text {ref }}}$ & 27.8 \\
$\mathcal{K}$ & $\frac{k_{e} q_{\text {ref }}^{2} t_{\text {ref }}}{m_{\text {ref }} v_{\text {ref }} l_{\text {ref }}^{2}}$ & $0.4,1.0,5.0$ \\
\hline
\end{tabular}

Table 5.2: Simulation parameters used in chapter 5 


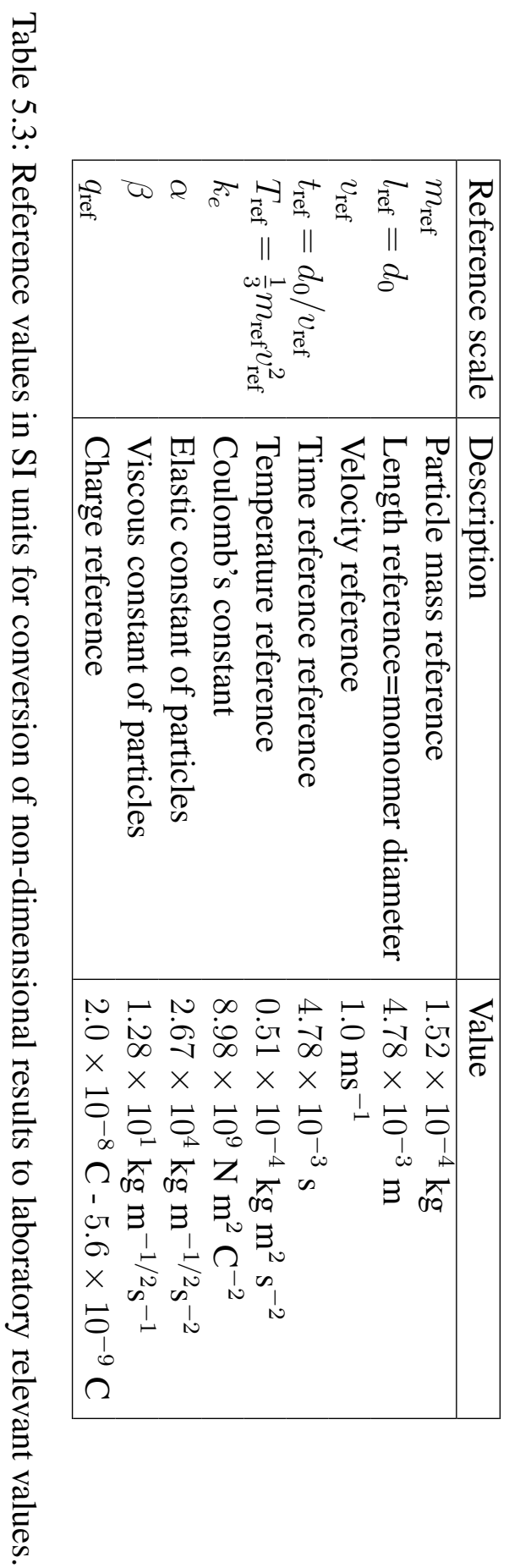




\section{Chapter 6}

\section{There is more happening before}

\section{aggregation}

In this chapter, the onset of clustering instability in charged granular gases is studied. A hydrodynamic description is presented, coupled to the electrostatic limit of Maxwell's equations. The regime under focus is where $\frac{k_{e} q_{\mathrm{ref}}^{2}}{\bar{r}} \ll T\left|\epsilon^{2}-1\right|$, i.e., the characteristic kinetic energy of the system, multiplied by the fractional energy lost per collision, is much higher than the characteristic electrostatic energy in the system. In this limit, it is assumed that the hydrodynamic transport coefficients remain the same as that of a neutral granular gas. A linear stability analysis indicates that at the onset, electrostatics tend to suppress suppress the so called "heat mode" of clustering instability, and might result in excitation of the "sound modes". It is shown that the "sound modes" become the dominant mechanism at the onset as the ratio $\frac{1}{\left|\epsilon^{2}-1\right|} \cdot \frac{q_{\mathrm{ref}}^{2} k_{e}}{\bar{r}}$ is increased. Finally, it is discussed that the electrostatic origin of instability, in principle, is non-linear in certain spatial regions of the granular gas, and the linear stability analysis presented in the chapter is valid away from these regions. 


\subsection{Dimensional analysis}

The granular gases are characterized by the fact that particle collisions are inelastic. The dissipation leads to a clustering instability which develops when the characteristic length scale over which the energy is dissipated becomes smaller than the characteristic length scale of density fluctuations (Goldhirsch \& Zanetti, 1993). This can be understood by the following arguments based on dimensional analysis.

Let us consider that the characteristic length scale of density fluctuations is $\ell_{\rho}$. The characteristic scale for speed in the granular gas might be taken equal to $\sqrt{T / m_{0}}$, where $T$ is the granular temperature and $m_{0}$ is the mass of a particle. Then a characteristic time scale $\tau_{\rho}$ can be defined over which the density fluctuations develop

$$
\tau_{\rho} \sim \frac{\ell_{\rho}}{\sqrt{T / m_{0}}}
$$

To compare it with dissipation, let us first consider the magnitude of energy loss $|\Delta E|$ in a single collision. If $v_{i j}$ is the magnitude of the relative velocity before a collision between two particles $i$ and $j$, then $|\Delta E| \sim \frac{1}{2} m_{0} v_{i j}^{2}\left|\epsilon^{2}-1\right|$, where $\epsilon$ is the coefficient of restitution. Or alternatively, the fractional energy lost in one collision is $\frac{|\Delta E|}{\frac{1}{2} m_{0} v_{i j}^{2}} \sim\left|\epsilon^{2}-1\right|$. If $\nu$ are the number of collisions taking place per unit volume and per unit time (i.e. collision frequency) in the gas with number density $n$, the the fractional energy lost per unit time per particle $\sim \nu\left|\epsilon^{2}-1\right| / n$. The inverse of this quantity can be chosen as the time scale (say $\tau_{\Delta E}$ ) of fractional energy loss per particle, i.e.

$$
\tau_{\Delta E} \sim \frac{n}{\nu\left|\epsilon^{2}-1\right|}
$$

If the time scale of fractional energy loss per particle is less than the time scale over which the density fluctuations develop, then one finds

$$
\ell_{\rho}>\frac{n \sqrt{T / m_{0}}}{\nu\left|\epsilon^{2}-1\right|}=\frac{\bar{r}}{\left|\epsilon^{2}-1\right|}
$$

where $\bar{r}$ is the mean free path of the particles. If $\epsilon \rightarrow 1$, the right hand side goes to $\infty$, and it becomes harder for the clustering instability to occur in a finite size system. The opposite is true if $\epsilon<1$. 
Now consider a granular gas with a distribution of charges. If $q_{\text {ref }}$ is the standard deviation of the charge distribution, then the characteristic time scale for two oppositely charged particles, separated by $\bar{r}$, to collapse under Coulomb potential can be chosen as

$$
\tau_{q} \sim \frac{\bar{r}^{2} \sqrt{m_{0} T}}{k_{e} q_{\mathrm{ref}}^{2}}
$$

where $k_{e}$ is Coulomb's constant. If this time scale is smaller than $\tau_{\rho}$, one finds

$$
\ell_{\rho}>\left(\frac{\bar{r}}{q_{\mathrm{ref}} \sqrt{k_{e} / T}}\right)^{2}
$$

In this case, it becomes harder for the system to develop density fluctuations as $q_{\text {ref }} \sqrt{k_{e} / T} \rightarrow 0$. From Eq. (6.3) and (6.5), it follows that in a charged granular gas, the dissipation would be the dominant mechanism behind the growth of density fluctuations, if

$$
\frac{\bar{r}}{\left|\epsilon^{2}-1\right|}<\ell_{\rho}<\left(\frac{\bar{r}}{q_{\mathrm{ref}} \sqrt{k_{e} / T}}\right)^{2}
$$

or if

$$
\frac{k_{e} q_{\mathrm{ref}}^{2}}{\bar{r}} \ll T\left|\epsilon^{2}-1\right|
$$

For a given charge distribution, the above condition is less stringent for a dilute granular gas (larger $\bar{r}$ ) with smaller $\epsilon$.

In the following, this competition between dissipation and electrostatics is shown quantitatively with the help of linear stability analysis of the granular hydrodynamic equations.

\subsection{Hydrodynamics in the limit $\frac{k_{e} q_{\mathrm{ref}}^{2}}{\bar{r}} \ll T\left|\epsilon^{2}-1\right|$}

In this and the next sections, the mechanisms of the onset of instability in the context of macroscopic fields is studied in the regime $\frac{k_{e} q_{\text {ref }}^{2}}{\bar{r}} \ll T\left|\epsilon^{2}-1\right|$. This limit essentially states that the characteristic kinetic energy, multiplied by the fractional energy lost per collision, is much higher in comparison to the characteristic Coulomb energy in the system. The gas has not yet cooled down to a state where the long-range forces are capable of any aggregative collapse. Under this ansatz, it can be assumed that the kinetic coefficients 
retain their forms similar to that of a neutral granular gas, while the effect of electrostatics is only perturbative. It is assumed that the coefficient of restitution $\epsilon$ is constant. First, the hydrodynamic equations for the mass, momentum, energy and charge transport, coupled to the mean electrostatic field are described. Thereafter the linear stability analysis of the hydrodynamic equations is presented.

\subsubsection{Mass transport}

Let us first consider the mass balance. Multiplying the Boltzmann equation from chapter 2 with particle mass $m$, and integrating over the phase space variables $\mathbf{v}, q$, one obtains

$$
\int m \frac{\partial f}{\partial t} d \mathbf{v} d q+\int m \frac{\partial}{\partial \mathbf{r}} \cdot \mathbf{v} f d \mathbf{v} d q+\int m \frac{\partial}{\partial \mathbf{v}} \cdot \frac{q \mathbf{E}}{m} f d \mathbf{v} d q=\int m I^{\mathrm{coll}} d \mathbf{v} d q
$$

In the following, it is assumed that the phase space variables $\mathbf{r}, \mathbf{v}$ and $q$ are independent of each other. Using the definitions of number density $n$ and the macroscopic velocity $\mathbf{V}$ from chapter 2 , the above equation provides

$$
m \frac{\partial n}{\partial t}+m \frac{\partial}{\partial \mathbf{r}} \cdot n \mathbf{V}+\int \frac{\partial}{\partial \mathbf{v}} \cdot q \mathbf{E} f d \mathbf{v} d q=0
$$

The collision term on the right hand side vanishes due to the mass conservation during collisions. The integral over $\mathbf{v}$ in third term on the left hand side can be transformed to a surface integral in the velocity space, i.e. $\int \frac{\partial}{\partial \mathbf{v}} \cdot \mathbf{E} f d \mathbf{v}=\int(\mathbf{E} f) \cdot d \mathbf{A}_{\mathbf{v}}$, which also vanishes. Thus the mass balance results in

$$
\frac{\partial \rho}{\partial t}+\frac{\partial}{\partial \mathbf{r}} \cdot \rho \mathbf{V}=0
$$

where $\rho=m n$ is the mass density in the gas. 


\subsubsection{Momentum transport}

Multiplying the Boltzmann equation with particle momentum $m \mathbf{v}$, and integrating over the phase space variables $\mathbf{v}, q$, one obtains

$$
\int m \mathbf{v} \frac{\partial f}{\partial t} d \mathbf{v} d q+\int m \mathbf{v} \frac{\partial}{\partial \mathbf{r}} \cdot \mathbf{v} f d \mathbf{v} d q+\int m \mathbf{v} \frac{\partial}{\partial \mathbf{v}} \cdot \frac{q \mathbf{E}}{m} f d \mathbf{v} d q=\int m \mathbf{v} I^{\text {coll }} d \mathbf{v} d q
$$

The first term simplify as $\int m \mathbf{v} \frac{\partial f}{\partial t} d \mathbf{v} d q=m \frac{\partial}{\partial t} \int \mathbf{v} f d \mathbf{v} d q=\frac{\partial}{\partial t} \rho \mathbf{V}$. In the second term, the particle microscopic velocities $\mathbf{v}$ are first considered as the sum of the macroscopic velocities $\mathbf{V}$ and a fluctuating part $\mathbf{v}^{\prime}$. This leads to $\mathbf{v} \mathbf{v}=\left(\mathbf{V}+\mathbf{v}^{\prime}\right)\left(\mathbf{V}+\mathbf{v}^{\prime}\right)=\mathbf{V} \mathbf{V}+$ $\mathbf{v}^{\prime} \mathbf{v}^{\prime}+2 \mathbf{V} \mathbf{v}^{\prime}$. Here the notation $\mathbf{v} \mathbf{v} \equiv \mathbf{v} \otimes \mathbf{v} \equiv v_{i} v_{j}$ denotes a tensor product. Thus the integral in the second term equals the sum

$$
\int m \frac{\partial}{\partial \mathbf{r}} \cdot \mathbf{V} \mathbf{V} f d \mathbf{v} d q+\int m \frac{\partial}{\partial \mathbf{r}} \cdot \mathbf{v}^{\prime} \mathbf{v}^{\prime} f d \mathbf{v} d q+\int m \frac{\partial}{\partial \mathbf{r}} \cdot 2 \mathbf{V} \mathbf{v}^{\prime} f d \mathbf{v} d q
$$

The first term in the above sum simplyfies as $\int m \frac{\partial}{\partial \mathbf{r}} \cdot \mathbf{V} \mathbf{V} f d \mathbf{v} d q=\frac{\partial}{\partial \mathbf{r}} \cdot m \mathbf{V V} \int f d \mathbf{v} d q=$ $\frac{\partial}{\partial \mathbf{r}} \cdot \rho \mathbf{V V}$. The second term in the sum is rewritten as

$$
\int m \frac{\partial}{\partial \mathbf{r}} \cdot \mathbf{v}^{\prime} \mathbf{v}^{\prime} f d \mathbf{v} d q=\frac{\partial}{\partial \mathbf{r}} \cdot \int m \mathbf{v}^{\prime} \mathbf{v}^{\prime} f d \mathbf{v} d q=\frac{\partial}{\partial \mathbf{r}} \cdot \overline{\mathbf{P}}
$$

where $\overline{\mathbf{P}}=\int m \mathbf{v}^{\prime} \mathbf{v}^{\prime} f d \mathbf{v} d q$ is the kinetic part of the pressure tensor. The last term in the sum vanishes as follows

$$
2 m \frac{\partial}{\partial \mathbf{r}} \cdot \mathbf{V} \int \mathbf{v}^{\prime} f d \mathbf{v} d q=2 m \frac{\partial}{\partial \mathbf{r}} \cdot \mathbf{V} \int(\mathbf{v}-\mathbf{V}) f d \mathbf{v} d q=2 m \frac{\partial}{\partial \mathbf{r}} \cdot \mathbf{V}(n \mathbf{V}-n \mathbf{V})=0
$$

The term $\int m \mathbf{v} I^{\text {coll }} d \mathbf{v} d q$ in Eq. (6.11) also vanishes due to momentum conservation during collisions, while the term $\int m \mathbf{v} \frac{\partial}{\partial \mathbf{v}} \cdot \frac{q \mathbf{E}}{m} f d \mathbf{v} d q$ reduces to $-n Q \mathbf{E}$. Here the definition of the mean or macroscopic charge $Q$ is used from chapter 2. Thus the momentum balance provides

$$
\frac{\partial}{\partial t} \rho \mathbf{V}+\frac{\partial}{\partial \mathbf{r}} \cdot \rho \mathbf{V} \mathbf{V}=-\frac{\partial}{\partial \mathbf{r}} \cdot \overline{\mathbf{P}}+n Q \mathbf{E}
$$




\subsubsection{Kinetic energy transport}

Multiplying the Boltzmann equation with $\frac{1}{2} m(\mathbf{v}-\mathbf{V})^{2}=\frac{1}{2} m \mathbf{v}^{\prime 2}$, and integrating over the phase space variables $\mathbf{v}, q$, one obtains

$$
\begin{aligned}
\int \frac{1}{2} m \mathbf{v}^{\prime 2} \frac{\partial f}{\partial t} d \mathbf{v} d q & +\int \frac{1}{2} m \mathbf{v}^{\prime 2} \frac{\partial}{\partial \mathbf{r}} \cdot \mathbf{v} f d \mathbf{v} d q+\int \frac{1}{2} m \mathbf{v}^{\prime 2} \frac{\partial}{\partial \mathbf{v}} \cdot \frac{q \mathbf{E}}{m} f d \mathbf{v} d q \\
& =\int \frac{1}{2} m \mathbf{v}^{\prime 2} I^{\text {coll }} d \mathbf{v} d q
\end{aligned}
$$

The first term, according to the definition of granular temperature from chapter 2 , is simply $\frac{\partial}{\partial t} \frac{3}{2} n T$. The second term, again using $\mathbf{v}=\mathbf{V}+\mathbf{v}^{\prime}$, and after some manipulations ${ }^{1}$ provides

$$
\int \frac{1}{2} m \mathbf{v}^{\prime 2} \frac{\partial}{\partial \mathbf{r}} \cdot \mathbf{v} f d \mathbf{v} d q=\frac{\partial}{\partial \mathbf{r}} \cdot \frac{3}{2} n T \mathbf{V}+\frac{\partial}{\partial \mathbf{r}} \cdot \mathbf{Q}+\overline{\mathbf{P}}: \frac{\partial}{\partial \mathbf{r}} \mathbf{V}
$$

where $\mathrm{Q}$ is the heat flux vector, and the notation ":" represents a tensor contraction. The term due to electric field in Eq. (6.16) takes the form $\int \frac{1}{2} m \mathbf{v}^{\prime 2} \frac{\partial}{\partial \mathbf{v}} \cdot \frac{q \mathbf{E}}{m} f d \mathbf{v} d q$ reduced to $-n Q \mathbf{E} \cdot \mathbf{V}$, while the collision term provides

$$
\int \frac{1}{2} m \mathbf{v}^{\prime 2} I^{\text {coll }} d \mathbf{v} d q=-\frac{3}{2} n T \zeta
$$

Here $\zeta$ is the cooling coefficient. Thus finally the energy transport is written as

$$
\frac{\partial}{\partial t} \frac{3}{2} n T+\frac{\partial}{\partial \mathbf{r}} \cdot \frac{3}{2} n T \mathbf{V}=-\frac{\partial}{\partial \mathbf{r}} \cdot \mathbf{Q}-\overline{\mathbf{P}}: \frac{\partial}{\partial \mathbf{r}} \mathbf{V}-\frac{3}{2} n T \zeta+n Q \mathbf{E} \cdot \mathbf{V}
$$

Again, the notation ":" represents a tensor contraction.

\subsubsection{Charge transport}

Multiplying the Boltzmann equation with particle charge $q$, and integrating over the phase space variables $\mathbf{v}, q$, one obtains

$$
\int q \frac{\partial f}{\partial t} d \mathbf{v} d q+\int q \frac{\partial}{\partial \mathbf{r}} \cdot \mathbf{v} f d \mathbf{v} d q+\int q \frac{\partial}{\partial \mathbf{v}} \cdot \frac{q \mathbf{E}}{m} f d \mathbf{v} d q=\int q I^{\mathrm{coll}} d \mathbf{v} d q
$$

\footnotetext{
${ }^{1}$ See, for example, Pitaevskii \& Lifshitz (2012).
} 
The first term provide $\int q \frac{\partial f}{\partial t} d \mathbf{v} d q=\frac{\partial}{\partial t} \int q f d \mathbf{v} d q=\frac{\partial}{\partial t} n Q$. The second term is first rewritten as

$$
\int q \frac{\partial}{\partial \mathbf{r}} \cdot \mathbf{v} f d \mathbf{v} d q=\frac{\partial}{\partial \mathbf{r}} \cdot \int q \mathbf{v} f d \mathbf{v} d q=\frac{\partial}{\partial \mathbf{r}} \cdot \int\left(Q+q^{\prime}\right)\left(\mathbf{V}+\mathbf{v}^{\prime}\right) f d \mathbf{v} d q
$$

where the particle velocity and charge is written as the sum of their mean values $\mathbf{V}$ and $Q$, and corresponding fluctuating parts $\mathbf{v}^{\prime}$, and $q^{\prime}$. The integral then splits as

$$
\begin{aligned}
\frac{\partial}{\partial \mathbf{r}} \cdot \int\left(Q+q^{\prime}\right)\left(\mathbf{V}+\mathbf{v}^{\prime}\right) f d \mathbf{v} d q= & \frac{\partial}{\partial \mathbf{r}} \cdot Q \mathbf{V} \int f d \mathbf{v} d q \\
& +\frac{\partial}{\partial \mathbf{r}} \cdot Q \int \mathbf{v}^{\prime} f d \mathbf{v} d q \\
& +\frac{\partial}{\partial \mathbf{r}} \cdot \mathbf{V} \int q^{\prime} f d \mathbf{v} d q \\
& +\frac{\partial}{\partial \mathbf{r}} \cdot \int \mathbf{v}^{\prime} q^{\prime} f d \mathbf{v} d q
\end{aligned}
$$

The first integral on the right hand side provides $\frac{\partial}{\partial \mathbf{r}} \cdot Q \mathbf{V} \int f d \mathbf{v} d q=\frac{\partial}{\partial \mathbf{r}} \cdot n Q \mathbf{V}$. The second, and the third integral vanishes following arguments similar to Eq. (6.14). The last integral provides a term, $n\left\langle\mathbf{v}^{\prime} q^{\prime}\right\rangle$, consisting of correlations between charge and velocity fluctuations. Here it is assumed that the charge and velocity fluctuations are uncorrelated, and the last integral is neglected. The third term on left hand side of Eq. (6.19) again vanishes. The charge during collisions is assumed to be conserved, therefore, the collision term also vanishes, resulting in the charge transport equation

$$
\frac{\partial \rho_{\mathrm{q}}}{\partial t}+\frac{\partial}{\partial \mathbf{r}} \cdot \rho_{\mathrm{q}} \mathbf{V}=0
$$

where $\rho_{\mathrm{q}}=n Q$ is the charge density.

The above transport equations are to be coupled to the electrostatic limit of Maxwell's equations for the mean electric field $\mathbf{E}$, which are

$$
\frac{\partial}{\partial \mathbf{r}} \cdot \mathbf{E}=4 \pi \rho_{\mathrm{q}}, \frac{\partial}{\partial \mathbf{r}} \times \mathbf{E}=0
$$

Using $\mathbf{E}=\frac{\partial}{\partial \mathbf{r}} \varphi$ as a result from the second equation, the above two equations can be 
reduced to a single equation for the electrostatic potential $\varphi$, written as

$$
\frac{\partial^{2}}{\partial \mathbf{r}^{2}} \varphi=4 \pi \rho_{\mathrm{q}}
$$

\subsubsection{Pressure tensor, heat flux, and transport coefficients}

For a granular gas, the pressure tensor $\overline{\mathbf{P}} \equiv P_{i j}$ and the heat flux vector $\mathbf{Q}$ have the form (Brilliantov \& Pöschel, 2010)

$$
\begin{aligned}
P_{i j} & =p \delta_{i j}-\eta\left[\frac{\partial V_{j}}{\partial r_{i}}+\frac{\partial V_{i}}{\partial r_{j}}-\frac{2}{3} \delta_{i j} \frac{\partial}{\partial \mathbf{r}} \cdot \mathbf{V}\right] \\
\mathbf{Q} & =-\kappa \frac{\partial T}{\partial \mathbf{r}}-\mu \frac{\partial n}{\partial \mathbf{r}}
\end{aligned}
$$

In the above expressions, $p$ is the hydrostatic pressure, $\kappa$ is the thermal conductivity, and $\eta$ is the shear viscosity. The coefficient $\mu$ appear only for granular gases (Brilliantov \& Pöschel, 2010) and does not have a counterpart for molecular fluids. It relates the heat flux to the density gradient. The coefficients $\eta, \mu, \kappa$ are obtained from the microscopic properties of the particles using either Chapman-Enskog approach or Grads method, for example by Brilliantov \& Pöschel (2010). In the following section, the linear stability of the macroscopic equations is presented, for a time regime where $\frac{1}{\left|\epsilon^{2}-1\right|} \cdot \frac{q_{\mathrm{ref}}^{2} k_{e}}{\bar{r}} \ll T$, and the variations in the transport coefficients due to electrostatic field are neglected.

\subsection{Linear stability of the hydrodynamic equations}

Taken together, the hydrodynamic equations are written into the following form

$$
\begin{aligned}
\frac{\partial \rho}{\partial t} & =-\frac{\partial}{\partial \mathbf{r}} \cdot \rho \mathbf{V} \\
\frac{\partial \mathbf{V}}{\partial t} & =-\mathbf{V} \cdot \frac{\partial}{\partial \mathbf{r}} \mathbf{V}-\frac{1}{\rho} \frac{\partial p}{\partial \mathbf{r}}+\frac{\eta}{\rho}\left[\frac{\partial^{2}}{\partial \mathbf{r}^{2}} \mathbf{V}+\frac{1}{3} \frac{\partial}{\partial \mathbf{r}}\left(\frac{\partial}{\partial \mathbf{r}} \cdot \mathbf{V}\right)\right]+\frac{\rho_{\mathrm{q}}}{\rho} \nabla \varphi \\
\frac{\partial T}{\partial t} & =-\mathbf{V} \cdot \frac{\partial}{\partial \mathbf{r}} T+\frac{2}{3 n}\left[\kappa \frac{\partial^{2}}{\partial \mathbf{r}^{2}} T+\mu \frac{\partial^{2}}{\partial \mathbf{r}^{2}} n\right]-\frac{2}{3 n} \overline{\mathbf{P}}: \frac{\partial}{\partial \mathbf{r}} \mathbf{V}-T \zeta+\frac{2}{3 n} \rho_{\mathrm{q}} \nabla \varphi \cdot \mathbf{V} \\
\frac{\partial \rho_{\mathrm{q}}}{\partial t} & =-\frac{\partial}{\partial \mathbf{r}} \cdot \rho_{\mathrm{q}} \mathbf{V} \\
\frac{\partial^{2}}{\partial \mathbf{r}^{2}} \varphi & =4 \pi \rho_{\mathrm{q}} .
\end{aligned}
$$


The fields $\rho, \mathbf{V}, T, \rho_{\mathrm{q}}$ and $\varphi$ are then perturbed around a state which is homogeneous with zero mean velocity, i.e.

$$
\begin{aligned}
\rho(\mathbf{r}, t) & =\rho_{0}+\delta \rho(\mathbf{r}, t), \\
\mathbf{V}(\mathbf{r}, t) & =\mathbf{0}+\delta \mathbf{V}(\mathbf{r}, t), \\
T(\mathbf{r}, t) & =T_{0}(t)+\delta T(\mathbf{r}, t), \\
\rho_{\mathrm{q}}(\mathbf{r}, t) & =\rho_{\mathrm{q} 0}+\delta \rho_{\mathrm{q}}(\mathbf{r}, t), \\
\varphi(\mathbf{r}, t) & =\varphi_{0}+\delta \varphi(\mathbf{r}, t) .
\end{aligned}
$$

For the sake of the linear stability analysis, it is assumed that the transport coefficients are space independent. The fields are rescaled using $\tilde{\rho}=\rho / \rho_{0}, \tilde{\mathbf{V}}=\mathbf{V} / v_{\operatorname{th}}(t), \tilde{T}=T / T_{0}(t)$, $\tilde{\rho}_{\mathrm{q}}=\rho_{\mathrm{q}} / \rho_{\mathrm{q} 0}, \tilde{t}=t / t_{0}(t), \tilde{\mathbf{r}}=\mathbf{r} / \ell_{0}, \tilde{\zeta}=t_{0} \zeta$ and $\tilde{\varphi}=\varphi / \varphi_{0}=\varphi /\left(4 \pi \rho_{\mathrm{q} 0} \ell_{0}^{2}\right)$, where $v_{\mathrm{th}}(t)=\sqrt{2 T_{0}(t) / m}=\ell_{0} / t_{0}(t) .{ }^{1} \quad$ Then the scaled perturbation expansions around the homogeneous state read as

$$
\begin{gathered}
\tilde{\rho}(\mathbf{r}, t)=1+\delta \tilde{\rho}(\mathbf{r}, t), \\
\tilde{\mathbf{V}}(\mathbf{r}, t)=\mathbf{0}+\delta \tilde{\mathbf{V}}(\mathbf{r}, t), \\
\tilde{T}(\mathbf{r}, t)=1+\delta \tilde{T}(\mathbf{r}, t), \\
\tilde{\rho_{\mathrm{q}}}(\mathbf{r}, t)=1+\delta \tilde{\rho_{\mathrm{q}}}(\mathbf{r}, t), \\
\tilde{\varphi}(\mathbf{r}, t)=1+\delta \tilde{\varphi}(\mathbf{r}, t) .
\end{gathered}
$$

For the scaled perturbations, it is assumed that $\delta \tilde{\rho} \ll 1, \delta \tilde{T} \ll 1, \delta \tilde{\rho_{\mathrm{q}}} \ll 1, \delta \tilde{\varphi} \ll 1$, and $|\delta \tilde{\mathbf{V}}| \ll 1$. Also using these scales, Eqs. (6.26) can be non-dimensionalized. Putting Eqs. (6.28) into non-dimensionalized hydrodynamic equations, and preserving only the

\footnotetext{
${ }^{1}$ Notice that here the time scale $t_{0}(t)$ is itself time dependent. See Brilliantov \& Pöschel (2010) for details on this choice.
} 
terms which are linear in the perturbations, one finds

$$
\begin{aligned}
\frac{\partial \delta \tilde{\rho}}{\partial \tilde{t}} & =-\frac{\partial}{\partial \tilde{\mathbf{r}}} \cdot \delta \tilde{\mathbf{V}} \\
\frac{\partial \delta \tilde{\mathbf{V}}}{\partial \tilde{t}} & =\frac{1}{4} \zeta^{*} \delta \tilde{\mathbf{V}}-\frac{1}{2}\left[\frac{\partial}{\partial \tilde{\mathbf{r}}} \delta \tilde{\rho}+\frac{\partial}{\partial \tilde{\mathbf{r}}} \delta \tilde{T}\right]+\eta^{*}\left[\frac{\partial^{2}}{\partial \tilde{\mathbf{r}}^{2}} \delta \tilde{\mathbf{V}}+\frac{1}{3} \frac{\partial}{\partial \tilde{\mathbf{r}}}\left(\frac{\partial}{\partial \tilde{\mathbf{r}}} \cdot \delta \tilde{\mathbf{V}}\right)\right]+\mathcal{R} \frac{\partial}{\partial \tilde{\mathbf{r}}} \delta \tilde{\varphi} \\
\frac{\partial \delta \tilde{T}}{\partial \tilde{t}} & =-\frac{1}{4} \zeta^{*} \delta \tilde{T}-\frac{1}{2} \zeta^{*} \delta \tilde{\rho}+\frac{5}{2}\left[\kappa^{*} \frac{\partial^{2}}{\partial \tilde{\mathbf{r}}^{2}} \delta \tilde{T}+\mu^{*} \frac{\partial^{2}}{\partial \tilde{\mathbf{r}}^{2}} \delta \tilde{\rho}\right]-\frac{2}{3}\left[\frac{\partial}{\partial \tilde{\mathbf{r}}} \cdot \delta \tilde{\mathbf{V}}\right] \\
\frac{\partial \delta \tilde{\rho_{\mathrm{q}}}}{\partial \tilde{t}} & =-\frac{\partial}{\partial \tilde{\mathbf{r}}} \cdot \delta \tilde{\mathbf{V}} \\
\frac{\partial^{2}}{\partial \tilde{\mathbf{r}}^{2}} \delta \tilde{\varphi} & =1+\delta \tilde{\rho_{\mathrm{q}}} .
\end{aligned}
$$

The rescaled transport coefficient are now pure numbers (Brilliantov \& Pöschel, 2010), and their relation with the coefficient of restitution is provided in appendix 3 . The nondimensional number $\mathcal{R}$ is

$$
\mathcal{R}=\frac{2 \pi q_{\mathrm{ref}}^{2}}{T_{0} \ell_{0}} \frac{\rho_{0} t_{0} \ell_{0}^{2}}{m_{0}} \propto \mathcal{B}
$$

The scaled perturbation equations are then transformed into Fourier space. If $\mathbf{a}(\mathbf{r}, t)$ is a field in $\mathbf{r}$ space, then

$$
\mathbf{a}_{k}(t)=\frac{1}{\sqrt{L^{3}}} \int e^{-i \mathbf{k} \cdot \mathbf{r}} \mathbf{a}(\mathbf{r}, t) d \mathbf{r}
$$

where $\mathbf{k}=2 \pi \mathbf{b} / L$, with $\mathbf{b}=\left(b_{x}, b_{y}, b_{z}\right)$ being a vector of integers $b_{x}, b_{y}, b_{z}$. The transform provides the following scaled perturbation equations in $\mathbf{k}$ space

$$
\begin{aligned}
\frac{\partial \delta \tilde{\rho}_{k}}{\partial \tilde{t}} & =-i \mathbf{k} \cdot \delta \tilde{\mathbf{V}}_{k} \\
\frac{\partial \delta \tilde{\mathbf{V}}_{k}}{\partial \tilde{t}} & =\frac{1}{4} \zeta^{*} \delta \tilde{\mathbf{V}}_{k}-\frac{1}{2} i \mathbf{k}\left[\delta{\tilde{\rho_{k}}}+\delta \tilde{T}_{k}\right]+\eta^{*}\left[(i \mathbf{k})^{2} \delta \tilde{\mathbf{V}}_{k}+\frac{1}{3} i \mathbf{k}\left(i \mathbf{k} \cdot \delta \tilde{\mathbf{V}}_{k}\right)\right]+\mathcal{R} i \mathbf{k} \delta \tilde{\varphi}_{k}, \\
\frac{\partial \delta \tilde{T}_{k}}{\partial \tilde{t}} & =-\frac{1}{4} \zeta^{*} \delta \tilde{T}_{k}-\frac{1}{2} \zeta^{*} \delta \tilde{\rho}_{k}+\frac{5}{2}\left[\kappa^{*}(i \mathbf{k})^{2} \delta \tilde{T}_{k}+\mu^{*}(i \mathbf{k})^{2} \delta \tilde{\rho}_{k}\right]-\frac{2}{3}\left[i \mathbf{k} \cdot \delta \tilde{\mathbf{V}}_{k}\right] \\
\frac{\partial \delta \tilde{\rho}_{q, k}}{\partial \tilde{t}} & =-i \mathbf{k} \cdot \delta \tilde{\mathbf{V}}_{k}, \\
(i \mathbf{k})^{2} \delta \tilde{\varphi}_{k} & =\delta \tilde{\rho}_{q, k} .
\end{aligned}
$$


In the last equation it is assumed that $\mathbf{k} \neq \mathbf{0}$. The last two equations in the above set can be combined, and the time derivative of $\delta \tilde{\varphi_{k}}$ can be obtained as

$$
\frac{\partial \delta \tilde{\varphi}_{k}}{\partial \tilde{t}}=\frac{1}{k^{2}} i \mathbf{k} \cdot \delta \tilde{\mathbf{V}}_{k}
$$

In shorthand, the perturbation equations can be expressed by

$$
\frac{\partial \delta \tilde{\boldsymbol{\psi}}_{k}}{\partial \tilde{t}}=\overline{\mathbf{M}} \delta \tilde{\boldsymbol{\psi}}_{k}
$$

where $\delta \tilde{\boldsymbol{\psi}}_{k}=\left(\delta \tilde{\rho}_{k}, \delta \tilde{T}_{k}, \delta \tilde{\mathbf{V}}_{k}, \delta \tilde{\varphi}_{k}\right)$, and $\overline{\mathbf{M}}$ is a matrix containing coefficients from the right hand sides of Eq. (6.32) and (6.33). The eigenvectors of $\overline{\mathbf{M}}$ are defined by the equation

$$
\overline{\mathbf{M}} \delta \tilde{\boldsymbol{\psi}}_{k, l}=\lambda_{l} \delta \tilde{\boldsymbol{\psi}}_{k, l}
$$

These eigenvectors, also called hydrodynamic modes, are decoupled from each other in the linearized framework and evolve independently. Their time evolution is given by

$$
\delta \tilde{\boldsymbol{\psi}}_{k, l}(\tilde{t})=\delta \tilde{\boldsymbol{\psi}}_{k, l}(0) \exp \left[\lambda_{l} \tilde{t}\right]
$$

If the real part of the eigenvalues $\lambda_{l} \leq 0$, the concerned modes are stable, that is small initial perturbations decay exponentially in time; if $\lambda_{l}>0$, the corresponding modes are unstable. Moreover, the scaled velocity perturbation can be written as a sum of its two components, one chosen perpendicular, and the other parallel to the vector $\mathrm{k}$, i.e.

$$
\begin{aligned}
\delta \tilde{\mathbf{V}}_{k \|} & =\left(\delta \tilde{\mathbf{V}}_{k} \cdot \mathbf{k}\right) \frac{\mathbf{k}}{k}=\delta \tilde{V}_{k \|} \frac{\mathbf{k}}{k} \\
\delta \tilde{\mathbf{V}}_{k \perp} & =\delta \tilde{\mathbf{V}}_{k}-\delta \tilde{\mathbf{V}}_{k \|} \cdot
\end{aligned}
$$

Below, the stability results for the transverse/perpendicular velocity perturbation, longitudinal/parallel velocity perturbation, and other hydrodynamic modes are discussed. 


\subsubsection{Transverse modes}

Using $\mathbf{k} \cdot \delta \tilde{\mathbf{V}}_{k \perp}=0$ in Eq. (6.32), the equation for the mode $\delta \tilde{\mathbf{V}}_{k \perp}$ is decoupled from other modes, and reads as

$$
\frac{\partial \delta \tilde{\mathbf{V}}_{k \perp}}{\partial \tilde{t}}=\left[\frac{1}{4} \zeta^{*}-\eta^{*} k^{2}\right] \delta \tilde{\mathbf{V}}_{k \perp}
$$

For the mode $\delta \tilde{\mathbf{V}}_{k \perp}$ to be unstable, the eigenvalue $\frac{1}{4} \zeta^{*}-\eta^{*} k^{2}$ should be positive, which requires $k<\sqrt{\zeta^{*} / 4 \eta^{*}}$.

The linear stability analysis thus depicts that electrostatics does not alter the evolution of these transverse modes. These modes lead to the formation of vortices in the granular gas and are also called shear modes. Thus the emergence of vortex formation in the linear regime in a charged granular gas is expected to be similar to a neutral granular gas.

\subsubsection{Longitudinal/parallel and other hydrodynamic modes}

Excluding the decoupled transverse mode, the matrix $\overline{\mathbf{M}}$ for the remaining modes $\delta \tilde{\boldsymbol{\psi}}_{k}=$ $\left(\delta \tilde{\rho}_{k}, \delta \tilde{T}_{k}, \delta \tilde{V}_{k \|}, \delta \tilde{\varphi}_{k}\right)$ now reads

$$
\overline{\mathbf{M}}=\left(\begin{array}{cccc}
0 & 0 & -i k & 0 \\
-\frac{1}{2} \zeta^{*}-\frac{5}{2} \mu^{*} k^{2} & -\frac{1}{4} \zeta^{*}-\frac{5}{2} \kappa^{*} k^{2} & -\frac{2}{3} i k & 0 \\
-\frac{1}{2} i k & -\frac{1}{2} i k & \frac{1}{4} \zeta^{*}-\frac{4}{3} \eta^{*} k^{2} & \mathcal{R} i k \\
0 & 0 & \frac{-1}{i k} & 0
\end{array}\right)
$$

The eigenvalues, $\lambda_{i}$, of the above matrix with respect to $k$ are plotted in Fig. 6.1 and 6.2 with varying values of $\epsilon$ and $\mathcal{R}$.

In Fig. 6.1, the value of $\epsilon=0.7$ is fixed while the non-dimensional number $\mathcal{R}$, which is proportional to the Bjerrum number $\mathcal{B}$ computed in chapter 5 , is varied from 0 to 0.9 . The case $\mathcal{R}=0$ [Fig. 6.1(a)] corresponds to a neutral granular gas (solid lines). Two of the non-zero eigenvalues of the matrix in Eq. (6.39) are $\leq 0$ for all $k$, while one of them can be positive up to a certain value of $k$. The unstable mode, in this case, is referred to as heat mode, while the other two stable modes are non-propagating sound modes (Brilliantov \& Pöschel, 2010). If the characteristic size $L$ of the system permits the existence of $k$ 


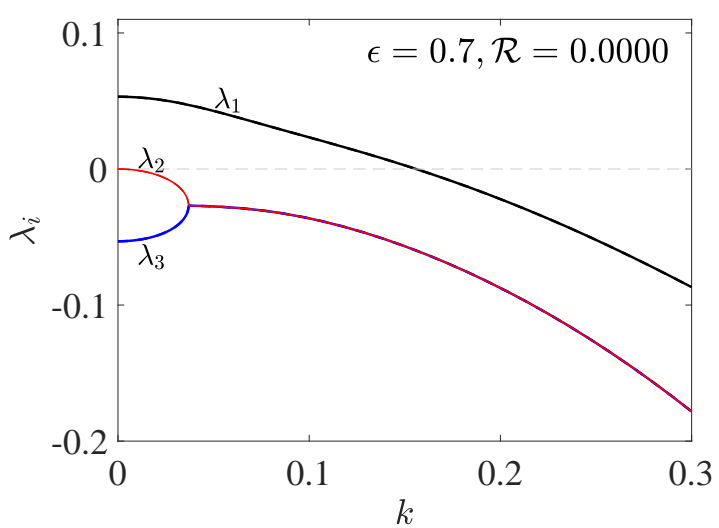

(a)

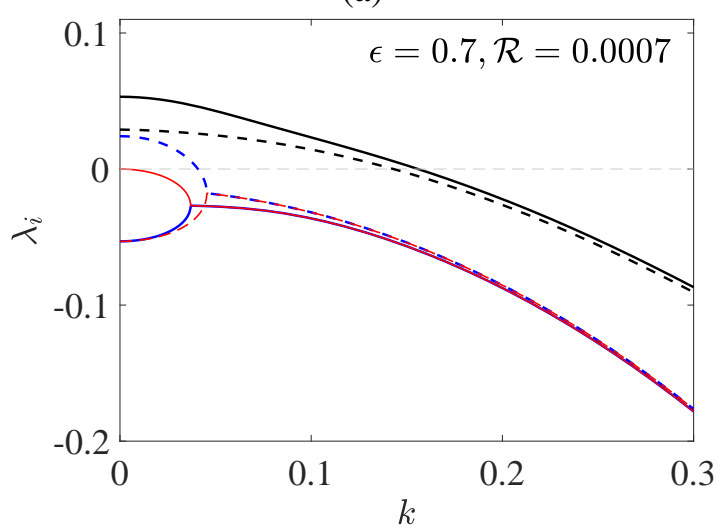

(c) $t=1.0$

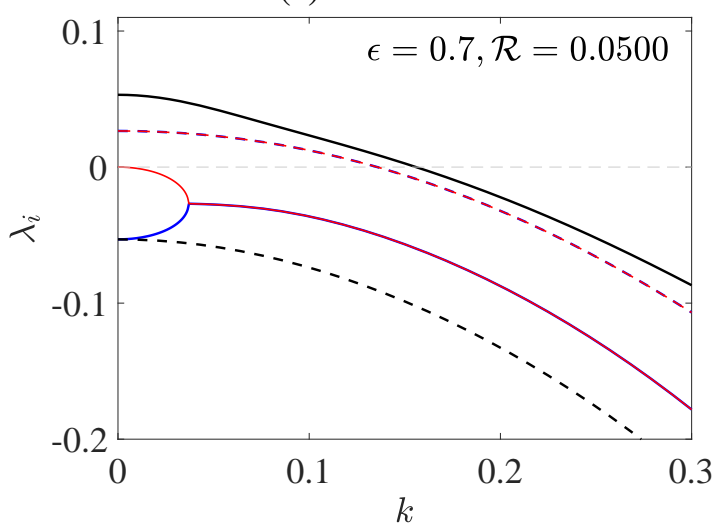

(e) $t=1.0$

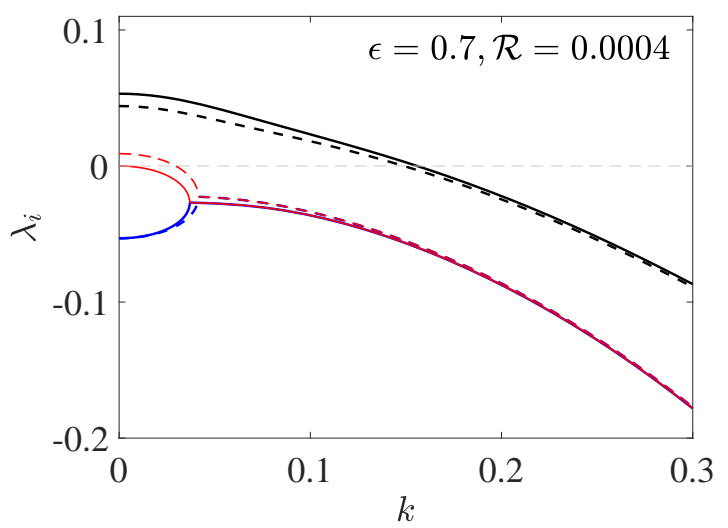

(b)

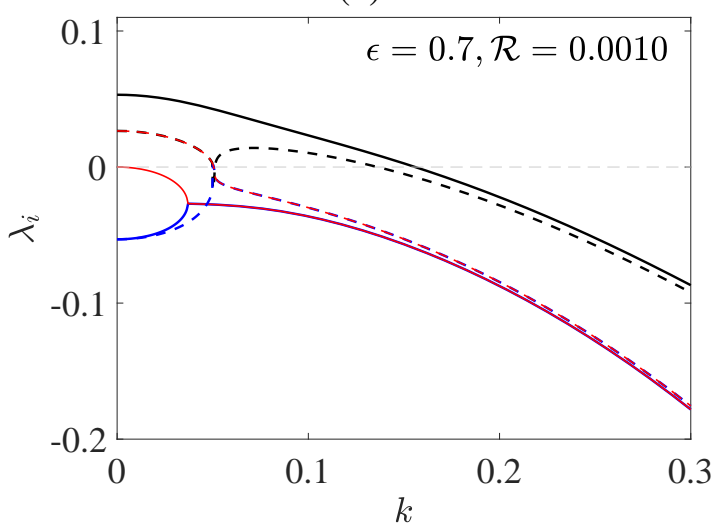

(d)

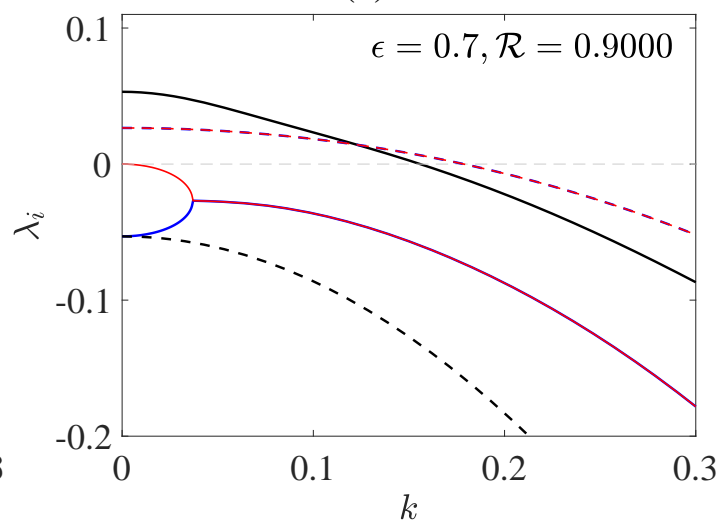

(f)

Figure 6.1: The change in the eigenvalues $\lambda_{i}(k)$ as the value of the non-dimensional parameter $\mathcal{R}$ is increased from 0 (a) to 0.9 (f). The case $\mathcal{R}=0$ corresponds to a neutral granular gas (solid lines), where one eigenvalue is positive and signifies heat mode of clustering instability. As $\mathcal{R}$ is increased, the eigenvalue corresponding to the heat mode is suppressed, and the other two eigenvalues may become equal and positive (dashed lines). At certain $\mathcal{R}$, these two eigenvalues take over [e.g. in subplot (e) and (f)]. In this regime, the heat mode now becomes stable. 


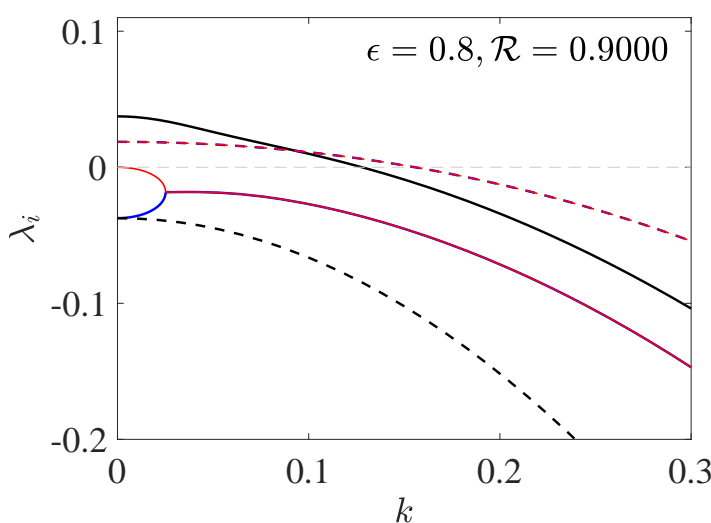

(a)

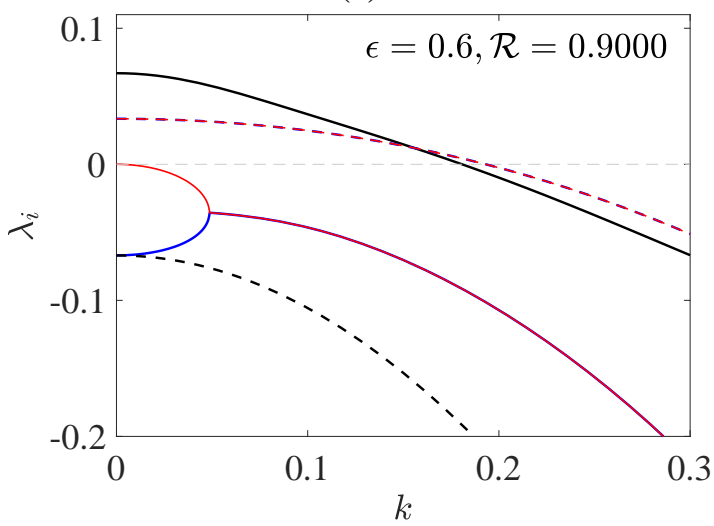

(c) $t=1.0$

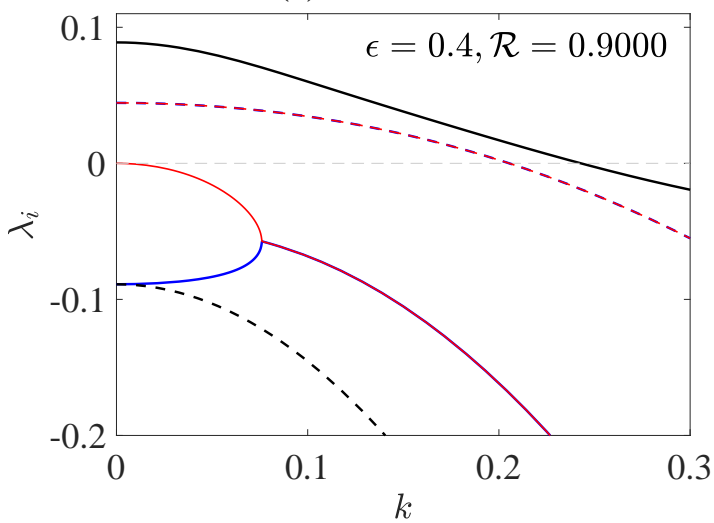

(e) $t=1.0$

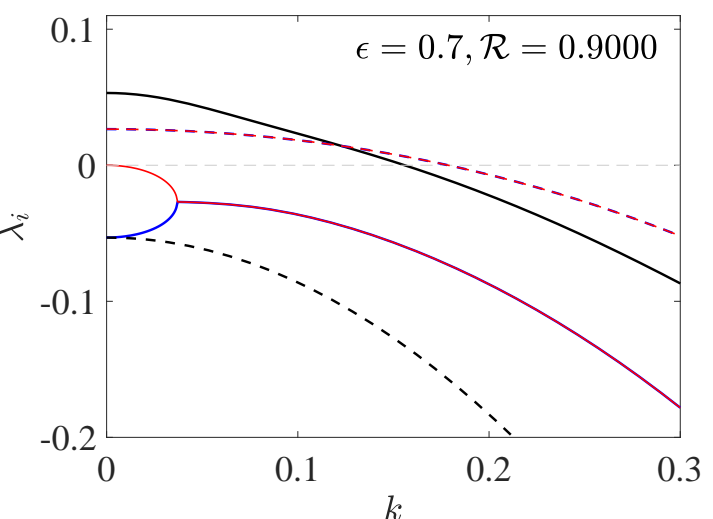

(b)

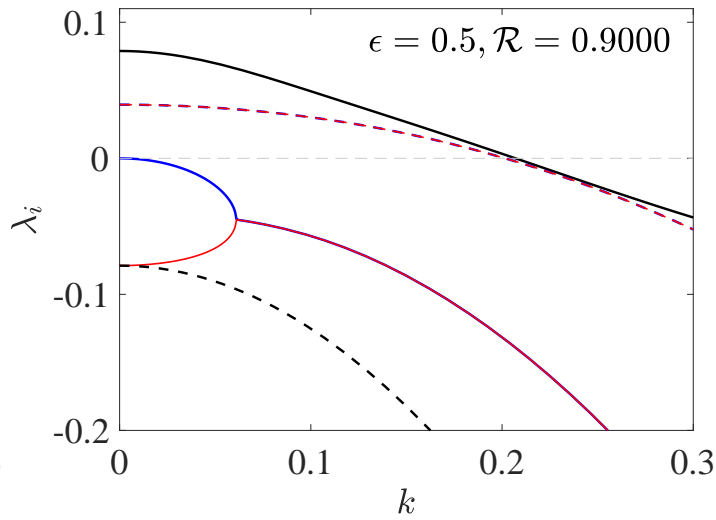

(d)

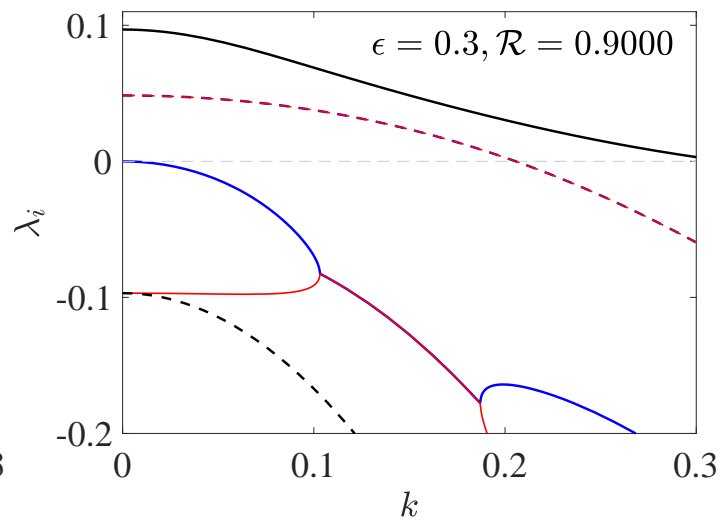

(f)

Figure 6.2: The change in the eigenvalues $\lambda_{i}(k)$ as the value of the coefficient of restitution $\epsilon$ is decreased from 0.8 (a) to 0.3 (f) at a fixed $\mathcal{R}=0.9$. With increasing dissipation, the critical value of $k$ for a charged granular gas (dashed lines) is decreased from its neutral counterpart (solid lines). 


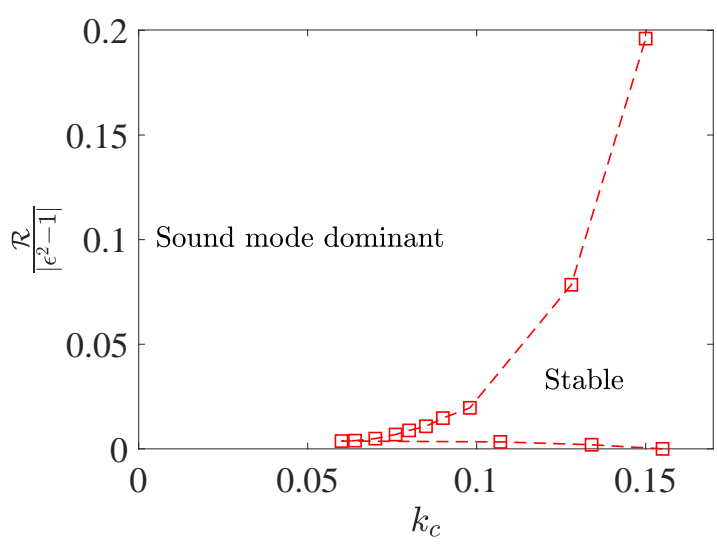

(a)

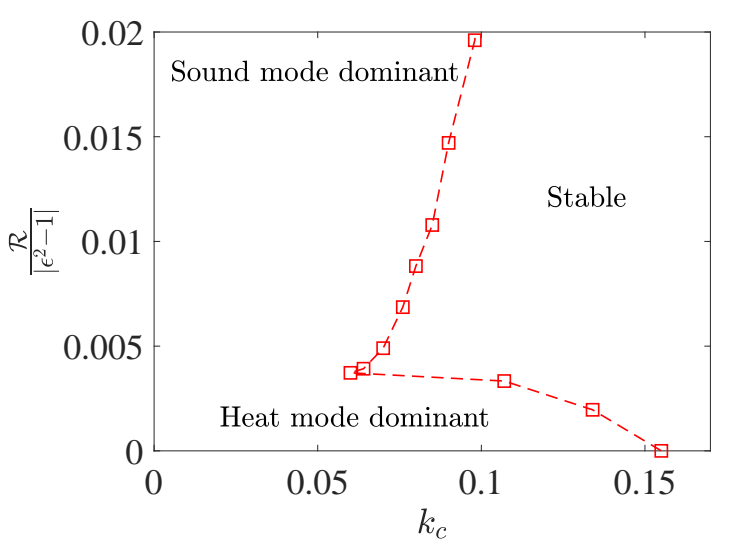

(b)

Figure 6.3: The value of the quantity $\frac{\mathcal{R}}{\left|\epsilon^{2}-1\right|}$ as a function of the critical value of $k$. The line with symbols corresponds to an unstable mode which will be excited first in the charged gas. Up to some value of $\frac{\mathcal{R}}{\left|\epsilon^{2}-1\right|}$, the heat mode will initiate the instability, while after that the sound mode will be the dominant mechanism. As $\frac{\mathcal{R}}{\left|\epsilon^{2}-1\right|}$ increases, the change from the heat mode to the sound mode is abrupt.

corresponding to unstable heat mode, it leads to the formation of (dynamic) clusters in the dissipate granular gas.

For non-zero $\mathcal{R}$ (dashed lines), the eigenvalues corresponding to heat as well as sound modes are altered. The $4 \times 4$ matrix (6.39) has rank 3, and one of the eigenvalues is zero. The non-zero eigenvalue corresponding to heat mode is now suppressed with increasing $\mathcal{R}$. The sound modes, on the other hand, tend to be unstable. At some value of $\mathcal{R}$, the two sound modes can become unstable while the heat mode is always stable. At a higher value of $\mathcal{R}$, the instability due to these two sound modes might occur even at larger $k$, or at smaller length $L$, compared to the case of a neutral granular gas [Fig. 6.1(f)].

In Fig. 6.2, the value of $\epsilon$ is varied from 0.8 to 0.3 at a fixed value of $\mathcal{R}$. As the gas becomes more dissipative, i.e. $\epsilon \rightarrow 0$, the critical value of $k$ for the electrostatic case (intersection of dashed lines at zero) becomes lower than in the neutral case (intersection of the solid line at zero). This indicates that more dissipation actually makes it harder for electrostatics to initiate the instability at smaller length scales.

The above results suggest that the mechanisms of initiation of clustering, before the longtime aggregation phase, in a charged granular gas can be distinct from the ones occurring in a neutral granular gas. The suppression of heat mode, and instability of both the sound modes, endorse this argument.

The maximum of the critical values of $k$, for which the eigenvalues intersect zero line 


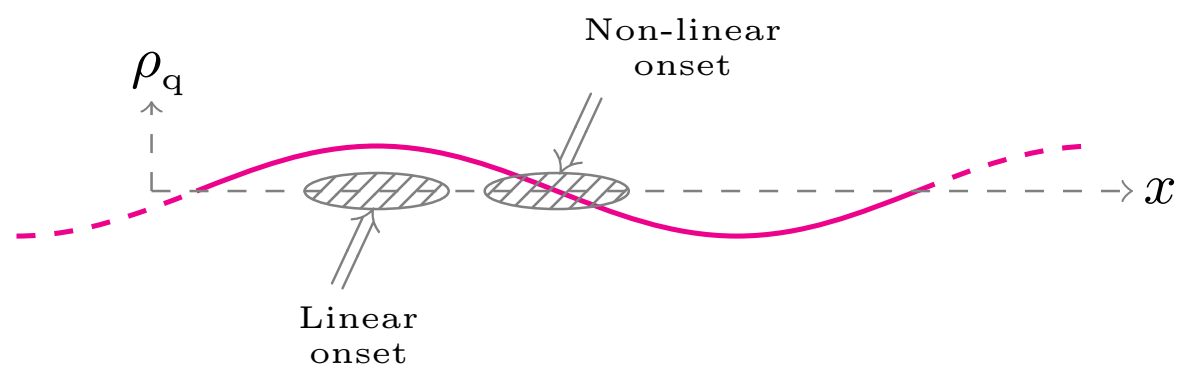

Figure 6.4: If charge is separated over some length scale, it is expected that the electrostatic origin of instability in the regions close to $\rho_{\mathrm{q}}=0$ will be non-linear in principle. In the regions which can be described with some charge density bias $\rho_{\mathrm{q} 0}$, the electrostatic origin of instability can be linear.

in Fig. 6.2, is plotted against the parameter $\frac{\mathcal{R}}{\left|\epsilon^{2}-1\right|}$ in Fig. 6.3. For small $\frac{\mathcal{R}}{\left|\epsilon^{2}-1\right|}$ the heat mode will be excited first, while as $\frac{\mathcal{R}}{\left|\epsilon^{2}-1\right|}$ increases, the sound modes become dominant.

It is important to note that in Eq. (6.27), the charge density field $\rho_{\mathrm{q}}$ is perturbed around a finite value $\rho_{\mathrm{q} 0}$. If initially a system is considered entirely neutral, i.e. with $\rho_{\mathrm{q} 0}=0$, one will find that there remain no terms which are linear in $\delta \tilde{\rho}_{\mathrm{q}}$ or $\delta \tilde{\varphi}$. This leads to the following argument. Consider a gas where the charge somehow separates over some length scale [Fig. 6.4]. On a macroscopic scale, there will be regions where $\rho_{\mathrm{q} 0}=0$ is possible. The onset of instability in these regions, if triggered by electrostatics, in principle, is expected to be non-linear. On the other hand in regions where a finite $\rho_{\mathrm{q} 0}$ is a good approximation, the onset of instability is expected to be initially linear. This is emphasized schematically in Fig. 6.4. A test case to emphasize this point is also presented in Appendix 5, where the full set of hydrodynamic equations (6.26) are evolved for given initial charge separation. 


\section{Chapter 7}

\section{Effect of induced polarization and viscous friction: Preliminary}

Charged dielectric grains are expected to polarize in their mutual electrostatic field. The resulting charge-dipole interactions $(C D)$, dipole-charge interactions $(D C)$ and dipoledipole interactions (DD) augment the monopole charge-charge interactions (CC). The reordering of the induced moments might occur at a time scale much faster than the characteristic time taken by the particles to travel their mean free path. Precise knowledge of the effect of this reordering on the aggregate growth and fractal structures is entirely lacking. These additional degrees of freedom are studied in this chapter. In addition, it is demonstrated that if the grains experience a drag force due to random interstitial "hits", the small-sized aggregates are still able to survive. 
In this chapter, two extensions of the simulations are presented:

(i) Polarized, heterogeneously charged granular gas [section 7.1], and

(ii) Polarized granular gas under viscous drag due to random interstitial velocities [section 7.2].

The preliminary results are presented in terms of cluster mass versus net dipole moment, effect on fractal dimension, and the effect on average cluster size. For the second case, the granular temperature, and the visualization of particle aggregates are also included in this chapter.

\subsection{Polarized, heterogeneously charged granular gas}

In this case, the complete set of coupled Eqs. (3.32)-(3.35) is solved. Eqs. (3.33) and (3.34) are coupled and must be solved simultaneously at each time step. In the simulations, the Jacobi iteration method is used to solve Eqs. (3.33) and (3.34). In Eq. (3.34), the Ewald summation is utilized for the computation of the $\mathrm{CC}$ interations (first term on RHS of Eq. (3.34)), while the CD interactions (second term on RHS of Eq. (3.34)) are computed up to a cutoff radius $r_{c}$. In the simulations $r_{c}=L / 2$, i.e., the standard minimum image convention. Once Eqs. (3.33) and (3.34) are solved in a coupled manner, the polarization force on a grain $i$ due to $\mathrm{CD}, \mathrm{DC}$, and DD interactions can be computed. In the next subsections, the preliminary results are presented.

\subsubsection{Cluster mass/size versus cluster dipole moment}

Figure 7.1 shows the scatter plots of the masses of aggregates, $m$, versus the magnitude of the net dipole moment of aggregates

$$
\mu \equiv\left|\sum_{i} \boldsymbol{\mu}_{i}\right| \equiv\left[\left(\sum_{i} \mu_{x, i}\right)^{2}+\left(\sum_{i} \mu_{y, i}\right)^{2}+\left(\sum_{i} \mu_{z, i}\right)^{2}\right]^{1 / 2}
$$

The index $i$ runs over all the monomers in a given aggregate. The results in Fig 7.1 indicate that larger size aggregates are expected to have relatively small magnitudes of the net dipole moment. This might be due to more homogenized dipole moments inside larger 


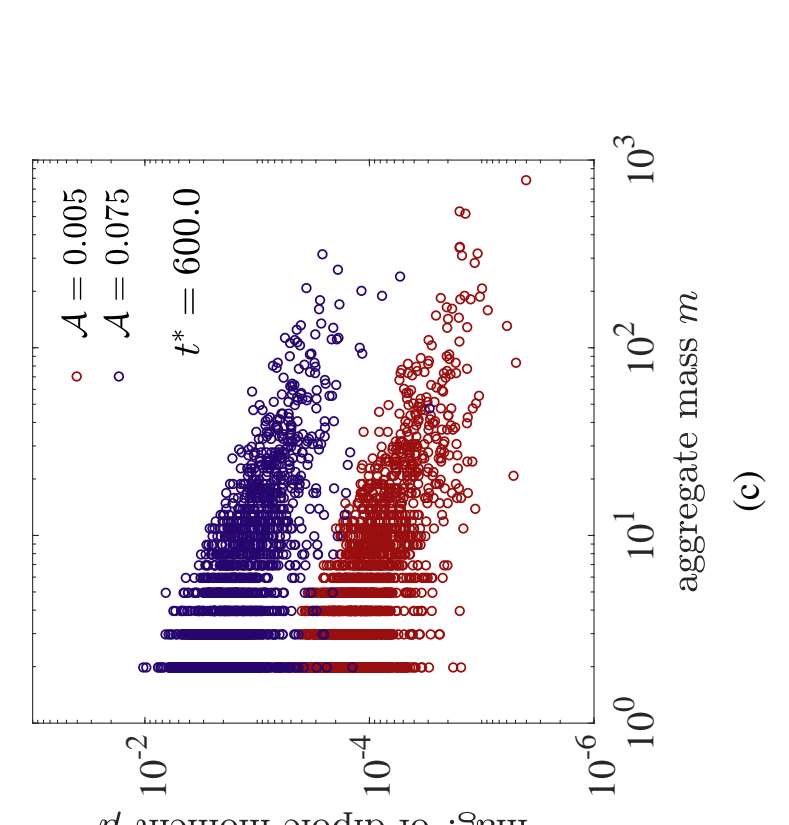

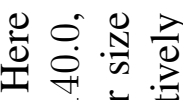

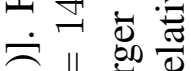

క.

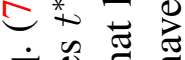

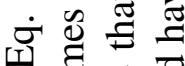

II $\Xi$ 政

귱

$\exists \cdot \frac{0}{5}$.

过

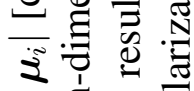

认ั

III $\stackrel{0}{0}$

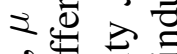

के $=:=$

के

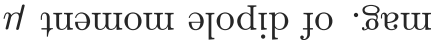

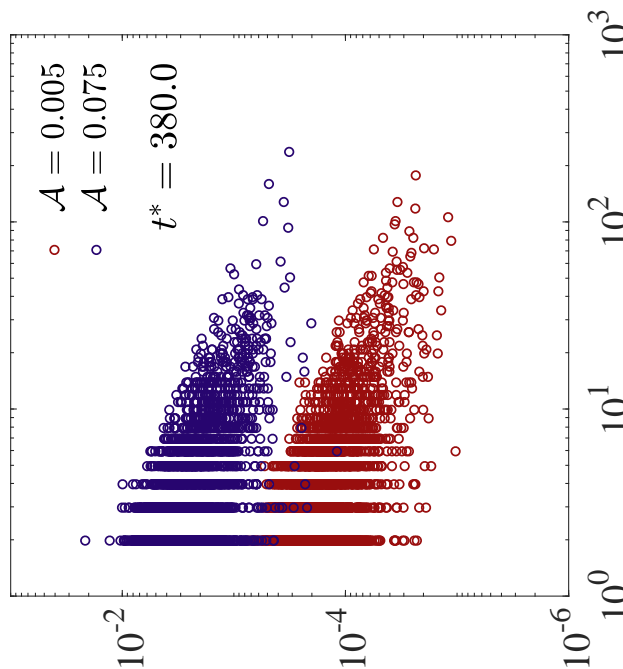

?으

논

क्ञ

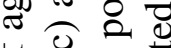

प

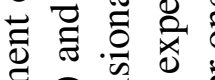

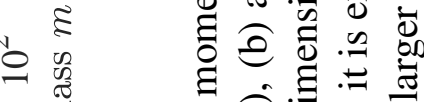

बิ

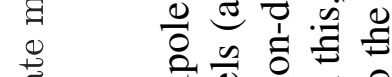

荧

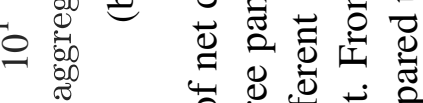

Чัญ

诃 ฮ

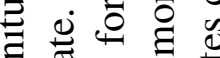

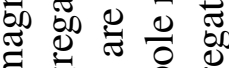

घ 5 क

\&

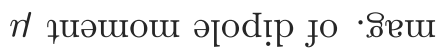

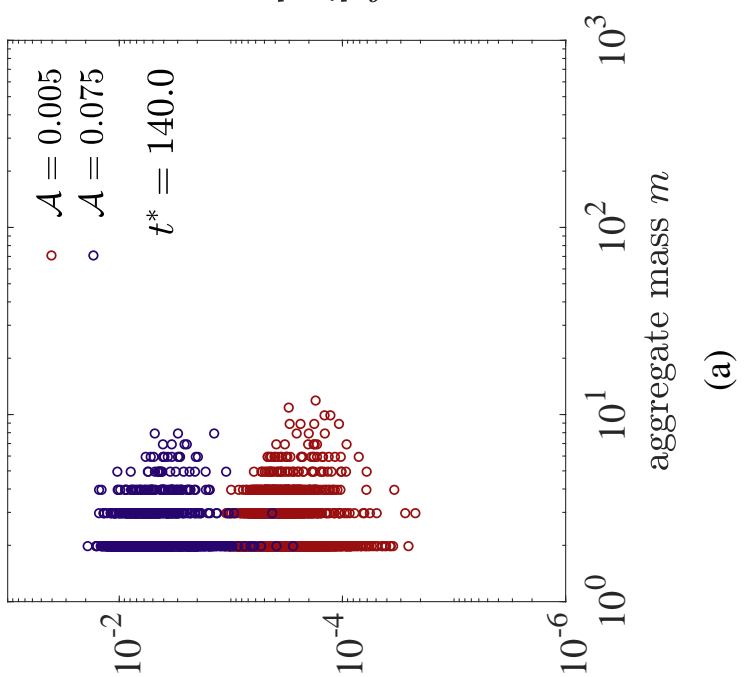

के

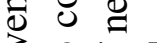

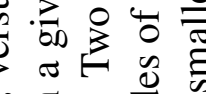

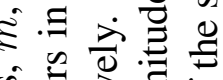

के एँ

๘్ర

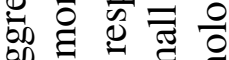

世 40 क त

응 8

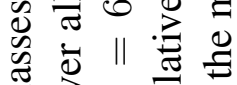

ఏ वेّ

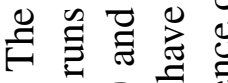

$\ddot{\sim} \sim 0$.

$\therefore$ 希

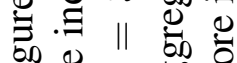

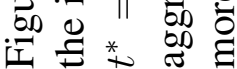




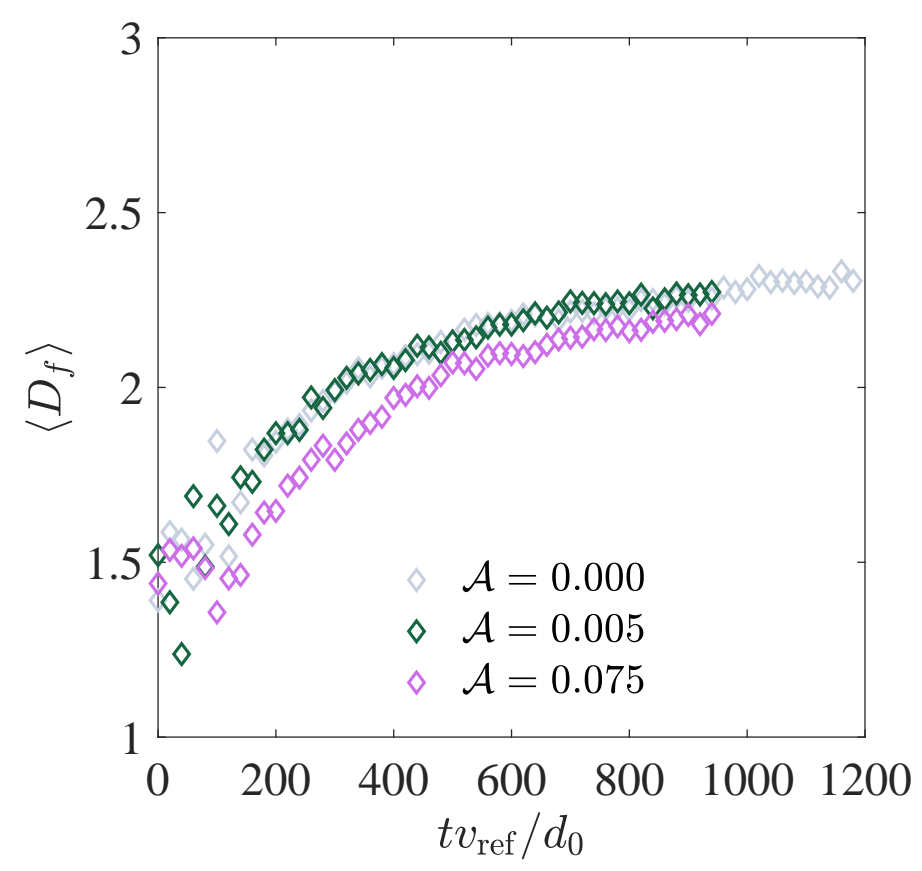

Figure 7.2: The average fractal dimension $\left\langle D_{f}\right\rangle$ with time, for different non-dimensional polarizability $\mathcal{A}$. The average fractal dimension decreases as the polarizability increases, and this effect is more pronounced at early times during the aggregation process. The initial transients during the charge-buildup $\left(t^{*} \lesssim 200\right)$ are excluded and the dynamics after charge saturation on the monomers is compared in the discussion [subsection 7.1.2].

aggregates. The smaller aggregates on the other hand, do not have enough monomers to minimize the net dipole moment. The magnitude of net dipole moment of clusters increases with with increasing polarizability. In the simulation in Fig. 7.1, the charge exchange model constructed in Eq. 4.3 is used. The other simulation parameters are $\mathcal{E}=$ 278.6, $\mathcal{D}=27.7, \mathcal{K}=1.0$. The value of $\mathcal{Q} \sim 10^{-4}=\mathcal{O}(e K)$, while a large value of the non-dimensional charge capacity $\mathcal{C}$ is set. ${ }^{1}$

In Fig. 7.1, it is also noted that the magnitude of the net dipole moment of smaller aggregates $\left(N_{\text {mon }} \leq 10\right)$ falls in a range which is almost persistent over time. As their size grows, however, the magnitude of net dipole moment is expected to decrease over time.

\subsubsection{Effect on the fractal dimension}

The simulations depict that the average fractal dimension of the aggregates may decrease due to the induced polarization. Fig. 7.2 shows the average fractal dimension with time, where three cases of non-dimensional polarizability are compared: granular gas with $(i)$

\footnotetext{
${ }^{1}$ See list of mathematical symbols and non-dimensional numbers in the front matter of the thesis, and chapter 3 for their exact definitions.
} 


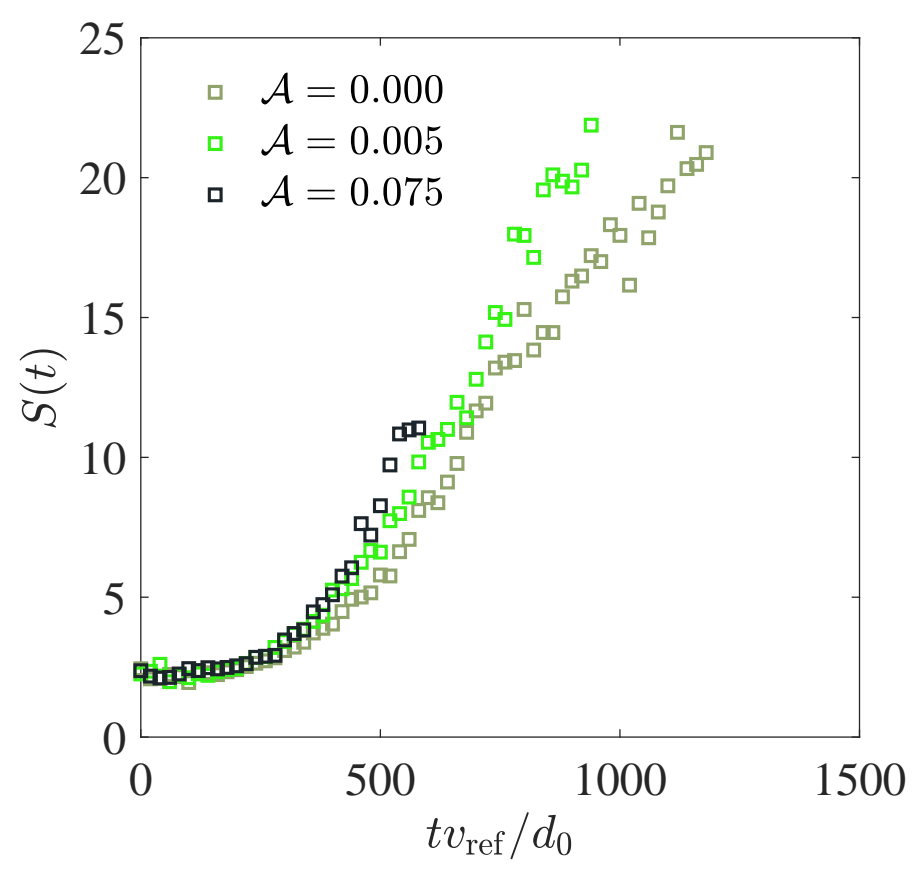

Figure 7.3: The average cluster size $S \sim\langle m\rangle^{1 /\left\langle D_{f}\right\rangle}$, with time, for different nondimensional polarizability $\mathcal{A}$. Here $\langle m\rangle=\sum_{k} m_{k}^{2} N(m) / \sum_{k} m_{k} N(m), N(m)$ being the mass distribution.

$\mathcal{A}=0.0$, (ii) $\mathcal{A}=0.005$, and (iii) $\mathcal{A}=0.075$. At very low $\mathcal{A}=0.005$, the difference is negligible. After initial transients due to charge-buildup, the increasing value of the average fractal dimension $\left\langle D_{f}\right\rangle$ signifies the compaction of the aggregates, for all the three cases. However, it is observed that at a give time after the initial transience, the value of $\left\langle D_{f}\right\rangle$ is decreased for $\mathcal{A}=0.075$. This drop in the value of $\left\langle D_{f}\right\rangle$ due to increased polarizability is also observed in the simulations if the charge holding capacity $\mathcal{C}$ is altered from a value of 1 to 10 , or higher. Also, this drop of $\left\langle D_{f}\right\rangle$ is present irrespective of whether the charge exchange is considered proportional to collision speeds [Eq. (4.3)], or it is considered stochastic and biased with the particle contact area during a collision [Eq. (3.50)]. The charge-exchange mechanisms are increasingly ineffective with decreasing granular temperature and eventually, the charge distribution has a tendency to saturate. In Fig. 7.2, the simulations parameters are same as described in subsection 7.1.1.

It is also noted that if the aggregates having monomers equal to 5 or less are excluded from the calculation of $\left\langle D_{f}\right\rangle$, there is only about $\approx 2 \%$ change in $\left\langle D_{f}\right\rangle$, while if all the aggregates are considered, the change in $\left\langle D_{f}\right\rangle$ is $\approx 8-9 \%$. This shows that the mor-

\footnotetext{
${ }^{1}$ The other simulation parameters are $\mathcal{E}=278.6, \mathcal{D}=27.7, \mathcal{K}=1.0, \mathcal{Q} \sim 10^{-4}=\mathcal{O}(e K)$, while $\mathcal{C} \gg 1$. See the list of non-dimensional number in the front matter and chapter 3 for their exact definitions.
} 


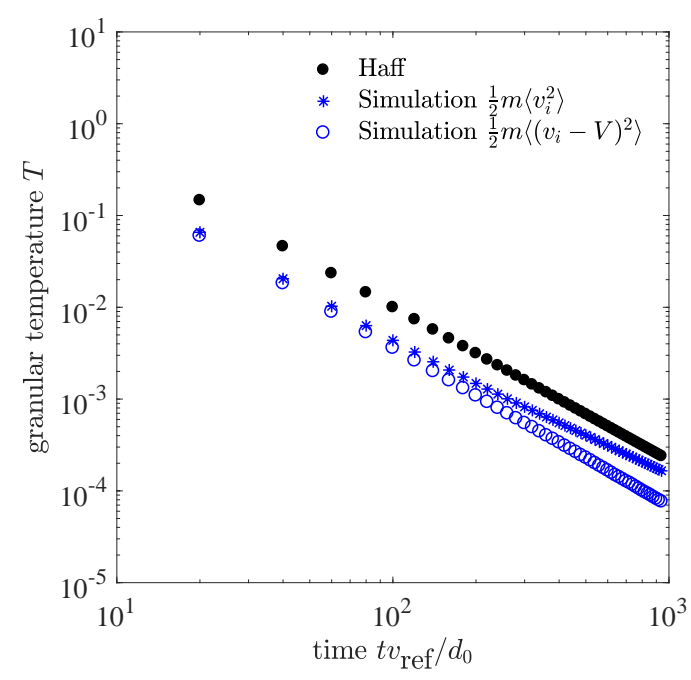

(a)

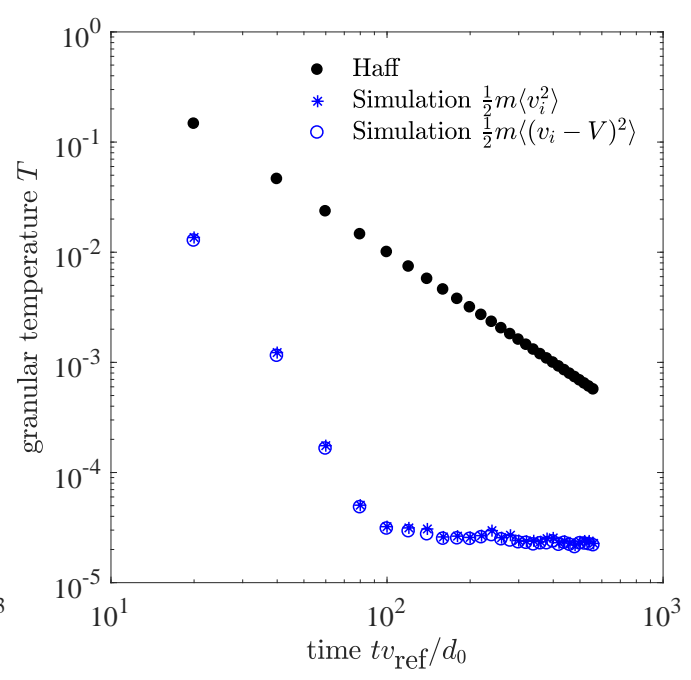

(b)

Figure 7.4: The average kinetic energy and the granular temperature of a granular gas (a) without, and (b) with the effect of viscous friction due to random interstitial velocities [see Eq. (7.2)]. Here polarizability $\mathcal{A}=0.075$ in both (a) and (b), and the Stokes number $\mathcal{S}=0.0$ in (a) while $\mathcal{S}=0.05$ in (b). In the second case, a steady state granular temperature is attained after the granular temperature reaches the order of energy input due to random interstitial fluid velocities. This case is closer to practical scenarios of dust aggregation where the influence of interstitial medium is important. In the steady state time window, the mass versus dipole moment of the fractal aggregates is studied and presented in Fig. 7.5. Here $v_{i}$ is the grain speed and $V$ is the mean velocity of grains in the vicinity of grain $i .^{1}$

phology of the smallest aggregates is more altered relative to that of the aggregates with a larger mass. It is important to note that the drop in fractal dimension due to polarization in Fig. 7.2 is more pronounced during the early stages of the growth but after the initial charge buildup ( $t^{*} \gtrsim 200$ ). The difference of fractal dimension between the cases $\mathcal{A}=0.0$ and $\mathcal{A}=0.075$ tend to decrease over time.

\subsubsection{Effect on the average cluster size}

Figure 7.3 shows the average cluster size with time for different non-dimensional polarizability. The preliminary raw data indicates an enhanced cluster growth with increasing polarizability. Notice that growth of clusters, after the initial transience, is visibly different for $\mathcal{A}=0.005$ and $\mathcal{A}=0.075$, while the evolution of $\left\langle D_{f}\right\rangle$ for these two cases is negligible in Fig. 7.2.

The data in Fig. 7.1, 7.2, and 7.3 are preliminary, and the averaging over multiple 
simulations is under progress at the time of writing of the thesis. It is expected that averaging will provide more precise growth rates while reducing the noise.

\subsection{Effect of drag due to random interstitial velocities}

To mimic the effects caused by an interstitial fluid velocity on the aggregation and fractal structures, a viscous force on the grains is applied, given by ${ }^{1}$

$$
\tilde{\mathbf{F}}_{i}^{\text {fric }}=\mathcal{S}\left(\tilde{\mathbf{v}}_{\text {fluid }}-\tilde{\mathbf{v}}_{i}\right)
$$

where $\mathcal{S}=t_{\text {ref }} / \tau_{\text {fric }}$ is the Stokes number. Here $\tau_{\text {fric }}$ is the friction time that would be taken by the particles to relax to the interstitial velocities $\tilde{\mathbf{v}}_{\text {fluid }}$, and $t_{\text {ref }}$ is some appropriately chosen reference time scale $d_{0} / v_{\text {ref. }}$. In short, as $\mathcal{S} \rightarrow 0$ the grain velocities become more and more decoupled from the fluid velocities, and vice-versa. In the simplest case presented here, the interstitial velocities $\tilde{\mathbf{v}}_{\text {fluid }}$ are random, while the grain velocities are computed from the solution of coupled Eqs. (3.32)-(3.35).

Figure 7.4 shows the average kinetic energy and the granular temperature of the granular gas of equal-sized monomers (a) without, and (b) with the effect of viscous friction due to random interstitial fluid velocities. Here polarizability $\mathcal{A}=0.075$ in both (a) and (b), and the Stokes number $\mathcal{S}=0.0$ in (a) while $\mathcal{S}=0.05$ in (b). In the second case, a steady-state granular temperature is attained after the granular temperature reaches the order of energy input due to random interstitial velocities. This case is closer to practical scenarios of dust aggregation where the influence of interstitial medium is important. In the steady-state time window, the mass versus dipole moment of the fractal aggregates is studied and presented in Fig. 7.5.

The interstitial velocities are capable of fragmenting larger aggregates formed due to long-range interactions, which is quite intuitive. However, very small aggregates (with $\leq 5$ monomers) are still able to survive [Fig. 7.5, $\mathcal{S}=0.05$ case]. Furthermore, these smallest surviving aggregates tend to form small chains [Fig. 7.6], which might be to induced polarization forces between monomers. Because the fluid velocities acting on the grains are random, there is no well-defined length scale associated with the interstitial flow

\footnotetext{
${ }^{1}$ See also Eq. (3.52) and its normalization in chapter 3.
} 
(analogous to, for example, the scale of the smallest eddies in turbulent flow). Or in other words, this setup is more like a flow where the particles are "hit" by random collisions with gas "particles" (or molecules). This setup is closer to what is described as Epstein drag regime (Capelo et al. , 2019). Such scenarios occur where the surrounding gas has very low pressure, and the Knudsen number, defined as $\mathrm{Kn}=\bar{r}_{\text {gas }} / d_{0}$, is $\gtrsim 1$. Here $\bar{r}_{\text {gas }}$ is the mean free path of the surrounding gas molecules, and $d_{0}$ is the grain diameter.

From the preliminary results in Figs. 7.5 and 7.6, it is anticipated that the gas interactions in this regime put some restraints on the continuous agglomerate growth favored by electrostatics. These local agglomerate chains might play a role of "nucleation sites" and might further influence the fluid dynamical instabilities (for example "heat mode" and "sound mode" mechanisms studied in chapter 6). As one relevant example, fluid dynamical mechanisms of density variations have been explored recently in experimental work by Capelo et al. (2019) for conducting grains. The researchers found the formation of small scale dynamic clusters in the presence of a laminar incompressible gas stream.

\subsection{Conclusions}

The preliminary results presented in this chapter have provided a strong indication that the growth of average cluster size is enhanced [Fig. 7.3] while the fractal dimension is lowered [Fig. 7.2]. The smaller aggregates in the cluster population are relatively more affected due to these degrees. As the size of aggregates grows, the magnitude of the net dipole moment on them decreases [Fig. 7.1]. Results from the implementation of friction due to random interstitial velocities have shown that a steady-state aggregation-fragmentation regime can be reached [Fig. 7.4], and only small-sized aggregates are expected to survive [Fig. 7.5]. Furthermore, these smallest surviving aggregates, due to induced polarization forces between monomers, form very small chains [Fig. 7.6], consisting of $\approx 2$ to 6 monomers. These local agglomerate chains might play a role of "nucleation sites" and might further influence the fluid dynamical instabilities (for example "heat mode" and "sound mode" mechanisms studied in chapter 6).

From these outcomes, few general remarks can be made in the context of clustercluster aggregation (CCA) models, where two clusters collide and make a larger cluster. Corresponding to the gas simulated in this chapter, the interactions in the CCA model 
can be broken into cases: between small-small, small-large, and large-large clusters. It can be considered that the interactions between large-large clusters can be simplified to only CC level, while interactions between small-small or small-large clusters need to be augmented by CD, DC and DD forces. ${ }^{1}$

Furthermore, the polarization and the mechanism involving reordering of the dipole moments might even cause aggregation in a gas of like-charged particles (i.e., where all the particles carry same sign of charge). This has been rarely shown theoretically (except, for example, by Matthews \& Hyde (2009) for charged dust-aggregation in plasma environment). As shown in previous chapter 4 [Fig. 4.1(a)], a gas of monopoles with like-charges is simply a repulsive gas where collision frequency keeps decreasing over time and no aggregation appears. However, to study like-charged gas under polarization forces is a future scope and may show some qualitative differences from a gas of only like-charged monopoles.

${ }^{1}$ The size distribution in the aggregating granular gas is more like a continuous one [Fig. 4.4, chapter 4]. The scenario of interactions between small-small, small-large and large-large clusters should be considered/pictured accordingly. 


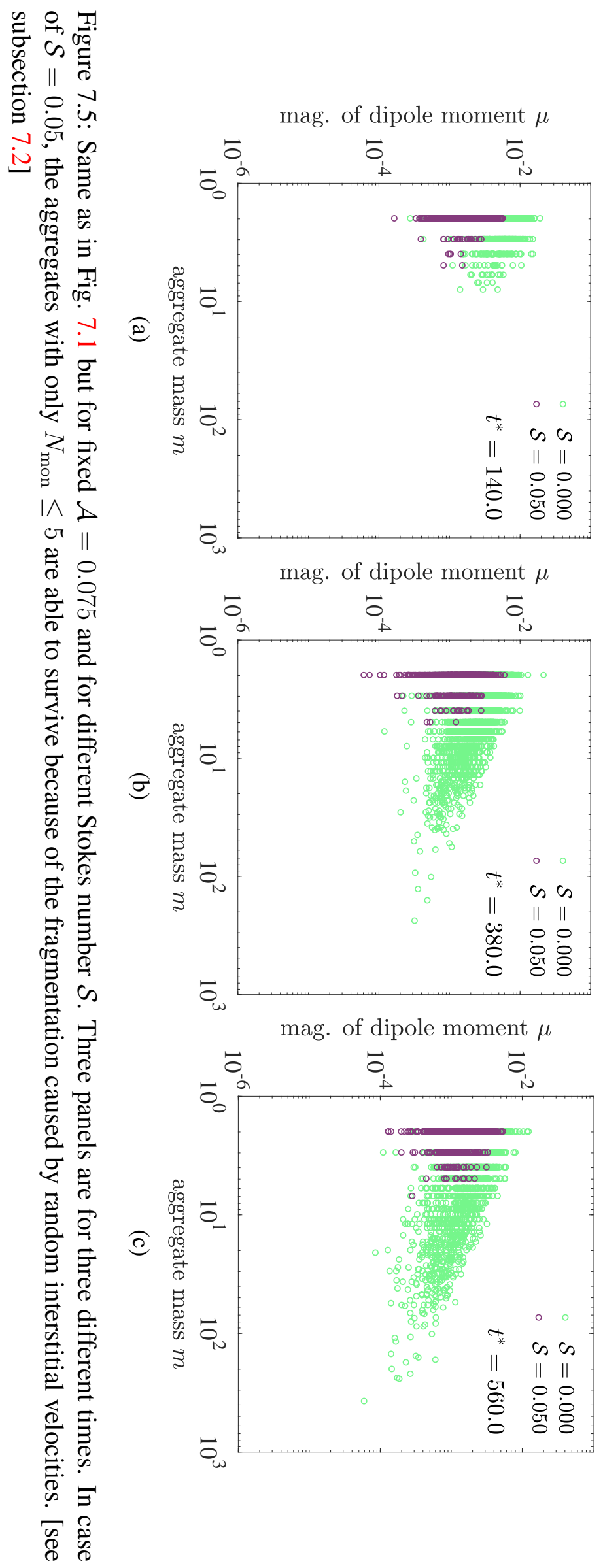




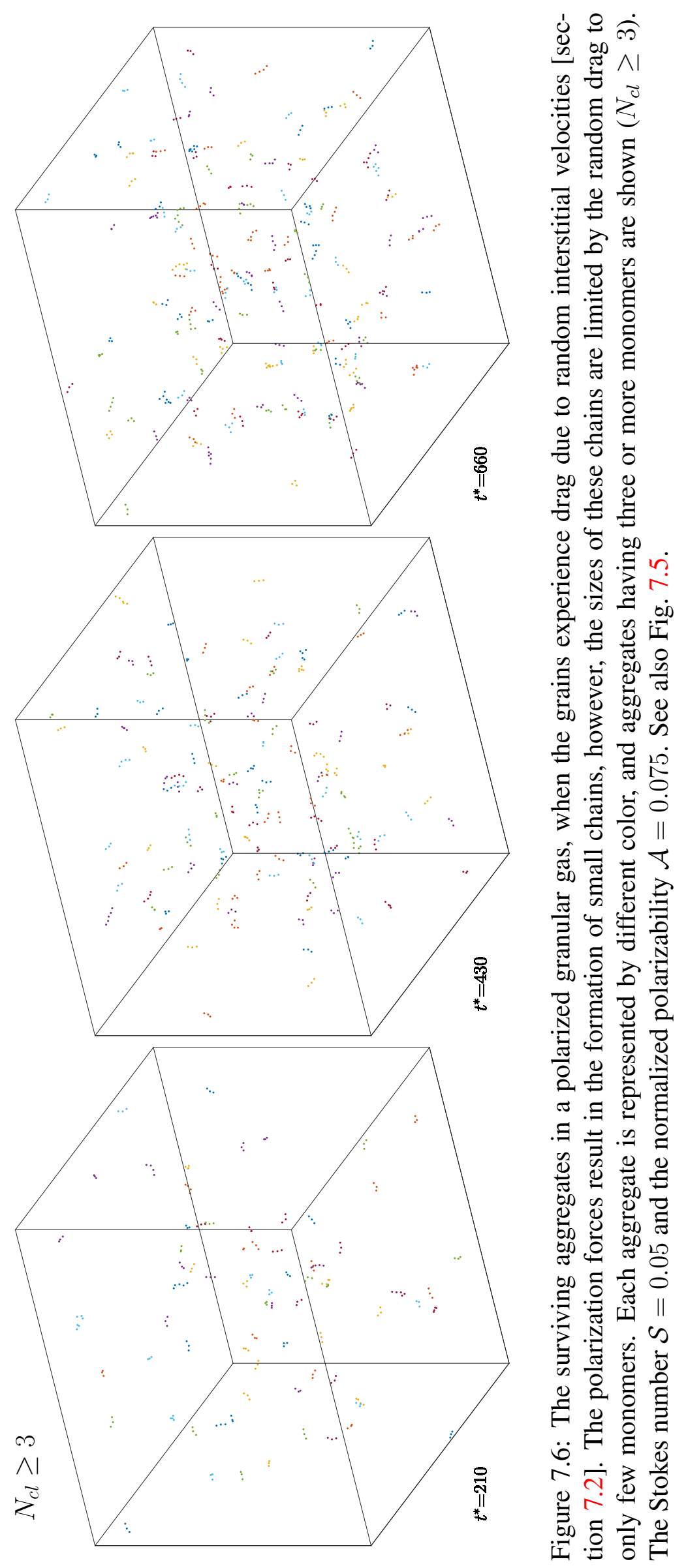





\section{Chapter 8}

\section{Conclusions, Outlook, and Extensions}

Imagine a collection of agitated dielectric grains in which (notoriously) a scalar quantity gets transported across the grains on completely different (and unknown) time scales than the time scales associated with the motion of the grains themselves. This notorious quantity, furthermore, may "jump" or "travel" from places to places on the surface of the grains in a mysterious manner. Why we should try to catch it and why not let it do whatever it wants to do? The problem is that it causes "interruptions" to the independent motion of the grains. Because the "travel" of this quantity is mysterious, the "interruptions" caused by it become hard to predict. The quantity interconnects the motion of all the grains on which it decides to live. When everything seems to settle down, it "reorders" itself on the grain surfaces because it sees that its "friends" on the other grains are doing the same.

The quantity is the electric charge, while some examples of "interruptions" are clogging in pipes and hoppers during industrial conveying of grains, increase in saltation thresholds of wind-blown sand beds, coagulation of planetary dust, and so on. In the above paragraph, "reordering" implies the induced polarization effect.

Although the hard condensed matter principles of contact or frictional electrification remain elusive, here an effort is made to theoretically understand the collective/manybody effects in dissipative granular gases, once the grains are charged. Specifically, the aggregation and pattern formation in heterogeneously charged, globally charge conserving, and initially dilute granular gases is focused; the gas is charged with simplified but physically valid charge-exchange recipes and the subsequent effects of the long-range forces on the dynamics and morphology are studied. Canonical observables, such as 
growth rates of the average cluster size, average fractal dimension, granular temperature, and charge variance are computed using granular molecular dynamics simulations. In addition, the size, velocity and charge distributions are also studied. The observations from the detailed numerical simulations are utilized to (i) modify the mean-field Smoluchowski's coagulation equation, and (ii) to provide a kinetic description taking care of restitution as well as aggregation. The onset of clustering instability and the competition between dissipation and electrostatics, on a macroscopic scale, is then studied via the linear stability analysis of the granular hydrodynamic equations. Finally, the alterations in the aggregate growth rates and the average fractal dimension due to additional degrees of freedom, introduced by the grain polarizability, are briefed.

\subsection{Summary of conclusions}

The overall conclusions from the thesis are summarized below.

The presence of charges in granular gases leads to aggregate formation, rather than dynamic clustering [Figs. 4.2, 4.3, 5.1]. Here, by dynamic clustering, it is meant that the density variations emerge due to dissipation, but no literal sticking of grains appears. The dynamic clustering, however, might also be present in the charged granular gas at mesoscopic scales where individual aggregates behave as "newborn" particles [see Fig. 5.7(c) inset for Mach number, and movies accompanying the thesis]. The crossover time of the onset of aggregation reduces with increasing ratio of the characteristic Coulomb to kinetic energy $\mathcal{K}$ [Fig. 4.5]. When compared on a coarse-grained scale, the average cluster size increases faster in a charged granular gas than a neutral granular gas [Fig. 4.5]. The faster growth of aggregates in a charged granular gas is explained by suppression of diffusion of particles in their mutual electrostatic field. This observation of suppressed MSD from the simulations, when incorporated into the reaction kernel of the Smoluchowski's coagulation equation, also supports an enhanced growth rate [Fig 4.6]. The simulations have indicated charge-velocity correlations in the gas [Fig. 4.8]. The high-velocity monomers have shown to carry relatively low magnitudes of charge; the low-velocity monomers may or may not. These high-velocity particles create non-relaxing stretched tails in the velocity distribution function of the monomers [Fig. 4.7], and imply that the granular gas is fetched further from equilibrium than its neutral counterpart (at least during the transience if there 
exist an eventual equilibrium state).

The aggregates in the charged granular gas are statistically self-similar (fractals), and this feature remains persistent during the course of aggregation. The masses of aggregates have a power-law relation with their sizes, characterized by the average fractal dimension [Fig. 5.5]. The value of the average fractal dimension indicates compaction of the aggregates with time; with time its value is increased from the typical values for ballistic cluster-cluster aggregation $(\mathrm{BCCA})(\sim 1.8-2.0)$ to the typical values for diffusion limited particle-cluster aggregation (DLPCA) $(\sim 2.5)$.

Once the aggregation sets in, the aggregates might be thought of as "newborn" particles. The average quantities such as number density, temperature, charge variance etc. of these "newborn" particles are computed [Figs. 5.3, 5.7]. Up to the simulated times, the granular temperature of the gas of "newborn" particles remains close to the modified Haff's law for viscoelastic granular gas of monomers. The charge variance drops during aggregation, provided that the charge on individual monomers is saturated [Fig. 5.6]. The dynamics of number density [Fig. 5.7(c)], and the size distribution [Fig. 4.4] of these "newborn" particles are more involved; the number density evolution is non-monotonic and the size distribution broadens over time as the aggregation proceeds.

One important result is that once the aggregates are formed, the granular temperature of cluster population $T(t)$, the charge variance of cluster population $\left\langle\delta q^{2}\right\rangle(t)$ and the typical aggregate size in the cluster population $d(t)$, evolve in such a way that their non-dimensional combination

$$
\mathcal{B}(t)=\frac{k_{e}\left\langle\delta q^{2}\right\rangle(t)}{T(t) d(t)}
$$

obeys a physical constraint of $\mathcal{B}(t) \leq 1$. This constraint on the collective evolution of charged clusters is confirmed both by the developed kinetic model [Eq. (5.6)-(5.8)] and the detailed molecular dynamics simulations [Figs. 5.3, 5.4]. In the above equation, $k_{e}$ is the Coulomb constant. It is also showed that the application of a purely dissipative kinetic treatment is not sufficient to make predictions about global observables such as $T$ and $\left\langle\delta q^{2}\right\rangle$ in an aggregating charged granular gas [Fig 5.4], and an appropriate effective coefficient of restitution needs to be augmented in the kinetic-theory [Fig 5.2].

The onset of instability is also explored via the linear stability analysis of the hydro- 
dynamic equations for a granular gas coupled to Maxwell's electrostatic equations, in a certain regime of the granular temperature [chapter 6]. In this limit, the transport coefficients are expected to remain close to that of neutral granular gas. The presence of charge alters the so called "heat mode" and "sound modes" of instability [Figs. 6.1, 6.2, 6.3]. It is found that electrostatics is expected to favor "sound modes", rather than the "heat mode", at low granular temperatures. The difference between the two is that in the "sound modes", the onset of instability is triggered by the growth of temperature and velocity perturbations, while in the "heat mode" it is triggered by the density variations. Thus, it implies that electrostatics is expected to favor velocity and temperature variations first, and then the density variations emerge.

The preliminary results presented in chapter 7 have provided a strong indication that because of the induced polarization, the growth of average cluster size is enhanced [Fig. 7.3] while the fractal dimension is lowered [Fig. 7.2]. The smaller aggregates in the cluster population are relatively more affected due to these degrees. As the size of aggregates grows, the magnitude of the net dipole moment on them decreases [Fig. 7.1]. Results from the implementation of friction due to random interstitial velocities have shown that a steady-state aggregation-fragmentation regime can be reached [Fig. 7.4], and only smallsized aggregates are expected to survive [Fig. 7.5]. Furthermore, these smallest surviving aggregates, due to induced polarization forces between monomers, form very small chains [Fig. 7.6], consisting of $\approx 3$ to 6 monomers. These local agglomerate chains might play a role of "nucleation sites" and might further influence the fluid dynamical instabilities (for example "heat mode" and "sound mode" mechanisms studied in chapter 6).

From these outcomes in chapter 7 , few general remarks can be made in the context of cluster-cluster aggregation (CCA) models, where two clusters collide and make a larger cluster. The interactions in the CCA model can be broken into cases: between small-small, small-large, and large-large clusters. It can be considered that the interactions between large-large clusters can be simplified to only CC level, while interactions between smallsmall or small-large clusters need to be augmented by CD, DC and DD forces. ${ }^{1}$

Furthermore, the polarization and the mechanism involving reordering of the dipole

\footnotetext{
${ }^{1}$ The size distribution in the aggregating granular gas is more like a continuous one [Fig. 4.4, chapter 4]. The scenario of interactions between small-small, small-large and large-large clusters should be considered/pictured accordingly.
} 


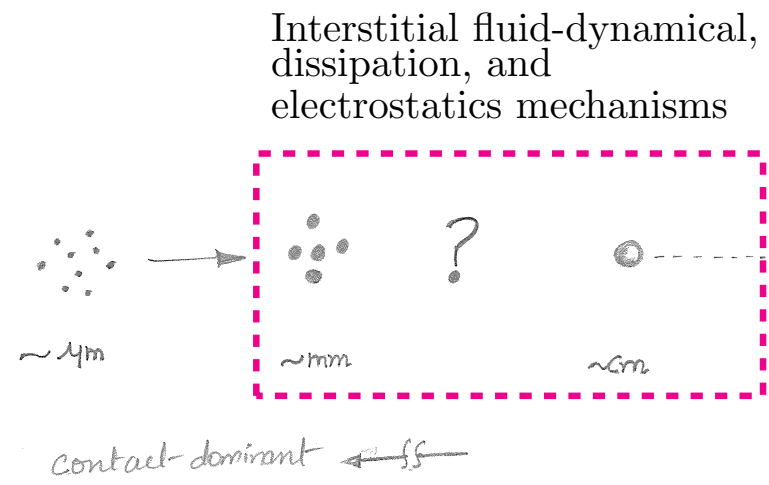

e.g. Van der Waals

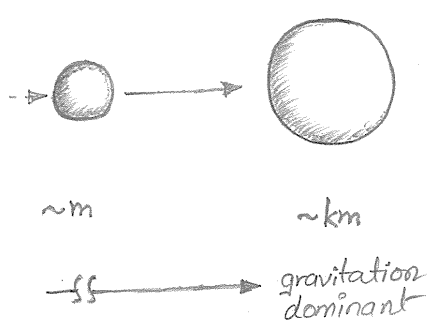

Collapse

Figure 8.1: Different grain size regimes and corresponding mechanisms of growth. The results from this thesis support the following school of thought (Spahn \& Seiß, 2015): in the grain size regime $d_{0} \sim \mathrm{mm}-\mathrm{cm}$, electrification and polarization of grains might be a dominant mechanism, which is capable of producing aggregate sizes sufficient for subsequent gravitational collapse $\left(d_{0} \gtrsim \mathrm{m}\right)$. For grain sizes $d_{0} \lesssim \mu \mathrm{m}$, cohesive forces such as Van der Waals are important in the growth process.

moments might even cause aggregation in a gas of like-charged particles (i.e., where all the particles carry same sign of charge). This has been rarely shown theoretically (except, for example, by Matthews \& Hyde (2009) for charged dust-aggregation in plasma environment). As shown in previous chapter 4 [Fig. 4.1(a)], a gas of monopoles with like-charges is simply a repulsive gas where collision frequency keeps decreasing over time and no aggregation appears. However, to study like-charged gas under polarization forces is a future scope and may show some qualitative differences from a gas of only like-charged monopoles.

\subsection{Outlook and possible extensions}

The theoretical description of pattern formation in dissipative, charged and polarized particulate systems is a formidable task. Especially for practical scenarios, the aggregation dynamics needs to be augmented with an accurate description of the (turbulent) gaseous flow fields around them. The unresolved hard condensed matter principles of contact or triboelectric charging add to the complexity. In particle-laden flows, often the collision mechanics and dissipation during collisions is simplified. Thus, a rigorous four-way coupled model for polarized particles with more accurate modeling of the triboelectric 
charging, and resolution of the charge dynamics on the grain surfaces are tasks left to the future. The dynamics of charge on the gain surface is expected to be occurring at a different time scale than the time scales of a collision, or the time scales of the mean free path. The interstitial flow would introduce its own time scale. Thus in principle, the problem is multi-scale and at this moment lacks rigorous understanding. Another complexity is the computational expense introduced by the long-range nature of electrostatics. Even the development of accurate time-saving mean-field models for this problem would require at least some knowledge from either careful experiments or simulations taking care of all or most of the above degrees of freedom. Precise inclusion of the above mechanisms might also resolve the "meter barrier" problem in planetesimal formation [Fig. 8.1]. Intrinsic presence of the charge-velocity correlations and aggregation (gas is no more dilute locally) makes Boltzmann-like descriptions questionable later in time or at least requires further development for the long-time solutions. 


\section{Appendix 1: Accompanying material}

The following media and codes accompany this thesis:

(i) A movie of the evolving and aggregating structures in the charged granular gas (in a compact disk attached with the thesis).

(ii) An in-house cluster analysis code in MATLAB to obtain the fractal dimension, cluster size distribution, average cluster size, and other statistical quantities, which is made open-source at https://gitlab.com/cphyme/matbox.

(iii) An in-house MATLAB program to solve coupled hydrodynamic equations for homogeneous quasi-monodisperse aggregation. This program is also a part in the open-source code https://gitlab.com/cphyme/matbox. 



\section{Appendix 2: Overall algorithm}

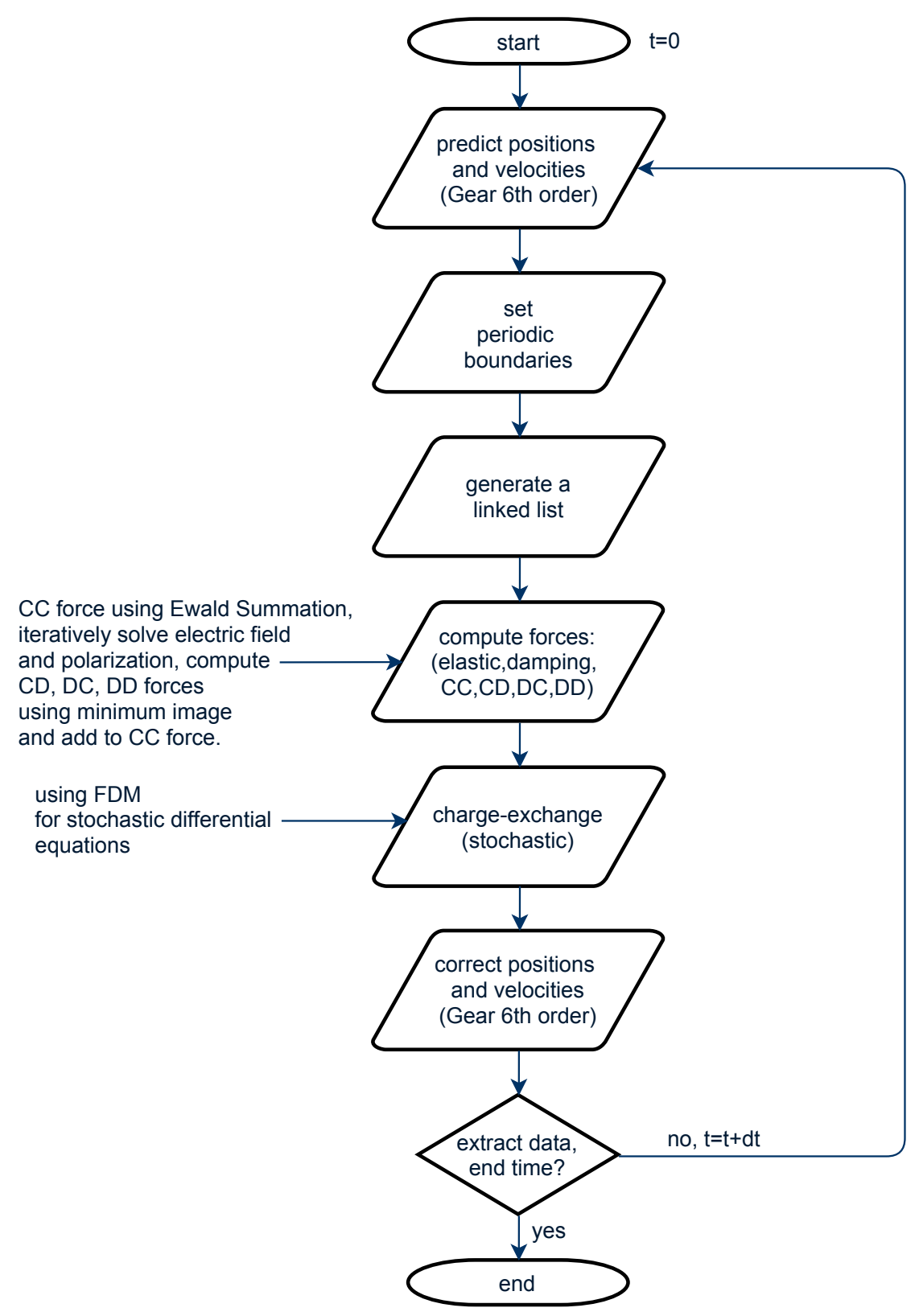

Figure A2.2: Overall granular molecular dynamics algorithm, and incorporation of charge-exchange and induced polarization 



\section{Appendix 3: MATLAB Scripts}

\section{Script for eigenvalues of the linear stability matrix}

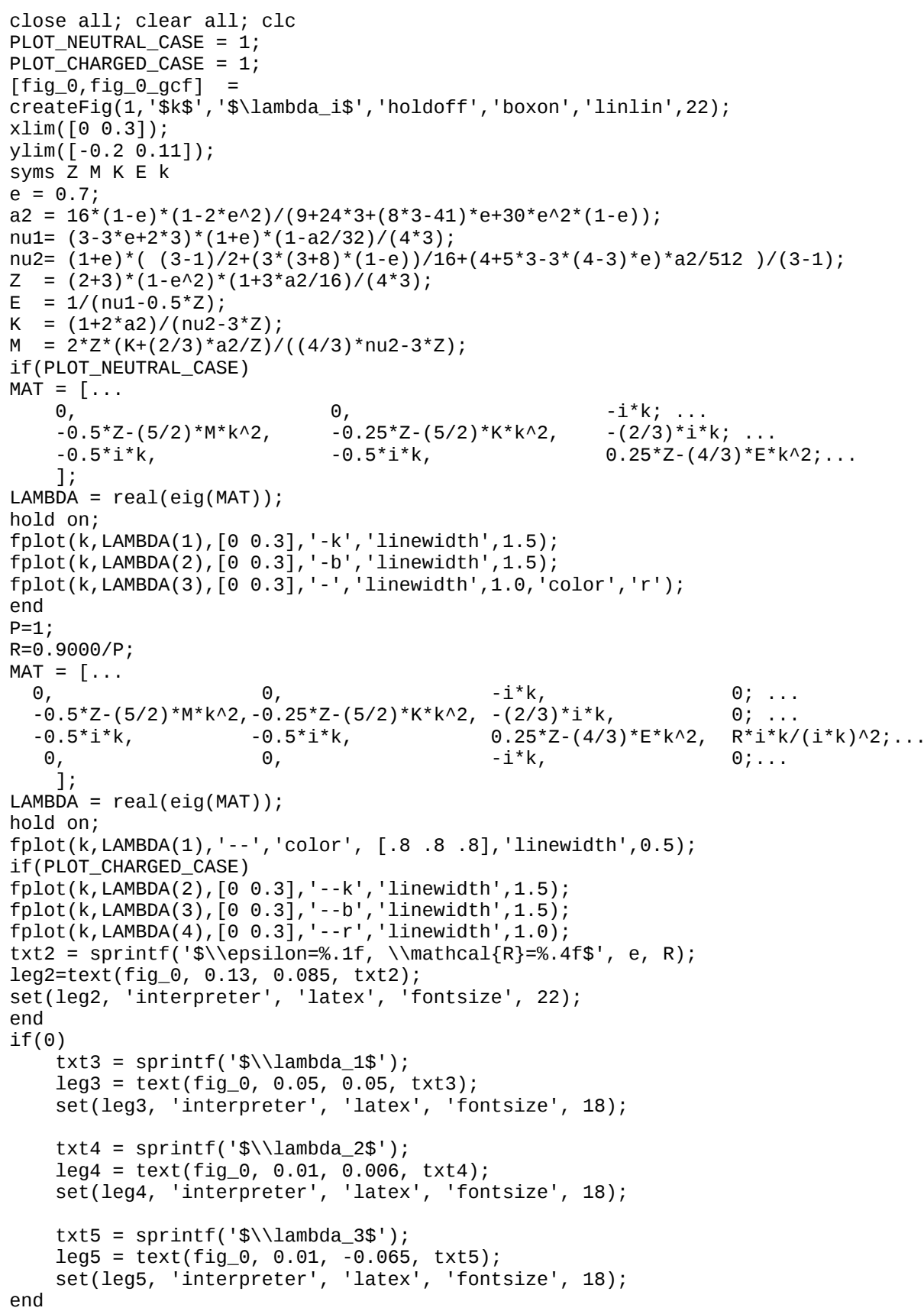




\section{Approximation of the scaled cooling and transport coeffi- cients for a gas with $\epsilon=$ const., in the limit $\frac{1}{\left|\epsilon^{2}-1\right|} \cdot \frac{q_{\mathrm{ref}}^{2} k_{e}}{\bar{r}} \ll T$}

The following set of scaled transport coefficients is used in the linear stability analysis in Chapter 6 (Brilliantov \& Pöschel, 2010):

$$
\begin{aligned}
\eta^{*} & =\frac{1}{\nu_{1}^{*}-\frac{1}{2} \zeta^{*}}, \\
\kappa^{*} & =\frac{1+2 a_{2}}{\nu_{2}^{*}-3 \zeta^{*}}, \\
\mu^{*} & =2 \zeta^{*} \frac{\kappa^{*}+\frac{2 a_{2}}{3 \zeta^{*}}}{\frac{4}{3} \nu_{2}^{*}-3 \zeta^{*}}, \\
\zeta^{*} & =\frac{5}{12}\left(1-\epsilon^{2}\right)\left(1+\frac{3 a_{2}}{16}\right),
\end{aligned}
$$

where

$$
\begin{aligned}
& \nu_{1}^{*}=\frac{(9-3 \epsilon)(1+\epsilon)}{12}\left(1-\frac{a_{2}}{32}\right), \\
& \nu_{2}^{*}=\frac{1+\epsilon}{2}\left(1+\frac{33(1-\epsilon)}{16}+\frac{19-3 \epsilon}{512} a_{2}\right), \\
& a_{2}=\frac{16(1-\epsilon)\left(1-2 \epsilon^{2}\right)}{81-17 \epsilon+30 \epsilon^{2}(1-\epsilon)} .
\end{aligned}
$$

Notice that for $\epsilon=1$ (elastic gas), $a_{2}=0, \nu_{1}^{*}=1$ and $\nu_{2}^{*}=1$. The scaled transport coefficients in this case are constants.

\section{Moving from Ewald potential to forces}

The following simple symbolic script can be run in MATLAB to differentiate the Ewald potential in Eq. (3.19):

clear all;
close all;

clc;

syms b qi qk ke pi L m rik ri rk

Uri real $=k e^{*} q k^{*} \operatorname{erfc}\left(b^{*}\right.$ abs $\left.(r k-r i)\right) / a b s(r k-r i)$

$\mathrm{Fi}$ real $=-$ qi*gradient (Uri real, ri)

Urí_reciprocal $=\left(\mathrm{ke}^{*} 4 * \mathrm{pi} / \mathrm{L}^{\wedge} 3\right) * \mathrm{qk} *(1 / \mathrm{abs}(\mathrm{m}) \wedge 2) * \exp \left(-\operatorname{abs}(\mathrm{m}) \wedge 2 /\left(4 * \mathrm{~b}^{\wedge} 2\right)\right) * \cos (\mathrm{m} *(\mathrm{rk}-\mathrm{ri}))$

$\mathrm{Fi}$ reciprocal $=-$ qi*gradient $($ Uri_reciprocal, $\mathrm{ri})$ 


\section{Appendix 4: MATHEMATICA Scripts}

\section{Dipole-dipole interaction (DD) added to the MD code}

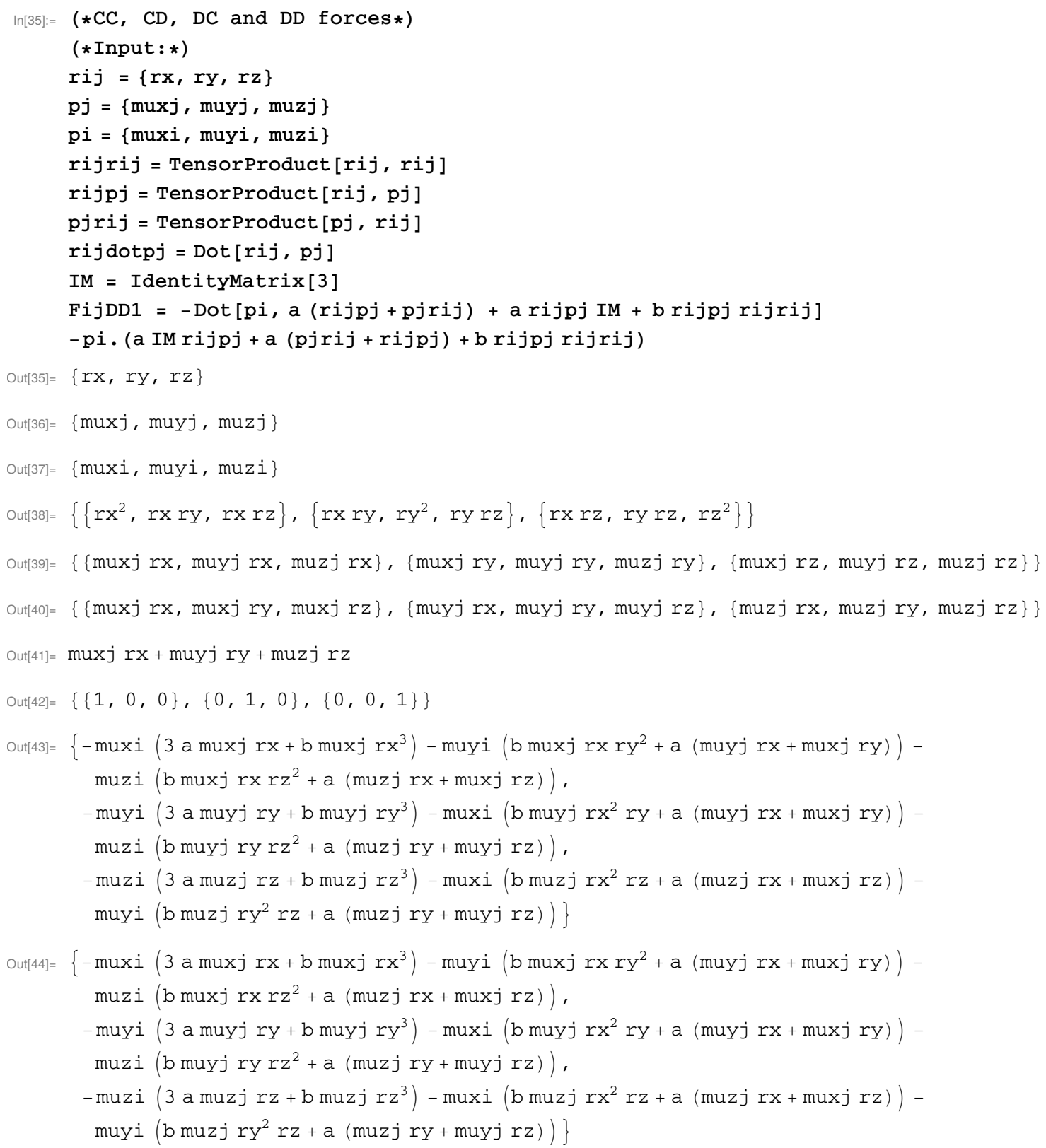




\title{
MATHEMATICA instructions to solve kinetic integrals
}

\author{
(*These are the MATHEMATICA instructions to solve the restitutive, \\ aggregative, and some other useful integrals. Some of the integrals below are \\ expensive and might take some time in MATHEMATICA. The last instruction \\ at the bottom of this script can be used to clear all the results, \\ so that the script can be run again.*) \\ Integrate $\left[\mathrm{x}^{\wedge} 2 /\left(\mathrm{E}^{\wedge}(\mathrm{b} \mathrm{x} \mathrm{Y}) \mathrm{E}^{\wedge} \mathrm{x}^{\wedge} 2 \mathrm{E}^{\wedge} \mathrm{Y}^{\wedge} 2\right),\{\mathrm{y}, 0\right.$, Infinity $\},\{x, 0$, Infinity $\left.\}\right]$ \\ Integrate $\left[\mathrm{x}^{\wedge} 2 /\left(\mathrm{E} \wedge(\mathrm{b} \mathrm{x} \mathrm{y}) \mathrm{E}^{\wedge} \mathrm{x} \wedge 2 \mathrm{E}^{\wedge} \mathrm{y}^{\wedge} 2\right),\{\mathrm{y},-\right.$ Infinity, 0$\},\{x,-$ Infinity, 0$\left.\}\right]$ \\ Integrate $\left[\mathrm{x}^{\wedge} 2 /\left(\mathrm{E} \wedge(\mathrm{bx} \mathrm{y}) \mathrm{E}^{\wedge} \mathrm{x} \wedge 2 \mathrm{E} \wedge \mathrm{y}^{\wedge} 2\right),\{\mathrm{y}, 0, \operatorname{Infinity}\},\{\mathrm{x}, 0, \operatorname{Infinity}\}\right]+$ \\ Integrate $\left[\mathrm{x}^{\wedge} 2 /\left(\mathrm{E}^{\wedge}(\mathrm{b} \mathrm{XY}) \mathrm{E}^{\wedge} \mathrm{x}^{\wedge} 2 \mathrm{E}^{\wedge} \mathrm{Y}^{\wedge} 2\right),\{\mathrm{y},-\right.$ Infinity, 0$\},\{x,-$ Infinity, 0$\left.\}\right]$ \\ Integrate $\left[\mathrm{E}^{\wedge}\left(-\mathrm{x}^{\wedge} 2-\mathrm{y}^{\wedge} 2\right) \mathrm{x}^{\wedge} 2(1-\mathrm{bxy}),\{\mathrm{y}, 0, \operatorname{Infinity}\},\{\mathrm{x},-\operatorname{Infinity}, 0\}\right]$ \\ Integrate $\left[\mathrm{E}^{\wedge}\left(-\mathrm{x}^{\wedge} 2-\mathrm{y}^{\wedge} 2\right) \mathrm{x}^{\wedge} 2(1-\mathrm{bxy}),\{\mathrm{y}, 0\right.$, Infinity $\},\{x,-$ Infinity, 0$\left.\}\right]+$ \\ Integrate $\left[E^{\wedge}\left(-x^{\wedge} 2-y^{\wedge} 2\right) x^{\wedge} 2(1-b x y),\{y,-\right.$ Infinity, 0$\},\{x, 0$, Infinity $\}$ \\ Integrate[r(2+Abs[b] $r \wedge 2 \operatorname{Sin}[t] \operatorname{Cos}[t]) E \wedge(-A b s[b] r \wedge 2 \operatorname{Sin}[t] \operatorname{Cos}[t]) E \wedge(-r \wedge 2)$, \\ $\{r, 0, \operatorname{Infinity}\},\{t, 0, P i / 2\}]+$ \\ Integrate[r(2+Abs[b] $r \wedge 2 \operatorname{Sin}[t] \operatorname{Cos}[t]) E \wedge(-A b s[b] r \wedge 2 \operatorname{Sin}[t] \operatorname{Cos}[t]) E \wedge(-r \wedge 2)$, \\ $\{r, 0$, Infinity $\},\{t, P i, 3 \mathrm{Pi} / 2\}]$

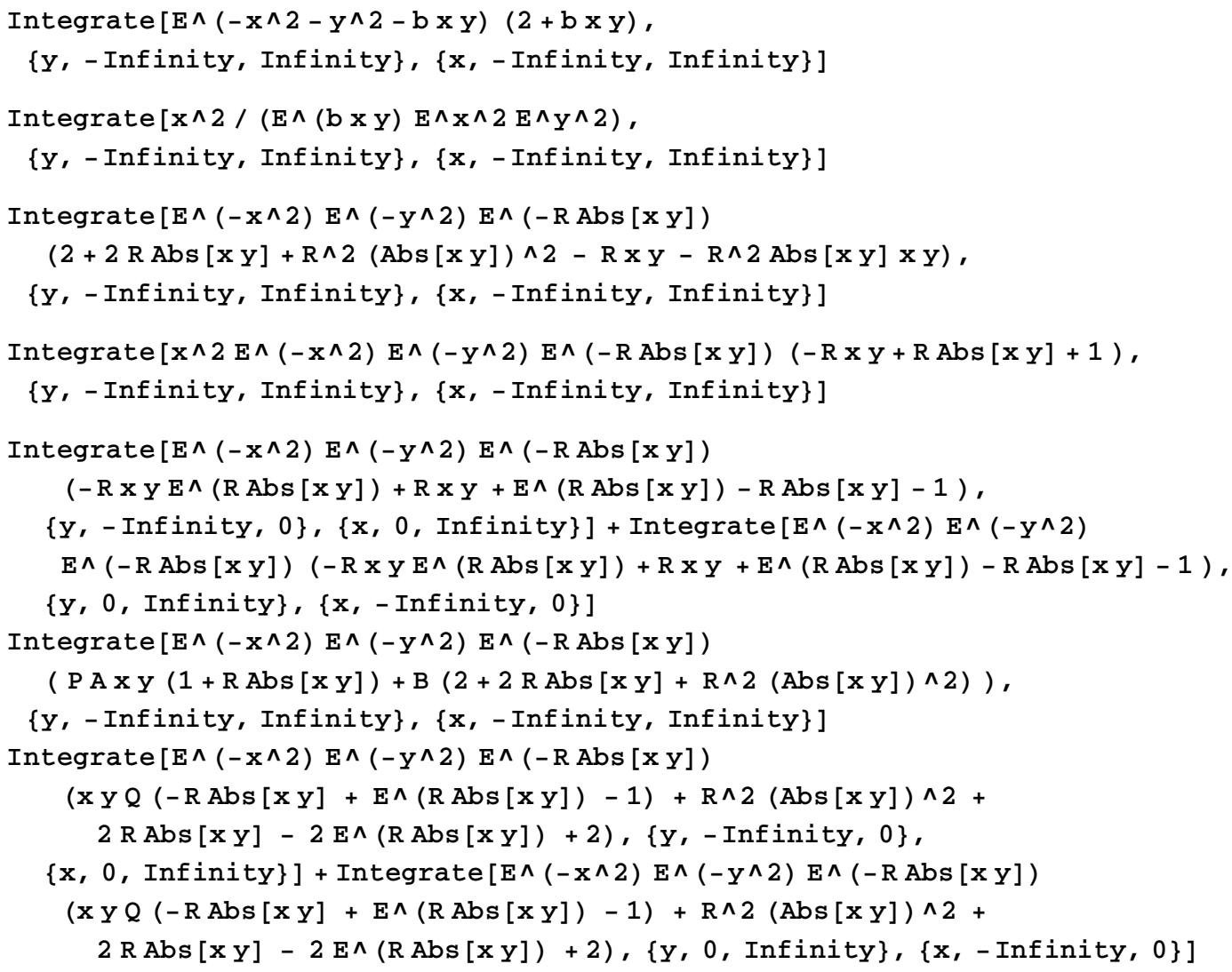




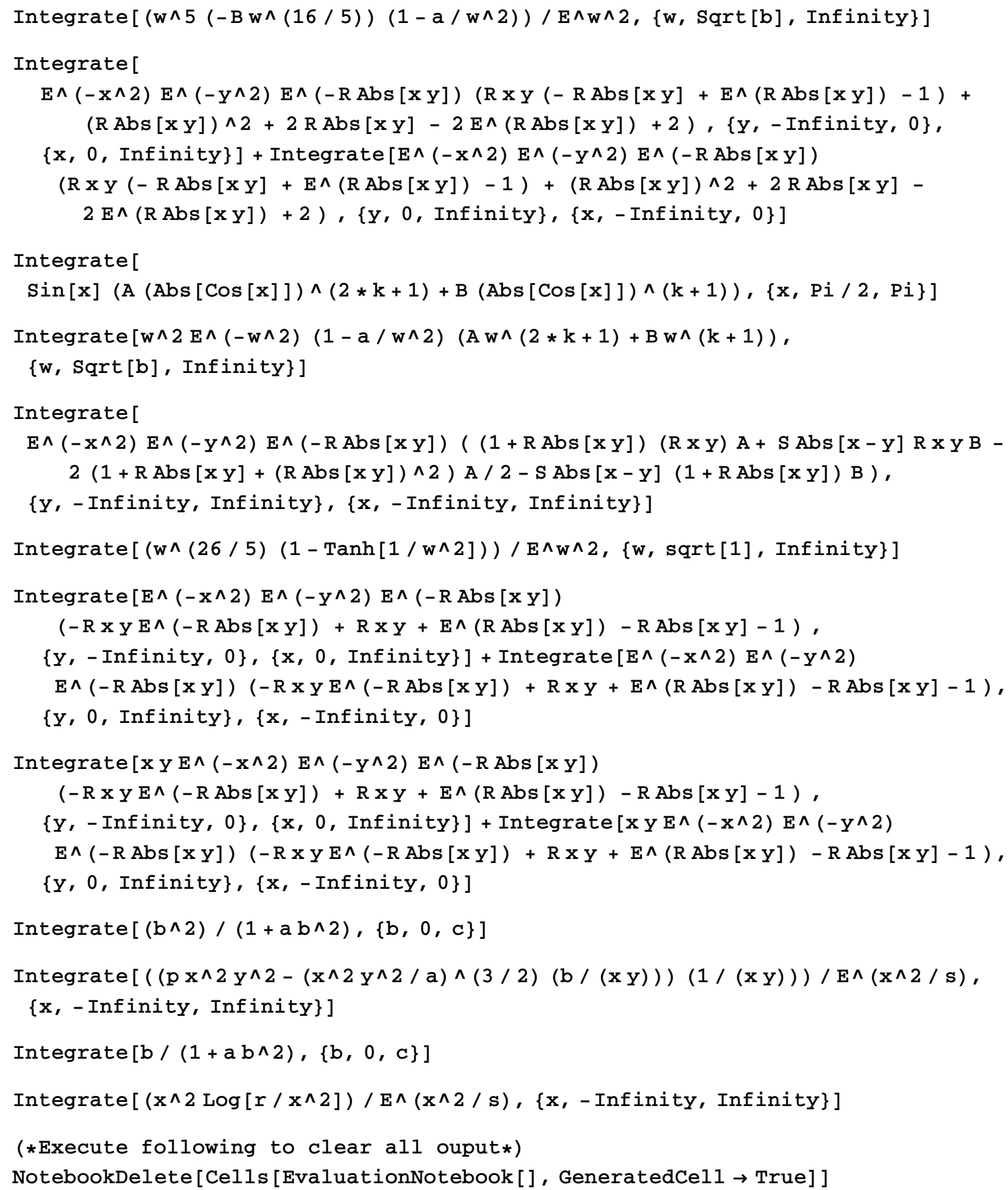





\section{Appendix 5: Highly nonlinear regions}
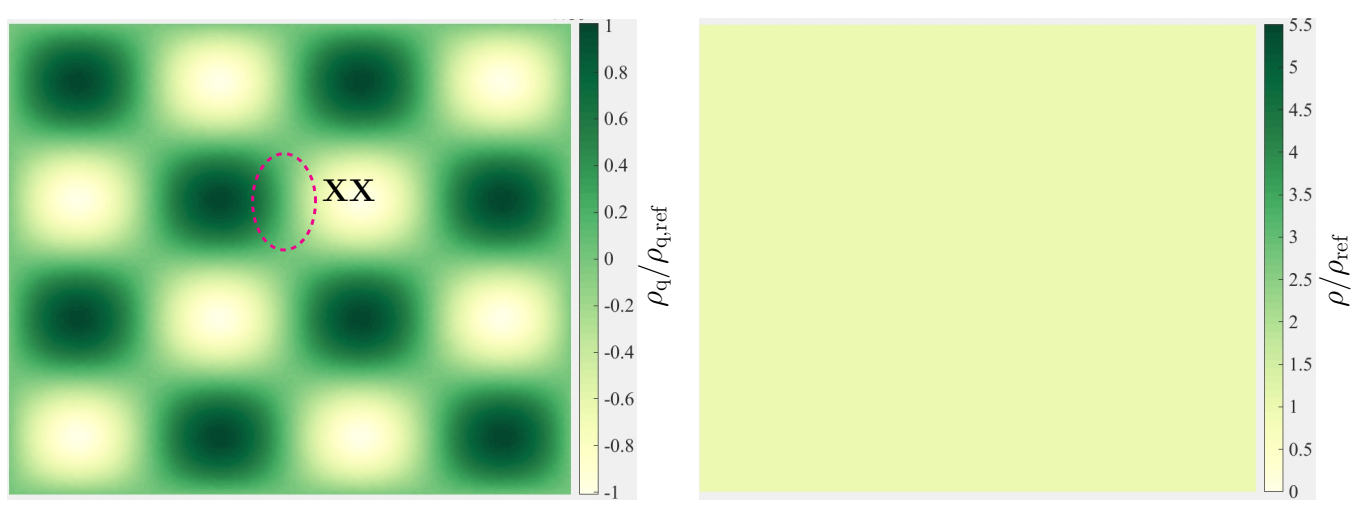

(a) $t / t_{0}=3.48 \times 10^{2}$
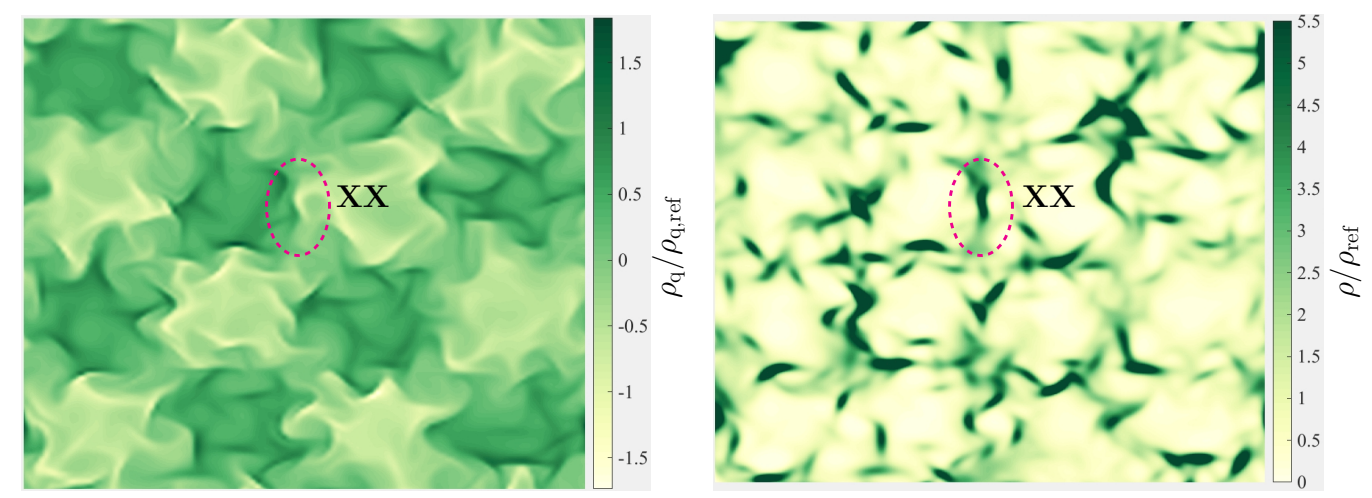

(b) $t / t_{0}=1.19 \times 10^{12}$

Figure A5.3: Typical time evolution of the hydrodynamic equations (6.26) introduced in chapter 6 . The left and right-hand side panels, respectively, show the macroscopic charge and mass density fields, normalized by some appropriate reference values. (a) The initial charge density (top left) is set to emphasize a possible scenario of charge separation, as schematically shown in Fig. 6.4. (b) It is shown that the regions (marked by XX) for which the terms linear in $\delta \tilde{\rho}_{\mathrm{q}}$ or $\delta \tilde{\varphi}$ in chapter 6 are expected to vanish, indeed develop high mass densities (bottom right, marked by XX). The results from the linear stability analysis presented in chapter 6 are thus valid away from these regions where a finite $\rho_{\mathrm{q} 0}$ approximation is reasonable. Credits: In this particular figure, the hydrodynamic code was developed by Dr. Mathias Hummel (Hummel et al. , 2016), while the electrostatic part was added by Chamkor Singh. 



\section{References}

Allen, Michael P, \& Tildesley, Dominic J. 2017. Computer simulation of liquids. Oxford university press.

Anderson, Robert, Björnsson, Sveinbjörn, Blanchard, Duncan C, Gathman, Stuart, Hughes, James, Jónasson, Sigurgeir, Moore, Charles B, Survilas, Henry J, \& Vonnegut, Bernard. 1965. Electricity in volcanic clouds. Science, 148(3674), 1179-1189.

Antony, S Joseph, Hoyle, William, \& Ding, Yulong. 2004. Granular materials: fundamentals and applications. Royal Society of Chemistry.

Aranson, Igor S, \& Tsimring, Lev S. 2006. Patterns and collective behavior in granular media: Theoretical concepts. Reviews of Modern Physics, 78(2), 641.

Bailey, Adrian G. 1998. The science and technology of electrostatic powder spraying, transport and coating. J. Electrostatics, 45(2), 85-120.

Ben-Avraham, Daniel, Ben-Naim, Eli, Lindenberg, Katja, \& Rosas, Alexandre. 2003. Self-similarity in random collision processes. Phys. Rev. E, 68(5), 050103.

Ben-Naim, Eli, \& Krapivsky, Paul L. 2000. Multiscaling in inelastic collisions. Phys. Rev. $E, \mathbf{6 1}(1), \mathrm{R} 5$.

Blum, J, Wurm, G, Kempf, S, Poppe, T, Klahr, H, Kozasa, T, Rott, M, Henning, Th, Dorschner, J, Schräpler, R, et al. . 2000. Growth and form of planetary seedlings: Results from a microgravity aggregation experiment. Phys. Rev. Lett., 85(12), 2426.

Blum, Jürgen. 2006. Dust agglomeration. Adv. Phys., 55(7-8), 881-947.

Blum, Jürgen, \& Wurm, Gerhard. 2008. The growth mechanisms of macroscopic bodies in protoplanetary disks. Annu. Rev. Astron. Astrophys., 46, 21-56.

Bodrova, Anna, Chechkin, Aleksei V, Cherstvy, Andrey G, \& Metzler, Ralf. 2015. Quantifying non-ergodic dynamics of force-free granular gases. Phys. Chem. Chem. Phys., 17(34), 21791-21798.

Bodrova, Anna S, Chechkin, Aleksei V, Cherstvy, Andrey G, Safdari, Hadiseh, Sokolov, Igor M, \& Metzler, Ralf. 2016. Underdamped scaled Brownian motion:(non-) existence of the overdamped limit in anomalous diffusion. Sci. Rep., 6, 30520.

Brey, J Javier, Ruiz-Montero, MJ, \& Cubero, D. 1999. Origin of density clustering in a freely evolving granular gas. Physical Review E, 60(3), 3150.

Brilliantov, Nikolai, Saluena, Clara, Schwager, Thomas, \& Pöschel, Thorsten. 2004. Transient structures in a granular gas. Physical Review Letters, 93(13), 134301.

Brilliantov, Nikolai, Krapivsky, P L, Bodrova, Anna, Spahn, Frank, Hayakawa, Hisao, Stadnichuk, Vladimir, \& Schmidt, Jürgen. 2015. Size distribution of particles in Saturn's rings from aggregation and fragmentation. Proc. Natl. Acad. Sci. USA, 112(31), 9536-9541. 
Brilliantov, Nikolai V, \& Pöschel, Thorsten. 2000a. Self-diffusion in granular gases. Phys. Rev. E, 61(2), 1716.

Brilliantov, Nikolai V, \& Pöschel, Thorsten. 2000b. Velocity distribution in granular gases of viscoelastic particles. Physical Review E, 61(5), 5573.

Brilliantov, Nikolai V, \& Pöschel, Thorsten. 2010. Kinetic theory of granular gases. Oxford University Press.

Brilliantov, Nikolai V, \& Spahn, Frank. 2006. Dust coagulation in equilibrium molecular gas. Math. Comput. Simulat., 72(2), 93-97.

Brilliantov, Nikolai V, Spahn, Frank, Hertzsch, Jan-Martin, \& Pöschel, Thorsten. 1996. Model for collisions in granular gases. Phys. Rev. E, 53(5), 5382.

Brilliantov, Nikolai V, Formella, Arno, \& Pöschel, Thorsten. 2018. Increasing temperature of cooling granular gases. Nature Communications, 9(1), 797.

Brito, R, \& Ernst, MH. 1998. Extension of Haff's cooling law in granular flows. EPL (Europhysics Letters), 43(5), 497.

Campbell, Charles S. 1990. Rapid granular flows. Annual Review of Fluid Mechanics, 22(1), 57-90.

Capelo, Holly L, Moláček, Jan, Lambrechts, Michiel, Lawson, John, Johansen, Anders, Blum, Jürgen, Bodenschatz, Eberhard, \& Xu, Haitao. 2019. Observation of aerodynamic instability in the flow of a particle stream in a dilute gas. Astronomy \& Astrophysics, 622, A151.

Castellanos, A. 2005. The relationship between attractive interparticle forces and bulk behaviour in dry and uncharged fine powders. Advances in Physics, 54(4), 263-376.

Cheng, L, \& Soo, SL. 1970. Charging of dust particles by impact. Journal of Applied Physics, 41(2), 585-591.

Crozier, W D. 1964. The electric field of a New Mexico dust devil. J. Geophys. Res., 69(24), 5427-5429.

Dammer, Stephan M, \& Wolf, Dietrich E. 2004. Self-focusing dynamics in monopolarly charged suspensions. Phys. Rev. Lett., 93(15), 150602.

Das, Prasenjit, Puri, Sanjay, \& Schwartz, Moshe. 2016. Clustering and velocity distributions in granular gases cooling by solid friction. Phys. Rev. E, 94(3), 032907.

Dash, JG, Mason, BL, \& Wettlaufer, JS. 2001. Theory of charge and mass transfer in ice-ice collisions. Journal of Geophysical Research: Atmospheres, 106(D17), 2039520402.

de Gennes, Pierre-Gilles. 1999. Granular matter: a tentative view. Reviews of Modern Physics, 71(2), S374.

Di Renzo, M, \& Urzay, J. 2018. Aerodynamic generation of electric fields in turbulence laden with charged inertial particles. Nature Communications, 9. 
Franz, RC, Nemzek, RJ, \& Winckler, JR. 1990. Television image of a large upward electrical discharge above a thunderstorm system. Science, 249(4964), 48.

Freier, G. D. 1960. The electric field of a large dust devil. J. Geophys. Res., 65(10), 3504-3504.

Friedl, Peter, \& Gilmour, Darren. 2009. Collective cell migration in morphogenesis, regeneration and cancer. Nature Reviews Molecular Cell Biology, 10(7), 445.

Gill, E W B. 1948. Frictional electrification of sand. Nature, 162, 568-569.

Glor, Martin. 1985. Hazards due to electrostatic charging of powders. J. Electrostatics, 16(2-3), 175-191.

Goldhirsch, ISAAC, \& Zanetti, G. 1993. Clustering instability in dissipative gases. Physical Review Letters, 70(11), 1619.

Gonzalez, S, Thornton, Anthony Richard, \& Luding, Stefan. 2014. Free cooling phasediagram of hard-spheres with short-and long-range interactions. The European Physical Journal Special Topics, 223(11), 2205-2225.

Grossman, EL, Zhou, Tong, \& Ben-Naim, E. 1997. Towards granular hydrodynamics in two dimensions. Phys. Rev. E, 55(4), 4200.

Gu, Zhaolin, Wei, Wei, Su, Junwei, \& Yu, Chuck Wah. 2013. The role of water content in triboelectric charging of wind-blown sand. Scientific Reports, 3, 1337.

Haeberle, Jan, Schella, André, Sperl, Matthias, Schröter, Matthias, \& Born, Philip. 2018. Double origin of stochastic granular tribocharging. Soft Matter.

Haff, PK. 1983. Grain flow as a fluid-mechanical phenomenon. Journal of Fluid Mechanics, 134, 401-430.

Harper, J S Méndez, McDonald, G D, Dufek, J, Malaska, M J, Burr, D M, Hayes, A G, McAdams, J, \& Wray, J J. 2017. Electrification of sand on Titan and its influence on sediment transport. Nature Geoscience, 10(4), 260.

Hertz, Heinrich. 1882. Über die Berührung fester elastischer Körper. Journal für die reine und angewandte Mathematik, $\mathbf{9 2}, 156$.

Horanyi, M, \& Goertz, CK. 1990. Coagulation of dust particles in a plasma. The Astrophysical Journal, 361, 155-161.

Hu, W, Xie, L, \& Zheng, X. 2012. Contact charging of silica glass particles in a single collision. Appl. Phys. Lett., 101(11), 114107.

$\mathrm{Hu}$, Wenwen, \& Xie, Li. 2016. Probability density functions of the net charge transfer of saltating particles in sand flux. Page 250001 of: AIP Conference Proceedings, vol. 1738. AIP Publishing.

Hummel, Mathias, Clewett, James PD, \& Mazza, Marco G. 2016. A universal scaling law for the evolution of granular gases. EPL (Europhysics Letters), 114(1), 10002. 
Ivlev, AV, Morfill, GE, \& Konopka, U. 2002. Coagulation of charged microparticles in neutral gas and charge-induced gel transitions. Phys. Rev. Lett., 89(19), 195502.

Jaeger, Heinrich M, Nagel, Sidney R, \& Behringer, Robert P. 1996. Granular solids, liquids, and gases. Reviews of Modern Physics, 68(4), 1259.

Johnson, Adam P., Cleaves, H. James, Dworkin, Jason P., Glavin, Daniel P., Lazcano, Antonio, \& Bada, Jeffrey L. 2008. The Miller Volcanic Spark Discharge Experiment. Science, 322(5900), 404-404.

Jullien, R. 1987. Aggregation phenomena and fractal aggregates. Contemporary Physics, 28(5), 477-493.

Jungmann, Felix, Steinpilz, Tobias, Teiser, Jens, \& Wurm, Gerhard. 2018. Sticking and restitution in collisions of charged sub-mm dielectric grains. Journal of Physics Communications, 2(9), 095009.

Kamra, A K. 1972. Physical sciences: Visual observation of electric sparks on gypsum dunes. Nature, 240(5377), 143-144.

Kempf, Sascha, Pfalzner, Susanne, \& Henning, Thomas K. 1999. N-particle-simulations of dust growth: I. growth driven by Brownian Motion. Icarus, 141(2), 388-398.

Kolehmainen, Jari, Ozel, Ali, Boyce, Christopher M, \& Sundaresan, Sankaran. 2016. A hybrid approach to computing electrostatic forces in fluidized beds of charged particles. AIChE Journal, 62(7), 2282-2295.

Kolehmainen, Jari, Ozel, Ali, Boyce, Christopher M, \& Sundaresan, Sankaran. 2017. Triboelectric charging of monodisperse particles in fluidized beds. AIChE Journal, 63(6), 1872-1891.

Kolehmainen, Jari, Ozel, Ali, Gu, Yile, Shinbrot, Troy, \& Sundaresan, Sankaran. 2018a. Effects of polarization on particle-laden flows. Physical Review Letters, 121(12), 124503.

Kolehmainen, Jari, Ozel, Ali, Gu, Yile, Shinbrot, Troy, \& Sundaresan, Sankaran. 2018b. Effects of Polarization on Particle-Laden Flows. Phys. Rev. Lett., 121(Sep), 124503.

Konopka, Ladislav, \& Kosek, Juraj. 2017. Discrete element modeling of electrostatic charging of polyethylene powder particles. Journal of Electrostatics, 87, 150-157.

Konopka, Uwe, Mokler, Felicitas, Ivlev, Alexei V, Kretschmer, Michael, Morfill, Gregor E, Thomas, Hubertus M, Rothermel, Hermann, Fortov, Vladimir E, Lipaev, Andrey M, Molotkov, Vladimir I, et al. . 2005. Charge-induced gelation of microparticles. New Journal of Physics, 7(1), 227.

Kuwabara, Goro, \& Kono, Kimitoshi. 1987. Restitution coefficient in a collision between two spheres. Jpn. J. Appl. Phys., 26(8R), 1230.

Lacks, Daniel J, \& Sankaran, R Mohan. 2011. Contact electrification of insulating materials. J. Phys. D: Appl. Phys., 44(45), 453001. 
Landau, LD, \& Lifshitz, EM. 2000. Mechanics: Volume 1 (Course of Theoretical Physics Series).

Lebovka, Nikolai I. 2012. Aggregation of charged colloidal particles. Pages 57-96 of: Polyelectrolyte Complexes in the Dispersed and Solid State I. Springer.

Lee, Victor, Waitukaitis, Scott R, Miskin, Marc Z, \& Jaeger, Heinrich M. 2015. Direct observation of particle interactions and clustering in charged granular streams. Nature Physics, 11(9), 733-737.

Lee, Victor, James, Nicole M, Waitukaitis, Scott R, \& Jaeger, Heinrich M. 2018. Collisional charging of individual submillimeter particles: Using ultrasonic levitation to initiate and track charge transfer. Physical Review Materials, 2(3), 035602.

Leyvraz, François. 2003. Scaling theory and exactly solved models in the kinetics of irreversible aggregation. Phys. Rep., 383(2), 95-212.

Liu, Peiyuan, \& Hrenya, Christine M. 2018. Cluster-Induced Deagglomeration in Dilute Gravity-Driven Gas-Solid Flows of Cohesive Grains. Phys. Rev. Lett., 121(Dec), 238001.

Lowell, J. 1991. Mechanisms of contact charging and charge accumulation: experiments on soda glass. Journal of Physics D: Applied Physics, 24(3), 375.

Lübeck, Sven. 2004. Universal scaling behavior of non-equilibrium phase transitions. Int. J. Mod. Phys. B, 18(31n32), 3977-4118.

Luding, STEFAN, \& Herrmann, HANS J. 1999. Cluster-growth in freely cooling granular media. Chaos, 9(3), 673-681.

Ma, Qianyu, Matthews, Lorin S, Land, Victor, \& Hyde, Truell W. 2013. Charging of aggregate grains in astrophysical environments. The Astrophysical Journal, 763(2), 77.

Mandelbrot, Benoit. 1977. Fractals. Freeman San Francisco.

Marshall, JR, Sauke, TB, \& Cuzzi, JN. 2005. Microgravity studies of aggregation in particulate clouds. Geophysical Research Letters, 32(11).

Matias, AFV, Shinbrot, Troy, \& Araújo, NAM. 2018. Mechanical equilibrium of aggregates of dielectric spheres. Physical Review E, 98(6), 062903.

Matthews, Lorin S, Shotorban, Babak, \& Hyde, Truell W. 2018. Discrete stochastic charging of aggregate grains. Physical Review E, 97(5), 053207.

Matthews, Lorin Swint, \& Hyde, Truell W. 2004. Effects of the charge-dipole interaction on the coagulation of fractal aggregates. IEEE Transactions on Plasma Science, 32(2), $586-593$.

Matthews, LS, \& Hyde, TW. 2009. Effect of dipole-dipole charge interactions on dust coagulation. New Journal of Physics, 11(6), 063030.

McNamara, Sean. 1993. Hydrodynamic modes of a uniform granular medium. Physics of Fluids A: Fluid Dynamics, 5(12), 3056-3070. 
Mehrotra, Amit, Muzzio, Fernando J., \& Shinbrot, Troy. 2007. Spontaneous Separation of Charged Grains. Phys. Rev. Lett., 99(Jul), 058001.

Melnik, Olga, \& Parrot, Michel. 1998. Electrostatic discharge in Martian dust storms. $J$. Geophys. Res., 103(A12), 29107-29117.

Mikkelsen, René, van der Meer, Devaraj, van der Weele, Ko, \& Lohse, Detlef. 2002. Competitive clustering in a bidisperse granular gas. Phys. Rev. Lett., 89(21), 214301.

Miller, S, \& Luding, S. 2004. Cluster growth in two-and three-dimensional granular gases. Phys. Rev. E, 69(3), 031305.

Moore, Arthur Dearth. 1972. Electrostatics. Scientific American, 226(3), 46-59.

Most, Glenn W. 2006. Hesiod: Theogony, Works and Days, Testimonia. Vol. 1. Harvard University Press.

Müller, Micha-Klaus. 2008. Long-range interactions in dilute granular systems. University of Twente.

Müller, Micha-Klaus, \& Luding, Stefan. 2011. Homogeneous cooling with repulsive and attractive long-range potentials. Mathematical Modelling of Natural Phenomena, 6(4), $118-150$.

Murphy, Eric, \& Subramaniam, Shankar. 2015. Freely cooling granular gases with shortranged attractive potentials. Phys. Fluids, 27(4), 043301.

Nie, Xiaobo, Ben-Naim, Eli, \& Chen, Shiyi. 2002. Dynamics of freely cooling granular gases. Phys. Rev. Lett., 89(20), 204301.

Nifuku, M., Ishikawa, T., \& Sasaki, T. 1989. Static electrification phenomena in pneumatic transportation of coal. J. Electrostatics, 23(Supplement C), 45 - 54.

Nordsiek, Freja, \& Lathrop, Daniel P. 2015. Collective phenomena in granular and atmospheric electrification. arXiv preprint arXiv:1509.04214.

Pähtz, Thomas, Herrmann, Hans J, \& Shinbrot, Troy. 2010. Why do particle clouds generate electric charges? Nature Physics, 6(5), 364-368.

Pathak, Sudhir N, Jabeen, Zahera, Das, Dibyendu, \& Rajesh, R. 2014. Energy decay in three-dimensional freely cooling granular gas. Phys. Rev. Lett., 112(3), 038001.

Paul, Subhajit, \& Das, Subir K. 2017. Ballistic aggregation in systems of inelastic particles: Cluster growth, structure, and aging. Phys. Rev. E, 96(Jul), 012105.

Pingali, Kalyana C., Hammond, Stephen V., Muzzio, Fernando J., \& Shinbrot, Troy. 2009. Use of a static eliminator to improve powder flow. Int. J. Pharm., 369(1), 2 - 4.

Pitaevskii, LP, \& Lifshitz, EM. 2012. Physical kinetics. Vol. 10. Butterworth-Heinemann.

Poppe, T, \& Schräpler, R. 2005. Further experiments on collisional tribocharging of cosmic grains. Astron. Astrophys., 438(1), 1-9. 
Poppe, Torsten, Blum, Jürgen, \& Henning, Thomas. 2000a. Analogous experiments on the stickiness of micron-sized preplanetary dust. Astrophys. J., 533(1), 454.

Poppe, Torsten, Blum, Jürgen, \& Henning, Thomas. 2000b. Experiments on collisional grain charging of micron-sized preplanetary dust. Astrophys. J., 533(1), 472.

Pöschel, Thorsten, \& Schwager, Thomas. 2005. Computational granular dynamics: models and algorithms. Springer Science \& Business Media.

Pöschel, Thorsten, Brilliantov, Nikolai V, \& Schwager, Thomas. 2003. Long-time behavior of granular gases with impact-velocity dependent coefficient of restitution. Physica A, 325(1), 274-283.

Pu, Yu, Mazumder, Malay, \& Cooney, Charles. 2009. Effects of Electrostatic Charging on Pharmaceutical Powder Blending Homogeneity. J. Pharm. Sci., 98(7), 2412 - 2421.

Ramírez, Rosa, Pöschel, Thorsten, Brilliantov, Nikolai V, \& Schwager, Thomas. 1999. Coefficient of restitution of colliding viscoelastic spheres. Phys. Rev. E, 60(4), 4465.

Saunders, Clive. 2008. Charge separation mechanisms in clouds. Pages 335-353 of: Planetary Atmospheric Electricity. Springer.

Scheffler, T, \& Wolf, D E. 2002. Collision rates in charged granular gases. Granular Matter, 4(3), 103-113.

Schella, André, Weis, Simon, \& Schröter, Matthias. 2017. Charging changes contact composition in binary sphere packings. Physical Review E, 95(6), 062903.

Schwager, Thomas, \& Pöschel, Thorsten. 1998. Coefficient of normal restitution of viscous particles and cooling rate of granular gases. Physical review E, 57(1), 650.

Schwager, Thomas, Wolf, Dietrich E, \& Pöschel, Thorsten. 2008. Fractal substructure of a nanopowder. Physical Review Letters, 100(21), 218002.

Shinbrot, Troy, Rutala, Matthew, \& Herrmann, Hans. 2017. Surface contact charging. Physical Review E, 96(3), 032912.

Shinbrot, Troy, Ferdowsi, Behrooz, Sundaresan, Sankaran, \& Araujo, Nuno A. M. 2018. Multiple timescale contact charging. Phys. Rev. Materials, 2(Dec), 125003.

Simpson, George, \& Scrase, F J. 1937. The distribution of electricity in thunderclouds. Proc. Roy. Soc. London A, 161, 309-352.

Singh, Chamkor, \& Mazza, Marco G. 2018. Early-stage aggregation in three-dimensional charged granular gas. Physical Review E, 97(2), 022904.

Siu, Theo, Pittman, Will, Cotton, Jake, \& Shinbrot, Troy. 2015. Nonlinear granular electrostatics. Granular Matter, 17(2), 165-175.

Smirnov, Boris Mikhaullovich. 1990. The properties of fractal clusters. Physics Reports, 188(1), 1-78. 
Spahn, Frank, \& Seiß, Martin. 2015. Granular matter: Charges dropped. Nature Physics, 11(9), 709.

Stauffer, Dietrich. 1979. Scaling theory of percolation clusters. Phys. Rep., 54(1), 1-74.

Stauffer, Dietrich, \& Aharony, Amnon. 2003. Introduction to percolation theory. Taylor \& Francis.

Stow, C D. 1969. Dust and sand storm electrification. Weather, 24(4), 134-144.

Takada, Satoshi, Saitoh, Kuniyasu, \& Hayakawa, Hisao. 2016. Kinetic theory for dilute cohesive granular gases with a square well potential. Phys. Rev. E, 94(1), 012906.

Takada, Satoshi, Serero, Dan, \& Pöschel, Thorsten. 2017. Homogeneous cooling state of dilute granular gases of charged particles. Phys. Fluids, 29(8), 083303.

Ulrich, Stephan, Aspelmeier, Timo, Roeller, Klaus, Fingerle, Axel, Herminghaus, Stephan, \& Zippelius, Annette. 2009. Cooling and aggregation in wet granulates. Physical Review Letters, 102(14), 148002.

van der Weele, Ko, van der Meer, Devaraj, Versluis, Michel, \& Lohse, Detlef. 2001. Hysteretic clustering in granular gas. EPL (Europhysics Letters), 53(3), 328.

Volpe, Giorgio, \& Volpe, Giovanni. 2013. Simulation of a Brownian particle in an optical trap. American Journal of Physics, 81(3), 224-230.

Wesson, Paul S. 1973. Accretion and electrostatic interaction of interstellar dust grains; Interstellar grit. Astrophys. Space Sci., 23(1), 227-255.

Wolf, Dietrich E, Pöschel, Thorsten, Schwager, Thomas, Weuster, Alexander, \& Brendel, Lothar. 2009. Fractal substructures due to fragmentation and reagglomeration. Pages 859-862 of: AIP Conference Proceedings, vol. 1145. AIP.

Yan, Jing, Han, Ming, Zhang, Jie, Xu, Cong, Luijten, Erik, \& Granick, Steve. 2016. Reconfiguring active particles by electrostatic imbalance. Nature Materials, 15(10), 1095.

Yoshimatsu, R, Araújo, N A M, Wurm, G, Herrmann, H J, \& Shinbrot, T. 2017. Selfcharging of identical grains in the absence of an external field. Sci. Rep., 7, 39996.

Yoshimatsu, Ryuta, Araújo, Nuno AM, Shinbrot, Troy, \& Herrmann, Hans Jürgen. 2016. Field driven charging dynamics of a fluidized granular bed. Soft Matter, 12(29), 62616267.

Zhang, Yanzhen, Pähtz, Thomas, Liu, Yonghong, Wang, Xiaolong, Zhang, Rui, Shen, Yang, Ji, Renjie, \& Cai, Baoping. 2015. Electric field and humidity trigger contact electrification. Physical Review X, 5(1), 011002.

Zheng, XiaoJing, Zhang, Rui, \& Huang, HaoJie. 2014. Theoretical modeling of relative humidity on contact electrification of sand particles. Scientific Reports, 4, 4399.

Zsom, Andras, Ormel, CW, Güttler, C, Blum, J, \& Dullemond, CP. 2010. The outcome of protoplanetary dust growth: pebbles, boulders, or planetesimals?-II. Introducing the bouncing barrier. Astronomy \& Astrophysics, 513, A57. 


\section{Curriculum Vitae}

\section{Current address}

Immanuel-Kant Str. 32

37083, Göttingen

Germany

\section{Education}

2016-2019: Doctoral student, IMPRS-PBCS, University of Göttingen

2013-2016: MS (by Research), Thermal Science and Engineering, IIT Kharagpur, India 2010-2013: Bachelor of Technology, Mechanical Engineering, LPU Punjab, India 2007-2010: Diploma, Mechanical Engineering, Government Polytechnic Nilokheri, India

\section{Publications from the thesis}

C. Singh and M.G. Mazza, Electrification in granular gases leads to constrained fractal growth, Scientific Reports 9: 9049 (2019).

C. Singh and M.G. Mazza, Early-stage aggregation in three-dimensional charged granular gas, Physical Review E 97: 022904 (2018).

\section{Invited talk from the thesis}

Particle aggregation in 3-D charged granular gas: simulations and mean-field study, Gordon Research Seminar - Granular Matter (Frontiers of Granular Physics Session), Easton, Massachusetts, USA, July 2018.

\section{Tutorship}

Statistical mechanics and thermodynamics, winter-semester 2017/18.

Introduction to data science (one week course), winter-semester 2017/18.

Machine learning and pattern recognition, summer-semester 2018.

Guided a Ludwig-Prandtl-Internship at MPIDS, June-September 2018.

\section{Personal information}

Date of birth: 22.01.1991 

Thanks to the developers of LT $_{\mathrm{E}} \mathrm{X}$, Linux, i3wm, MATLAB, MATHEMATICA, NVIDIACUDA, and Overleaf. Also special thanks to the IT personnel of the MPIDS.

I confirm that I have written this thesis independently and without the help of any other source or aid than quoted.

Chamkor Singh, Göttingen, July 2019. 